

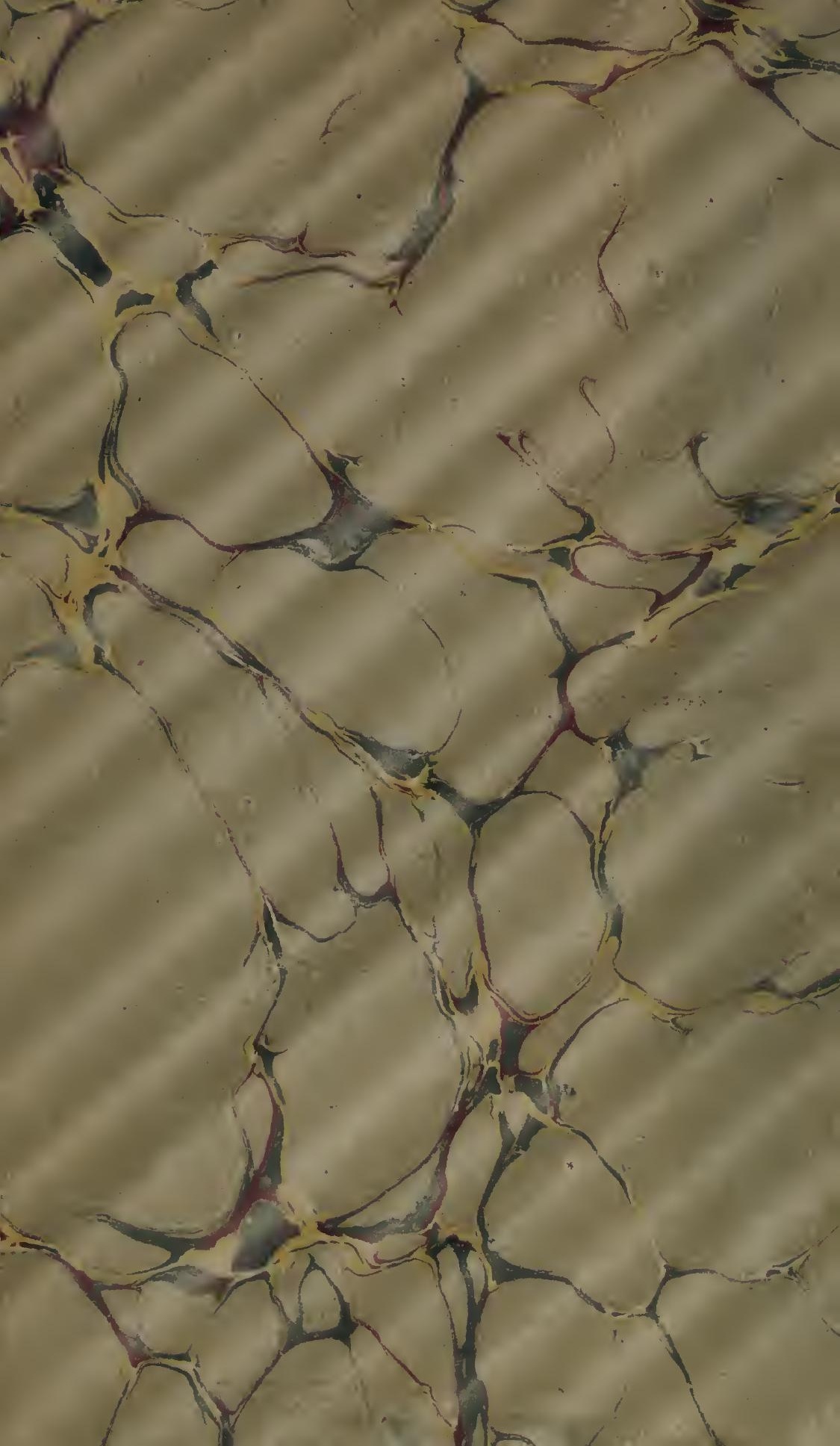




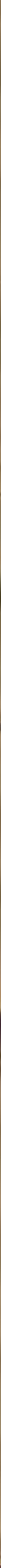


n 
$-$ 
ÉTUDE S

$S T R$

\section{L'A MÉNAGEMENT}

DES FORETS 


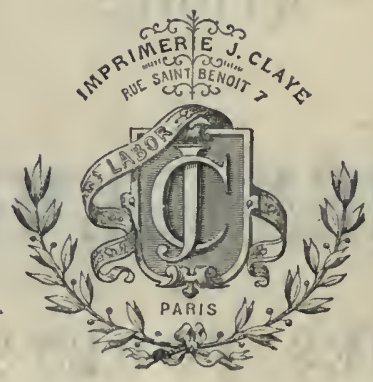




\section{É T UDES}

$\mathbf{S} \mathbf{U} \mathbf{R}$

\section{L'AMENAGEMENT \\ DES FORETTS}

PA

L. T A S S Y

COXSBRVATBUR DBB FORATS,

ANCIEY PROFBSSER A LIKSTITUT AOEONOMLQUE DE VERSAILLES

DEUXIEME EDITION, REVUE ET AUGMENTÉE
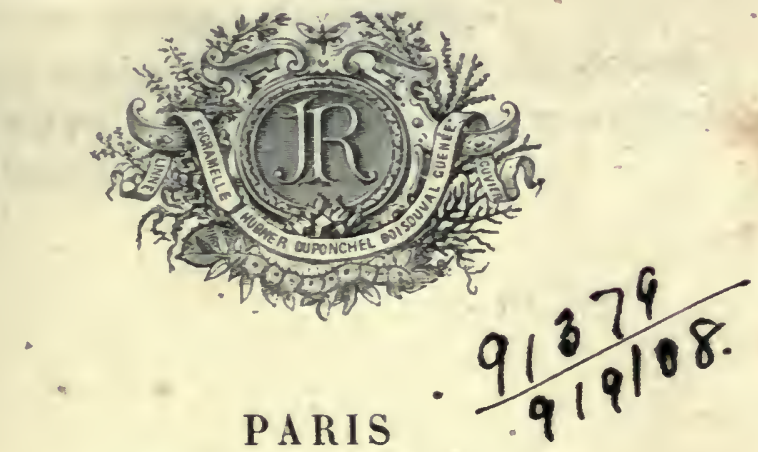

J. ROTHSGHILD, ÉDITEUR

LIBRAIRE DE LA SOCIÉTÉ BOTANIQUE DE FRANCE

13, RUE DES SAINTS-PÉRES, 13

\section{2}

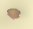

Droits résertés.

(

\section{LIBRARY}




$$
\begin{aligned}
& \text { SD } \\
& 431 \\
& T 37 \\
& 1872
\end{aligned}
$$

$$
801_{p}^{0} p
$$




\section{A II. ADOLPHE LORENTZ}

\section{Hov CHER AMI,}

J'ai dédie la première édition de ces Études à notre cher. et regretté maitre, M. Parade. Je te dédie celle-ci; car parmi tous nos camarades, je n'en vois aucun qui soil plus que toi lobservateur fidèle el dèvoué des préceptes qu'il nous a laissés.

Tu as collaborè d"ailleurs aux améliorations que je crois avoir apportées à mon premier travail, et je suis heureux de pouvoir t'en exprimer ma reconnaissance.

Enfin, tu portes dignement un nom que tous les forestiers iènèrent et qui, placè en têle de cet ouvrage, ne peut que lui porter bonheur.

\section{LOVIS TASSY.}




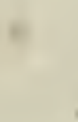
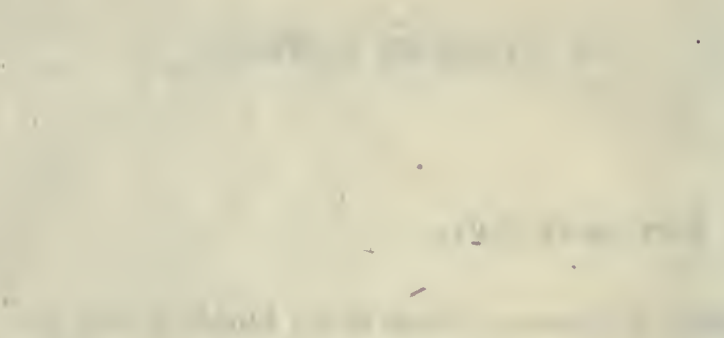

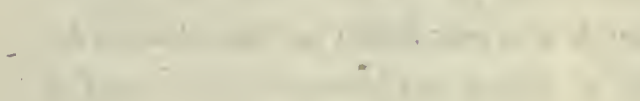
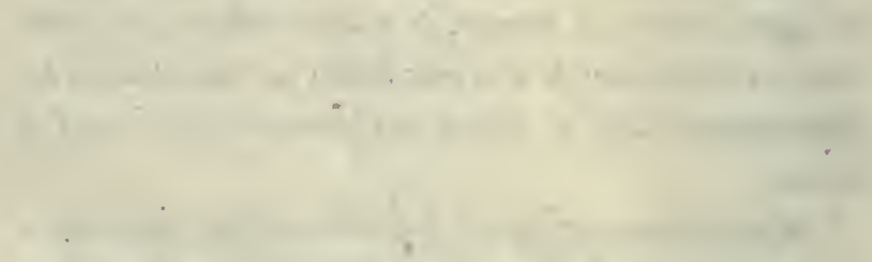

..$+\ldots$

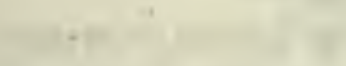

*

-
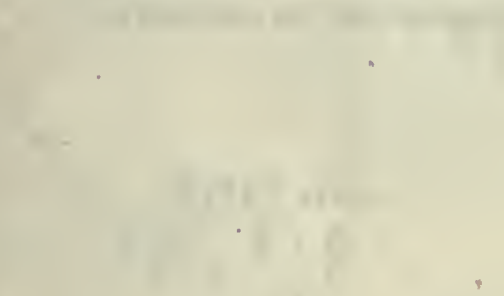

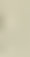

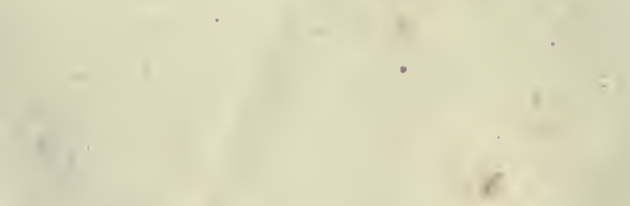

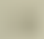

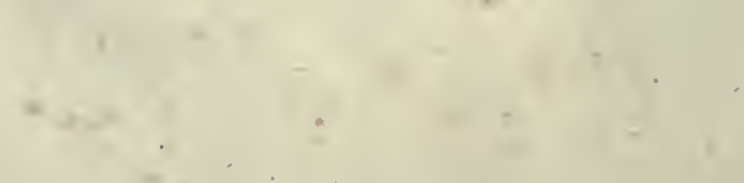




\section{PRÉFACE.}

Des calamités sans exemple ont frappé notre pays; il ne m'appartient pas d'en rechercher ici les causes; je dirai seulement qu'on trouverait peut-être la principale de ces causes, celle qui a engendré toutes les autres, dans ce dévergondage des appétits matériels, que je déplorais déjà dans la préface de. la première édition de ces Études comme étant incompatible avec la conservation des forêts, et qu'on remarque nonseulement chez les hommes illettrés, mais aussi, il faut bien l'avouer, chez ceux qui, par leurs richesses, leur éducation et leurrs lumières, sont appélés à diriger les autres. "Plus d'États ont péri parce qu'on a violé les moeurs que parce qu'on a violé les lois ${ }^{1}$ ", et il ne faut pas beau-

1. Montesquieu, Décadence des Romains. 
coup connaître l'histoire pour savoir que de tout temps la décadence des nations a été déterminée par la corruption des hautes classes. On reproche au prolétariat ses convoitises matérielles! Et celles des gens qui possèdent, sontelles plus excusables et moins dangereuses? Hélas! le matérialisme règne dans les palais comme dans les chaumières; il est chez l'ignorant, il est chez le savant; le pauvre s'y livre sans le savoir, le riche en a fait un dogme; il est partout, résultat chez les. uns d'un extrême sensualisme, résultat chez les autres de l'orgueil et de la puissance exagérée qu'ils attribuent à leur intelligence.

Quoi qu'il en soit, quand un grand peuple comme le nôtre, si grand encore-malgré ses revers, a été profondément atteint dans sa fortune, il n'a qu'un moyen de se relever : supprimer toutes les dépenses de luxe et augmenter les dépenses utiles. Si, en même temps qu'il supprimerait les premières, il réduisait aussi les dernières, il consommerait infailliblement sa ruine.

Mais de toutes les dépenses, les plus utiles sont certainement celles qui ont pour objet l'amélioration du sol. 11 y a donc lieu d'espérer 
que la dotation accordée à l'arlministration des forêts, pour les travaux dont elle est chargée, au lieu d'avoir à souffrir des cruels événements que nous renons de traverser, va, au contraire, sortir des limites trop étroites, dans lesquelles on l'a enfermée jusqu'à présent, et que les louables efforts faits par cette administration, pour donner plus d'intensité à la production du sol forestier, seront encouragés davantage. Parmi ces efforts, les plus remarquables, quoique les moins remarqués, sont ceux qui se rapportent à la conversion des taillis en futaies pleines. Ils honoreront éternellement les forestiers de notre époque et, avant tous, l'illustre fondateur de l'école forestiêre, M. Lorentz, car c'est à lui sans contredit que revient l'initiative de cette grande opération. Mais il ne suffit pas de l'avoir entreprise; il faut maintenant se résigner franchement à des privations temporaires si on veut qu'elle soit efficace, et j'ai peur que, sous ce rapport, l'administration supérieure ne soit pas disposée à faire preuve de tout le désintéressement nécessaire. Il y a là un danger grave que reconnaîtront, je l'espère, ceux qui se donneront la peine de lire et de méditer la troisième étude de mon livre : si, de tous les travaux d'aména- 
gement, celui qui consiste dans la conversion d'un taillis en futaie est le plus avantageux pour l'avenir, quand il est fait dans de certaines conditions, il peut devenir, par contre, un des plus fâcheux par ses conséquences, lorsqu'on lui marchande les sacrifices sur lesquels reposé son succès.

L'aménagement, quand il a pour objet de convertir un taillis en futaie, ne saurait s'accomplir sans entraîner une diminution temporaire, presque immédiate, dans la production de la forêt; et plus cette diminution est forte, plus rapide est la transformation. Tout cela se comprend si l'on considère qu'au fond et en définitive, la conversion d'un taillis en futaie con- . siste à transformer un massif composé d'un petit nombre de coupes (30 à 40) en un massif composé d'un grand nombre de coupes (100 et au delà). Cependant l'administration supérieure, tout en reconnaissant l'utilité de ces sortẹs d'aménagements, hésite à les faire sanctionner, dès qu'ils accusent une diminution de revenu, et il est facile de deviner que si cette préoccu-pation de la réduction temporaire des produits empêche les agents forestiers de réaliser dans la gestion des forêts domaniales toutes les amélio- 
rations désirables, elle arrive à paralyser leur action et leur bonne volonté, relativement aux forêts qui appartiennent aux communes; car les communes sont naturellement bien moins disposées que l'État à sacrifier le présent à l'avenir.

On constate donc encore, au sujet des conversions, la même diversité de vues et de tendances que l'on a signalée tant de fois déjà, et notamment à propos de l'aliéuation des forêts, entre l'administration forestière et le ministre dont elle relève, diversité de vues et de tendances qui est d'ailleurs facile à comprendre : la miṣsion dont le ministre des finances est clargé, et qui vise surtout à maintenir entre les dépenses et les recettes de l'État un équilibre sans lequel la marche des services publics serait infailliblement compromise, est certes très-respectable; malheureusement, elle est en contradiction avec les travaux dont l'effet utile n'est pas susceptible de se produire immédiatement, et elle donne á l'esprit une circonspection et des habitudes de parcimonie qui sont tout à fait l'opposé de la hardiesse et de la libéralité dont il faudrait être pourvu, pour aborder et résoudre d'une manière satisfaisante les questions relatives au 
développement indéfini des richesses morales et matérielles de la nation.

Les hommes qui, comme les forestiers, sont habitués à préparer des fruits dont ils ne jouiront pas; les hommes qui aiment à planter pour leurs petits neveux, sont naturellement enclins à grever le présent au profit de l'avenir. Que l'on modère ce penchant, je le comprends; mais il ne faut pas l'annihiler, cár après tout il est l'honneur des individus et le devoir des peuples. Le ministre des finances le supprimerait pourtant complétement si cela ne dépendait que de lui, et pour résumer la situation en deux mots : je dirai que le ministre des finances songe aux générations futures pour leur demander de l'argent, tandis que les forestiers au contraire cherchent les moyens de leur en procurer. Comment le ministère des finances et l'administration forestière pourraient-ils donc s'accorder? ils ne s'accorderont jamais et on leur rendrait grand service à tous les deux en prononçant leur séparation. Tous les hommes compétents insistent sur l'utilité, sur l'urgence de cette mesure. Pourquoi leur voix n'est-elle pas écoutée? Peut-être regarde-t-on le service forestier comme un détail de peu d'importance, au 
milieu des questions politiques et administratives qui agitent aujourd'hui les esprits. On se trompe si l'on a cette pensée : une administration qui gère un domaine auquel, suivant l'expression d'un grand ministre, "les sociétés demandent des éléments de protection, de sûreté et de gloire ", ne saurait être un détail de peu d'importance, et la réforme que l'on sollicite pour l'administration forestière, n'est point une de ces améliorations secondaires qui peuvent être ajournées sans inconvénient. Elle se lie évidemment à des intérêts d'un ordre trèsélevé, et il serait d'autant plus opportun de la réaliser que nos forêts, dont l'étendue a été diminuée d'au moins trois cent cinquante mille hectares par la dernière guerre, sont menacées maintenant par les projets de décentralisation administrative qui font tant de bruit depuis quelque temps, qui ont reçu un commencement d'exécution, et qui ne peuvent que favoriser la tendance de l'individu à s'approprier la chose publique.

Cette tendance est d'ailleurs le signe distinctif de toutes les civilisations avancées. A l'origine des sociétés, l'homme ne possède d'autres moyens d'action que ceux qu'il tient de ses 
facultés natives. Ces facultés natives font toute sa force, tout son pouvoir, tout son capital; il vit au jour le jour, n'économise pas et ne jouit exclusivement que du fruit de son travail. En dehors de lui et des objets qui servent à sa consommation, rien ne lui appartient en propre; tout est commun et reste commun à la peuplade ou à la tribu, tant que les choses restent ellesmêmes telles que la nature les a faites. Mais, dès que l'homme a mis son empreinte sur un objet; dès qu'il y a ajouté une valeur productive si minime qu'elle soit, l'indivision, la comniunauté des biens commence à lui peser, et il cherche dans l'appropriation un moyen d'augmenter sa puissance personnelle, en s'assimilant, en s'incorporant en quelque sorte des forces permanentes étrangères à son essence. Alors les sociétés progressent rapidement. Cependant, elles finiråient tôt ou tard par se désagréger si , à côté de cette tendance dè plus en plus' forte de l'individu à s'accroître aux dépens de la communauté, il ne s'établissait pas un pouvoir central, in pouvoir collectif dominant tous les autres, et assez puissant pour soustraire au morcellement les biens qui, étant utiles, nécessaires à tous, ne sauraient être 
abandonnés aux hasards de la destinée individuelle. C'est là ce qui justifie, ce qui légitime ce qu'on appelle la centralisation. Je n'ai point à expliquer ici comment peut se constituer ce pouvoir central et supérieur au milieu des personnalités qui sembleraient en être la négation. Je constate seulement qu'il est indispensable et qu'il ne saurait être anéanti, sans que la société fût transformée aussitôt en une agglomération d'individus qui, dépourvus de toute solidarité, de tout lien, seraient exposés à se disperser à tout instant: Or, parmi les biens communs qui forment comme le ciment du corps social, il n'en est pas de plus précieux que les forêts; on ne doit rien négliger de ce qui peut contribuer à les sauvegarder, et voilả pourquoi il serait nécessaire, d'abord, de les affranchir de l'autorité fiscale, attendu qu'au lieu de les défendre contre les convoitises de l'individu, elle est plutôt disposée à les leur livrer.

Je pense que pour les forêts domaniales, la suppression de cette autorité fiscale qui les a exclusivement gouvernées jusqu'à présent, suffirait à garantir leur conservation. Quant aux forêts communales', il leur faudrait une autre 
sauvegarde et je ne la vois que dans les mesures qui donneraient plus d'efficacité à la tutelle exercée par l'État sur ces forêts. Si, comme tant de gens le désirent, les forêts communales étạient remises à la libre disposition des conseils municipaux, c'en serait fait d'elles: comment voudrait-on en effet que les conseils municipaux pussent les protéger longtemps contre l'individualisme, puisque le gouvernement central, avec toute l'autorité dont il est investi, n'y réussit pas toujours?

J'émets là des idées qui sont contraires à ce mouvement vers la décentralisation dont je parlais tantôt; mais ce mouvement s'est déjà traduit par une loi; il constitue un très-sérieux danger pour la conservation du peu de forêts qui nous restent; je ne saurais me dispenser de m'y arrêter un moment.

Quel est le but que poursuivent ceux qui ont mis en avant ce mot de décentralisation? ils veulent, disent-ils, reconstituer l'autonomie des communes et celle des départements. Sur ce point les hommes des partis les plus opposés se donnent la main. L'autonomie est la panacée qui remédiera à tous les maux de l'humanité. Examinons ce que vaut ce grand mot-là : 
On entend par autonomie le droit de se gouverner par ses propres lois. Ėtre autonome, c'est se faire sa règle à soi-même; c'est être indépendant, vivre de sa propre intelligence, de sa propre force, sans secours étranger. Mais, s'il en est ainsi, il est évident que l'autonomie de l'individu', celle de la commune, celle du département et celle de l'État ne sauraient coexister. Formez-en une et les autrés disparaissent; d'où il suit qu'aucun des éléments qui composent la société, ne saurait prétendre à une indépendance absolue, pour peu qu'il reconnaisse l'utilité de conserver les autres : tonte la question est de savoir dans quelle proportion il convient que l'autorité se partage entre eux.

Remarquon's d'ailleurs que ces différents pouvoirs superposés se sont tous constitués aux dépens de la liberté individuelle; qu'ils n'ont pas de vertu propre; qu'ils n'existent que pour le bien de chacun, et qu'ils deviennent usurpateurs, dès qu'ils retiennent dans leur ensemble plus d'autorité qu'il ne leur en faudrait pour suppléer à l'insuffisance de l'individu abandonné à son initiative, ou dès que l'un d'eux en garde une portion qui pourrait être plus utile- 
ment exercée par celui qui est immédiatement au-dessous.

Cette usurpation existe-t-elle? La somme d'autorité dont jouissent ensemble la commune, le département et l'État, est-elle excessive, ou bien un de ces éléments est-il trop puissant par rapport á l'autre? Voilà les questions que soulèvent ces réclamations qui se produisent de tous les côtés, au nom de l'autonomie et de la décentralisation. Il ne s'agit pas d'organiser les choses de sorte que chaque commune, chaque département soit, dans tóute l'acception du mot, autonome, caí cela serait impossible; il s'agit de régler leurs attributions respectives. d'une manière conforme à leur intelligence et aux moyens d'action que comporterait leur initiative.

Ainsi posé, le problème est facile à résoudre en ce qui concerne le pouvoir qu'on peut laisser aux communes, relativement à la gestion des forêts qu'elles possèdent. Ėn effet, sous ce rapport, l'intelligence et les moyens d'action des particuliers et des communes sont connus. On n'a qu'à voir ce que les particuliers ont fait de leurs bois et la rapidité avec laquelle ils en opèrent le défrichement. - Dans les 50 dernières 
années, ils en ont détruit plus de 400,000 hectares. - On n'a qu'à considérer les oppositions passionnées dont l'administration publique doit triompher, afin de sauver de la ruine les forêts des communes, pour reconnaitre combien ces deux catégories de propriétaires sont inhảbiles à gérer cỏnvenablement,leurs terrains boisés; et puisqu'on ne peut plus songer à imposer une tutelle aux particuliers a u sujet de ces terrains, qu'on évite au moins de porter atteinte à celle que l'État exerce, et que seul il peut exercer utilement, sur les terrains similaires des communes.

Au surplus, ce n'est point, quoi qu'on prétende, la centralisation, c'est-à-dire la concentration entre les mains de l'administration générale du pays, d'un nòmbre exagéré d'attributions, qui par elle-même a été un embarras pour nos affaires; c'est la délégation d'une trop grande partie de ces attributions aux préfets, et c'est surtout l'usage que le pouroir exécutif a fait de son autorité dans un but politique.

Les préfets étaient devenus des espẹces de proconsuls : la tutelle des communes est encore presque entięrement entre leurs mains; leur action s'étend sur tous les services publics sans 
exception; elle embrasse, on peut le dire, tout le domaine de l'administration, en sorte que les garanties qu'on avait cherché à se procurer contre les abus de l'autorité supérieure par la division de cette autorité entre plusieurs ministres indépendants et responsables, on les a compromises en reconstituant dans la personne du préfet l'unité de pouvoir qu'avait briséé l'organisation des ministères. Il y a là une anomalie d'autant plus choquante que l'allégement des attributions ministérielles, dans l'intérêt d'une plus prompte expédition des affaires, pouvait etre facilement obtenu sans que l'on réunît sur la tête du préfet celles de ces attributions dont les ministres demandaient à être déchargés. Chaque ministre n'a-t-il pas en effet pour le représenter dans les départements, les chefs de service des administrations qui dépendent de lui ${ }^{1}$ ?

Quant à l'usage que le pouvoir exécutif a fait de son autorité dans un but politique, je

1. Au lieu d'obvier à l'omnipotence des préfets, en restituant à chacune des branches du pouvoir exécutif les attributions qui devraient lui appartenir, on a cru devoir transporter une partie de ces attributions aux conseils géıéraux, dont on a fait ainsi des corps administratifs. Je crains bien que le remède ne soit pire que le mal et qu'il n'ait affaibli l'État, sans profit pour la liberté individuelle et au grand détriment de l'intérêt public. 
me borne à rappeler que le gouvernement impérial avait conșidéré cet usage comme légitlme, et que ce fait seul était de nature à soulever les plus vives protestations. La politique, la politique intérieure, telle a été la cause principale des plaintes dont le pouvoir central a été l'objét. Elle a fait autant de mal à nos forêts que la fiscalité, et il me semble que c'est à elle que nous derons ces ardents désir's de décentralisation qui se manifestent de tous les côtés; mais la politique, si l'administration devait en avoir une à elle, ne se prêterait pas à la décentralisation; ce serait méme, de toutes les branches de l'action gouvernementale, la moins susceptible d'être fractionnée. Qu'on ne cherche pas, en coniséquence, dans la décentralisation un moyen de s'y soustraire; il y a mieux à faire que cela : il y a à l'exclure complétement du donaine de l'administration. Aussi bien, dans un pays libre, le gouvernement ne saurait suivre une autre politique que celle qui lui est indiquée par l'opinion générale sincèrement consultée.

Oui, il faut soustraire complétement à l'influence de la politique les services administratifs. Peut-être leur rendra-t-on de cette manière la considération qu'ils méritent, ce qui serait 
un inestimable bienfait et pour eux et pour le pays tout entier. En effet, le respect du fonctionnaire est une des conditions les plus essentielles de l'ordre social, et ce n'est pas la moindre de nos plaies, que ce respect n'existe plus. Les agents forestiers notamment ont à en souffrir; car c'est principalement par la persuasion, par la force morale, qu'ils peuvent accomplir leur mission. Je demanderai donc la permission de présenter à ce sujet encore, quelques observations. Elles ne seront pas hors de propos dans la préface d'un livre qui est destiné surtout à des fonctionnaires.

Il est de mode aujourd'hui, il est de bon goût de chercher à discréditer les fonctionnaires publics, et de présenter leur carrière comme une des moins honorables que puisse embrasser un homme de quelque valeur. On les met au dernier degré de l'échelle sociale. On les accable de dédains. On les regarde comme un vil troupeau qui a fait litière de toute indépendance, de toute personnalité, de toute initiative. On tient pour beaucoup plus dignes d'estime qu'eux, les individus qui conservent leur liberté d'action et ne comptent que sur eux-mémes pour se procurer les 
moyens d'existence, la fortune et la célébrité.

Ces préjugés, qui tendent à se répandre de plus en plus, ne sont pas les moins remarquables parmi ceux qu'ont engendrés la décadence des mœurs et la perversion des idées. Ils s'attaquent à la principale base de toute société, et s'ils pouvaient jamais prévaloir et éloigner des fonctions publiques les hommes intelligents, instruits, honnêtes et soucieux de leur dignité personuelle, l'état 'social serait atteint dans sa vitalité.

Qu'il y ait en France trop de fonctionnaires; qu'il y en ait beaucoup qui ne remplissent pas leur tâche d'une manière convenable; qu'on ait fait trop souvent de l'administration le refuge des gens incapables de subvenir par leurs propres forces à leurs besoins; qu'on ait usé du budget de l'État, pour avoir des créatures, tout cela n'est que trop vrai; mais on ne doit pas condamner une institution par les abus auxquels elle fournit des prétextes, et la fonction publique quand elle est bien remplie, n'en est pas moins la première de toutes les fonctions sociales, la plus enviable pour tous ceux qui tiennent à faire ici-bas un bon emploi de leurs facultés. La raison én est claire : c'est la première et la plus 
enviable de toutes les fonctions sociales, parce qu'il n'y en a aucune autre, je ne dis pas qui soit, je dis qui puisse être, aussi dégagée que celle-là de tout égoïsme. Il n'est pas indispensable pour devenir un grand industriel, un grand commerçant, un célèbre avocat, un fameux médecin, un financier cent fois millionaire, d'aimer ses semblables et son pays et de songer à leur être utile, tandis que, si petit, si humble que soit un fontionnaire public, c'est dans les services qu'il rend à ses concitoyens qu'il pouise sa raison d'être, et il ne fait rien qui ne se rapporte aux autres. Tous les sophismes du monde n'empêcheront pas cette préoccupation nécessaire de l'intérêt collectif, d'être Je caractère évident des attributions du fonctionnaire, et cela suffit pour imprimer à ces attributions un cachet particulier de distinction. Sans doute, il n'est pas défendu aux fonctionnaires publics d'avoir de l'ambition personnelle, de désirer un poste plus élevé, mieux rétribué; mais cette ambition ne peut être satisfaite que si le bien public l'est en même temps ou plutôt au préalable, tandis qu'en dehor's de l'administration, les services que l'on rend à la société ne sont que la conséquence le plus souvent 
inconsciente et involontaire de ceux que l'on se rend à soi-même.

Mais - le fonctionnaire aliène dit-on son libre arbitre. Ah! il y aurait beaucoup a dire là-dessus, et il serait facile de prouver qu'il n'y a pas de carrière qui ne comporte pour celui qui $y$, est entré, un assujetissement plus ou moins grand. Je me borne à faire observer que dans celle de l'administration publique on est subordonné à la loi, au règlement écrit, tandis que dans presque toutes les autres, on est soumis à quelqu'un, ce qui rend l'obéissance bien plus pénible pour les àmes fières. Quand on n'est l'esclare que de la loi, on a le droit de porter haut la tête; or, le fonctionnaire public peut se donner d'autres maitres, mais sa mission ne lui impose que celui-là, et c'est le seul même qu'il doire reconnaître, s'il reut la remplir dignement. Le fonctionnaire il est vrai n'est pas riche en général, et n'a aucun moyen de le devenir; raison de plus pour l'honorer. Je n'ai certainement aucun mépris pour l'homre qui dépense brillamment une inmense fortune acquise par d'heureuses spéculations; pourtant, je le mets bien au-dessous d'un simple garde forestier qui, après. s'être occupé toute la 
journée de la parcelle du domaine public dont on Iui a confié la gestion, n'a pour réparer ses forces, qu'une soupe aux pommes de terre. Jusqu'au dernier jour de sa carrière, il fera le même métier et suivra le même régime; il le sait et son zèle cependant ne se démentira pas un instant. Est-il rien de plus touchant et de plus estimable? Haussez les épaules, heureux de la terre; toujours est-il qu'auprès de ce pauvre fontionnaire, vous ne tirez pas à conséquence. Quand vous partirez de ce monde, vous n'y laisserez que des traces éphémères, rien de vous ne vous survivra. Le garde forestier dont je parlais tout à l'heure, en prenant soin de la chose publique, attache au contraire sa personnalité à dẹs travaux qui perpétueront sa mémoire. Ainsi, l'homme qui est chargé d'une fonction publique n'est pas seulement plus recommandable que les autres, parce qu'il représente de son vivant l'intérêt général, parce que la satisfaction de cet intérêt est le mobile de toutes ses actions; mais aussi, parce que sa prévoyance va au delà de sa vie temporaire, et prend autant de soins des générations futures que des générations présentes. Il est en un mot l'indispensable et le principal soutien de 
l'état social. Au surplus, je le demande à tous les esprits sincéres et impartiaux : Que serait devenue la société française, au milieu des révolutions politiques qui l'ont agitée, si elle n'avait pas eu cette administration intelligente, honnête et dérouée que l'on attaque avec tant de légèreté?

Si la France a supporté les plus terribles épreures sans y sombrer, il n'est que juste de reconnaître qu'elle le doit en grande partie à ses fonctionnaires publics. Au lendemain de la dernière révolution, lorsque le pays envahi était en quelque sorte décapité, lorsque le commerce et l'industrie étaient arrêtés, le crédit ébranlé, tous les intérêts privés épouvantés, quelle a été la conduite des fonctionnaires? Les jeunes ont pris les armes pour concourir à une défense désespérée, et les forestiers, sous ce rapport, se sont signalés entre tous ${ }^{2}$. Les autres ont continué leurs fonctions avec un redoublement de zéle; privés du jour au·lendemain, pour ainsi dire, de toute direction, ils y ont suppléé par les inspirations qu'ils ont

1. Sept jeunes agents ont trouvé une mort glorieuse dans cette cruelle guerre. Ce sont M.I. Robert, Bramand-Boucheron, Josserand,.Pison, Guérin, Moisant et Marier de Bois d'hyver. 
puisées dans leur patriotisme et dans le sentiment de leurs devoirs professionnels. Aussi, tous les grands besoins de la vie sociale n'ontils pas cessé de jouir de la protection accoutumée. Il est vrai que de leur côté les populations ont montré une résignation et une sagesse qu'on ne saurait trop admirer: mais l'anarchie n'en eût pas moins été inévitable, si cette multitude d'intérêts qui lient les citoyens à l'administration publique n'avaient pas reçu satisfaction.

Qu'on ne jette donc plus la pierre aux fonctionnaires publics. Essayer de les déconsidérer, c'est une injustice, c'est l'acte d'un mauváis citoyen. Au lieu de les amoindrir, qu'on cherche à les élever dans l'opinion des masses ignorantes et qui, parce qu'elles sont ignorantes, sont méfiantes. Qu'on cherche à les utiliser mieux qu'on ne l'a fait jusqu'à ce jour. Qu'on les épure, qu'on leur donne surtout, en améliorant leur position, la force de résister aux défaillances. Il y a dans cette voie beaucoup à faire pour tous les services, mais surtout pour celui des forêts; car il laisse grandement à désirer sous le rapport des traitements, des attributions, de la hiérarchie et du contrôle. 
Quoique ces longues réflexions ne soient point indifférentes pour la conservation des forêts, elles ne sont pas ici tout à fait à leur place. Je le sais; mais quand un homme rencontre l'occasion de plaider pro domo sud, quoi de plus naturel qu'il en profite.

Un mot maintenant sur la différence qu'il y a entre cette édition et la précédente : J'ai rêvu d'abord mon travail avec soin et je l'ai ensuite beaucoup augmenté, puisque j'y ai ajouté une étude sur les conversions dès taillis en futaie. Il n'en'est pas moins encore très-imparfait, et si je me suis décidé à le livrer tel quel à mes camarades, c'est parce que j'ai pensé qu'ils en verraient aussi bien, mieux que moi, les lacunes et les erreurs, et qu'ils en retireraient peut-être autant d'utilité par là, que par les bonnes choses qui peuvent s'y trouver. Certainement, j'espère avoir le sentiment de ce que devrait être un livre sur l'aménagement des forêts pour satisfaire à tous les desiderata que comporte la matière; mais je suis trop vieux et je n'ai pas assez de loisirs pour essayer de donner un corps à ce sentiment-là. A mon àge on ne fait guère que se paraphraser; et, d'un autre côté, mes occupations ordinaires 
ne me permeturaient pas, en supposant que mon esprit s'y prêtât, de m'absorber dans mon cuvre comme il le faudrait pour la mener.à bonne fin. On ne fait rien de sérieux, de durable dans ce monde, sans contention d'esprit; or, cette contention d'esprit à laquelle ne résistent pás les problèmes à priori les plus difficiles, n'ést guère possible quand on a des affaires courantes obligatoires et qui demandent à être traitées rapidement. Je laisse donc à des camarades plus jeunes et moins préoccupés le soin de reprendre mes idées et de les développer. Il n'en manque pas qui ont pour cela tous les moyens intellectuels et toute l'instruction nécessaires. Ce qui pourrait leur faire défaut comme à moi, ce serait le temps; mais pourquoi l'administration forestière ne détacherait-elle pas de leur service ordinaire, pour leur permettre dese consacrer à l'étude d'une question intéressant le progrès du service, les agents qui auraient montré pour cette étude une aptitude particulière? Pourquoi ne suivrait-elle pas en cela l'exemple que lui donnent les autres administrations savantes? Voilà encore une mesure que je prends la liberté de recommander à son attention et je le fais sans crainte, car je sais, par expérience, 
que l'homme éminent qui la dirige, accueille avec bienveillance toutes les propositions, pourvu qu'elles lui paraissent sincères et dictées par l'amour du bien public.

L. TASSY.

Paris, arril 1872. 


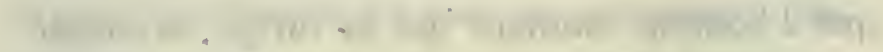

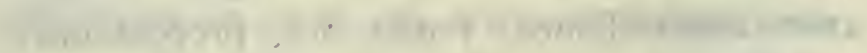

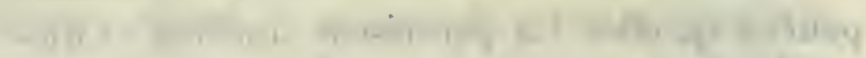

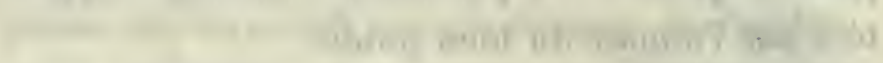
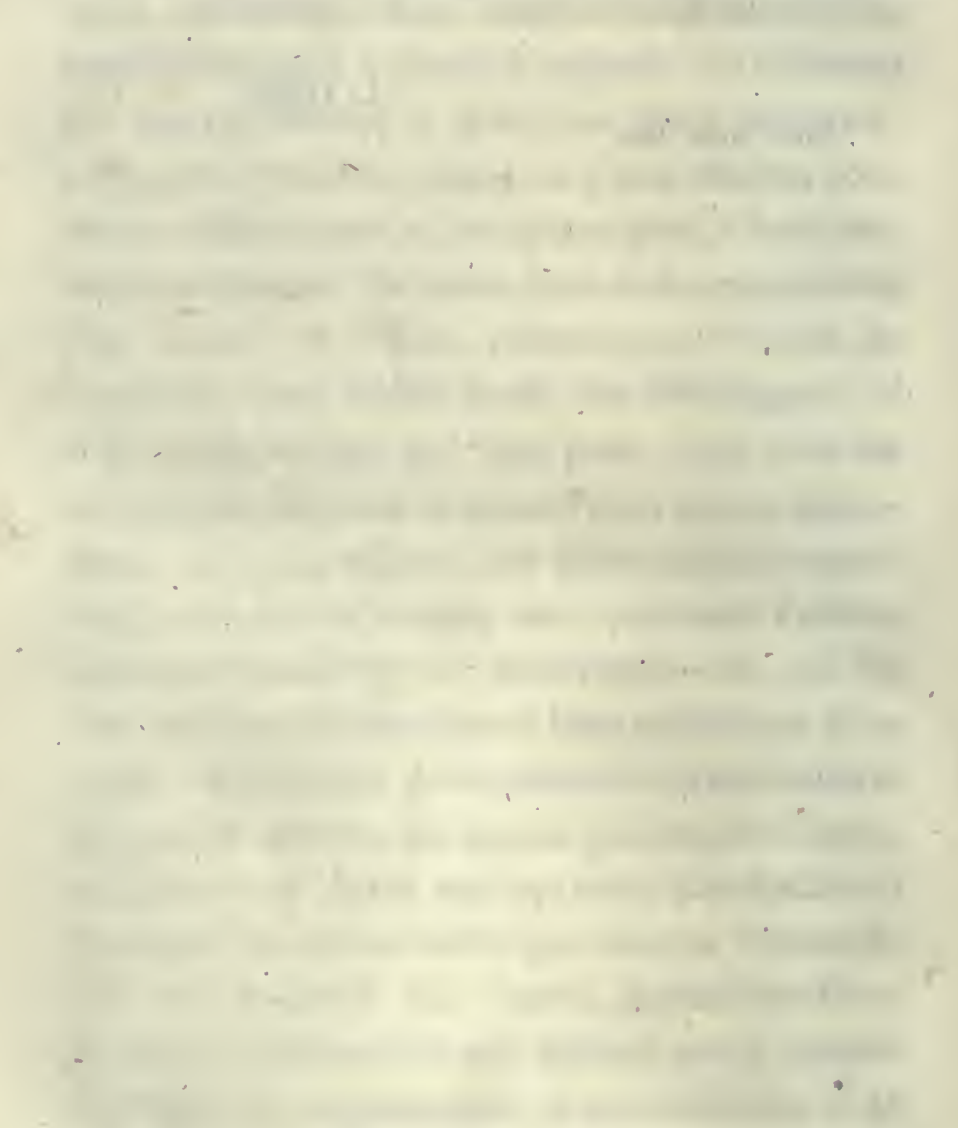


\section{PRÉFACE}

DE LA PREMIÉRE ÉDITION.

A l'époque où la civilisation romaine fut apportée dans les Gaules par Jules César, ce pays était presque entièrement couvert de bois ${ }^{1}$.

Parmi les forêts qui couvraient alors notre territoire, la plus remarquable par son étendue était celle des Ardennes (la forêt profonde); elle arait plus de cent lieues de long. Ces rastes déserts qu'on appelle la Sologne, la Dombes, la Brenne, les Landes, les Dunes, éfaient boisés. La ville de Paris, l'ancienne Lutèce, était entourée de forêts, dont celles de Boulogne, de Montmorency, de Vincennes, de Fontainebleau, ne sont que les restiges.

De ces richesses, il nous reste à peine huit millions d'hectares, dont la plus grande partie en taillis simples, clairiérés, n'offrant quelque intérêt que pour le chauffage.

1. Le mot Gaule paralt etre d'invention romaine; les gens du pays l'appelaient Celtique. Toutefois, quoique le mot Gallia soit latin, il vient éridemment du mot celtique gaél, forêt (Ozaveaux). 
Toute la région comprise entre le Rhône, la Méditerranée et les Cévennes, est presque entièrement dénudée. Il en est de même des côtes de l'Océan et de tout le plateau central.

Quelles sont les causes de cet appaurrissement?

Quand on songe à la violence et à la durée des guerres dont notre pays a été le théâtre, et qu'on se reporte à cette lutte effroyable de dix années qu'eut à soutenir Jules César pour en achever la conquête; quand on se rappelle que si, depuis cette conquête (60 ans avant J. C.) jusqu'à l'invasion franque (406), la Gaule jouit d'une tranquillité relative, vingt peuples divers, sortis des profondeurs de la Germanie, se précipitèrent alors sur elle, et qu'il y eut un désastre immense, universel; que quarante ans plus tard (451), de nouvelles hordes vinrent fondre encore sur cette malheureuse contrée à la suite d'Attila ; qu'en 711, les Arabes, à leur tour, franchissant le détroịt, y portèrent le fer et la flamme; quand on réfléchit à la puissance dévastatrice de ces multitudes en mouvement, on se dit que c'est là qu'il faut chercher la cause principale du déboisement actuel de notre sol. On ne l'y trouverait cependant pas.

Il paraît en effet certain que peu de temps après ces affreuses guerres qui ont rempli les premiers siècles de notre histoire, la végétation forestière avait repris une grande partie du terrain qui lui avait été enlevé. Et cela s'explique : la guerre, avec. les fléaux qu'elle traînait à sa suite, la peste et la famine, occasionnait d'épouvantables mortalités parmi les hommes et dépeuplait les campagnes. Les terres, abandonnées par la culture, étaient de nouveau envahies par les forêts.

Des ruines qui ont été découvertes dans les forêts du 
Haut-Rhin, dans celles de Grand (Vosges), de Damville (Meurthè), et qui remontent à l'époque romaine, indiquent que le sol de ces forêts était autrefois cultiré. Des ruines également romaines existent à la PetiteHoussaye dans la forêt de Brotonne, en Normandie, dans la forêt de Beaumont-le-Roger (Eure). Le plateau de Linenberg, près Abrescheviller, en Lorraine, jadis en culture, est aujourd'hui entièrement boisé ${ }^{1}$. Au quinzième siècle, c'était un 'dicton populaire en France que les guerres des Anglais ! avaient fait pousser le bois?

Ce n'est donc pas la guerre qui a été la cause principale de l'appaurrissement dont le sol forestier souffre aujourd'hui; c'est la civilisation arec les besoins qu'elle a créés et les appétits qu'elle a développés.

Je n'apprendrai rien assurément à mes lecteurs en leur disant que les hommes ont puisé dans les forêts les premiers éléments de leur bien-être et de leur émancipation : a l'origine, ils vivaient surtout des fruits saurages et du produit de la chasse. Les bois leur fournissaient aussi et les abris grossiers dont ils se.contentaient et les armes dont ils se servaient. Plus tard, quand ils connurent l'art de domestiquer les animaux, ils devinrent pasteurs ; ils franchirent le premier degré de l'échelle de la civilisation et ce progrès eut pour conséquence forcée et immédiate, le défrichement d'une certaine étendue de forêts : on dut créer des pâturages. Enfin, l'agriculture fut inventée, et à partir de ce moment, la variété et la quantité toujours croissantes des matières alimentaires farorisèrent l'aug̉mentation de la population, provoquèrent de noureaux besoins. Le

\section{- 1. Macky, Histoire des foréts.}

2. Histoire de France, Hexri Martix. 
sol était conquis ; il fallait conquérir les eaux; les forêts en fournirent encore les moyens. Elles suffirent à toutes les exigences; on les attaqua de tous les côtés et par tous les motifs : lantot, parce qu'elles étaient un obstacle à la culture des céréales, tantôt parce qu'elles renfermaient des instruments de travail, de défense ou de transport.

Il serait bien difficile de déterminer l'effet de chacune de ces influences, et d'ailleurs cela n'est pas nécessaire : je veux établir seulement que les besoins de la civilisation ont puissamment contribué à la destruction des forêts; je tiens à prouver ensuite que ces besoins ont rencontré malheureusement d'énergiques auxiliaires dans les vices que la civilisation engendre, quand elle se laisse emporter par l'orgueil et qu'elle tombe dans le matérialisme. Parmi ces vices, je signalerai l'oubli de la solidarité humaine et l'imprévoyance qui en est la conséquence, le goût des plaisiıs physiques, l'ardeur pour les richesses immédiatement réalisables, l'indifférence pour celles qu'il faut attendre.

L'homme de uos jours ne se soucie ni du passé ni de l'avenir ; il se soúcie du présent, et dans le présent il ne voit que lui. Il a perdu le culte des ä̈eux; le sort de ses descendants ne le touche pas et depuis longtemps il ne connaît plus les joies sublimes du sacrifice et du dévouement. Il ne vit que pour la satisfaction de ses appétits égoïstes. Toutes ses aspirations étant concentrées dans l'amour de soi-même, on comprend son dédain pour les biens dont il he saurait jouir incontinent et qui pourraient dès lors lui échapper.

La conservation des forêts est inconciliable avec de telles dispositions morales : sans le respect des traditions, sans l'esprit de prévoyance, sans l'intelligence des 
deroirs réciproques que les générations ont à accomplir, sans l'amour de ses semblables, il n'y a pas de garantie sérieuse pour cette conserration. Le profit immédiat que le défrichement d'une forêt procurerait, prévaudra un jour ou l'autre sur les avantages qu'on assurerait à l'avenir, en la laissant sur pied.

Cependant, l'ignorance trop généralement répandue dans laquelle on est, en France surtout, relativement à ces arantages, donne, - jaime à lè supposer, - aux causes de destruction que je viens d'énumérer, une force particulière, et je ne désespère pas assez de mon payss, pour croire que s'il était bien pénétré des dangers auxquels il s'expose par l'anéantissement de ses forêts, il continuerait à les braver avec tant d'insoucianco.

La vịe des hommes est attachée à celle des arbres, on l'oublie trop. Parmi lés nations civilisées et rivales, la France est déjà une de celles qui possèdent le moins de bois, motif d'infériorité plus grave qu'on ne le pense. La Provence se dépeuple, parce que les forêts disparaissent ${ }^{1}$. Nous payons un tribut annuel de plus de soixante-dix millions de francs à l'étranger pour combler le déficit de notre production². Nous possédons une magnifique flotte, mais une bataille navale malheureuse peut nous l'enlever, et nous la remplacerions difficilement. La situation mérite qu'on y prenne garde et qu'on s'occupe d'y remédier ${ }^{3}$.

Quand on lit l'histoire des anciens; quand on étudie

1. La Provence au point de vue des bois, des torrents, CaAnles DE RibBe.

2. Ce tribut est aujourd'hui de 150 millions. (Note de la $2^{e}$ édition.

3. Je sais bien que l'administration forestière fait dans ce but les plus louables efforts; malheureusement, son action est très-limitée. 
leur caractère, on les voit tout pleins d'un respect religieux pour les bois. Cette histoire est remplie de traits qui montrent qu'une superstition tutélaire s'attachait autrefois à l'existence des arbres; ils étaient placés sous la protection spéciale de la Divinité. Nos mœurs sont bien éloignées de celles de nos pères; notre raison impitoyable a voulu sonder toưs les myștères; elle a fait tomber tous les prestiges, et pour ce qui concerne les bois, leur conservation serait tout à fait compromise si elle ne reposait que sur la vénération dont ils sont l'objet. On peut dire même que, de toutes les propriétés, c'est la moins respectée. Puisqu'il en est ainsi; puisque le positivisme de notre époque a renversé les barrières que le sentiment religicux opposait jadis à l'esprit de destruction, on doit chercher dans l'intérêt des populations une sauvegarde qui remplace celle qui n'existe plus. Il faut faire comprendre les avantages économiques inhérents à la propriété boisée; il faut surtout enseigner les moyens d'en tirer tous les fi'uits désirables.

Pour les personnes étrangères à la sylviculture, la forêt vierge représente le type du beau en matière de richesse forestière : elle est l'expression la plus haute des ressources que peut fournir une masse d'arbres.

Il y a dans cette opinion une crreur grossière:

Les forêts, comme les autres biens que la Providence a répandus sur notre globe, ont besoin des soins de l'homme pour développer toute leur puissance productive : là comme ailleurs, la terre n'est féconde que pour celui qui la cultive. Le tout est de la bien cultiver, et il est en conséquence désirable qu'on en vulgarise l'art autant que possible. 
C'est, pour mon compte, ce que je viens essayer de faire. J'ai eu ả remplir des attributions qui m'ont forcé d'étudier avec une attention particulière une partie de la science forestière sur laquelle il n'existe en France qu'un très-petit nombre d'écrits. Je reux parler de l'aménagement. J'ai pensé que mon devoir était de publier le résultat_de mes travaux. On n'y trourera pas beaucoup de nouveautés : la plupart des idées exposées dans ce volume sont connues, mais il en est peu qui aient été imprimées. Je les ai recueillies; je les ai groupées aussi méthodiquement que j’ai pu : voilà mon seul mérite. Parmi les agents, et ils sont nombreux, qui auraient le droit de revendiquer comme leur appartenant plusieurs dẹs principes et des considérations que je vais développer, je dois en citer poultant deux :

II. Parade, directeur de l'École impériale, forestière;

M. de Buffévent, ancien conservateur des forêts.

Ces deux hommes éminents qui roulaient bien m'honorer de leur amitié, m'ont fourni beaucoup de notions, beaucoup de matériaux, soit par leurs ouvrages, soit par leurs entretiens.

Une dernière observation : Je prie que l'on fasse attention au titre de ce volume : Éludes sur l'aménagement. Je ne prétends pas publier un traité. Il y a á l'École de Nancy un professeur fort distingué, M. Nanquette, à qui je laisse ce soin, soin dont il s'occupe, mais qui demande beaucoup de temps, et dont il s'acquittera mieux que je n'aurais pu le faire ${ }^{1}$. Je publie de simples

1. Ce traité a paru avec l'entière approbation de M. Parade. Il ne pouvait avoir une meilleure recommandation. 
XXXIV PRÉFACE DE LA PREMIËRE ÉDITION.

études, un essai, un programme, par conséquent quelque chose d'incomplet et de discutable. Qu'on veuille bien seulement me discuter, et je n'aurai pas perdu mon temps.

L. T.

Paris, 1858. 


\section{INTRODUCTION}

L'aménagement est un travail qui consiste à régler l'exploitation d'une forêt, de façon qu'elle fournisse un rapport annuel aussi soutenu et aussi avantageux que possible ${ }^{1}$.

L'homme a des besoins incessants, dont les principaux, ceux qui intéressent son existence matérielle, réclament impérieusement une satisfaction immédiate. Pour y subvenir, il lui faut des objets de consommation constamment disponibles; de là sa tendance à obtenir des agents de production qui sont à sa portée, une continuité de rendement.

Cette tendance est surtout manifeste dans les

1. J'ai cru devoir maintenir cette définition, quoiqu'elle diffêre de celle que donne le cours de culture. D'après ce cours, l'aménagement consiste à régler l'exploitation d'une forêt dans le plus grand intérêt du propriétaire et de la consommation. Or, la condition du rapport annuel n'est pas comprise dans cette définition, et elle est pourtant, selon moi, caractéristique de l'aménagement. 
sociétés primitives : on y vit, au jour le jour, du produit de la pêche, de la chasse, etc., etc. Au milieu des ressources qui l'environnent, l'homme n'y apprécie guère que celles-là, parce que ce sont presque les seules dont l'exploitation puisse se concilier avec le renouvellement journalier de ses besoins. Le sol, défriché et ensemencé, lui fournirait sans doute, au bout de l'an, dix fois plus de matière alimentaire que ne lui en procurent la chasse et la pêche. Peu lui importe; il ne saurait rester un an sans manger. Mais quand, à force de persévérance, il est parvenu à réaliser quelques économies sur les menus profits de son travail, et qu'il s'est mis de cette façon à même de soutenir son existence pendant le temps nécessaire à la culture du sol, alors il a fait un grand pas dans la voie du progrès. A partir de ce moment, il lui est permis d'utiliser d'autres ressources, et de concilier avec des besoins qui se renouvellent, pour ainsi dire, à chaque instant, des cultures dont les produits ne sont réalisables que périodiquement, à des intervalles plus ou moins longs.

L'épargne, c'est-à-dire la mise en réserve d'une certaine quantité de produits, qui permettent d'attendre, voilà donc le moyen nécessaire auquel les hommes ont dû recourir pour approprier à la satisfaction de leur's besoins, les productions intermittentes; voilà le progrès qui explique la valeur 
que l'on attribue, dans les sociétés civilisées, à des agents, à des capitaux dont les produits ne seront disponibles que dans un avenir plus ou moins éloigné. Toutefois, ce progrès n'est pas susceptible de déplacer le pivot autour duquel gravite la production, ni de modifier la condition qui sert de régulateur à cette dernière, qui la dirige après l'avoir provoquée. Les besoins de l'homme n'en restent pas moins incessants, et, par une conséquence forcée, les productions réglées de la manière la plus avantageuse à ses intérêts, sont celles qui présentent le plus de continuité.

L'épargne, lorsqu'elle est sous la forme d'objets de consommation, a, d'ailleurs, deux inconvénients graves :

D'abord, elle ne se conserve pas longtemps.

Le froment, par exemple, ne tarde pas à se détériorer dans un grenier. Le bois subit le même sort, quand il est exploité depuis un certain nombre d'années. Une récolte de blé ou de bois qui n'aurait lieu que tous les vingt ans, ne vaudrait donc pas, en supposant qu'elle fût vingt fois plus considérable, celle qu'on pourrait faire chaque année.

L'autre inconvénient des produits accumulés par l'épargne, c'est de solliciter l'imprévoyance, le gaspillage, et d'entraîner, pour le moins, une consommation excessive.

Les raisons précédentes sont suffisantes pour 
justifier la transformation des capitaux dont les. produits se succèdent à de longs intervalles, en capitaux dont les produits sont annuels, et si le consommateur est grandement intéressé à cette transformation, le propriétaire du capital n'est pas appelé à en retirer de moindres avantages.

C'est un fait bien connu que les branches d'industrie le plus favorablement placées, au point de vue des bénéfices qu"elles offrent à ceux qui les exploitent, sont celles qui peuvent le plus facilement se plier aux fluctuations de la demande; or, entre deux forêts dont l'une, aménagée, serait susceptible d'un rapport annuel et soutenu, tandis que l'autre, non amẻnagée, ne serait exploitable qu'à des intervalles relativement fort longs, il est évident que c'est la première dont la production se subordonnera le plus aisément à ces convenances dont je viens de parler, à ces mouvements de hausse ou de baisse dans la demande du consommateur. Ce qu'il faut, en effet, pour cela, c'est une disponibilité constante de produits exploitables ou sur le point de l'être. L'aménagement peut seul la fournir.

$\mathrm{Au}$ reste, ce budget annuel des dépenses et des recettes, qui est entré dans les habitudes de tous les hommes, prouve surabondamment les avantages de l'annúalité, quel que soit le revenu.que l'on considère. A l'expiration de l'année, chacun, l'individu comme la commune, comme l'État, chacun 
arrête ses dépenses, opère ses rentrées, et il en résulte que de toutes les rentrées, celles-là sont naturellement le plus désirables, qui correspondent à l'époque de ce bilan, sans lequel les règles de l'économie privée ou collective seraient fort difficiles à appliquer.

Le consommateur et le producteur sont donc tous les deux d'accord pour que les forêts soient exploitées sous la condition d'un rapport annuel et soutenu; cependant ils sont bien loin de comprendre la réalisation de ce rapport de la même manière, et la divergence de leurs sentiments, sur ce point, est importante à noter.

Le consommateur ne demande qu'une chose: c'est que l'exploitation de toutes les forêts qui fournissent à ses besoins soit combinée de telle. sorte que, chaque année; elle lui offre les mêmes ressources. Il ne se soucie pas du règlement auquel chacune de ces forèts, considérée séparément, pourrait être assujettie. Si l'on suppose qu'elles soient au nombre de 30 , qu'elles appartiennent à des propriétaires différents, et qu'elles soient exploitables à 30 ans, le consommateur admettra volontiers qu'aucune d'elles ne soit aménagée, si d'ailleurs il y a, dans leurs âges, une gradation qui empêch c que l'exploitation de l'une ne coincide avec celle de l'autre. Le rapport soutenu n'en existera pas moins, pour l'ensemble de ces forêts, et c'est là tout ce que le, consommateur réclame. 
Le producteur n'envisage pas ainsi les chọses ; ce qu'il veut, c'est que le rapport soutenu soit établi dans la forêt dont il est propriétaire; ce que font ses voisins ne le touche que très-indirectement.

Le consommateur désire des produits soutenus, non-seulement en matière, mais en marchandises de telle ou telle espèce.

Le producteur ne recherche, en général, que le rendement en argent, quand ce producteur est dans la catégorie des particuliers. S'il s'agissait d'un État, d'une communauté quelconque, il en serait autrement, parce que leurs intérêts s'identifient plus complétement que ceux des particuliers avec les intérêts du consommateur.

Ces différences sont essentielles et soulèvent des questions sur lesquelles il y aurait certainement encore beaucoup à dire; mais les développements dans les quels je pourrais entrer à ce sujet seraient sans utilité aujourd'hui. Je me bornerai à ajouter aux principes qui découlent des considérations précédentes, que si l'établissement du rapport soutenu est une condition que les propriétaires de bois doivent, ordinairement, chercher à réaliser, il y a cependant, pour les particuliers, des cas où il ne leur convient pas de le faire. La petite étendue d'un bois peut être une raison poúr qu'on ne l'exploite pas en plusieurs coupes successives, parce que ces coupes occasionneraient des frais généraux plus considérables que ceux qu'en- 
trainerait l'exploitation en une seule fois, et ne seraient pas suffisamment compensés par les avantages de l'annualité.

En outre, les propriétaires sont quelquefois conduits, par des molifs d'économie domestique, à considérer un bois comme une caisse d'épargne, dans laquelle ils désirent accumuler, pour une certaine époque, pour le mariage d'une fille, pour l'établissement d'un garçon, etc., etc., des valeurs plus ou moins importantes. C'est encore une raison pour que ce bois ne soit pas aménagé.

Un propriétaire peut enfin être sollicité par une hausse extraordinaire du prix des bois, ou par la nécessité de faire face à des engagements, à réaliser la superficie entière ou presque entière de la forèt qui lui appartient.

Ce sont là des exceptions; elles ne sont jamais, sauf la première, admissibles pour l'État, pcur une commune ou un établissement public, pour les corps impérissables enfin, dont le devoir est de distribuer, aussi également que possible, aux générations successives dans lesquelles ils sont destinés à se perpétuer, les fruits et les richesses dont ils disposent.

Mais la régularisation du rendement annuel est surtout obligatoire pour les forèts de l'État; car leurs produits forment le pain quotidien d'une multitude d'industries et ne peuvent varier, sans qu'il en 
résulte de grandes perturbations et d'incalculables souffrances.

En présence des fluctuations auxquelles ce rendement est resté soumis jusqu'à ce jour, il n'y a pas d'usine employant le bois comme principal combustible, dont il soit possible d'apprécier d'une manière exacte les conditions de roulement, et une des causes principales de la situation précaire dans laquelle se trouve la métallurgie au bois et des plaintes ardentes dont elle ne cesse d'assiéger l'administration, réside dans la variabilité des produits que celle-ci met en vente chaque année.

Les particuliers propriétaires de bois ne sont pas moins intéressés que les consommateurs à ce qu'il * soit mis un terme à cet état de choses. Aujourd'hui, l'administration peut à son gré faire la baisse ou la hausse par la quantité de bois plus ou moins considérable qu'elle jette sur le marché. Tant que cette quantité ne sera pas réglée, et elle ne le sera que par l'aménagement, les particuliers seront exposés au danger permanent d'une concurrence susceptible d'avilir, à un moment inattendu, le prix de leurs coupes. Ce danger entrave leurs opérations et déprécie leurs immeubles.

L'aménagement répond, on le voit, à l'une des exigences les plus légitimes des sociétés civilisées, quand il a pour but d'annualiser et de régulariser la production du sol forestier. 
Mais ce résultat n'est pas le seul qu'il soit propre à procurer.

Depuis l'époque où une forêt est susceptible de se régénérer naturellement, soit par les semencès, soit par les souches, elle peut donner autant de rapports soutenus différents qu'il y a d'années à courir jusqu'au moment de son dépérissement. Il suffit, en effet, pour assurer la perennité de sa production, de n'en extraire chaque année qu'une quantité de bois égale à son accroissement moyen; or, parmi ces rapports soutenus, il y en a nécessairement un qui est préférable à tous les autres : c'est celui dont l'expression, soit par contenance, soit par volume, forme ce qu'on appelle la possibilité d'une forêt. L'aménagement a pour résultat de le déterminer, et ce résultat n'est pas moins recommandable que le précédent.

Je viens de justifier le but de l'aménagement, je, vais indiquer succinctement quelles sont les opérations à faire pour l'atteindre :

Quand on veut retirer d'une masse de bois un produit annuel constant et non interrompu, il faut la partager en un certain nombre de coupes annuellement et successivement exploitables, de manière que le repeuplement de l'une quelconque de ces coupes puisse devenir exploitable à son tour, dans le temps nécessaire pour régénérer les autres.

Ce partage d'une forêt en coupes; la fixation de 
l'ordre dans lequel lesdites coupes devront être exploitées, forment ce qu'on appelle le plan d'exploitation. Le temps à courir afin qu'une coupe revienne unè deuxième fois en tour d'exploitation, est ce qu'on nomme la révolution.

L'établissement du plan d'exploitation est l'opération essentielle de l'aménagement.

Cet établissement est subordonné à la nature des produits que l'on recherche, et à l'âge auquel il convient d'abattre les arbres pour' qu'ils soient réalisés. Si l'on destine une forêt à la production des cercles, on devra certainement l'exploiter à un âge beaucoup moins avancé, dans une révolution beaucoup plus courte, que si on la destine à la production de bois propre aux constructions.

L'âge qui correspond au rapport le plus avantageux, et le plus avantageux est celui qui convient le mieux au propriétaire, est ce qu'on appelle l'age d'exploitabilité.

La détermination de cet age d'exploitabilité précède donc nécessairement la formation du plan d'exploitation.

Mais l'âge d'exploitabilité d'une forêt est trèsvariable, suivant la position du propriétaire, ses besoins, les conditions tant naturelles qu'économiques dans lesquelles est placée la forêt, et ces conditions, il est indispensable de les connaitre.

La reconnaissance de la forêt, sa description, 
l'inventaire de toutes les circonstances qui pourraient exercer une influence sur sa conservation et son rendement, sont donc les objets dont on doit s'occuper avant tout autre, lorsqu'on entreprend un aménagement.

La réunion des divers renseignements que ces objets comportent, constitue l'opération connue sous le nom de statistique.

La statistique,

La recherche de l'age d'exploilabilité,

La formation du plan d'exploitation,

Telles sont, en définitive, les opérations principales qu'exige un travail d'aménagement.

Il convient en outre d'y ajouter l'étude et la proposition de tous les travaux d'ámélioration qui seraient propres à augmenter le rendement.

L'aménagement est un travail très-simple, en général, pour les bois de particuliers, difficile, quelquefois, pour ceux des communes, toujours trèscompliqué pour les forêts de l'État.

Dans toute société arrivée à un certain degré de civilisation, il s'est établi un mouvement commercial et industriel contre lequel un particulier ne saurait réagir; il est forcé de s'y soumettre quand il aménage ses bois. Qu'il s'agisse, par exemple, de l'écoulement à donner à leurs produits, cet écoulement est déterminé par des débouchés, par des voies qui existent déjà en vertu d'une foule de causes plus ou 
moins fondées, plus ou moins légitimes, mais dont il faut bien qu'il accepte les conséquences. Il y a là une première raison, raison capitale, pour que l'aménagement des bois de particuliers soit en général un travail facile à faire; il l'est en outre, parce que ces bois sont tous assujettis à de courtes révolutions et à une méthode de culture d'une application commode.

L'aménagement des bois des communes est plus embarrassant : une commune n'est pas dépourvue de toute influence sur les conditions 'générales dans lesquelles se meut la société dont elle fait partie; elle peut, dans une certaine mesure, réagir sur elles. D'ailleurs, ce n'est pas seulement par le produit net qu'elle se laisse guider, comme le font en général les particuliers, lorsqu'elle règle l'exploitation de ses bois; elle consulte aussi l'avantage d'un produit brut plus élevé; elle embrasse enfin plusieurs générations et doit songer à l'intérêt de l'avenir comme à celui du présent.

Ce sont tout autant de considérations qui augmentent l'importance et les difficultés de l'aménagement des bois communaux. Ajoutons que le domaine forestier d'une commune occupant ordinairement plus d'étendue que celui d'un particulier, est par cela même moins aisé à mettre en ordre dans un but déterminé; que les révolutions auxquelles le premier eșt soumis, sont plus longues; que le mode d'exploitation auquel il est assujetti, est plus exigeant. 
Quant aux forêts domaniales, ce qui en complique l'aménagement, ce n'est pas seulement le nombre et la variété des intérêts qu'il doit concilier; c'est encore et principalement la possibilité de modifier les conditions économiques générales dans lesquelles il est appelé à fonctionner.

Les routes par lesquelles se sont exportés jusqu'à ce jour, les produits de la forêt $\mathrm{F}$, ne conduisent qu'à des centres de populations agricoles. S'il n'y avait pas moyen de créer d'autres débouchés à cette forêt, il serait sans objet d'y favoriser la production de bois de fortes dimensions; mais on pourrait facilement la rattacher par un canal à un chantier de constructions navales; cette possibilité méritera d'entrer dans les considérations qui serviront à régler son aménagement.

Je m'en tiens à cet exemple; il n'est pas nécessaire d'en donner d'autres pour prouver que lorsque l'État aménage ses forêts, il est tenu de se préoccuper des modifications qu'il conviendrait d'apporter, dans l'intérêt de leur exploitation, au milieu qui les entoure, et que ce devoir -comporte des appréciations d'un ordre élevé, très-complexes , très-délicates.

D'après les définitions que j'ai données de l'aménagement, du but qu'il poursuit et des opérations qu'il nécessite, il est facile de voir qu'il y a entre cette science et la culture proprement dite, des dif- 
férences bien tranchées; que la culture puise tous les principes qui la constituent dans les faits naturels qu'elle se borne à classer d'une manière rationnelle; tandis que l'aménagement traite des moyens d'approprier ces faits aux usages de la société; que la première est une science d'observations, tandis que la seconde est une science de combinaisons.

La théorie occupe donc une place plus grande dans l'aménagement que dans la culture, et je crains que ce ne soit pour beaucoup de gens un titre de défaveur. Qu'on me permette à ce sujet quelques courtes réflexions.

On affecte aujourd'hui de poursuivre comme inutiles, stériles sinon dangereuses, les théories scientifiques. C'est encore un effet de la tendance matérialiste de notre époque. A en croire certaines personnes, il n'y aurait de sérieux, de réel, d'utile, que la pratique. A les entendre, le dernier des gâcheurs de plâtre, des tailleurs de pierre, des cantonniers ou des bûcherons, en saurait plus pour la construction des maisons, l'entretien des routes, l'amélioration et l'exploitation des bois, que les hommes qui, avant de se livrer à la pratique des arts, en ont étudié la théorie.

Ce sont là des énormités qui ne supportent pas l'examen.

S'il est vrai que la théorie soit la raison des choses, l'explication des phénomènes de la nature et 
l'énoncé des, règles, des méthodes à suivre paur faire servir ces phénomènes à la satisfaction des besoins de l'humanité; s'il est vrai que la pratique ne soit au contraire que l'application de ces règles, de ces méthodes, n'y a-t-il pas, je le demande, entre la théorie et la pratique une union nécessaire, qu'il serait tout aussi difficile de rompre que de séparer la main qui agit de l'esprit qui la dirige?

Maintenant, je le reconnais, il ne suffit pas de savoir comment une opération doit se faire, pour être capable de l'exécuter. Les travaux matériels demandent une aptitude, un tact, une adresse, un tour de main dont on ne saurait contester l'utilité, et qui ne s'acquièrent que par l'expérience; mais toujours est-il que cette expérience se formerait plus difficilement, plus lentement, si elle n'était guidée que par l'instinct au lieu de l'être par le raisonnement.

Il est vrai que la théorie ne pose guère que des principes généraux; qu'elle n'a pas de règles de conduite pour toute les exceptions, toutes les anomalies; qu'elle ne dispense personne de faire usage de son esprit d'initiative, de sa raison, de ses observations personnelles; mais toujours èst-il qu'en enseignant les lois générales qui régissent les divers ordres de faits, elle rend plus sûre la résolution des cas particuliers qu'elle ne saurait prévoir; car il n'y a pas de cas, quelque particulier qu'on le suppose, 
qui soit sans aucun rapport, sans aucun lien, sans aucune analogie avec une circonstance typique.

Abandonnez la sylviculture aux simples ressources de la pratique, et vous la réduisez, comme l'ont dit MM. Lorentz et Parade dans l'introduction de leur Cours de culture, à une routine incertaine et obscure. Il ne saurait en être autrement dans un ordre de choses, où les faits mettent plus de temps à se produire que l'homme n'en met à accomplir sa destinée.

Concluons donc que si la pratique doit être considérée, en économie forestière surtout, comme un élément indispensable de succès, elle ne-saurait cependant aboutir qu'à de médiocres résultats si elle n'était éclairée par la théorie.

Concluons qu'en sylviculture comme en toute autre matière, sans le secours de la théorie, le niveau des connaissances humaines ne pourrait jamais s'élever; puisque c'est à elle qu'il appartient d'étudier les phénomènes, de les grouper, d'en constater les lois, et de faire profiter ainsi une génération de l'expérience de celles qui l'ont précédée. 


\title{
ÉTUDES
}

SUR

\section{L'A MÉN A GEMENT}

\author{
DES FORETS
}

\section{PREḾI ÈRE ÉTUDF.}

DE LA STATISTIQUE.

\section{CHA PITRE PREMIER.}

DE LA STATISTIQUE EX GEYÉRAL, ET DES ËTUDES

$$
\text { QU.'ELLE COMPORTE }
$$

AU POIST DE VUE DE L'AMÉNAGEMENT.

La statistique est, à un point de vue général, l'inventaire, dans un ordre méthodique, de tous les faits qui concernent les besoins de la société et le développement de ses richesses.

Elle n'est pas seulement utile ; elle est indispensable au progrès de la civilisation.

Vous êtes à la tête du gouvernement; vouś vou- 
lez doter votre pays d'un réseau de voies de communication; pour tracer ce réseau de la manière la plus convenable, il faut connaître les exigences de la consommation. Ces exigences, la statistique vous permettra d'en apprécier l'étendue.

Tous les travaux de l'homme, petits ou grands, toutes ses spéculations, quelle qu'en soit l'importance, s'appuient nécessairement sur la statistique.

L'industrie forestière n'est point affranchie de cette obligation. Elle y est même astreinte plus étroitement que toute autre, sous peine de manquer le but qu'elle doit se proposer d'atteindre. Je vais en citer quelques preuves :

L'administration dispose d'une certaine somme pour des améliorations, pour des travaux de route, par exemple. En réunissant les demandes de crédit, faites à ce sujet par les chefs de service, on arrive à un chiffre de dépense qui dépasse considérablement les ressources disponibles. Il est danc nécessaire de rejeter quelques-unes de ces demandes, de n'en accueillir d'autres qu'en partie, d'aller enfin au plus pressé. L'administration serait dans l'impossibilité de procéder avec discernement à cette délicate besogne, si elle n'était éclairée sur les localités où la construction des voies de vidange est la plus urgente; mais en jetant les yeux sur les données de la statistique, elle voit que dans telle localité, le coût du transport s'élève à la moitié du prix vénal des bois; que dans telle autre, au contraire, il ne s'élève qu'au tiers. C'est par conséquent dans la 
première qu'il est pressant d'améliorer ou de créer des chemins, et c'est là qu'elle porte ses moyens d'action. Le manque de renseignements statistiques l'exposerait évidemment à dépenser ses ressources pécuniaires dans les régions qui en auraient le moins grand besoin.

Depuis longtemps déjà, on donne chaque année à l'administration 7 à $\$ 00,000$ francs pour être affectés à des repeuplements artificiels. C'est une forte somme qui, bien employée, doit amener de beaux résultats; mais il ne faut pas croire que pour l'utiliser il suffise d'acheter des graines quelcońques, et de les jeter au hasard dans les premiers vides qui se présenteront. Suivant la position qu'elles occupent, les forêts réclament des essences particulières. Si elles sont situées, par exemple, non loin des fleuves qui aboutissent dans le voisinage de nos arsenaux maritimes, il est désirable qu'on y propage les espèces propres aux constructions navales; si elles sont situées près des routes qui conduisent à de grands centres de population, il convient d'y élever des arbres propres à la menuiserie, à l'ébénisterie, aux construclions civiles, etc., etc.; enfin, il est nécessaire, avant tout, que les essences soient appropriées au climat et au sol. Dans ces cas-ci comme dans le précédent, la statistique est un guide indispensable pour ne pas se fourvoyer.

Il résulte deces considérations préliminaires qu'une statistique générale, embrassant tous les éléments qui se rattachent au développement et à l'emploi des 
richesses forestières de la société, est un répertoire essentiel à défaut duquel la gestion de ces richesses ne saurait être réglće d'une manière rationnelle.

Il est à remarquer d'ailleurs qu'une forêt ne forme pas un ubjet qu'il soit permis de considérer isolément; qu'elle est solidaire de toutes celles qui se trouvent dans le même pays, ou qui sont destinées à alimenter les mêmes centres de consommation; que lorsqu'on aménage une forêt des Vosges, il y a lieu de se préoccuper des conséquences de cet aménagement pour les forêts de la Lorraine, et vice versâ.

En résumé, l'exploitatión des forêts est subordonnée à des conditions générales d'économie agricole, industrielle et commerciale, que les aménagistes doivent connaitre, et ces conditions, il appartient à la statistique de les définir.

Mais quand on a réuni, et je suppose qu'on l'ait fait en France, les notions générales et fondamentales dont je viens de parler, il reste à mettre l'exploitation de chaque forêt, prise à part, envisagée séparé-• ment, en harmonie avec les circonstances spéciales, naturelles ou économiques, au milieu desquelles elle se trouve placée, qui la touchent directement, et c'est par rapport à ces circonstances spéciales que j'entends le mot placé en tête de ce chapitre et que je vais essayer d'indiquer toutes les études qu'il comporte.

Les auteurs qui ont traité de l'aménagement des forêts ont signalé les différents points sur lesquels il convient de porter son attention, pour que l'on puisse 
apprécier, en connaissance de cause, les améliorations et les réformes dont le traitement d'une forêt est susceptible. Ces points sont au nombre de trente et un dans l'ouvrage de M. Salomon, ancien directeur de l'école forestière de Nancy. Les voici dans l'ordre que cet auteur a suivi :

$1^{\circ}$ Nom du bois ou de la forêt; $2^{\circ}$ origine; $3^{\circ}$ position géographique; $4^{\circ}$ orientement; $5^{\circ}$ situation et exposition; $6^{\circ}$ climat; $7^{\circ}$ vents; $8^{\circ}$ étendue; $9^{\circ}$ nature du sol; $10^{\circ}$ essences et nature du bois; $11^{\circ}$ places vides et clairières; $12^{\circ}$ consistance actuelle de la forêt; $13^{\circ}$ maladies, insectes; $14^{\circ}$ surveillance et délits; $15^{\circ}$ minéraux et carrières; $16^{\circ}$ chasse et bêtes fauves; $17^{\circ}$ routes et chemins; $18^{\circ}$ limites; $19^{\circ}$ fossés; $20^{\circ}$ bornes; $21^{\circ}$ rivières, canaux, ruisseaux; $22^{\circ}$ pêche; $23^{\circ}$ maisons; $24^{\circ}$ enclaves; $25^{\circ}$ pấturage; $26^{\circ}$ droits d'usage; $27^{\circ}$ établissements et lieux de consommation; $28^{\circ}$ disfances approximatives; $29^{\circ}$ produits moyens des dix dernières années; $30^{\circ}$ débit des bois et marchandises usitées; $31^{\circ}$ prix courant de ces marchandises.

Cette nomenclature comprend à peu près tout ce qu'il est essentiel de connaître quand on veut aménager une forêt. Son principal défaut, selon moi, c'est que les matières n'y sont pas classées d'une façon méthodique qui soit propre à les fixer dans la mémoire.

Les divers objets qui sont de nature à exercer ụne influence sur l'aménagement d'une forêt peuvent se partager en cinq groupes bien caractérisés, et rela- 
tifs : le premier, à la forêt considérée dans les éléments qui la constituent ou qu'elle contient; le deuxième, à sa conservation et à son entretien; le troisième, aux dépenses qu'elle occasionne; le quatrième, à son exploitation et à ses produits; le cinquième, aux débouchés dont elle jouit.

Si nous adoptons celte classification, nous rangerons :

Dans le premier groupe, le nom, l'origine, la positi on géograpliqque et administrative, les limites, les tenants, la contenance générale, la contenance du sol boisé ou susceptible de le devenir, les enclaves, les vides et clairières, les lacs, étangs ou marais, les cours d'eau naturels ou artificiels, navigables ou flottables, les rigoles et les fossés d'assainissement, les routes et chemins, et autres moyens de vidange, les maisons forestières, les scieries, les constructions diverses, la configuration et "la nature du sol, les mines et carrières, le climat, le peuplement, les pépinières, le règne animạl ${ }^{1}$;

Dans le deuxième, les dommages auxquels la forêt est exposée de la part des animaux et insectes, du feu, des météores, des délits; la surveillance, l'entretien des voies de transport;

Dans le troisième, le prix des travaux dans la locaité, les dépenses afférentes à l'exploitation, à la sur-

1. On remarquera sans doute que ce groupe contient un trèsgrand nombre d'objets et que sa subdivision serait fort désirable. J'ai cherché une base pour cette subdivision, sans réussir à en trouver une claire, logique, rationnelle. (Note de la ze édition.) 
veillance, à la réparation des voies de transport, à l'entretien des maisons, scieries, etc., etc., des pépinières, des plantations, des fossés d'assainissement, des limites, etc., etc.;

Dans le quatrième, l'exposé de l'aménagement en vigueur, le débit et le prix des bois, les produits en nature, les produits en argent, les produits accessoires, les produits immatériels ou indirects;

Dans le cinquième, enfin, les droits d'usage, les servitudes d'intérêt public, les lieux de consommation, le prix des bois aux lieux de consommation, la différence entre ce prix et celui des bois en forêt.

Il est nécessaire d'étudier tous ces points avec le plus grand soin, de les bien définir. Ce travail est certainement un de ceux qui, dans l'aménagement d'une forêt, exigent le plus d'esprit d'observation, de netteté dans le coup d'œil, de tact, de jugement et de sagacité. Il est destiné à former la base de l'édifice qu'on se propose de construire, ou plutôt l'arsenal dans lequel on devra puiser tous les matériaux nécessaires. A l'aide d'une bonne statistique, on peut, sans aller sur le terrain, régler la marche des exploitations.

Parmi les articles compris dans la statistique, il y en a beaucoup qu'il suffit de traiter d'une manière générale; il y en a quelques-uns, au contraire, qui doivent être l'objet d'un examęn très-détaillé. Pour éviter la confusion que cet examen occasionnerait, si on en consignait les résultats au milieu des généralités, il est d'usage d'en faire la matière d'un docu- 
ment spécial. La statistique comprend donc deux sortes de renseignements : les renseignèments généraux, les renseignemenis spéciaux.

Occupons-nous d'abor'd des renseignements généraux. Je vais passer en revue tous les points énumérés ci-dessus; $j$ 'en trouverai un grand nombre qu'il aura suffi d'énoncer pour faire comprendre les recherches qu'ils réclament; mais il y en a sur lesquels j'aurai à donner des explications. 


\title{
CHAPITRE DEUXIEME.
}

\author{
REXSEIGNEMENTS GÉXERAUX.
}

ARTICLE PREMIER.

PLAS DE LA FORËT.

Lorsque l'on entreprend la statistique d'une forêt, le premier document qu'il y a liẹu de se procurer est un plan exact de cette forêt. S'il n'existe pas, il faut le dresser; y figurer, au moyen : de courbes, de hachures ou de teintes, les principaux mouvements du terrain; y rapporter les enclaves, jardins, étangs et tous les emplacements quelconques qui seraient affectés à une autre destination qu'à la production du bois; y tracer les routes, chemins, cours d'eal, et généralement toutes les lignes séparatives, naturelles ou artificielles, entre les parties de la forêt qui seraient placées dans des conditions toutes particulières, au double point de vue de la situation et de l'exposition; donner à ces partiés de la forêt que l'on désigne communément par le nom générique de canton des noms spéciaux, si elles n'en ont déjà. 
Ce plan est nécessaire pour l'intelligence du cahier descriptif, dans lequel on consigne les renseignements dont je vais refaire l'énumération raisonnée.

\section{ARTICLE II.}

ÉTAT DE LA FORÊT CONSIDÉRÉE DANS LES ÉLÉMENTS QUi LA CONSTITUENT OU QU'ELLE RENFERME.

$1^{\circ}$ Nom et origine.

$2^{\circ}$ Posilion géographique el administrative.

Ces renseignements, destinés à constater l'identité de la forêt, ne réclament pas d'observation.

$3^{\circ}$ Limites. - Faire connaître la nature et l'état des limites, fossés, bornes, etc.

$4^{\circ}$ Tenants. - Indiquer la nature des propriétés qui confinent à̀la forêt.

$5^{\circ}$ Contenance générale et par canton. - Il n'est pas indispensable, lorsqu'on aménage une forêt, d'en connaître la contenance d'une manière parfaitement. exacte. Quelques hectares de plus ou de moins sont de peu d'importance, surtout dans les futaies dont la possibilité est en partie basée sur le volume. La triangulation préalable de la forêt n'est donc point d'une rigoureuse nécessité; mais il faut absolument que les limites en soient assurées, et par conséquent que la délimitation et le bornage en aient été effectués. S'ils ne l'avaient pas été, il y aurait lieu d'y procéder avant de s'occuper de l'aménagement proprement dit. 
$6^{\circ}$ Contenance du sol boisé ou susceptible de le devenir. - Donner ce renseignement, pour la totalité de la forêt d'abord, et ensuite pour chaque canton.

$7^{\circ}$ Enclaves. - Faire connaître leur étendue, leur culture, la catégorie de propriétaires à laquelle elles appartiennent, les inconvénients qu'elles pourraient présenter pour la conservation du sol forestier.

$8^{\circ}$ Vides et clairières. - En indiquer la contenance pour chaque canton, et spécifier les parties qui seraient susceptibles de reboisement et celles qui ne le seraient pas.

$9^{\circ}$ Lacs, étangs ou marais. - En indiquer éga- lement la contenance par canton, et s'expliquer sur la possibilité et l'utilité de leur desséchement.

$10^{\circ}$ Cours d'eau. - Faire connaître leur nom, leur direction, leur développement, leur utilité pour la vidange et le débit des bois, en considérant séparément les cours d'eau flottables et navigables, les cours d'eau seulement flottables, les cours d'eau non flottables, mais susceptibles de le devenir, les cours d'eau non floltables et devant rester tels.

$11^{\circ}$ Rigoles et fossés d'assainissement. - Quel est leur développement, leur état, leur efficacité?

$12^{\circ}$ Routes et chemins, et moyens de vidange établis sur le sol forestier. - Faire connaître leur nom, leur nature, leur développement en longueur et en largeur, leur étát d'entretien, et généralement tout 
ce qui est nécessaire pour que l'on puisse apprécier ultérieurement lès améliorations dont ils devraient être' l'objet.

$13^{\circ}$ Routès et chemins établis sur le domaine public. - Faire connaître leur nom, leur étendue, leur développement, leur utilité pour la vidange des produits de la forêt, en considérant séparément les routes impériales, les routes départementales, les chemins de grande communication, les chemins vicinaux, les chemins imposés par une servilude.

$14^{\circ}$ Maisons forestières. - Quel est leur nombre, leur situation, leur état de conservation, la place qu'elles occupent avec les jardins qui en dépendent.

$15^{\circ}$ Scieries. - Donner les mêmes renseignements, en distinguant les scieries qui appartiennent à l'État de celles qui appartiennent à des particuliers. Donner, en outre, tous les renseignements désirables sur le mécanisme de ces usines, èt la quantité de planches qu'elles sont susceptibles de débiter.

$16^{\circ}$ Constructions diverses. - Les spécifier, indiquer la place qu'elles occupent et s'expliquer sur leurs avantages ou leurs inconvénients.

$17^{\circ}$ Configuration du sol. - Cet article est trèsimportant au double point de vue de la végétation et de la vidange. On le traitera donc de manière à donner une idée aussi exacte que possible des principaux mouvements.du terrain et des pentes qu'il présente. On fera connaître la hiauteur au-dessus du 
niveau de la mer des points culminants, et les aspects généraus de la forêt.

$18^{\circ}$ Nature du sol. - Je ne pourrais, sans entre dans le domaine de la culture, exposer ici unethéorie complète sur la classification des sols forestiers. Je me bornerai à faire saisir les difficultés de cette classification, et à indiquer la méthode d'investigation qui me parait la plus propre à atteindre le but que l'on poursuit, lorsque l'on s'occupe de recueillir ces renseignements si importants de la statistique.

Montrons d'abord qu'une classification des sols suivant la qualité, c'est-à-dire la fertilité, classification très-difficile dans l'agriculture proprement dite, est impossible en sylviculture, et que la prétention de distinguer, dans les terrains de diverses natures qui peuvent exister dans une forêt, ce qui est excellent de ce qui est bon, ce qui est bon de ce qui est médiocre, ce qui est médiocre de ce qui est mauvais, ne saurait aboutir à aucune donnée précise.

Pour qu'il en fùt autrement, il faudrait qu'il y eût dans la composition chimique ou dans les propriétés physiques des sols, des éléments, des caractères qui pussent être considérés comme des signes incontestables d'un certain degré de fertilité. Il faudrait qu'on pût dire : suivant qu'un terrain contiendra plus ou moins de substances de telle ou telle nature, il sera susceptible de produire plus ou moins de bois de telle ou telle qualité; or, c'est là ce qu'on ne saurait apprécier : chacun connaît le rôle important que 
les sels et les alcalis jouent dans la culture arable comme substances nutritives. Dans la culture forestière, ce rôle est très-limité; il ne paraît pas du .moins, d'après les expériences faites jusqu'à ce jour, qu'il y ait lieu de s'en préoccuper sérieusement. Personne n'ignore l'excellent effet des substances animales sur la végétation des plantes agricoles. En sylviculture, cet effet est nul, s'il n'est nuisible. Enfin, c'est un fait parfaitement établi que les céréales, suivant que le terrain est argileux, calcaire ou siliceux, donnent des récoltes bien différentes. En sylviculture, si le chêne prospère surtout dans les terrains argileux, on le voit aussi en fort bon état de croissance dans des sables presque purs; si le hêtre préfère les terrains calcaires, il acquiert souvent dans les terrains siliceux de fort belles dimensions.

Il est certain que les propriétés physiques des terres, la cohésion, l'aptitude au desséchement, l'hygroscopicité, ont cependant une grande influence sur la végétation des bois; mais ces propriétés sont suseeptibles d'être modifiées par une foule de circonstances : par la situation, l'exposition, le climat, et un sol trop compactc pour que le bois y prospère dans un pays plat, deviendra fertile dans un pays en pente. Un sol qui serait trop léger, trop perméable à une certaine altitude, perdrait cet inconvénient à une altitude plus grande, etc., etc.

A quelque point de vue qu'on se place, au point de vue chimique comme au point de vue physique, lés qualités des sols semblent donc se soustraire à 
toute classification rigoureuse, et nous ne conserverons pas le moindre doute à cet égard, si nous considérons l'influence qu'exerce sur la fertilité des terrains forestiers un élément dont je n'ai pas encore parlé, mais que tout le monde devine : l'humus:

L'humus, résultat direct de la végétation, restitue au terrain les substances minérales que celle-ci lui a empruntées, l'enrichit de matières carboniques, et, par ses propriétés physiques, le rend propre à s'assimiler dans les proportions les plus convenables l'air, l'eau, la chaleur, ces trois agents qui sont la source de la vie végétale.

L'action de l'humus est donc prépondérante dans la fonction des sols forestiers; mais alors on ne saurait songer à établir des degrés. de fertilité pour ces sols, d'après les éléments qui les constitueraient au moment de l'observation, puisqu'ils sont destinés à être constanment et puissamment modifiés par l'effet même de la végétation.

L'enseignement à tirer de ce qui précède, c'est que ce que l'on a de mieux à faire, quand on arrive à la partie de la statistique relative au sol, est d'indiquer la nature de la base minéralogique, la profondeur de la terre végétale, les éléments qui y dominent, celle des catégories de terrains décrites dans le Cours de culture de MM. Lorentz et Parade, à laquelle il appartient.

Ces renseignements, rapprochés de ceux donnés à l'article Climat, mettront à même de juger autant que cela se peut, quand on n'a pas vu les lieux, de 
la puissance productive du sol et des conditions à réaliser pour qu'elle soit portée à son maximum ${ }^{1}$.

$19^{\circ}$ Mines et carrières. - Faire connaître leur

1. Hundeshagen a fait une classification des sols au point de vue forestier.

Il range :

Dans la $1^{\text {re }}$ classe (les sols très-riches), toutes les formations calcaires en général. - Parmi elles, le tuf calcaire, par sa facile décomposition, forme d'ordinaire les plus fertiles; - les différentes couches secondaires de gypse et de marne de diverses natures; les formations volcaniques (laves), celles de basalte, de trapp et des brèches trappéennés; l'euphotide, le chlorite ou grès flexible, la serpentine, le schiste magnésien et le schiste argileux, lorsqu'il contient également de la magnésie; la marne oolithique, lorsqu'elle a plus de 10 pour 100 de chaux; les gisements quartzocalcaires de quelques couches de grès, lorsqu'ils forment un sol calcaire ferrugineux; les porphyres.

Les sols de cette classe peuvent faire croître, même sans mélange d'humus et d'engrais, les essences les plus exigeantes, ou du moins les empêcher de dépérir. On n'y trouve jamais de fougères, de bruyères ni de genêts.

Dans la $2^{\mathrm{e}}$ classe (de fécondité moyenne), les schistes argileux, abondants en quartz et pauvres en clraux, en magnésie et en oxyde de fer; les granites et gneiss; les schistes siliceux; le tuf quartzeux et ordinaire; le schiste micacé; le grès primitif; les variétés les plus riches en argile du grès bigarré et du grès oolithique.

Pour que les essences qui exigent un bon sol viennent bien dans ces terrains, il faut qu'ils contiennent de l'humus en suffisante quantité. On y trouve le genêt, la bruvère, la myrtile.

Dans la $3^{e}$ classe (sols pauvres), les grès bigarrés en général; les grès de nouvelle formation reposant sur la chaux coquillière, les grès du Keuper et du Lias; les brèches; les molasses et, en général, les grès de la plus nouvelle formation. Il faut à ces terrains beaucoup d'humus; le hêtre, le charme, le tilleul, le sapin, le pin du Nord ne s'y conservent que par des soins et un traitement convenables. Le frène, l'aune, l'érable, etc., n'y viennent 
nature, leur importance, les difficultés de leur exploitation.

$20^{\circ}$ Climat. - Le mot climat, dans son acception générale embrasse toutes les modifications dont. l'atmosphère est susceptible, eu égard à la température, à l'humidité et aux courants qui s'y agitent ${ }^{1}$.

Ces modifications, qui sont en quelque sorte solidaires, et qui s'engendrent les unes les autres, dérivent d'une cause.première et unique, la chaleur, et se traduisent par des effets divers, dont le plus manifeste, le plus influent et le plus facile à constater, est l'abaissement ou l'élévation de la température. Aussi est-ce d'après l'état de cette dernière sur certains points de notre planẹte, qu'ont été déterminés les différents climats.

La terre a une chaleur propre et centrale, mais l'influence que cette chaleur exerce sur la température de la surface est insignifiante, et l'on peut en conséquence regarder le soleil comme la source exclusive de la chaleur.

L'action du soleil est modifiée, on le sait, par un grand nombre de circonstances dont les principales sont :

La latilude. La différence de température moyenne

plus originairement. On y trouve en quantité les fougères, les genêts, les bruyères.

Dans la 4e classe (sols très-maigres), les terrains formés de cailloux roulés, de galets et de sable mouvant.

1. Cours de cullure des bois, page 3. 
est, d'après M. de Humboldt, de 0,62 par degré de latitude.

L'alıitude. Sous l'équateur, à une élévation de 3,000 mètres, on jouit de la même température moyenne qu'à Montpellier ou à Madrid.

L'état de la surface. Dan's le désert du Sahara, les sables s'échauffent au point de faire monter le thermomètre à 50 ou $60^{\circ}$. Dans les forêts de l'Orénoque il y a des vides couverts de rochers qui, la nuit, ont une température plus élevée de $11^{\circ}$ que l'air ambiant. On sait enfin que les grandes masses d'eau s'échauffent ou se refroidissent lentement, et que le climat des terres voisines s'en ressent.

Telles sont les causes principales qui influent sul' la répartition de la chaleur à la surface du globe, et qui déterminent le climal moyen général d'une contrée; mais l'état de l'atmosphère est en outre modifié secondairement et localement par d'autres éléments, le vent et l'eau, dont l'action est variable à l'infini, suivant la configuration du sol.

Froids ou chauds, secs ou humides, suivant les régions qu'ils ont traversées, les vents peuvent, d'après les expériences de M. de Gasparin, abaisser de $6^{\circ}$ la température produite par la chaleur directe, et de beaucoup plus, celle produite par la chaleur réfléchie du soleil.

Les brouillards obscurcissent l'atmosphère et empềchent l'action bienfaisante de la lumière.

Les nuages abaissent la température en été parce qu'ils interceptent les rayons lumineux, et l'élèvent 
en hiver parce qu'ils s'opposent au rayonnement nocturne.

La pluie, au moment où elle se forme, a pour effet d'élever la température de l'air ambiant. La neige empêche les corps qu'elle recouvre, de se refroidir par le rayonnement.

Le climat se soustrait donc, comme le sol, à foute classification rigoureuse, parce qu'il est susceptible de se modifier, pour ainsi dire, à chaque accident de terrain; et si l'on se bornait dans la statistique à définir le climat d'une forèt, d'après la température moyenne de la contrée dans laquelle elle serait située, on ne donnerait pas les indications suffisantes pour le faire apprécier ${ }^{1}$.

1. M. Charles Martins a partagé la France en cinq climats; il range :

Dans le premier (climat excessif), sous le nom de climat vosgien, toute la contrée comprise entre le Rhin, la Còle-d'Or, les sources de la Saone, la chaîne de montagnes qui va de Mézières à Auxerre. La température moyenne de ce climat est, pour l'biver, dé $0^{\circ}, 6$; pour l'été, de $18^{\circ}, 6$; pour l'année, de $9^{\circ}, 6$.

Dans le deuxième (climat maritime), sous le nom de climat séquanien, toute la contrée comprise éntre la frontière du nord, depuis Mézières jusqu’à la mer, le contre-fort du plateau qui règne de Mézières à Auxerre et le cours de la Loire et du Cher. La température moyenne de ce climat est, pour l'hiver, de $3^{\circ}, 95$; pour l'élé, de $17^{\circ}, 6$; pour l'année, de $10^{\circ}, 9$.

Dans le troisième (climat tempéré), sous le nom de climat girondin, la contrée comprise entre la Loire et: le Cher jusqu'aux Pyrénées, puis à travers le plateau central de l'Auvergne. La température moyenne de ce climat est, pour l'hiver, de $5^{\circ}$; celle de l'été, de $20^{\circ}, 6$; celle de l'année, de 190,7 .

Dans le quatrième (climat également tempéré), sous le nom de 
Je pense dès lors que, dans l'étude du climat d'une forêt, il convient de prendre pour guide et. d'adopter comme circonstance caractéristique, la nature et l'état de végétation des plantes qui croissent spontanément dans la région où est située cette forêt ${ }^{1}$.

climat rhodanien, la contrée comprise entre la vallée de la Saône et du Rhonne, depuis Dijon et Besançon jusqu'à Viviers, le massif des liautes Alpes, une partie des basses Alpes. La température moyenne de ce elimat est, pour l'hiver, de $2^{\circ}, 3$; pour l'été, de $21^{\circ}, 3$; pour l'année, de $11^{\circ}$.

Dans le cinquième, enfin (climat cliaud), sous le nom de climat méditerranéen, le surplus de la France, La température moyenne de ee' climat est, pour l'hiver, de $6^{\circ}, 5$; pour l'été, de $22^{\circ}, 6$; pour l'année, de $14^{\circ}, 8$.

Une pareille classification, bonne pour donner une idée générale des conditions climatériques dans lesquelles se trouve la France, ne saurait être d'aucune utilité pratique sous le rapport agricole et surtout sylvicole. Dins chacune des régions qui y sont comprises, il y a des points plus ou moins élevés au-dessus du niveau de la mer, des expositions différentes et, par conséquent, des rariations de température qui, si elles étaient considérées isolèment, devraient faire ranger les localités où elles se présentent dans un autre climat que celui auquel on les a ratlachées. L'état de la température n'indique pas d'ailleurs quelles sont les espèces susceptibles de croître et de prospérer dans un lieu donné.

1. On désigne ordinairement sous le nom de:

Climat chaud, celui des contrées où eroissent spontanément l'olivier, le figuier, le chêne-liége, le chêne yeuse.

Climat doux, celui des contrées où croissent en plein vent la vigne, l'amandier, le pêclıer, toute espèce d'arbres fruitiers et de plantes potagères.

Climat tempéré, celui des contrées où les arbres fruitiers réussissent bien, et dont les forêts contiennent toutes les essences indigènes, sauf celles du climat chaud.

1. Climat rude, celui des contrées où la culturc des arbres frui- 
Mais ces renseignements serajent encore insuffisants si l'on n'y ajoutait tous ceux que l'on pourra se procurer, sur les variations de la température et les accidents météoriques qui pourraient affecter un lieu donnć.

Indépendamment de l'indication des plantes qui seront cultivées avec succès dans la contrée dont on voudra faire connaitre le climat, on fournira done tous. les renseignements possibles sur les écarts de température, la fréquence, l'intensité et la persistance des vents, de la pluie, des brouillards, de la gelée, de la neige, de la grêle, du verglas, 'du givre.

$24^{\circ}$ Nature et élat du peuplement. - Décrire à grands traits le peuplement, et par conséquent faire connaitre les essences dont il se compose, son état de consistance et de végétation suivant les sols et les climats, l'étendue occupée par le taillis, par les futaies. Donner pour les principales essences, tous les renseignements qu'on pourra se procurer sur leur croissance, leur longévité, la facilité plus ou moins grande avec laquelle elles sont susceptibles de se régénérer, soit par les semences, soit par les souches, l'âge auquel elles sont présumées devoir atteindre

tiers et des plantes potagères délicates est difficile, et dont les forêts sont peuplées principalement d'essences résineuses.

Climat très-rude, celui des contrées où la culture du sarrasin, de l'orge et des pommez dè terre est la seule possible, et dont les 'orêts contiennent comme essences dominantes, l'épicéa, le mélèze, le hêtre, celui-ci mal venant. 
l'époque de leur plus grand accroissement moyen.

$22^{\circ}$ Pépinières. - Indiquer leur emplacement, leur étendue, les essences qu'on y cultive, leur état d'entretien, les ressources qu'elles offrent pour le repeuplement.

$23^{\circ}$ Règne animal. - Dire quelles sont les espèces d'animaux qui peuplent la forêt et les cours d'eau; si elles y sont abondantes et utiles.

ARTIGLE III.

CONSERVATION ET ENTRETIEN.

$24^{\circ}$ Insectes et animaux nuisibles. - Quels sont les insectes et les animaux qui attaquent les bois, les désordres qu'ils causent, les mesures à prendre pour les détruire?

Le gibier, le lapin principalement, cause de trèsgrands dommages dans certaines forêts; il importe donc de ne pas oublier cet article quand on s'occupe des circonstances qui sont susceptibles d'entraver la végétation.

$25^{\circ}$ Incendies. - Faire connaître si les incendies sont fréquents, quelles en sont les causes, comment on pourrait en empêcher le retour.

$26^{\circ}$ Délits. - Dire le nombre, la nature, les causes; les conséquences, l'importance des délits, le nombre et la profession des délinquants solvables ou insolvables. 
$27^{\circ}$ Surveillance. - Quel est le nombre des brigadiers et gardes, leur salaire; quels sont le nombre et l'étendue des triages; quels sont les circonstances qui favorisent ou entravent la surveillance? Servitudes, constructions à distance prohibée, etc., etc.

$25^{\circ}$ Entretien des routes el chemins. - Quel est le nombre des cantonniers, leur'salaire, le nombre de kilomètres que-chacun d'eux est chargé d'entretenir; quelles sont les circonstances qui exercent une influence sur l'accomplissement de leur' tàche?

\section{ARTICLE IV.}

DËPENSRS.

$29^{\circ}$ Prix des travaux dans la localilé. - Faire' connaître le prix de la main-d'œuvre, suivant la nature du travail, le prix de location d'une bête de charge, d'une voiture attelée.

$30^{\circ}$ Dépenses occasionnées par la surveillance.

$31^{\circ}$ Dépenses occasionnées par l'entretien des chemins forestiers.

$32^{\circ}$ Subrentions pour la réparation des chemins vicinaux.

$33^{\circ}$ Dépenses occasionnées par l'entretien des maisons forestières, des scieries el autres constructions.

$34^{\circ}$ Crédlit affecté moyennement chaque année à l'entretien des pépinières, aux repeuplements artificiels, 
aux assainissements, auix exploitations par économie. - $35^{\circ}$ Idem, pour le curage des fossés de limite, la réparation des bornes, etc., etc.

$36^{\circ}$ Impositions, dépenses diverses. - Les renseignements que comportent les articles précédents se devinent, sans qu'on ait besoin de les signaler.

ARTICLE V.

EXPLOITATION ET PRODEITS.

$37^{\circ}$ Exposé de l'aménagement en vigueur.

$38^{\circ}$ Débit des bois. - Faire connaître à quel usage on emploie les principales essences, et surtout les ressources qu'elles pourraient présenter pour les constructions navales.

Établir autant que possible, pour chacune de ces essences, étant donnée sa grosseur à un mètre du sol, le rapport existant :

Entre le volume conique et le volume réel de la tige,

Entre le volume conique de la tige et le volume réel total,

Entre le volume réel de la tigẹ et le volume de la tige propre à l'œuvre,

Entre le volume réel total et le volume propre à l'œuvre,

Entre le volume réel total et le volume propre au chauffage: 
Entre le volume réel total et le volume propre ąu charbon,

Entre le volume réel total et le nombre des bourrées,

Entre le mètre cube de bois brut et l'unité des marchandises fabriquées en forêt. On saura de cetle manière combien il faut de bois pour fabriquer une grosse de sabots, un cent de merrain, un stère de chauffage, etc.,

Entre le mètre cube de bois brut et le' volume de la quantité de marchandises qu'on peut en tirer.

39. Prix délaillé des bois. - Faire connaître le prix sur pied du mètre cube de bois, suivant la destination à laquelle il est propre, œuvre, chauffage ou charbon; les variations que ce prix a subies depuis un certain nombre d'années, la plus-value donnée au bois par la façon dont il est l'objet en forêt, ou, en d'autres termes, la dillérence entre le prix du mètre cube en grume et sur pied, et le prix de la quantité de marchandises qu'il est susceptible de fournir.

$40^{\circ}$ Produils en nalure des bois. - Faire connaître le volume exploité chaque année, dans les futaies, dans les taillis, en coupes principales, en coupes intermédiaires par contenance et par volume.

Établir la part de ce volume propre à 'l'œurre, au chauffage et au charbon.

$41^{\circ}$ Produits en argent des bois. - Faire connaitre la valeur en argent des produits en bois ci-dessus spécifiés.

$42^{\circ}$ Produits en argent, accessoires. - Faire 
connaître ce qu'on retire des mines et carrières, de la chasse, de la pêche, de la glandée, de la faînée, des pépinières, des amendes, des indemnités pour bris de. réserve et tous autres menus produits.

$43^{\circ}$ Produits immatériels ou indirects. - Les produits immatériels d'une forêt sont sans intérêt pour le propriétaire, quand ce propriétaire est un particulier; mais quand ce propriétaire est l'État, ils ont, au contraire, la plus haute importance. Les 30 à 40 millions que les forêts nationales rapportent chaque année au Trésor sont assurément fort peu de chose à côté des ressources incalculables qu'elles offrent à l'industrie, et des avantages qu'elles présentent pour la défense du territoire, la conservation du climat et du sol, le régime des eaux. Il convient donc de faire connaître, dans la statistique d'une forêt, l'influence que cette forêt pourrait exercer sur la conservation du climat et du sol, le régime des eaux, l'existence ou le bien-être des populations environnantes.

Le nombre, la profession et le salaire des ouvriers employés à l'exploitation, au débit, à la vidange, aux améliorations diverses, donneront une idée de l'importance de la forêt sous ce dernier rapport.

\section{ARTIGLE VI.}

DÉ B O U C II ÉS.

$44^{\circ}$ Droits d'usage. - Exposer la nature et l'étendue des droits d'usage, l'influence qu'ils ont sur 
l'exploitation de la forêt. Si cette influence était fàcheuse et qu'il fùt utile de procéder à l'extinction des droits, il faudrait provoquer immédiatement cette opération et surseoir à l'aménagement proprement dit.

$45^{\circ}$ Servitudes d'intérét public. - Ces servitudes doivent être nécessairement prises en considération dans l'étude du plan d'exploitation de la forêt à aménager. Il convient donc de les faire connaitre.

$46^{\circ}$ Lieux de consommalion. - Indiquer les principaux lieux de consommation, et notamment les usines, la distance qui les sépare de la forêt, la nature et la quantité des bois qui s'y consomment.

$47^{\circ}$ Prix des bois aux lieux de consommation. Ce 'renseignement est essentiel, car, en le rapprochant du prix des bois en forêt, on pourra se faire une idée exacte de la facilité ou de la difficulté des. transports. 


\title{
CHAPITRE TROISIEMUE
}

\author{
RENSEIGNEMENTS SPECIAUX.
}

\section{ARTIGLE PREMIER.}

POINTS A EXAMINER.

Après avoir étudié une forêt dans son ensemble, eu égard seulement aux grandes divisions ou cantons dont elle se compose, il est nécessaire de l'étudier dans ses détails, et de décrire pour chacune de ces divisions ou cantons, toutes les parties caractérisées par l'une des particularités qui sont de nature à influer sur le traitement applicable à un massif.

Ces particularités sont :

$1^{\circ} L^{\prime}$ age des bois. - Il est évident que c'est presque toujours la circonstance déterminante, dans la fixation de l'époque à laquelle un massif doit être régénéré.

$2^{\circ}$ La nature des essences. - Des bouleaux ne sauraient être exploités au même àge que des chênes ou des hêtres. Des résineux ne peuvent pas être soumis aux mêmes traitements que des bois feuillus.

$3^{\circ}$ L'état de la végétation. - Cet état permet de 
reculer plus ou moins le móment de la régénération.

$4^{\circ}$ La qualité du sol. - Selon que le sol sera substantiel ou maigre, profond ou non, perméable ou imperméable, un massif pourra être conduit jusqu'à un âge plus ou moins avancé, et sera plus ou moins susceptible de se régénérer et de se perpétuer par les souches.

$5^{\circ}$ L'exposition et la situation. - $\mathrm{A}$ l'exposition du nord, la végétation et les dangers qui la menacent sont bien différents qu'à celle du midi. L'exposition doit donc être prise en considération dans le choix du traitement; la situation ét surtout l'altitude doivent l'ètre également, car elles n'exercent pas moins d'influence sur la croissance des bois.

Il est certain que chacune des circonstances que je viens d'énumérer doit être soigneusement examinée, lorsqu'on veut régler d'une manière rationnelle le traitement d'une forêt.

Pour assurer cet examen, voici comme on procède.

\section{ARTICLE II.}

DC PA AELLAIRE.

On commence par diviser chaque canton en autant de parties qu'il y existe de peuplements différents par l'âge; cela fait, si ces parties renferment des parties dissemblables sous le rapport des essences, de l'état de la végétation, de la nature du sol, de l'expo- 
sition ou de la situation; on les subdivise en autant de parcelles qu'il y a d'essences, d'états de végétation, de qualités de sol, d'expositions et de situations particulières.

Telle est l'opération connue sous le nom de parcellaire. C'est une des plus importantes de l'aménagement, comme nous le verrons plus tard, et comme il est facile d'ailleurs de le comprendre dès à présent.

Le parcellaire est en effet indispensable pour qu'on puisse circonscrire les coupes, dresser le plan d'exploitation; parce que seul il permet de rapprocher, de grouper les peuplements qu'il convient de régénérer à la même époque et par le même mode.

Le parcellaire ne saurait donc être fait avec trop d'attention, et son élément, la parcelle, peut se définir, d'après les explications que je viens de donner : une portion de forêt homogène, quant à l'áge, à l'essence et aux conditions de végétation, et par conséquent, dont toutes les parties constituantes sont susceptibles d'être soumises au même traitement.

Cette définition, que je n'invente pas, que j'emprunte à la tradition, est fort claire et me dispenserait d'entrer dans de nouvelles explications, si les diversités d'état que présente une forêt, étaient jamais bien trańchées, bien évidentes; si l'homogénéité parfaite dans un peuplement ne se renfermait pas, presque toujours, dans des limites extrêmement étroites. Malheureusement nos forêts sont, en général, fort irrégulières; et si on voulait y séparer les unes des autres, en prenant et en appliquant à la lettre la défi- 
nition de la parcelle, toutes les parties dissemblables par un des caractères que j'ai signalés, on arriverait à y former presque autant de parcelles qu'il y aurait d'arbires, ce qui enlèverait évidemment au parcellaire toute utilité.

Il faut ici chercher une règle qui empêche que le parcellaire ne dégénère en une opération trop minutieuse; or, celte règle est indiquée par le but que l'on veut alleindre. Quel est ce but? - Nous le savons déjà, c'est la connaissance et la réunion de tous les peuplements susceptibles d'être régénérés à la même époque et par le même mode; mais la culture nous enseigne qu'il y a, pour l'exploitation des bois, deux méthodes principales : la méthode du jardinage, qui consiste à prendre chaque année çà et là, sans s'astreindre à aucune limite de contenance, les arbres les plus âgés; la méthode des coupes régulières et de proche en proche, dont une des exigences est, au contraire, la concentration de la coupe annuelle dans une circonscription aussi étroite que possible. Nous n'avons pas à nous oćcuper de la première de ces méthodes, car elle est inconciliable avec l'aménagement pris dans son acception scientifique; et 'si nous interrogeons la portée de la seconde, nous reconnaîtrons que -puisqu'elle exige que chaque année l'exploitation soit concentrée dans, une certaine circonscription, la contenance la plus petite que l'on puisse donner à une parcelle, est celle au-dessous de laquelle l'étendue d'une coupe annuelle ne saurait descendre sans inconvénients palpables, étendue 
variable, du reste, suivant.les localités, selon le propriétaire.

Quelle que soit, par conséquent, l'irrégularité d'un peuplement, on doit considérer comme l'élément irréductible du parcellaire une portion de ce peuplement, égale au moins en étendue à une coupe annuelle; y ajouter le peuplement environnant, pour le confondre dans la même parcelle, s'il participe de la même irrégularité; le séparer dans le cas contraire, pour en faire une parcelle distincte, si son éterídue est d'ailleurs assez grande ${ }^{1}$.

Nous tirerons une autre conséquence du même principe : c'est que dans le tracé des lignes séparatives des parcelles, il n'est pas nécessaire de s'astreindre à suivre tous les contours, toutes les sinuosités indiquées par les changements d'état du peuplement, et qu'il faut, au contraire, redresser ces lignes toutes les fois 'qu'il s'agit de ne transporter, d'une parcelle dans la parcelle voisine; que des portions peu étendues.

Quant à la limite supérieure pour la contenance des parcelles, il n'y en a rigoureusement pas; en

1. On m'a fait remarquer qu'au moment où on procède au parcellaire, on ne connaît pas encore l'étendue de la coupe annuelle. Cela est vrai puisqu'on ne connaît pas la possibilité, mais on sait déjà quelle est l'importance qu'il convient de donner à une coupe pour 'qu'elle puisse se vendre avantageusement et dans des conditions qui en favorisent la régénération. Au reste, je conviens que dans les futaies surlout il est impossible de prévoir exactement quelle sera l'étendue de la coupe annuelle, 'mais il s'agit iei d'une approximation. (Note de la $2^{e}$ édition.) 
sorte que, si une forêt était, par exemple, située èn plaine, composée de bois de même âge ou régulièremẹnt entremêlés, tout à fait homogène, enfin, quant aux conditions de peuplement, de sol, de climat, de situation, son parcellaire deviendrait inutile, la marche des coupes pouvant indifféremment avoil lieu dans un sens ou dans l'autre.

Ainsi, voilà qui est bien convenu : Quelles que soient les diversités d'état qui existent dans une forêt, il n'y a pas à en tenir compte, lorsque leur expression superficielle, s'il est permis d'employer ces termes, descend au-dessous d'une certaine limite. Voyons maintenant s'il ne convient pas de laisser, en outre, quelque latitude aux aménagistes, en ce qui concerne l'appréciation des dissemblances ellesmêmes, suivant qu'elles sont relatives à l'âge ou aux essences, à la végétation ou au sol, à la situation. ou à l'exposition.

Dissemblances relatives à l'áge. - Puisque l'âge est la circonstance qui sert de base, dans la plupart des cas, pour fixer l'époque de la régénération d'un massif, la différence d'âge doit être prise, avant tout, en considération dans la formation des parcelles. C'est d'ailleurs celle qui attire la première les regards. et qui est la plus évidente; mais s'arrêtera-t-on devant une différence d'un an? - Le simple bon sens répond que non : d'abord, parce que cette différence serait fort difficile à reconnaître; ensuite, parce que ce n'est point un aussi petit écart entre les âges qui pourrait en motiver un entre les époques 
d'exploitation; enfin, parce que dans les futaies, la régénération ne pouvant, en général, avoir lieu sur une place donnée qu'en plusieurs fois, il en résulte nécessairement que sur cette même place il y aura entremêlés des bois qui ne sauraient être aussi exactement âgés les uns que les autres. Il faut s'arrêter, dans le parcellaire, aux différences qui frappent l'œil, et uon à celles qui ne pourraient être consta tées que par le comptage des couches concentriques. Voilà la règle, et par conséquent, dans les futaies on . considère comme étant de même âge des bois entre les âges desquels il n'y a pas un écart de plus de 12 à 15 ans. On adopte, dans les taillis, des limites moins larges, attendu que les différences d'âge y sont ordinairement plus apparentes; nonseulement parce que la croissance des rejets de souches est, quand ils sont jeunes, plus rapide que celle des brins de semis, mais parce qu'il y a nécessairement plus d'homogénéité dans les peuplements dont la régénération remonte à la même époque, leur exploitation ayant lieu, comme on sait à blanc étoc.

Dissemblances relatives aux essences. - Après l'âge, vient l'essence dans l'examen des particularités qui peuvent motiver la formation d'une parcelle. Ici, il ne saurait s'élever des doutes, et toutes les fois qu'un massif renfermera, sur une étendue plus grande que celle qu'il conviendrait de donner à une coupe annuelle, un peuplement dont l'essence dominante dilférera, au point de vue de l'exploitabilité " 
surtout, de celle des bois environnants, on fera de ce peuplement une parcelle distincte.

Dissemb!ances relatives à l'étal de la végétation. - On agira de même qu'il est dit ci-dessus, pour l'état de la végétation, toutes les fois qu'à âge égal, les essences étant les mêmes, cet état sera manifestement assez mauvais, sur une étendue au moins égale à celle d'une coupe, pour que l'on soit obligé d'avancer l'époque de la régénération, d'un nombre d'années plus grand que celui que l'on peut admettre entre les âges des sujets appartenant à la même parcelle.

Dissemblances relatives à la situation et à l'exposition. - Si le partage de la forêt en cantons a élé fait conformément aux principes que j'ai émis, le climat, dans son acception générale, n'exercera aucune influence sur la formation des parcelles; l'exposition ne saurait non plus en avoir beaucoup - quoiqu'elle soit au nombre des principales causes des accidents météoriques qui affectent une contrée - attendu qu'il en aura été tenu compte indirectement dans la subdivision des parcelles, eu égard aux différences de la végétation; mais la situation, en rendant plus ou moins facile l'exploitation et la vidange, a, sous ce double rapport, une action particulière qui motiverait la subdivision d'une parcelle, si elle devait placer des parties de cette parcelle dans des conditions sensiblement différentes.

Dissemblances relatives au sol. - Le sol ne vient qu'en dernier lieu dans la série des circonstances 
que l'on doit apprécier, quand on effectue le par. cellaire d'une forêt; cela s'explique par la difficulté d'en constater la qualité absolue, et par le signe d'après lequel on juge le plus communément de sa qualité relative. Ce signe est l'état de la végétation. Presque toujours, le parcellaire, modifié d'abord d'après la nature des essences, puis d'après l'état de la végétation, n'aura pas besoin de l'être par suite des différences de qualité que pourrait présenter le terrain. Toutefois, puisque la mauvaise qualité du sol n'est pas la seule circonstance qui soit susceptible d'entraver la croissanee des végétaux, il pourrait arriver que des parties de terrain, profondes et substantielles, eussent été comprises dans la même parcelle que des parties maigres et sans profondeur, parce que les premières seraient couvertes d'un peuplement mal venant. Dans ces cas, certainement très-rares; et dont on constalerait l'existence au moyen de quelques sondages, il faudrait évidemment faire des parcelles distinctes des bons et des mauvais terrains.

Le parcellaire est en définitive, comme on peut en juger par ce qui précède, une opération moins compliquée qu'elle ne paraît au premier abord. Lorsqu'il est terminé, on exécute le levé des parcelles qu'on rapporte ensuite sur le plan de la forêt, et on procède, à l'aide de ce plan, à l'examen et à la description détaillés du peuplement et des' conditions dans lesquelles il se trouve. 
ARTICLE III.

DESCRIPTION SPÉCIALE.

Tout n'est pas fini lorsque les parcelles ont été délimitées, levées et rapportées ; il reste à les décrire, $\dot{a}$ en donner une idée aussi complète que possible; car c'est cette description qui décidera de la place qu'elles occuperont dans le plan d'exploitation, de l'époque à laquelle elles devront ètre régénérées, et, en un mot, de la destination qu'on leur donnera.

Après avoir indiqué la contenance d'une parcelle, on fera donc connaître les essences qu'elle renferme, leur âge, leür état de végétation et de consistance, la durée probable du temps pendant lequel elles pourraient rester sur pied sans dépérir. On dira quelle est la nature de la base minéralogique, quelle est la composition de la terre végétale, son élément dominant, sa profondeur, l'état de sa superficie. On dira aussi à quelle altitude approximative la parcelle est située, quelle est son exposition, quels sont les accidents météoriques qu'elle pourrait avoir à redouter, quelle est la voie de vidange par laquelle s'exportent ses produits. Un examen attentif, minutieux, réfléchi, est nécessaire pour que cette description soit de nature à reproduire dans l'esprit du lecteur l'image fidèle des lieux. J'essayerais vainement de donner des règles pour assurer un pareil résultat. 
La manière dont il faut s'y prendre pour apprécier un peuplement, l'aptitude à dégager l'état moyen de ce peuplement de l'espèce de confusion que produisent au premier aspect les variétés d'àge, d'essences, de forme et de consistance qui frappent les yeux, sont des choses que l'on n'enseigne guère. Ce sont presque des dons naturels. Il y a des gens qui ne savent pas voir un peuplement; qui sont par conséquent incapables de le décrire; soit que la dernière impression efface chez eux toutes les autres, en sorte que, s'ils aperçoivent, en achevant la reconnaissance, un bouquet de chênes ou de bouleaux, par exemple, ils croient n'avoir vu que des chênes ou des bouleaux; soit que les nuances qui passent sous leurs yeux ne se fondent pas dans leur esprit, qui fait alors de vains efforts pour les retenir; soit qu'ils ne puissent résumer leur's diverses observations. Ces gens-là ne seront jamais de bons aménagistes. Il y a entre le coup d'œil du forestier et celui du paysagiste une analogie frappante qui me permettra de compléter ma pensée. Tous ceux qui se sont occupés - de dessiner d'après nature ont dù, à leur début, éprouver quelque.embarras à grouper tous les détails. qui se présentaiẹnt à leur regard; à distinguer ce qui était "du premier, du deuxième ou du troisième plan; à masser, pour nous servir de l'expression technique, le feuillage d'un arbre; mais, au bout de quelque temps, les uns y sont parvenus; les autres, au contraire, n'ont jamais pu saisir, dégager les rapports existants entre les parties d'un groupe, et 
en représenter la physionomie générale. Il en est de. même des forestiers; il y en a parfois qui ne savent pas lier leurs impressions et leurs idées, les classer, voir les choses dans leur ensemble, dans leur caractères principaux. Ceux-là sont inhabiles à faire une bonne description; ils se donneront beaucoup de peine pour fixer dans leur mémoire chaque détail, chaque particularité, au fur et à mesure qu'elle se présentera devant eux, et ne pourront tirer aucun profit de leur examen, quand il s'agira de dresser le plan d'exploitation.

ARTICLE IV.

RÉST MÉ.

La reconnaissance spéciale de la forêt exige qu'on en fasse d'abord le parcellaire.

Cette opération consiste à subdiviser chacun des cantons qu'elle comprend, en autant de portions distinctes et séparées qu'il y a de différences bien tranchées dans la composition et les conditions de végétation du peuplement.

Cette subdivision, subordonnée d'abord à l'àge des bois, à la nature des essences et à l'état de la végélation, est modifiée ensuite, s’il y a lieu, conformément aux dissemblances relatives au sol, à la situation, à l'exposition.

Il est impossible de fixer d'une manière précise 
la limite minima au-dessous de laquelle la contenance d'une parcelle ne doit pas descendre; seulement il faut éviter, si la parcelle est couverte de bois, de lui donner une contenance plus petite que celle que devrait avoir une coupe annuelle.

Quand le parcellaire est terminé, on a soin de faire arpenter, lever et rapporter sur un plan toutes les parcelles qu'il a eu pour. résultat d'établir. On donne à ces pàrcelles des signes distinctifs; on les désigne, par exemple, par des lettrés afin de les reconnaître facilement, mais, comme on le verra plus tard, cette désignation n'est que provisoire.

Enfin, on procède à la reconnaissance détaillée de chaque parcelle, reconnaissance qu'on ne saurait faire en même temps que le parcellaire, attendu qu'on a besoin, pour ne pas se tromper dans l'appréciation d'un peuplement, d'en connaître avant tout la contenance, et que ce renseignement n'existe pas encore au moment où le parcellaire s'effectue.

Un cahier spécial, dans lequel sont transcrits les résultats de cette reconnaissance, forme ce qu'on est convenu d'appeler le cahier de la description spéciale.

\section{ARTIGLE V.}

OBSERVATIONS SUR LES PRINCIPES CI-DESSUS DÉVELOPPÉS.

Les idées exposées dans les articles précédents, relativement au parcellaire, ne seront probablement 
pas du goût de tout le monde : on les atlaquera peut- être dans le fond et dans la forme.

Si on consulte les traditions, on y trouvera, en effet, des principes qui diffèrent essentiellement des miens, quoique cependant ils tendent au même but. Au lieu de prendre, comme je le fais, pour élément fondamental du parcellaire, une portion de forêt homogène quant à l'àge, aux essences, aux conditions de végétation; au lieu de subordonner la formation des parcelles aux différences d'état des peuplements qui constituent la forêt, on prend pour élément fondamental du parcellaire une portion de forêt circonscrite par des limites naturelles, et on subordonne en conséquence la formation des parcelles à des circonstances indépendantes de l'état du peuplement. II est vrai qu'on trie ensuite, dans chacune des grandes divisions circonscrites par des limites naturelles, les peuplements homogènes pour en faire des subdivisions; mais ces subdivisions, condamnées d'avance à se fondre dans les divisions dont elles font partie, ne sont appelées à exercer individuellement aucune influence sur la formation du plan d'exploitation.

Tel est le point sur lequel ma manière de voir est surtout en désaccord avec certains errements. II est capital, et je ne peux me dispenser de m'y arrêter.

Si on ne formait les subdivisions dont je viens de parler que pour faciliter l'opération du parcellaire, je ne protesterais pas. On suivrait en réalité la 
marche que j'ai conseillée; mais quand on forme une division avec l'idée préconçue et arrêtée de faire subir la même destinée à la totalité des peuplements qu'elle contient, on met, pour me servir d'une comparaison triviale, la charrue avant les bœufs; et on s'expose évidemment à violer tous les principes de la culture et de l'exploitabilité, en rangeant sous la même loi, en assujettissant au même traitement, én régénérant à la même époque, des parties de forêt qui pourront avoir des exigences bien opposées.

Quel est l'objet principal de l'aménagement?.G'est de fixer l'époque et l'ordre des coupes annuelles; or cet ordre et cette époque ne dépendent pas. uniquement de la position respective des divers peuplements dont-une forêt se compose; ils dépendent en outre, et quelquefois principalement, de l'âge des bois, de la longévité probable des essences, et, par conséçuent, je ne saurais admettre qu'on ne tienne compte que de la première de ces circonstancis.

Il y a là, au moins en théorie, une erreur; je dis en théorie, parcè que dans la pratique on se préoccupe, tout en faisant le parcellaire, de la marche des coupes, et qu'il y a des cas où il est possible de prévoir que les exigences des règles d'assiette feront exploiter à la même époque, à cause de leur situation respective, des peuplements très-dissemblables d'ailleurs par l'âge ou d'autres caractères.

Ce sont précisément ces exigences des règles 
d'assiette et-la certilude où l'on est souvent, et en montagne particulièrement, qu'elles prévaudront, lors du règlement des coupes, sur les autres molifs, qui ont conduit certains agents à regarder comme devant être soumise à la même loi, et devant former l'élément fondamental du parcellaire, une portion de forêts circonscrite par des limites naturelles; mais il est clair d'abord que de tels principes, en admettant qu'ils fussent toujours justifiés dans les pays de montagnes, ne sauraient l'être dans les plaines, et il est non moins clair ensuite que, si dans la pratique il est permis à des agents trèsexercés de tenir compte, dans leur manière de procéder au parcellaire, des exigences des règles d'assiette, il n'en est pas de même en théorie, où l'on doit classer les idées et les opérations par rang d'importance, les développer et les exposer successivement et sans confusion, dans un ordre tel qu'on puisse bien en suivre la filiation.

ARTICLE VI.

DU NOUBRE ET DE LA FORME DES PIÉCES RELATIVES

AU PARCELLAIRE

et a la DESCRIPTION SPÉCiALE.

Dans mon opinion, il serait fàcheux que l'on imposât pour ces documents des modèles uniformes, sans avoir égard aux circonstances locales. Dans telle 
forêt, susceptible d'être divisée en un petit nombre de parcelles bien homogènes, le rapport sur un seul plan de toutes ces parcelles sera suffisant; dans telle autre forêt, au contraire, où il aura été nécessaire de former un nombre considérable de parcelles, à cause de l'irrégularité du peuplement ou des conditions de la végétation, il conviendra qu'indépendamment d'un plan d'ensemble on fournisse des plans de détail.

Pour la forêt de Cerisy (Manche) et poùr celle de Perseigne (Sarthe), on avait reconnu la nécessité de ces plans de détail : et comme l'irrégularité du peuplement était très-grande, même dans chaque parcelle envisagée isolément, et que le cahier descriptif n'aurait pas suffi pour montrer cette irrégularité autant qu'il était désirable, on prit le soin de figurer sur les plans partiels eux-mêmes, au moyen de pointillés et de quelques ánnotations sommaires, les principales nuances du peuplement.

Il serait, je le pense, très-utile que cet exemple fût imité.

Voilà pour ce qui concerne la représentation graphique du parcellaire.

Quant au cahier descriptif, il convient de n'y pas ménager les détails, tout en laissant en relief l'état moyen qui sert en définitive à fixer la destination d'une parcelle. On ne saurait d'ailleurs prescrire pour cette description une formule plutôt qu'une autre, la manière de décrire une parcelle devant être inspirée par l'état des lieux. 
Je donne, à titre d'exemple, un plan partiel et une description de parcelle que j'emprunte à l'aménagement de la forêt domaniale de Cerisy.

\section{PLAN}

ET DESCRIPTION D'UNE PARCELLE

Canton du Grand-Wez, parcelle A.

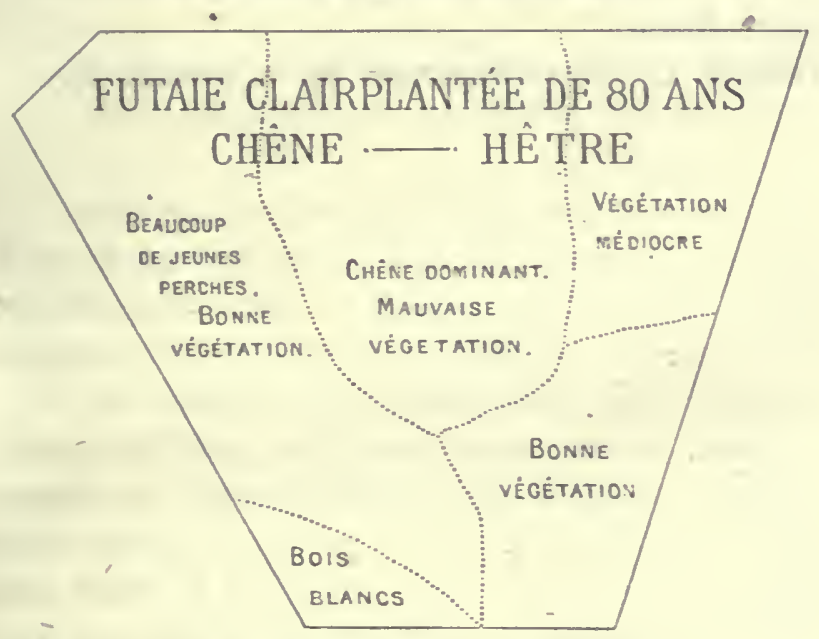

Contenance. 9 hectares 94 ares 70 centiares. Siluation. Plateau légèrement inclinó, à 300 mètres à peu près au-dessus du niveau de la mer. Exposition. Nord-ouest. Les vents sont à craindre. Sol. A la surface : Genêts, ronces, myrtilles, bruyères.

- A l'intérieur: Grès et schiste argileux, recouverts d'une couche végétale peu profonde, dont 
l'argile formé l'élément très-dominant, peu d'humus.

Essences. Chêne, hêtre, quelques bois blancs.

Age. 80 ans en moyenne.

Élat de la végétation et de la consistance. Futaie clair plantée peu régulière, d'une végétation mauvaise dans les parties où le chêne est dominant; on ne pense pas que ce peuplement puisse rester plus d'une vingtaine d'années sur pied sans dépérir. Vides. Néant.

Vidange. Les bois s'exportent par le chemin de... 


\title{
DEUXIÈME ÉTUDE.
}

\author{
DE L'EXPLÖITABILITÉ.
}

BUT ET DIVISION DE CETTE ÉTUDE.

On définit l'exploitabilité d'un arbre, d'un bois, d'une forêt, l'état dans lequel se trouve cet arbre, ce bois, cette forêt, au moment où l'on peut retirer de son exploitation les plus grands avantages.

Il est évident que la détermination de l'exploitabilité et de l'âge qui y correspond, est de nature à exercer une influence prépondérante sur les résultats de la culture forestière, et qu'elle serait, à ce titre seul, digne de toute l'attention de mes lecteurs. Cette attention, elle la mérite encore à cause des recherches nombreuses et difficiles qu'elle exige.

Dans l'agriculture proprement dite, l'exploitabilité des fruits est indiquée d'une manière précise par leur maturité. Aucun doute ne peut s'élever, en conséquence, sur l'époque à laquelle il convient au propriétaire d'effectuer la récolte, et un champ de blé, par exemple, est exploitable dans un temps prévu, fixé 
par la nature elle-même, et qui ne saurait varier, toutes conditions climatériques égales d'ailleurs, suivant la volonté de l'homme. Lorsque le soleil a doré les épis, on sait qu'il faut les faucher, sous peine de perdre les grains qu'ils contiennent. Le cultivateur est impuissant à hâter ou à retarder le moment de la moisson.

Pour un bois, les choses se passent bien autrement : outre que la maturité d'un bois n'est pas caractérisée par des signes extérieurs, sur lesquels aucune contestation ne puisse s'élever, je ferai remarquer qu'il n'est pas nécessaire d'attendre cette mạturité pour que l'exploitation de ce bois soit avantageuse; et que, suivant les convenances du propriétaire et la nature de la production qu'il recherche, l'exploitabilité peut être ou très-avancée ou très-retardće.

Les bois jouissent donc, sous le rapport que j'envisage, d'une sorte de privilége. On verra que dans'certaines circonstances ce privilége se manifeste par des bénéfices considérables; mais il est facile de com- prendre dès à présent qu'il doit avoir, dans tous les cas, pour effet de compliquer singulièrement les difficultés inhérentes à la fixation de l'exploitabilité forestière.

Si l'on examine la manière dont les particuliers, les communes et l'État traitent les forêts qu'ils possèdent, on reconnaîtra ce qui suit:

Les particuliers exploitent généralement en taillis, aussitôt que possible, presque toujours avant l'âge de 20 ans, les bois qui se prêtent à ce mode d'exploitation. Quant aux résineux qui s'y refusent complé- 
tement, ils les coupent à un âge qui ne dépasse guère 60 ans.

Les communes exploitent aussi en taillis, sauf de rares exceptions, les bois feuillus qui leur appartiennent; mais elles leur appliquent une plus longue révolution qui est le plus souvent de $\mathbf{2 5}$ ans, et elles y conservent de nombreuses réserves; leurs futaies résineuses sont exploitées de 80 à 90 ans.

L'État enfin exploite ses taillis, sous futaie et à 30 ans en moyenne; ses forêts, en futaie-pleine, restent sur pied jusqu'à 120 ans au moins.

Tels sont en général et à grands traits, les principes d'après lesquels est régi le sol forestier de notre pays.

Les intérêts des trois catégories de propriétaires qui possèdent ce sol, ne sont donc pas identiques, et si l'un s'accorde avec l'intérêt public, les autres doivent nécessairement s'en écarter.

Nontrer en quoi et pourquoi ces intérêts diffèrent; indiquer les moyens de les concilier, si cela se peut; ce sont là des points importants que nous aurons à étudier, quand nous aurons traité de l'exploitabilité à un point de vue théorique.

Comme toutes les opérations qui entrent dans l'économie forestière, la détermination de l'exploitabilité est subordonnée à deux ordres de circonstances bien distincts et qui tiennent: l'un, à la végétation, à ses lois naturelles; l'autre à des faits économiques, c'est-à-dire agricoles, industriels et commerciaux, aux convenances de la Société ou de l'individu, à l'es- 
pèce des produits qu'on veut obtenir, à la destination qu'il est possible de leur donner.

Je sais que dans la pratique, parmi les considérations qui servent à déterminer l'exploitabilité, celles qui se fondent sur les exigences de la végétation passent avant toutes les autres. S'il en était autrement, on s'exposerait à des travaux inutiles, en ce sens qu'ils pourraient reposer sur des hypothèses contradictoires avec les exigences mêmes de l'existence des hois.

Mais la logique de la pratique n'est pas toujours celle de la théorie.

La culture forestière n'emprunte sa raison d'être qu'au profit qu'elle est susceptible de fournir. L'appréciation de ce profit et de toutes les circonstances qui peuvent l'influencer, est donc celle qui se présente naturellement la première à l'esprit, quand on entreprend de classer les principes applicables à cette culture.

Cela posé, voici quelle sera la table des matières de cette étude $: 1^{\circ} \mathrm{De}$ l'exploitabilité, abstraction faite des exigences de la végétation et de la culture; $2^{\circ} \mathrm{De}$ l'exploitabilité dans ses rapports avec ces exigences; $3^{\circ}$ De l'exploitabilité dans ses rapports avec l'intérêtt des diverses catégories de propriétaires qui possèdent des bois en France; $4^{\circ}$ De l'exploitabilité dans ses rapports avec l'intérêt public 


\title{
CHAPITRE PREMIER.
}

\author{
exduewer \\ de l'exploitabilité, abstraction Paite des exigences \\ DE LA VEGÉTATION ET DE LA CULTURE.
}

La quantité des produits matériels, leur utilité, leur valeur vénale, et le rapport de cette valeur au capital dont elle émane, sont les divers objets que l'on a en vue, ensemble ou séparément, quand on cherche à retirer des bois les plus grands avantages.

A ces divers objets correspondent quatre sortes d'exploitations distinctes et relatives :

La première, aux produits en matière les plus considérables;

La deuxième, aux produits les plus utiles;

La troisième, au plus grand produit en argent;

La quatrième, au revenu le plus élevé par rapport au capital.

ARTICLE PREMIER.

DE L'ExploitabiLIté ReLATIVE AUX PRODUITS MATÉRIELS

LES PLUS CONSIDÉRABLes, OU DE L'EXPLOITABILITÉ ABSOLUE.

Quelles sont les lois générales sur lesquelles repose la recherche de l'exploitation absolue? 
Par quels moyens détermine-t-on l'àge qui correspond à cette exploitabilité ?

Quelle est son utilité pratique?

Examinons successivement ces trois questions:

$$
\$ 1^{\mathrm{er}} \text {. }
$$

Lois générales sur lesquelles repose la recherche de l'exploitabilité absolue.

Quel est l'âge auquel il convient d'abattre des arbres, soit isolés, soit en massif, pour qu'en admettant une régénération immédiate et une succession non interrompue d'arbres de la même espèce et soumis à des conditions semblables, on obtienne, dans un temps donné, les produits matériels les plus considérables?

Évidemmeńt, c'est comme si l'on demandait quel est l'âge auquel il convient d'exploiter des arbres, pour que le produit de cette exploitation, joint à tous ceux qu'auraient pu procurer, précédemment, lcs coupes d'amélioration, et divisé par le nombre des années comprises dans cet âge, présente le quotient le plus élevé.

L'exploitabilité absolue est donc indiquée par l’age où se réalise le plus grand accroissement moyen, et par conséquent:

Elle se présente dans la phase descendante des accroissements annuels. 
Elle correspond à l'age où l'accroissement annuel devient égal à l'accroissement moyen.

Ces trois propositions demandent quelques explications :

Si nous coupons un arbre par le pied, et si nous examinons les couches concentriques de la section transversale, nous reconnaîtrons que leur épaisseur, très-mince dans le principe, va en augmentant jusqu'à une certaine distance du centre; puis reste à peu près constante pendant quelques années; et diminue enfin de plus en plus, au point de devenir à peine perceptible, à mesure que l'on se rapproche de la circonférence.

Les conséquences qu'il y a lieu de tirer de ces caractères, pour la détermination de l'exploitabilité correspondante à la production matérielle la plus considérable, sont évidentes pour un forestier; il n'en est pas de même.pour les personnes étrangères à la sylviculture, et je crois que si on les interrogeait à ce sujet, elles ne répondraient pas, du premier coup, d'une manière satisfaisante.

Ces personnes seraient probablement disposées à établir d'abord une relation directe entre les phases de l'accroissement en volume de notre arbre d'expérience, et les variations de l'épaisseur des couches concentriques; elles supposeraient volontier's, qu'it partir du moment où cette épaisseur est devenue constante, l'accroissement annuel est resté stationnaire. - Ce serait là une grave erreur : Varennes de Fenille a eu soin de la prévoir, en faisant remar- 
quer qu'il faut se garder de confondre le grossissement avec l'accroissement annuel; attendu que celuici peut ne pas cesser de grandir, tandis que l'autre diminue.

Mais, ce que les personnes en question auraient de la peine à comprendre : c'est qu'il y ait perte dans le rendement, quand on retarde l'exploitation jusqu'au terme"de la végétation; c'est que, quelque pctite qu'elle doive être, il y ait avantage à sacrifier l'augmentation de volume que la végétation des dernières années serait encore susceptible de procurer au propriétaire. Habituées à.n'envisager les arbres que dans leur individualité, au lieu de les envisager dans une succession non interrompue et indéterminée de générations, il leur faudrait un certain ellort de réflexion pour apprécier les conséquences de la solidarité qui existe entre ces générations, solidarité dont l'absence rendrait inintelligible la théorie du plus grand accroissement moyen.

Enfin, cette solidarité consentie, et avec elle l'opportunité de renoncer aux dernières années de la végétation d'un arbre, parce qu'on est assuré de trouver plus qu'une compensation dans la production de celui qui le remplacera, il reste encore à démontrer que l'exploitabilité absolue tombe dans la phase descendante des accroissements annuels. Cette démonstration est facile : pour que l'accroissement moyen ne cesse d'augmenter, il suffit en effet que l'accroissement annuel soit plus grand que la moyenne des accroissements annuels antérieurs; or cette moyenne. 
est nécessairement toujours plus petite que le plus grand de ces accroissements, puisqu'elle est fonction non-seulement de celui-ci, mais, au mème tilre, des plus faibles, et entre autres, de ceux des premières années de la végétation.

Les deux premiers aphorismes que j'ai posés ne sont donc pas susceptibles d'ètre controversés; le troisiène en découle logiquement, en forme le corollaire, et s'y arrèter serait faire trop peu de cás du temps de ses lecţeurs.

Quelque simples que soient ces aphorismes, ils ne s'acceptent pas cependant, on vient de le voir, sans explications, et Varennes de Fenille, qui les a formulés le premier, a fait faire un progrès réel à la science de la sylviculture.

Toutefois, pour que ce progrès ne soit pas dépourvu d'utilité pratique, il est nécessaire d'admettre qu'en conformité des lois qui régissent le monde organique, les arbres n'arrivent au terme de leur existence qu'après avoir traversé une phase de décadence; que leur force vitale et leur accroissement annuel, qui en est l'expression, me vont pas en augmentant jusqu'au moment où ils sont surpris par la mort; et que si leur végétation ne présente pas toujours les trois phases, ascendante, stationnaire, descendante, qu'on s'accorde généralement à lui reconnaître, elle présente au moins les deux extrêmes. Cette hypothèse est indispensable, et je ne sache pas, au surplus, qu'il ait été fait contre elle aucune protestation plausible.

Les principes qu'on vient de développer s'ap- 
pliquent aussi exactement aux arbres isolés qu'aux arbres en massifs aux bois aménagés qu'à ceux qui ne le sont pas.

On a prouvé par exemple que, pour les arbres isolés, le plus grand accroissement moyen se produit dans la phase descendante de l'accroissement annuel. On le prouverait de la même manière pour les massifs ; seulement, lorsque dans ces derniers, les éclaircies enlèvent périodiquement une portion des arbres sur pied, il convient de supposer qu'elles ont lieu par annuités; et l'on doit, surtout, tenir compte de leurs produits, dans la comparaison des accroissements de deux années consécutives; car si on les négligeait, on arriverait infailliblement à un résultat tout à fait contraire à celui qu'indique le raisonnement.

Quant' à l'application aux bois aménagés des lois dont on n'a, jusqu'à présent, démontré la justesse que pour les bois qui ne le sont pas, elle est également incontestable : de sorte qu'étant données deux forêts de même étendue et dans des conditions semblables de végétation, partagées l'une en 60 coupes et l'autre en 120 , la production de cette dernière, quoique l'étendue de la coupe annuelle soit moindre de moitié, sera plus considérable que celle de la première, si le plus grand accroissement moyen ne se réalise qu'à l'âge de 120 ans. En termes plus précis, si ces deux forêts ont chacune 120 hectares, l'hectare qu'on coupera chaque année dans la deuxième, fournira plus de produits que les deux. hectares qu'on coupera chaque année dans la première. 
Voici un raisonnement algébrique très-élémentaire qui, à cet égard, ne laissera pas place au moindre doute.

On veut prouver que l'aménagement qui correspond au maximum de l'accroissement moyen est celui qui procure le maximum de produits matériels.

Soit $n$ l'àge de la révolution,

$v$ le volume d'un hectare parvenu à cet àge.

Le plus grand accraissement moyen sera $\frac{v}{n}$

Soit $c$ la contenance de la forêt ; la contenance de la coupe sera $\frac{c}{n}$ et le volume $\frac{c}{n} \times v$ ou $c \times \frac{v}{\mathrm{n}}$

Mais $\frac{v}{n}$ est le plus grand accroissement inoyen; donc toute révolution $n^{\prime} n^{\prime \prime} \ldots$ plus grande ou plus petite donnerait un accroissement $\frac{v^{\prime}}{n^{\prime}} \frac{v^{\prime \prime}}{n^{\prime \prime}} \ldots$ moindre que $\frac{v}{\mathrm{n}}$ et en conséquence, une quantité $c \frac{v^{\prime}}{n^{\prime}} c \frac{v^{\prime \prime}}{n^{\prime \prime}} \ldots$ plus faible que $c \frac{v}{n}$

\$2.

Des moyens par lesquels on détermine l'àge qui correspond à l'exploitabilité absolue.

Voici des arbres isolés de la même espèce, placés dans des conditions identiques de végétation, mais ayant des âges différents. Prenons le plus âgé, coupons-le par le pied. A l'inspection des couches 
concentriques, il nous sera facile de déterminer la grosseur qu'il avait aux diverses époques pour lesquelles nous voulons établir son volume. Ayant la grosseur, il nous faudra encore la longueur à ces mêmes époques. Si l'arbre est verticillé, cette longueur sera facile à trouver, puisque la distance entre deux verticilles successifs représente la pousse d'une année, et que pour avoir, par exemple, la longueur de l'arbre il y a $\mathbf{1 0}$ ans, il suffira de retrancher de la longueur totale actuelle, celle des $\mathbf{1 0}$ derniers verticilles. S'il s'agit de bois feuillus, ce n'est que par des tâtonnements que nous pourrons arriver au but de nos recherches : ainsi, l'accroissement d'un arbre se constituant avec des couches annuelles. superposées, il est aisé de comprendre qu'en rognant successivement la tige, on augmentera successivement aussi le nombre des couches concentriques de la section; et qu'à l'apparition de chaque couche nouvelle correspondra la hauteur à un âge qu'on déterminera sans peine, puisqu'on connaîtra l'âge actuel.

Nous pourrons donc nous procurer les deux éléments, longueur et épaisseur, relatifs à un âge quelconque, et; avec ces deux éléments, calculer le volume de la tige de notre arbre à cet âge, en le considérant soit comme cône, soit comme cylindre. Des facteurs de conversion que nous établirons par les procédés qu'enseigne la stéréométrie, nous fourniront le volume réel, et il ne nous restera plus qu'à diviser le volume trouvé à différents âges par ces âges mêmes, pour distinguer en comparant les 
quotients, l'àge du plus grand accroissement moyen .

Pour plus de sûreté, l'opération que je viens d'appliquer à un arbre, on l'applique à plusieurs, et on prend ensuite la moyenne des accroissements moyens maxima.

Voici maintenant des arbres en massif : c'est un taillis dans lequel il n'y a pas'lieu de faire des coupes intermédiaires, c'est-ì-dire des nettoiements ou dès éclaircies. On pourrait déterminer l'àge de l'exploitabilité absolue par le même procédé. Mais comme pour diminuer les chances inévitables d'erreur que l'on court toujours, lorsqu'on applique à un trèsgrand nombre d'arbres les lois d'accroissement constatées sur une partie seulement d'entre eux, il faut que cette partie soit par rapport au tout la plus grande possible; et comme, dans un massif, cette considération entraînerait l'abatage d'un trèsgrand nombre de sujets, on emploie le procédé suivant qui est moins compliqué, plus expéditif, et souvent plus sûr.

Au lieu d'expérimenter sur des arbres isolés, on expérimente sur des portions du massif. On choisit donc, dans des conditions régulières de végétation, des places d'essai, différentes par l'âge, mais semblables quant aux autres éléments de la production. On cube les volumes qu'elles contiennent, et l'on divise ces volumes par les àges correspondảnts.

Cette méthode est celle qu'on ne peut se dispenser d'employer dans les massifs, soit en taillis, soit en futaie, qui sont assujeltis à des nettoiements et 
éclaircies périodiques; seulement, on a soin d'ajouter au volume de chaque place d'essai, celui des coupes intermédiaires effectuées précédemment; de sorte que si des éclaircies avaient eu lieu tous les 10 ans, à partir de 20 ans, il faudrait ajouter au volume des bois de 30 ans, le produit d'une éclaircie (à 20 ans), au volume des bois de 40 ans, les produits de deux éclaircies (à 20 et à 30 ans), etc. Les quotients de ces sommes par les âges actucls exprimeraient les accroissements moyens, et au plus grand de ces quotients correspondrait l'âge le plus rapproché de celui de l'exploitabilité absolue.

Les opérations que je viens de décrire très-rapidement sont simples en théorie. Dans la pratique, elles rencontrent de grandes difficultés causées principalement par l'embarras de trouver des portions de massifs qui soient dans un état de régularité convenable, et dans des conditions de végétation semblables à celles des peuplements, auxquels devraient être appliqués les résultats des expériences dont elles auraient été l'objet.

L'exploilabilité relative aux plus grands produits matériels varie pour une même essence, suivant les sols, les climats, la situation, etc., toutes choses trèsvariables souvent de leur côté, dans la même forêt. Il n'est pas aisé de discerner l'état moyen au milieu de ces variations, et c'est cependant de la découverte de cet état-moyen que dépend le succès des essais. Toutefois, il n'y a pas de difficulté insurmontable pour la sagacité d'un forestier qui a du coup d'œil 
ot qui est expérimenté, lorsque les peuplements présentent les élëments indispensables sous le triple rapport de la régularité, de la consistance des massifs et de la gradation des âges. C'est là mallieureusement ce qui manque en France à presque toutes nos futaies. Aussi est-il bien peu de futaies dans lesquelles l'àge d'exploitabilité puisse être fixé d'après des bases certaines. Pour nos taillis, la recherche des lois d'accroissement est plus facile. Pourtant elle n'a été faite que très-exceptionnellement. Nous verrons au surplus qu'elle est bien loin d'aroir une aussi grande importance que dans les futaies.

Des tables de production résultant d'expériences effectives et qui indiqueraient, pour des conditions déterminées de végétation, la marche que suit l'accroissement des principales "essences de notre sol forestier, constitueraient un précieux document, et il serait très-désirable que l'on s'en occupât. L'administration seule pourrait entreprendre un travail de cette nature. Ce ne serait, il est vrai, pas trop d'un siècle pour le terminer; mais les résultats immédiats auxquels l'état actuel de nos forêts permettrait d'arriver, auraient déjà un grand intérêt et une grande utilité pour la détermination de l'exploitabilité dont nous nous occupons en ce moment. Ces études que je conseille, l'administration forestière badoise les a commencées depuis longtemps dans les forêts dont la gestion lui est confiée. Elles ne sont donc pas impossibles.

Mes lecteurs s'effrayeront peut-être de la mul- 
titude de comptages et de calculs que semble comporter la recherche de l'exploitabilité absolue, et se défieront de la possibilité d'arriver dans ces évaluations à un degré suffisant d'exactitude. De telles appréhensions ne seraient pas fondées: dans la pratique, la phase de la végétation sur laquelle portent les expériences est singulièrement réduite, soit par le choix du mode d'exploitation, soit par les exigences de la culture; et pour ce qui est de la précision des cubages, ceux-là seuls pourraient en douter qui ignorent les procédés de la dendrométrie. L'emploi de ces procédés peut être expéditif et certain, comme le prouvent les exploitations faites dans diverses forêts, conformément à la possibilité résultant des cubages de, l'aménagement. Ainsi, dans la forêt de Haguenau, le produit des coupes de la première décennie a cadré parfaitement avec la possibilité déterminée par l'aménagement. Il en a été de même dans la forêt de Cerisy (Manche), et il en sera de même toutes les fois que les comptages et cubages seront faits par des agents attentifs.

Au reste, on aurait grand tort de contredire l'utilité d'un principe, en alléguant les difficultés de son application. En sylviculture, comme dans toutes les sciences et dans tous les artś spéciaux, la pratique parvient rarement à réaliser, à appliquer exactement, complétement, les enseignements de la théorie. Ce n'est pas une raison pour qu'elle les repousse, et celui qui s'inscrirait en faux contre les principes de l'aménagement des forêts, par cette seule raison 
qu'ils ne trouvent pas toujours leur application, ne serait pas plus sage que-le mécanicien qui nierait l'utilité de la mécanique rationnelle, parce que les données qu'on y puise sur la résistance des matériaux, ne sont pas toujours d'accord avec le résultat des épreuves que l'on fait subir à ces derniers.

\section{$\$ 3$.}

Utilitê pratique de l'exploitabilitẻ absolut.

J'ai fait remarquer, au début de mon travail, que le profit qu'on pouvait retirer de l'exploitation forestière, servait de fondement aux règles qui régissent cette exploitation. Mais il n'y a profit que là oủ il y a utilité produite, et comme je n'ai pas tenu comple jusqu'ici de cette utilité, on pourrait douter de l'opportunité des longues considérations dans lesquelles je suis entré, au sujet d'une exploitabilité dont les avantages, pour la satisfaction de nos besoins, n'ont pas encore été développés.

La quantité plus ou moins grande des produits matériels qu'un bois est susceptible de fournir annuellement, serait dépourvue de tout intérêt, si elle était indépendante de l'utilité et de la valeur de ces produits; elle ne mérite d'être prise en considération que parce qu'elle a des rapports plus ou moins directs avec cette utilité et cette valeur; on s'étonnera donc peut-être que je n'aie pas pris ces rapports pour point de départ de mes études. 
Je ne l'ai pas fait, parce que j'ai présumé que la clarté de mes déductions en souffrirait.

Je reconnais, sans doute, que réduite à ellemême, envisagée isolément, la théorie de l'exploitabilité absolue serait une pure abstraction comparable à un chiffre qu'on aurait séparé de l'objet auquel il empruntait sa signification ; mais nous verrons plus tard que l'utilité et la valeur des produits ligneux sont, dans beaucoup de cas, proportionnelles à leur quantité, et qu'en conséquence, la théorie de l'exploitabilité absolue est souvent admissible. Elle exige d'ailleurs qu'on précise la marche de la végétation, qu'on en fasse connaître les loị et les résultats matériels, et ce sont là des données dont on ne saurait se priver, quels que soient le but que l'on se propose et les moyens que l'on préfère pour y arriver. Il n'était point, enfin, sans importance d'avoir un terme de comparaison aussi fixe que possible pour les exploitabilités de différentes natures que j'avais à étudier, et il n'en existait pas, ce semble, de meilleur que l'exploitabilité absolue.

ARTICLE II.

DE L'EXPLOITABILITÉ RELATIVE AUx PROdULTS LES PLUS UTILES.

L'exploitabilité relative à la production la plus utile, est celle qu'il est le plus difficile de déterminer. Il ne suffit pas, comme on pourrait le croire au 
premier abord, de -transformer en marchandises, les produits des arbres d'expérience ou des places d'essai, pour juger ensuite, d'après le prix de ces marchandises, de leur utilité respective. Ce prix est; sans doute, un élément d'appréciation; mais on ne doit en user qu'avec réserve, sous peine de s'exposer: à de grossières erreurs.

L'utilité des choses est, en effet, loin d'être toujours proportionnelle à. leur valeur. Le bois de houx qui, tous les forestiers le savent bien, est une essence digne de fort peu d'intérêt, se paye sur place, dans telle forêt que je pourrais citer, jusqu'à 250 francs le mètre cube. Personne assurément ne s'avisera de prétendre qu'à un point de vue général, ce bois soit aussi utile que le chêne qu'on vend en moyenne 40 à 50 francs le mètre cube. Un même objet, qui vaut beaucoup aujourd'hui, vaudra peutêtre très-peu demain, sans que son utilité ait cependant diminué. Enfin, il y a des utilités entièrement gratuites : l'air, l'eau, les bois aussi quelquefois.

On peut n'altendre de ces derniers que de l'agrément; s'ils en procurent, ils seront utiles; on peut en attendre une protection contre les vents, les avalanches, etc., etc. : ces bouquets de bois qui, sur les rampes escarpées de nos Alpes, ont pour destination de barrer les torrents et de les éloigner des habitations qu'ils menacent d'emporter, rendent, sans contredit, de grands services, produisent des utilités; ces utilités n'ont pas de valeur vénale, et cependant, elles sont incontestables. 
Il est donc nécessaire de chercher une autre base que le prix, pour l'appréciation du maximum d'utilité des produits forestiers; mais où la trouver? Entre l'utilité du taillis qui sert de remise au gibier, et celle des forêls séculaires de nos hautes montagnes, dont la fonction est de garantir les contrées inférieures contre la fureur des éléments, combien n'y a-t-il pas d'autres services immatériels et souvent gratuits rendus 'par les bois, services s'appliquant au caprice, à l'agrément, à des besoins plus ou moins impérieux? Entre l'échalas qui sert de tuteur à la vigne et le mât de hune qui résiste à l'effort de la tempête, el sous lequel s'abritent les intérêts de notre commerce et quelquefois de notre gloire, combien ne pourrait-on pas compter de destinations différentes ayant chacune des avantages spéciaux? Où trouver un criterium, une mesure qui permette de classer tous ces services par rang d'importance?

Pour le vigneron, l'échalas est le plus utile de tous les produits. Pour celui qui manque de combustible, le fagot est bien plus utile que le plus beau bois' des îles, si celui-ci n'est bon qu'à la fabrication des meubles. Pour le propriétaire qui veut faire argent de ses produits, le bois le plus cher est le plus avantageux.

On voit toutes les raisons qui s'opposent à ce qu'on fixe des règles pour juger de l'exploitabilité relative aux produits les plus utiles. Cependant, on peut admettre en principe, qu'à un point de vue général et sauf de rares exceptions, les arbres les plus utiles sont ceux qui ont les dimensions les plus grandes 
et les propriétés mécaniques ou physiques les p̉lus développées.

Les dimensions ont sur l'utilité d'un morceau de bois une influence bien évidente, puisque c'est d'elles que dépend le nombre des usages auxquels il est possible de l'affecter : et il est clair, par exemple, qu'à volume égal, plusieurs rondins de chêne propres au chauffage seulement, seront moins utiles qu'une seule pièce de bois de la même essence, dont on tirera du bois de chauffage, du bois de travail, du bois de charpente.

Le développement des propriétés mécaniques et physiques n'a pas une moins grande influence; or, l'expérience tend à prouver que la puissance calorifique, la densité, la cohésion et l'élasticité, suivent une marche ascendante jusqu'à un âge avancé, qui ne s'écarte pas beaucoup de celui qui correspond au plus grand accroissement moyen : d'où il résulte qu'à dimensions égales, du bois de 20 ans est moins précieux que du bois de 100 ans.

Henri Cotta raconte, quelque part, que dans la Suisse saxonne, des habitations ont duré 20 ans à peine, parce qu'on y avait employé de jeunes 'bois, tandis que des édifices construits il y a plus de 100 ans, avec des bois âgés, sont encore dans un parfait état de conservation. Ce fait est frappant; il montre à quelle perte énorme d'effet utile on s'expose par des exploitations prématurées ${ }^{1}$.

Je faisais observer, dans l'article précédent, qu'il

1. Le laricio est le moins durable de tous les bois, quand il est 
serait très-désirable que l'on s'occupât activement de la formation de tables de production indiquant, pour des conditions déterminées de végétation, la marche de l'accroissement des principales essences de notre pays. Il ne le serait pas moins que l'on établît, par des expériences consciencieuses, l'influence que l'âge, le sol, le climat et le mode d'exploitation, exercent sur les qualités de cés essences. Le chêne de la Meurthe est moins cstimé pour le chauffage que celui de la Bourgogne, en sait-on la raison? Le chêne du Nord dure, dit-on, beaucoup moins que celui du Midi; on assure que les vaisseaux construits avec le premier, sont hors de service au bout de 7 ou 8 ans, en moyenne, tandis que ceux qui sont construits avec du bois de Provence, se conservent plus de 15 ans. Certes, ce sont là des assertions qui réclameraient une sérieuse vérification. Quels services l'administration ne rendrait-elle pas au commerce et à l'industrie, si elle pouvait dire : Dans cette région, le bois est propre à tel usage; il se distingue dans celle-ci par telle qualité? etc...

Il existe bien quelques principes relativement aux influences que je voudrais que l'on étudiât, mais ils ne reposent que sur des observations fort incomplètes et rarement comparables entre elles.

Les bois de grandes dimensions ne sont pas seulement recommandables par la variété et l'importance

employé comme poteau télégraphique, parce qu'alors il est trop jeune, tandis que lorsqu'on ne l'exploite qu'à l'àge de 200 ans, il est presque incorruptible. (Note de la, $2^{e}$ édition.) 
des usages aunquels ils peuvent servir; ils le sont également par les éléments de bien-être que procurent à la classe ouvrière les nombreuses manipulations auxquelles ils donnent lieu.

L'exploitabilité relative aux produits les plus utiles est, cn définitive, susceptible de varier suivant une. foule de circonstances que je ne me flatte même pas d'avoir énumérées, et de se présenter tantôt avant, tantôt après l'exploitabilité absolue; il faut 10 ans pour faire un cercle; il en faut $\mathbf{2 5 0}$ pour que le pin de Riga puisse faire un mât de vaisseau.

Néanmoins, cette exploitabilité correspond à un àge qui ne saurait, dans la plupart des cas, s'éloígner beaucoup de celui du plus grand accroissement moyen.

Lorsqu' on recherche les dimensions que doivent avoir les bois pour atteindre leur maximum d'utilité, il importe de ne pas perdre de vue que l'utilité serait un vain mot, si elle ne pouvait être mise à la portée du consommateur.

L'état des voies de vidange est donc appelé à influer singulièrement sur le résultat de cette recherche.

Que si l'on ne considérait l'exploitabilité relative aux produits les plus utiles, que dans ses rapports avec l'intérêt du propriétaire qui ne consomme pas son bois et qui le vend, on pourrait regarder le prix de ces produits comme l'expression exacte de leur utilité; et, par suite, l'exploitabilité dont nous venons de nous occuper, se confondrait avec celle du plus grand produit pécuniaire, dont je vais parler. 


\section{ARTIGLE III.}

DE L'EXPLOITABILITÉ RELATIVR AU PLUS GRAND PRODUIT EN ARGENT.

La marche à suivre pour déterniner l'âge auquel il faut abattre des bois, quand on veut en retirer le plus grand produit en argent n'est pas embarrassante, du moment que l'on est fixé sur les rendements, en matière et en marchandises, des arbres d'expérience ou des places d'essai. Les prix du commerce appliqués à ces marchandises, défalcation faite des frais d'exploitation, de façon et de transport ${ }^{1}$, établiront exactement leurs valeurs; les quotients de ces valeurs par les âges correspondants, donneront les revenus moyens, et le plus grand de ces quotients indiquera l'époque de l'exploitabilité cherchée.

La chose essentielle, dans cette opération, afin d'éviter les erreurs, est d'apprécier sainement le prix vénal d'une marchandise dans les éléments qui le constituent; or, cela n'est pas facile, car le prix du bois est exposé à de très-grandes fluctuations, et il s'agit, au cas particulier, d'adopter non le prix du jour, le prix accidentel; mais le prix moyen, le prix normal. Quelques exemples feront mieux comprendre ma·pensée :

1. Dans l'évaluation des frais de transport, on tiendra comple de l'influence que l'amélioration des voies de vidange pourrait avoir sur ces frais. 
J'ai cité, tout à l'heure, le prix du houx comme un exemple de la valeur excessive que pouvaient alteindre des objets d'une utilité plus que contestable d'ailleurs. Eh bien! si le bois dont on voudrait régler l'exploitation renfermait des sujets de cette essence, en conclurait-on qu'il convient de les multiplier? Une réponse affirmative serait une erreur grave; car ce prix élevé tient précisément à la rareté de l'essence en question, aux soins que, dans l'intérêt de la sylviculture, on apporte à l'extirper, et il est compréhensible que, pour peu qu'on en favorisât la multiplication, sa cherté disparaîtrait. On fabrique, dans certaines localités, des allumettes avec du bois de tremble, et on en tire, de cetle manière, un excellent parti. Serait-ce une raison pour convertir nos forêts en trembles? - Évidemment non, car il en serait du tremble comme du houx; son prix ne tarderait pas à être avili.

Ce qu'il faut donc examiner avec attention, apprécier avec sagesse et prendre pour base de sonévaluation, c'est l'importance des débouchés qui seule peut garantir le maintien du prix vénal; et on doit rejeter dans les considérations tout à fait secondaires, les circonstances accidentelles et exceptionnelles dont l'influence, sur la valeur des produits, ne'saurait être durable."

Si l'on suppose le prix du bois constant, c'est-àdire indépendant de l'âge, l'exploitabilité relative au produit pécuniaire le plus élevé, suivra les mêmes lois que l'exploitabilité absolue. Elle se réaliserait, 
au contraire, plus ou moins longtemps après, dans l'hypothèse où le prix du bois s'élèverait avec l'àge; et plus ou moins łontemps avant, dans le cas où ce prix subirait une diminution, malgré le mouvement ascendant de la végétation.

\section{ARTICLE IV.}

De L'EXPLOITABILITÉ RELATIVE AU REVENU LE PLUS ÉLEVÉ PAR RAPPORT AU CAPITAL.

$\S 1^{\text {er }}$.

Considérations générales sur la valeur et le profit des capitaux, et spécialement des fonds de bois.

Nous n'avons examiné, jusqu'à présent, l'exploitabilité forestière qu'au point de vue de la quantité, de l'utilité, et de la valeur absolue des produits. La quantité et l'utilité des choses intéressent presque exclusivement le consommateur; leur valeur touche surtout le producteur; mais ce qui lui importe principalement, c'est le rapport qui existe entre cette valeur et le capital dont elle émane; aussi, les spéculateurs qui ont à faire emploi d'un capital pécuniaire, cherchent-ils à l'affecter à l'entreprise qui leur promet le bénéfice le plus grand, c'est-à-dire un profit qui soit, par rapport au capital, le plus élevé possible. Cette préoccupation légitime se rencontre chez le propriétaire de bois, et l'on conçoit qu'on ne 
puisse y satisfaire qu'en recourant à une exploitabilité bien différente de celles que nous avons étudiées précédemment.

On a souvent défini l'exploitabilité dont il s'agit : l'exploitabilité relalive à la rente la plus élecée.

J'adopterai aussi cette définition, quoiqu'elle ne soit peut-être pas irréprochable.

Ce qui caractérise l'exploitabilité qui va faire l'objet de notre examen, c'est donc l'intervention de la valeur capitale parmi les éléments à prendre en considération, quand on veut déterminer l'àge auquel il convient d'abattre des bois. Nous ne nous sommes encore occupé que du produit, sans songer à l'agent de production et au prix qu'on y attache dans les, transactions commerciales; or, cet agent a, comme les autres capitaux, une valeur commerciale, et il ne saurait dès lors être négligé par le spéculateur qui se propose de fixer, pour l'exploitation d'un bois, l'époque la plus favorable à ses intérêts.

Je dis que les capitaux ont une valeur commerciale. Pour l'intelligence de ce que j'aurai à exposer relativement aux fonds de bois, il est nécessaire de rappeler comment se constitue et fonctionne cette valeur.

On désigne sous le nom de capilaux toutes les choses, tous les objets qui servent ou qui contribuent à la production; ce sont donc en général des instruments de travail; leur valeur repose sur les services productifs qu'ils sont susceptibles.de rendre; elle s'établit, dans le commerce, en multipliant le prix 
net de ces services par un facteur convenu. Ce facteur varie suivant des circonstances nombreuses dont les principales sont la sécurité du placement et l'abondance des capitaux disponibles; mais il ne monte guère au-dessus de 40, tandis qu'il peut descendre beaucoup plus bas.

Les fonds de terre participent de la nature des capitaux, et dans le langage ordinaire, on s'accorde. à les ranger dans la même catégorie.

Qu'est-ce que le profit net? - C'est l'excédant du produit pécuniaire sur les frais de production ${ }^{1}$, d'où il résulte que les capitaux dont les services productifs seraient juste suffisants pour couvrir les frais de production, n'auraient aucune valeur commerciale, ce qui, soit dit en passant, ne les empêcherait pas d'être souvent fort utiles. Ainsi, qn'on suppose un fonds de terre dont le produit suffirait à peine à l'entretien du cultivateur et de ses instruments de travail, il est clair que, commercialement, ce fonds sera sans valeur; mais dès qu'il y a un profit net quelconque, la valeur capitale surgit, et pour l'apprécier, pour la déterminer, il n'y a qu'à multiplier ce profit par le facteur exprimant la relation convenue dont je viens de parler, la relation consentie entre le profit net et le prix de l'agent qui sert à le produire. Pour une terre arable, le profit net sera multiplié par 40; pour un bois, par 33; pour un capital

1. Il est bien entendu que dans ces frais est compris le bénéfice de l'entrepreneur, quand il y a un entrepreneur. 
employé à l'armement d'un navire, par 15, elc.

Ce profit net qui sert à l'évaluation des capitaux, indique en même temps ce qu'ils coûtent à l'industriel qui ne demande que la faculté d'en user, et il porte alors le nom générique d'intérêt (comprenant le loyer, le fermage, etc.). On suppose en effet que ce profit net est la conséquence nécessaire de l'intervention du capital, qu'il lui est, en quelque sorte, inhérent, et qu'il est dù, dès lors, au propriétaire, soit que celui-ci emploie lui-même son capital, soit qu'il le fasse fructifier par d'autres mains. II se pourrait certainement que l'emprunteur ne trouvât point dans les services du capital qu'on lui aurait prêté, cet excédant qui constitue le revenu du propriétaire. Cela ne prouverait rien contre ce que j'ai avancé à propos des rapports qui existent entre les profits nets et les capitaux. Cela prouverait seulement que le capital dont il s'agit aurait été surévalué ou mal exploité.

D'après ces considérations, la valeur d'un capital ne tiendrait pas à celle de ses parties intégrantes; elle serait intangible, extrinsèque, elle se lierait au revenu; elle diminuerait et progresserait avec lui.

Il est cependant connu de tout le monde que la valeur commerciale d'une forêt, n'est pas toujours arec le revenu net qu'on en retire, dans le rapport adopté pour les placements de l'espèce, et qu'elle dépasse quelquefois le chiffre que l'on obtient par la capitalisation de son revenu au taux desdits placements. 
D'ou cela provient-il ? Comment concilier ce fait incontestable avec la proposition précédente?

Essayons de répondre à ces questions :

Parce que le revenu net sert de fondement au prix que l'on attache à un capital, ce n'est pas nécessairement une raison pour que le revenu étant nul, les objets qui constituent le capital soient frappés de non-valeur; ils ne le seraient que dans l'hypothèse où ils ne pourraient être soustraits à la destination qu'on leur avait donnée, pour être affectés à un autre emploi. Voici des pierres, du fer, du bois avec lesquels on a construit une maison : si cette maison ne se louait à aucun prix, elle perdrait toute valeur comme capital-maison, capital immobilier; mais les matériaux dont elle se compose, séparés les uns des autres, rentreraient dans la catégorie des produits, reprendraient ainsi une partie de leur valeur primitive, et pourraient, sous une autre forme, fonctionner comme capital.

Veut-on encore un exemple? - Il nous est fourni par un bateau que l'on aurait transporté dans l'intérieur des terres. Sur le bord de la mer, ce bateau constituait un capital précieux; dans les conditions où on l'a placé, il a perdu comme capital-bateau toute valeur, et, cependant, il est probable qu'on en tirera quelque profit en le dépeçant.

Les choses qui fonctionnent comme capital, peuvent donc avoir et ont en effet, presque toujours, une valeur propre, indépendante de celle qu'elles empruntent à leur fonction; mais la première est 
ordinairement beaucoup plus petite que la seconde, et, par conséquent, elle ne saurait exercer aucune influence sur le prix qu'on attache à ces choses dans les transactions commerciales.

Les bois seuls peut-être font exception à cette règle.

Si le fonds de bois étail fixe, immutable, il est certain que son prix vénal se réglerait invariablement comme celui des autres capitaux et particulièrement des fonds de terre, d'après son, revenu net; mais le fonds de bois peut, dans une très-grande mesure, être transformé : de fixe, il peut devenir circulant; d'immeuble il peut devenir meuble, et ces propriétés qu'il possède à un degré exceptionnel, lui donnent une valeur intrinsèque, pour ainsi dire, indépendante du revenu qu'on en retire, et qui est susceptible de s'élever, au point-de dépasser le chiffre que l'on obtiendrait, en capitalisant ce revenu au taux des placements en bois. Comment s'établit cette valeur? - C'est bien simple : d'après l'usage que l'on ferait de la portion du capital que l'on aurait mobilisée. On sait, par esemple, que l'intérêt des capitaux qui se prêtent dans le commerce aux mêmes conditions de solidité que celles des placements en bois, est de 3 pour 100 . Eh bien! si le revenu net d'une forêt descendait au-dessous du profit qu' on retirerait de cette forêt, en transformant sa superficie en argent, il y aurait, à faire cette transformation, un avantage aisément appréciable. 
Le point que j'essaye d'éclairer est véritablement la clef de la théorie de l'exploitabilité relative à la rente la plus élevée. Qu'on me permette donc d'insister, et de montrer en quoi la formation du capital-forêt diffère de celle des autres capitaux et notamment des fonds de terre.

Le propriétaire d'une terre arable dépense ordináirement, soit pour ses besoins, soit pour ses plaisirs, le revenu net qu'il en retire. Supposons, cependant, que sa position de fortune et ses goûts lui donnent la possibilité et lui inspirent le désir de le faire valoir; supposons aussi qu'à raison de la solidité du placement, il soit disposé à se cọtenter de l'intérêt qui correspond au taux des placements en fonds de terre. Pour parvenir à ses fins, il peut faire l'une des deux opérations suivantes : ou bien augmenter par une nouvelle acquisition l'étendue de ses propriétés, ou bien améliorer celles qu'il possède déjà. Il choisira probablement ce dernier moyen, parce qu'il est plus commode que l'autre, si en employant son revenu à des améliorations, il prévoit qu'il augmentera son fermage d'une quantité égale à l'intérêt de ce revenu; et il continuera, chaque année, le même emploi, jusqu'au moment où l'augmentation du profit net cessera de compenser la perte d'intérêt du revenu.

A partir de ce moment, il cherchera pour ses épargnes une autre destination; il s'en servira, par exemple, pour augmenter l'étendue de ses biensfonds. 
Le propriétaire d'une forêt ne doit pas agir différemment; pour lui, l'accroissement d'une année, c'est le revenu; il ne consentira à l'immobiliser, à l'incorporer au capital, que dans le cas où l'accroissement de l'année suivante lui offrira un dédommagement.

Jusque-là, les deux capitaux, fonds de terre et fonds de bois, donnent lieu à des spéculations identiques; mais ce qui distingue le fonds de bois de la terre arable, c'est que, dans celle-ci, le revenu incorporé au capital ne se retrouverait plus, si une augmentation proportionnelle du revenu net ne se produisait pas ${ }^{1}$; tandis que, pour le fonds de bois, il se conserve et se retrouve, lors même que son immobilisation ne procure pas les avantages qu'on en attendait. Le propriétaire d'une forêt ne perd dans ce cas que l'intérêt de son-revenu; seulement, il arrive que le rapport du revenu au capital engagé dans cette forêt, descend au-dessous du taux des placements qui sont dans les conditions de solidité que ledit propriétaire recherche; et dès lorś il convient à ce dernier de distraire de son capital engagé toute la portion qu'il considère comme inerte, comme dormante, en ce sens qu'elle ne produit pas un revenu équivalant à l'intérêt qu'elle comporte.

1. J'ose affirmer qu'il n'est pas un champ en France qui vaille ce qu'il a coùté, qui puisse s'échanger contre autant de travail qu'il en a exigé pour être mis à l'état de productivité où il se troure.

(Bastiat, Harmonies économiques.) 
Ces considérations générales prouvent que la valeur effective, réalisable, d'un fonds boisé peut dépasser la somme qu'on obtiendrait, en capitalisant le revenu net de ce fonds boisé au taux des placements; elles prouvent que le rapport entre ce revenu et cette valeur peut être moins grand que celui admis entre les profits et les capitaux de l'espèce. Il existe, par conséquent, des fonds boisés dont il est permis de dire qu'ils ne rapportent que 2, que 1 pour 100 . En existe-t-il qui rapportent plus de 3 pour 100, en supposant que 3 pour 100 soit le taux ordinaire des placements en fonds boisés? Non, car si en s'appuyant sur le.bénéfice qu'il y aurait à soustraire une partie d'un fonds boisé à sa destination naturelle, on parvient, dans certaines circonstances, à faire accepter ce fonds boisé pour une valeur plus grande que celle qui résulterait de la capitalisation de son revenu net, on rirait évidemment au nez de l'acquéreur qui se fonderait sur l'inconvénient pécuniaire qu'entraînerait ce changement de destination, pour payer un fonds boisé au-dessous de sa valeur capitale.

En résumé, la valeur commerciale d'un fonds boisé peut se régler d'après un taux inférieur au taux de placement des capitaux de cette nature; elle ne peut pas se régler à un taux supérieur; et ce que je veux, en exposant la théorie de l'exploitabilité relative à la rente la plus élevée, c'est indiquer les moyens de faire fonctionner la propriété forestière, d'après le taux de placement qu'on accorde aux capitaux qui 
présentent les mêmes conditions de solidité et les mêmes avantages qu'elle.

Entrons maintenant en matière :

J'examinerai successivement l'exploitabilité relative à la rente la plus élevée, dans son application à un arbre isolé, à un massif non aménagé, et à un massif aménagé.

\section{$\$ 2$.}

De l'exploitabilité relative à la rente la plus élevée, dans son application à un arbre isolé.

Il y a deux suppositions à faire, savoir : $1^{\circ}$ celle où cet arbre occuperait un terrain vague, inculte, qui lui offrirait gratuitement l'espace que réclamerait son développement; $2^{\circ}$ celle où il serait, aı contraire, situé dans un champ sur lequel il ne pourrait pas s'étaler, sans diminuer proportionnellement l'étendue des cultures environnantes.

Première hypothèse. - L'arbre a atteint l'âge où il commence à avoir une valeur échangeable; - il a quinze ans; doit-on le couper ou le laisser sur pied? - Dès que l'on pose cette question, l'on admet, chose essentielle, - que les conditions d'aisance du propriétaire lui permettent d'épargner au moins une partie de son revenu annuel, et que, s'il exploitait son bois aujourd'hui, ce ne serait pas pour en dépenser le prix. Ce qu'il cherche donc, c'est le placement le plus avantageux, et il le cherchora jusqu'à ce que 
les exigences de la vie l'empêchent de continuer ou le forcent de diminuer ses épargnes.

Si son arbre reste sur pied pendant encore un an, par exemple, il acquerra une plus-value. Cette plusvalue sera-t-elle au moins équivalente au profit que lui procurerait une exploitation immédiate? - C'est là ce qu'il veut savoir; il y parviendra en comparant la valeur nette que son arbre serait susceptible d'acquérir dans un an à la somme représentant: $1^{\circ}$ la valeur nette actuelle; $2^{\circ}$ l'intérêt de cette valeur pendant un an; $3^{\circ}$ la valeur, à un an, du jeune plant qui remplacerait l'arbre existant.

Si le premier terme de la comparaison était plus petit que le second, il faudrait en conclure que tout délai dans l'exploitation serait préjudiciable au propriêtaire.

Si les deux termes étaient égaux, le propriétaire ne trouverait aucun bénéfice à maintenir son arbre sur pied, mais il n'y perdrait pas.

Enfin, si le second terme était plus grand que le premier, l'avantage du retard dans l'abatage de l'arbre serait évident.

Deuxième hypothèse. - Pour un arbre situé dans un champ cultivé, le raisonnement qui sert à la détermination de l'exploitabilité ne diffère du précédent que parce qu'ici la situation se complique d'une circonstance nouvelle : le préjudice causé aux cultures arables, préjudice qui s'agrandit chaque année. et qui peut se calculer d'après le loyer ou le fermage, le profit net enfin, du fonds de terre que le 
développement des branches ou des racines de l'arbre enlève successivement à la culture. Ce profit net doit donc être compris dans les déductions à faire subir au prix de l'arbre afin d'en avoir la valeur nette; et par conséquent, pour qu'il ne- soit pas désavanlageux de retarder l'exploitation, il faut que la plusvalue résultant de ce retard soit équivalente, au moins, à l'intérêt de la valeur nette actuelle, augmenté de la valeur d'un jeune plant d'un an et de la somme exprimant le surcroît.de préjudice causé aux cultures.

On obtiendrait plus rapidement la solution du problème en assimilant les valeurs nettes d'un arbre isolé, aux époques successives de sa croissance, à des rentes périodiques dont on chercherait. ensuite, par les formules connues, les capitaux. Au capital le plus grand correspondrait l'exploitabilité la plus avantageuse.

\section{§3.}

De l'exploitabilité relative a la rente la plus élevée, dans son application a un massif non aménagé.

Le massif peut être soumis à une longue ou à une courte révolution; être destiné à subir des éclaircies, avant d'arriver au dernier terme de sa croissance; ou, si la révolution est très-courte, être affranchi de ces exploitations intermédiaires.

Ce dernier cas étant le plus simple, c'est celui 
dont nous nous occuperons d'abord; mais l'analogie est complète entre un massif que l'on n'éclaircit pas et un arbre isolé, et l'on devine, par suite, que les éléments à apprécier pour fixer l'âge de leur exploitabilité respective, doivent être absolument les mêmes. L'exploitabilité du massif sera donc indiquée, comme celle de l'arbre isolé, par l'âge au delà duquel la plus-value résultant du maintien du bois sur pied ne serait plus égale à l'intérêt de la valeur nette actuelle, augmenté de la valeur de la première feuille ou des feuilles ${ }^{1}$ qui se seraient accumulées, depuis l'exploitation, dans le repeuplement.

Si la révolution dépasse un certain âge, il conviendra d'effectuer des éclaircies périodiques dans le peuplement, et j'ai dit que les produits matériels de ces opérations avaient pour effet de rapprocher le terme de l'exploitabilité absolue, c'est-à-dire âu plus grand accroissement moyen. Ils auront donc aussi sur l'époque de l'exploitabilité commerciale une influence, influence qu'on appréciera en ajoutant à la valeur nette présumée de la coupe principale le prix sur pied de chacune des éclaircies précédemment effectuées, augmenté de ses intérêts pendant le temps qui se sera écoulé depuis le moment où il aura été réalisé jusqu'à celui où l'on cherche s'il serait utile de procéder à la coupe principale.

Qu'on se soit, par exemple, posé la question de

1. On appelle feuille le recru, la pousse d'une année. 
savoir s'il serait avantageux. de retarder d'une année la coupe d'un taillis de 30 ans qui aurait subi une éclaircie 10 ans auparavant : on ajoutera à la valeur nette $S$ de ce taillis de 30 ans la somme représentative du prix sur pied de l'éclaircie à 20 ans, augmenté de ses intérêts pendant 10 ans; puis, on se demandera si la plus-value que le taillis serait susceptible d'acquérir, en restant un an de plus sur pied, équivaudrait au moins à l'intérêt de la valeur $\mathrm{S}$, augmenté de la valeur de la première feuille.

Il n'y a d'embarrassant dans les calculs concernant l'exploitabilité relative à la rente la plus élevée, quand on est fixé d'ailleurs sur le taux du placement, que la valeur du jeune plant ou du jeune repeuplement destiné à remplacer l'arbre ou le massif dont l'exploitabilité est mise en question : mais en y réfléchissant avec soin, on comprendra qu'elle ne peut être exprimée exactement que par la somme qui, s'ajoutant à elle-mème, d'année en année, et croissant à intérêts, serait susceptible de reproduire la valeur nette de cet arbre ou de ce massif, à l'expiration d'une période égale à son âge actuel.

Varenne de Fenille estime que la perte résultant de la non-reproduction est égale au quotient de la valeur du peuplement exploitable par le nombre d'années de son âge. Cette manière de voir ne serait logique que si l'on faisait abstraction de l'intérèt des capitaux ; or, c'est précisément cet intérêt qui est le point d'appui de l'exploitabilité relative à la rente la plus élevée; c'est sur cet intérêt que repose la valeur 
capitale à laquelle on compare le revenu; il ne saurait être passé sous silence.

Qu'est-ce que la reproduction de la première année? C'est la première feuille, c'est le premier revenu annuel du fonds de bois. Que l'on assimile ce fonds de bois à une somme d'argent placée ou à un fonds de terre, une accumulation déterminée de ses revenus se constituera de la même manière qu'une égale accumulation des intérêts de la somme d'argent ou des rentes du fonds de terre. Choisissons celles-ci pour exemple : si le propriétaire les fait fructifier pendant $\mathbf{1 4}$ ans, quelle somme obtiendra-t-il par ces épargnes successives? - Pour le savoir, il faut ajouter à la rente de la $\mathbb{1}^{\text {re }}$ année augmentée de ses intérêts pendant 14 ans, celle de la $2^{\mathrm{e}}$ année augmentée de ses intérêts pendant $\mathbf{1 3}$ ans, celle de la $3^{\mathrm{e}}$ année augmentée de ses intérêts pendant 12 ans, ainsi que les 12 autres rentes bonifiées de leurs intérêts pendant le nombre d'années compris entre leur échéance et le terme de leur placement.

Ce qu'on fait pour le fonds de terre, on doit le faire pour le fonds de bois. La perte résultant de la non-reproduction," perte égale à la valeur de la première feuille, ne peut donc rigoureusement être représentée que par l'annuité qui, s'ajoutant successivement à elle-même et croissant à intérêts, reproduirait, dans le laps de temps indiqué par l'âge du peuplement exploitable, la valeur nette de ce peuplement.

Quant à cette valeur nette, elle s'établira en 
retranchant du prix de l'arbre sur pied : $1^{\circ}$ les frais accumulés des impôts de toute nature; $2^{\circ}$ les frais accumulés de garde, d'entretien et d'assurance, s'il y a lieu; $3^{\circ}$ les frais de repeuplement dans le cas où ils ne seraient pas mis en charge sur la vente. II n'y a pas à se préoccuper du capital plus ou moins grand que le propriétaire aurait engagé dans l'acquisition du terrain; car il ne s'agit pas ici de rechercher si un propriétaire a fait une spéculation bonne ou mauvaise en boisant une partie de sa propriété; il s'agit seulement de lui indiquer les moyens de tirer le plus grand profit des bois qu'il possède, en lui montrant à quel âge il doit les exploiter pour que leur rendement suit, par rapport à leur valeur commerciale, élevé au maximum. Je dirai, en passant, que si l'on comprenait dans le calcul les intérèts du capital engagé, on aurait pour la yaleur nette à l'époque d'exploitabilité une expression souvent nulle et quelquefois négalive, et cela s'explique, puisque cette valeur représente précisément ces intérêts.

Les déductions que j'ai énumérées sont celles qui constituent les avances obligatoires faites par le propriétaire, les avances auxquelles l'acquéreur ne pourrait pas non plus se soustraire, et sans lesquelles lạ valeur commerciale de l'immeuble ne saurait être déterminée.

Il va sans dire que ces déductions ne sont point applicables aux produits des éclaircies.

Je me suis à dessein servi de ces termes prix sur pied pour exprimer le rendement des éclaircies, au 
lieu d'employer les mots valeur nette, afin qu'on évite de retrancher de ce prix les frais d'impôt et de garde, d'entretien et d'assurance, qui ne doivent ètre déduits que de la valeur brute des coupes principales, sous peine de donner lieu à un double emploi.

Il va sans dire aussi que pour les arbres épars dans les champs cultivés, il n'y a pas à tenir compte de l'impôt foncier, puisqu'il est compris dans les déductions opérées afin de fixer le revenu net de la portion, de plus en plus grande, du terrain que le développement de ces arbres enlève successivement à la culture.

On s'attend peut-être maintenant à ce que je fasse connaître mon avis sur le taux de placement que l'on doit adopter dans les calculs qu'entraîne la fixation de l'exploitabilité commerciale. Le moment pour cela n'est point venu, et je prie mes lecteurs de suspendre leur impatience, s'ils sont assez bons pour en avoir. Nous sommes aujourd'hui en pleine théorie; n'y mêlons pas des questions de fait variables et indépendantes des principes. Le taux des placements est plus élevé en France qu'en Angleterre; il l'est moins en France qu'en Russie. La théorie que je développe s'applique à tous les pays. Je me bornerai à faire observer dès à présent, parce que c'est encore là un principe, que le taux de placement adopté pour les valeurs qu'on engage dans la production forestière, doit l'être également pour celles qu'on en dégage afin de leur donner une autre des- 
tination. Avances faites, produits réalisés, le taux de placement appliqué à l'accumulation de toutes ces valeurs ne peut être modifié, si l'on ne veut pas s'écarter du but qu'on se propose d'atteindre. Le taux des placements en bois étant de 3 pour 100 , si on allait choisir un taux plus élevé pour l'accumulation d'intérêts à laquelle on aurait à comparer la plus-value des bois laissés sur pied, on sortirait des conditions du problème; l'exploitabilité relative à la rente la plus élevée serait livrée à toutes les chances aléatoires de la spéculation et deviendrait indéterminable.

\section{\$4.}

De l'exploitabilité relative à la rente la plus élevée, dans son application à un massif aménagé.

J'ai déjà prouvé que les calculs de l'exploitabilité correspondante au plus grand accroissement moyen, soit en malière soit en argent, sont aussi bien applicables aux forêts aménagées qu'à celles qui ne le sont pas; de sorte que l'exploitabilité absolue, dans ce dernier cas, ayant été fixée, par exemple, à 120 ans, on devrait en conclure qu'une forêt aménagée, qui serait d'ailleurs placée dans des conditions semblables de végétation, fournirait chaque année le produit le plus avantageux, si elle était partagée en 120 coupes de 1 à 120 ans. Ce principe est indépendant de la nature de l'exploitabilité que l'on envisage, et je pourrais en conséquence me dispenser de rien ajouter 
à ce que j'ai dit sur la manière de procéder pour déterminer l'époque de l'exploitabilité relative à la rente la plus élevée; mais les forêts aménagées se présentent avec des caractères spéciaux qui permettent, lorsqu'il s'agit d'en obtenir le rapport le plus élevé entre le revenu net et le capital, de recourir à des moyens moins compliqués que ceux dont on se sert pour les bois non aménagés :

Soit une forêt de 100 hectares, peuplée d'une essence susceptible de rester sur pied, sans dépérir, jusqu'à 100 ans; on demande à quelle révolution il est nécessaire de l'assujettir, c'est-à-dire en combien de coupes il faut la partager, pour en retirer la plus grande rente?

Si les bois n'avaient une valeur commerciale qu'à partir de l'âge de 15, cette forêt pourrait être soulmise à 86 révolutions différentes. Choisissons parmi ces révolutions celles de 100 ans, de 75 ans, de 50 ans et de 15 ans, et cherchons quelle serait la plis avantageuse.

Avec la révolution de 100 ans, le revenu annuel se composera du produit de la coupe âgée de 100 ans et de celui des éclaircies et nettoiements dans les 99 autres coupes.

Avec la révolution de 75 ans, le revenu annuel se composera du produit de la coupe âgée de $\mathbf{7 5}$ ans et de celui des éclaircies et nettoiements dans les 74 autres coupes.

Avec la révolution de 50 ans, le revenu annuel se composera du produit de la coupe âgée de 50 ans 
et de celui des éclaircies dans les 49 autres coupes.

Avec la révolution de $\mathbf{1 5}$ ans, le revenu annuel se composera du produit de la coupe âgée de $\mathbf{1 5}$ ans et de celui des éclaircies et nettoiements dans les 14 autres coupes.

Ces produits mis de côté, il restera dans chacun des aménagements prévus un matériel sur pied plus ou moins considérable suivant l'âge de la révolution, mais indispensable pour assurer la continuité du revenu annuel, la succession non interrompue des coupes. Chaque aménagement comporte un certain rapport entre le revenu net annuel et la valeur dudit matériel sur pied. C'est ce rapport qu'il faut établir d'abord, et, cela fait, l'époque de l'exploitabilité la plus avantageuse se manifestera d'une manière évidente.

Un premier point à noter, c'est que, dans l'aménagement à 15 ans, la valeur réalisable du matériel sur pied, nécessaire pour assurer la perpétuité de la production, est nulle, puisque ce matériel comprend les 14 coupes de 1 à 14 ans, inclusivement, dont le peuplement n'a aucun prix vénal. La coupe à exploiter, la coupe âgée de 15 ans, a seule de la valeur, les éclaircies n'en ont pas. Avec cet aménagement, le rapport entre le revenu net et la valeur du matériel sur pied, permanent, est aussi grand que possible, puisqu'il est infini; l'âge de 15 ans est donc une limite inférieure au-dessous de laquelle on ne saurait descendre dans la fixation de la révolution, sous peine de renoncer à tout profit. La valeur commerciale de 
la forèt aménagée à 15 ans repose exclusivement sur le revenu; elle est égale à-la capitalisation de ce revenu au taux des placements; le revenu disparaîtrait, si l'on diminuait la révolution d'une quantité quelconque, et avec lui s'anéantirait la valeur capitale.

Une seconde remarque à faire, c'est que si, à partir de la révolution de 15 ans, on suit la marche progressive, d'un côté, du capital obtenu en multipliant le revenu net par le denier des placements, et, d'un autre côté, de la valeur du matériel șur pied que j'appellerai le capital superficiel ou réel, celui-ci, qui est nul dans l'aménagement à 15 ans, s'augmente ensuite dans une proportion plus grande que l'autre, de sorte que, après lui être resté inférieur pendant quelque temps, il devient son égal et le dépasse enfin de plus en plus. Cela se conçoit : le premier capital, que je distinguerai désormais par le mot nominal, est dans un rapport constant avec le revenu; or, le revenu ne s'accroît point, au fur et à mesure qu'on élève l'âge de la révolution, dans une aussi grande proportion que le capital superficiel, que le capital réel.

Nous ne nous éloignerons pas beaucoup de la possibilité des choses en supposant que, pour l'aménagement à 100 ans, le rappoit du revenu au capital superficiel, au capital réel, sera de $11 / 2$ pour 100 ; pour celui à 75 ans, de 2 pour 100 ; pour celui à 50 , de 3 pour $100 \ldots$; mais alors, et si l'on admet que 3 pour 100 soit le taux des placements en biens- 
fonds boisés, il est clair que l'âge de 50 ans est une limite supérieure que l'on ne pourrait dépasser dans le choix de la révolution, sans se condamner à une perte d'autant plus forte que la révolution serait plus longue.

15 et 50 ans, voilà donc les âges entre lesquels doit se renfermer la révolution pour satisfaire au but que-l'on poursuit! Si l'on veut maintenant préciser le terme intermédiaire auquel il convient de la fixer, on poussera à leur dernière conséquence les considérations sur lesquelles on s'est appuyé pour réduire la révolution de 100 à 75 ans d'abord; puis de 75 à 50 ans. Ces réductions successives pouvaient avoir il est vrai pour résultat de diminuer le revenu net de la forêt; mais elles étaient justifiées surabondamment par l'intérêt seul de la portion de la superficie dont elles permettaient la réalisation. On se disait : Puisque l'intérêt de cette portion, réalisée et placée dans les conditions désirables de solidité, sera plus grand que le revenu qu'elle me fournit sous la forme où elle est, il n'y a pas à hésiter, changeons cette forme. C'était là un raisonnement très-rationnel, d'une justesse incontestable, et qui peut se traduire ainsi : Il y a avantage à diminuer la révolution, lorsque l'amoindrissement du capital superficiel, réel, qui en estle résultat, doit être accompagné d'un amoindrissement proportionnellement moins fort du revenu net, ou, ce qui revient au même, du capital nominal, du capital obtenu par la capitalisation de ce revenu au taux des placements. Il ne pouvait y avoir doute 
à ce sujet pour les révolutions supérieures à celle de 50 ans. Pour celles qui lui seraient inférieures, l'évidence n'existe plus au même degré; mais il sera facile de la dégager si nous supposons qu'on soit fixé sur le capital superficiel, le capital réel, et le revenu net" correspondant que comporte l'aménagement à un âge quelconque. En effet, pour reconnaître s'il y a ou non opportunité à adonter une révolution plus petite que celle de 50 ans, il suffira d'ajouter à l'intérêt de la somme que l'on réaliserait en raccourcissant la révolution, le revenu net que la forêt fournirait encore après ce raccourcissement, et de comparer le total au revenu net actuel de la forêt.

Si l'âge de 50 ans était, par impossible, supérieur à celui correspondant au plus grand revenu forestier moyen, la diminution de la révolution, tout eù amoindrissant le capital superficiel, aurait pour effet d'augmenter le revenu forestier ; et, dans ce cas, celte diminution serait très-avantageuse, puisque à l'accroissement du revenu forestier s'ajouterait l'inté-

- rềt du prix de la portion du capital superficiel, du capital réel, que l'on aurait réalisée.

Si le même âge de 50 ans était inférieur à celui correspondant au plus grand revenu forestier moyen, la diminution de l'âge de la révolution entraînerait celle du revenu forestier; mais il se pourrait que la différence, entre le revenu qu'on aurait retiré jusqu'alors et celui qu'on retirerait désormais, fût plus que couverte par l'intérêt de la portion réalisée du capital superficiel; l'avantage d'une révolution plus 
courte, pour être moins grand que dans le premier cas, n'en serail pas moins certain.

Enfin, si cette différence précitée n'était pas compensée par l'intérêt de la portion réalisée du capital superficiel, ce serait une preuve que l'on ne pourrait pas baisser, sans préjudice, l'âge de l'aménagement.

En résumé, il y a, dans le raccourcissement de la révolution et dans l'amoindrissement du capital superficiel, du capilal réel qui en résulle, un point auquel on doit s'arrêter, quand on recherche l'exploitabilité relative à la rente la plus élevée, et ce point est indiqué par le moment où tout amoindrissement nouveau entraînerait une diminution proportionnellement plus forte du revenu net, et par conséquent du capital nominal.

L'exploitabilité relative au rapport le plus grand entre le revenu et le capital, est soumise à certaines lois dont plusieurs méritent d'être particulièrement signalées.

\section{\$ 5 .}

Lois auxquelles est soumise l'exploitabilité relative à la rente la plus élevée.

$1^{\circ} L^{\prime}$ exploilabilité relative à la rente la plus élevée se réalise presque toujours avant celle qui correspond au plus grand accroissement moyen; elle est plus rapprochée pour les arbres épars dans les champs cuttivés, que pour ceux qui sont situés dans les terres 
incuttes, el moins rapprochée pour les massifs qui ne sont pas assujettis à des éclaircies périodiques, que pour ceux qui le sont.

$2^{\circ}$ Elle se fait d'autant moins attendre que les essences sont de moins bonne qualité, et moins susceptiblesd'acquérir de l'utilité et de la valeur en vieillissant.

$3^{\circ}$ Enfin, et c'est le point le plus remarquable, elle est d'autant plus près de coincider avec celle du plus grand revenu moyen, que l'intérêt de l'argent est moindre; de sorte que cet intérêt étant nul, les deux exploitabilités se présenteraient à la même époque.

Consacrons quelques instants au développement de ces propositions; car nous aurons à en tirer des conséquences pratiques intéressantes.

$1^{\circ} L^{\prime}$ exploitabilité relative à la rente la plus élevée se réalise presque toujours avant celle qui correspond au plus grand accroissement moyen.

Cela provient de ce que la plus-value d'un bois, résultant de l'augmentation annuelle de la matière ligneuse, dans un temps donné, est ordinairement moindre que la somme que procureraient, dans le même temps, les intérêts d'un capital équivalent à la valeur nette de ce bois. C'est dans la phase descendante des accroissements annuels que se rencontre l'époque du plus grand accroissement moyen; or, quand on assimile un bois à une somme d'argent placée, et qu' on veut en obtenir le plus grand profit, on ne peut, sauf de très-rares exceptions, sous peire de manquer son but, prolonger la révolution au delà du terme qui marque l'apogée de la végétation. En 
effet, lorsque les bois ont dépassé cet apogée, le prix du mètre cube ne s'élève plus; la valeur de leur accroissement annuel diminue donc constamment, à partir de cette époque, avec l'accroissement lui-même, et ne saurait suppléer à l'intérêt toujours croissant du produit en argent qu'on aurait réalisé par l'exploitation.

On se convaincra également, sans peine, que l'exploitabilité relative à la rente la plus élevée, est plus rapprochée pour les arbres épars dans lés champs cultivés, que pour ceux qui sont situés dans des terrains vagues, si on se rappelle que pour qu'il y ait avantage à retarder la coupe dans ce dernier cas, il suffit que la plus-value présumée de l'accroissement soit plus grande que l'intérêt de la valeur de l'arbre, pendant un an, augmenté.du prix de la feuille; tandis que dans le premier cas, à ces éléments il faut en ajouter un autre qui augmente chaque année, et qui consiste dans le préjudice causé à la culture arable par le développement, soit des branches, soit des racines de l'arbre. L'accroissement de cet arbre étant supposé le même dans les deux cas, il est évident qu'il atteindra plus tôt dans le premier que dans le second, la limite au delà de laquelle il cesserait d'être rémunérateur.

C'est sans doute à cette raison que tient la défaveur dont les plantations, dans les champs cultivés, sont l'objet de la part de nos agronomes les plus renommés, MN. Dombasle, Gasparin, etc. ${ }^{1}$.

1. On applique souvent aux forêts jardinées dont ou veut régler 
Enfin, l'exploitabilité relative à la rente la plus élevée, est plus tardive pour les masssifs éclaircis périodiquement, que pour ceus qui ne le sont pas. Cela s'explique par les produits intermédiaires que fournissent les premiers; produits qui, en augmentant tout à la fois le revenu net et le capital obtenu pár la capitalisation de ce revenu au taux des placements, tendent à reculer l'époque où ce capital devient moindre que le capital superficiel.

$2^{\circ} L^{\prime}$ exploilabitité relalive à la rente la plus élevée est d'autant plus rapprochée, que les essences sont de moins bonne qualité et moins susceptibles d'acquérir de l'utilité et de la valeur en vieillissant.

Ainsi, pour des bois blancs qui ne seraient propres qu'au chauffage, l'époque de la coupe devrait être plus avancée que pour des bois durs qui, propres au chauffage seulement, dans les premières années de leur existence, ne tardent pas à acquérir des qualités précieuses qu'on recherche pour une foule d'ouvrages, et qu'on paye en conséquence.

Si l'on veut saisir la vérité de cette proposition,

l'exploitabilité, le procédé des expériences individuelles, c'est-àdire qu'on fait entrer les arbres dont elles se composent dans la catégorie des arbres isolés. Ce procédé n'est pas, à beaucoup près, aussi exact que celui qui consisterait à évaluer le revenu moyen annuel et à le comparer au capital superficiel. Dans tous les cas, si on y a recours, il ne faut pas oublier de tenir compte du dommage causé aux sujets environnants par l'arbre d'expérience; ce faisant, on reconnaîtra, comme la culture l'enseigne d'ailleurs, que la méthode jardinatoire est de toutes la moins produrtive. 
il est encore nécessaire de se rappeler qu'il est avantageux seulement de relarder l'exploitation, quand l'on est autorisé à espérer que l'intérêt du produit réalisable, joint à la valeur de la première feuille, trouvera une compensation suffisante dans la plus-value résultant de l'accroissement.

Négligeons, pour simplifier la question, la valeur de la première feuille, qui ne saurait exercer une influence importante sur la solution du problème, et dont l'absence est même de nature à corroborer notre démonstration. Ne mettons dans la balance que l'intérêt du produit réalisable, d'une part, et, de l'autre, la plus-value résultant de l'accroissement. Supposons enfin que le prix du bois reste le même, quel que soit l'âge.

N'est-il pas vrai qu'en suivant un arbre ou un massif dans ses développements annuels successifs, on observe que le sacrifice de l'intérêt auquel on se soumet, en retardant l'exploitation, est proportionnel à la somme des accroissements antérieurs; tandis que la plus-value résultant de l'accroissement, au contraire, n'est jamais fonction que d'une année? Progressive d'abord, elle devient ensuite stationnaire, puis de plus en plus petite. En présence d'un préjudice, d'une perte, qui s'augmente en quelque sorte dans une progression géométrique, se trouve donc un avantage variable dans des limites nécessairement étroites. Admettons, au reste, un instant, que l'accroissement annuel soit constant et représenté par 1, hypothèse d'autant plus acceptable que 
l'erreur qu'elle renferme affecte également les deux termes de notre comparaison. L'arbre ou le massif qui vaudra 1 à 1 an, 2 à 2 ans, vaudra 100 à 100 ans; la plus-value annuelle sera toujours égale à l'unité, tandis que l'intérêt du produit réalisable, sera pour le massif de 100 ans, cent fois plus fort que pour celui de 1 an.

Mais si nous supposons actuellement que le prix du mètre cube grandit avec l'âge, il est incontestable que l'écart entre les deux facteurs de la comparaison en sera atténué. Pour que cet écart disparût complétement, il faudrait que le prix du mètre cube grandit dans une progression semblable à celle que suivrait l'intérêt des valeurs successivement accumulées, ou, en d'autres termes, que le prix du mètre cube à 100 ans fùt cent fois plus élevé que celui du mètre cube à 1 an.

En fait, la progression que suit le prix des bois selon leur âge et leurs dimensions, est fort éloignée de celle qui aboutirait au résultat que procure l'accumulation des intérêts.

Ce que je viens de dire peut encore, et en deux mots, se démontrer de la manière suivante :

$\mathrm{Au}$ lieu de considérer l'accroissement annuel, abstraction faite des accroissements précédents représentés par le bois sur pied, considérons-le dans son rapport avec ces accroissements.

Soit $\mathrm{V}$ le volume de l'arbre, $a$ son accroissement annuel, $n$ son âge. Pour connaitre le rapport à tant pour 100 , on posera 
$\mathrm{V}: a:: 100: x ; x=\frac{a \times 100}{\mathrm{~V}}$; mais $a$ (nous supposons l'accroissement annuel constan $t)=\frac{\mathrm{V}}{n} ;$ on a donc $x=\frac{100 \mathrm{~V}}{\mathrm{~V} n}=\frac{100}{n}$, de sorte que lorsque $n$ sera $>100$, le rapport sera $<1$; or, le prix du bois ne changeant pas avec l'âge, l'intérêt qu'on obtiendrait de la réalisotion du prix de l'arbre suivrait une progression semblable, mais en sens inverse, c'est-à-dire cruissante : à 100 ans l'intérêt de V serait $\frac{100}{1}$.

Donc, l'équilibre entre la plus-value de l'arbre laissé sur pied et les intérêts qu'on. retirerait de sa valeur, ne pourrait être établi, que si l'augmentation du prix de cet arbre était suffisante, pour racheter la diminution du rapport de l'accroissement annuel à la somme des accroissements antérieurs.

L'échelle des prix, telle qu'elle est aujourd'hui fixée par les transactions commerciales, ne saurait réaliser la compensation désirable; mais comme elle exerce néanmoins une très-grande influence sur la fixation de l'exploitabilité commerciale, on commettrait de graves erreurs, si on ne la consultait pas avec soin. La plus-value résultant des qualités plus précicuses qu'acquiert le bois en vieillissant, n'est pas uniforme dans sa progression; elle éprouve dès temps d'arrêt plus ou moins prolongés șuivant les essences et les convenances industrielles ou commerciales; puis, elle augmente brusquement. C'est une circonstance à laquelle il importe de faire beaucoup d'attention. Si les calculs relatifs à la recherche de l'âge 
d'exploitabilité s'appliquaient par exemple à un de ces temps d'arrêt, ils pourraient conduire souvent à hâter l'exploitation; tandis qu'en prenant en considération l'époque plus ou moins prochaine où le bois serait devenu propre à une destination supérieure, on aurait peut-être trouvé dans les avantages de cette destination, une compensation plus que suffisante pour la perte résultant du retard de l'exploitation.

Citons un exemple : Les sapins qui ont moins de $0^{\mathrm{m}}, \mathbf{1 6}$ de diamètre au gros bout, ne sont guère propres qu'au chauffage, et s'ils ne devaient pas, en grossissant, acquérir, toutes proportions gardées, un plus grand prix, il est présumable qu'on ne les laisserait pas dépasser cette dimension; mais audessus de $0^{\mathrm{m}}, 16$ et jusqu'à $0^{\mathrm{m}}, 22$ de diamètre, ils prennent la qualité de chevrons, trouvent ainsi leur emploi dans les constructions, et empruntent à cette destination une valeur relativement plus élevée; plus tard, quand ils dépassent $0^{\mathrm{m}}, 22$, ils deviennent propres à faire des pannes simples, c'est-à-dire, des pièces plus recherchées, plus utiles que les premières et par conséquent, à volume égal, plus chères; ils demeurent dans cette catégorie jusqu'à ce que leur diamètre atteigne $0^{\mathrm{nt}}, 32$. De $0^{\mathrm{m}}, 32$ jusqu'à $0^{\mathrm{m}}, 36$, ils sont comptés comme pannes doubles, c'est-à-dire, comme bois de grosse charpente, mais ils n'ont pas encore atteint l'apogée de leur prix. Au-dessus de $0^{\text {in }}, 36$, ils servent au sciage, et c'est alors seulement que leur maximum de valeur et d'utilité est obtenu. 
Or, pour les conduire jusque-là, il est nécessaire de les laisser sur pied jusqu'à 80 ans environ': C'est ce que font les propriétaires des Vosges; ils trouvent dans l'augmentation du prix du bois, suivant les dimensions, un dédommagement, sinon une compensation, à la diminution du rapport entre le revenu annuel èt le capital engagé.

Je répéterai encore que tout ce que j'ai à dire sur l'exploitabilité forestière, est applicable aussi bien aux bois aménagés qu'à ceux qui ne le sont pas, et, lorsque je néglige de choisir mes preuves dans les deux catégories, c'est que la démonstration ne me paraît pas en avoir besoin. Mes observations relatives à l'échelle des prix me paraissent, cependant, tellement importantes, qu'au risque d'être accusé de rebattre toujours la même question et d'entrer dans des développements superflus, j"en vérifierai la justesse dans une forêt aménagée.

Soit une forêt régulière exploitable dans une révolution de 25 ans : la coupe de 25 ans formera le revenu; le peuplement des 24 autres coupes constituera le capital superficiel. Si nous supposons que le bois ne soit, jusqu'à 25 ans, bon que pour le chauffàge, mais qu'à 26 ans, il devienne propre à une destination supérieure, qu'en devra-t-on conclure? - On en devra conclure que, selon toute probabilité, il serait avantageux d'augmenter d'un an l'âge de la révolution et de partager la forêt en 26 coupes au lieu de 25. Je dis, selon toute probabilité, car cette destination supérieure, qui ferait attribuer au bois 
un prix plus élevé, n'affecterait nullement le capital superficiel, lequel resterait toujours propre au chauffage seulement, et elle porterait tout entière sur le revenu net annuel; d'où il résulterait que le rapport de ce revenu au capital superficiel serait augmenté.

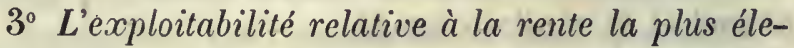
vée, est d'autant plus près de coïncider avec l'époque du plus grand revenu.moyen, que l'intérêt des placements en argent est moindre.

C'est une proposition que les explications précédentes ont rendue évidente ; cependant, si quelquesuns de mes lecteurs n'étaient pas de cet avis, je les prierais de me prêter un moment d'attention.

On se souvient que l'exploitabilité qui procure le plus grand revenu moyen, est indiquée par l'année. où l'accroissement annuel devient égal, en valeur, à l'accroissement moyen. On n'a pas oublié non plus que, pour fixer l'âge qui correspond à l'exploitabilité commerciale, il faut rechercher l'année à l'expiration de laquelle la plus-value, résultant de l'accroissement, serait équivalente à l'intérêt des accroissements antérieurs, augmenté de la valeur de la première feuille, c'est-à-dire de la somme qui, s'ajoutant à elle-même d'année en année, et croissant à intérêts, serait susceptible de reproduire, dans le même temps, la valeur nette de ces accroissements. Il y a là une équation dont le premier membre ne présente qu'un terme : la plus-value résultant de l'accroissement; dont le second membre se compose 
de deux termes : l'intérêt des accroissements antérieurs et la première feuille. Si l'intérêt de l'argent était nul, le premier membre ne changerait pas; dans le second, le premier terme (intérêt des accroissements) disparaitrait, et il ne resterait que la première feuille; mais, cette première feuille qui ne pourrait plus s'accroître à intérêt, deviendrait tout simplement égale à la somme qui, s'ajoutant à ellemême, d'année en année, pendant la révolution, représenterait à la fin de cette révolution la valeur nette du bois exploitable.

La révolution étant exprimée par $n$, et la valeur nette du bois exploilable par $\boldsymbol{P}$, la feuille serait égale à $\frac{P}{n}$, c'est-à-dire au revenu moyen. L'exploi . tabilité commerciale serait, en conséquence, indiquée par l'âge où la plus-value résultant de l'accroissement, plus-value qui n'est autre chose que la valeur de l'accroissement annuel, deviendrait égale au revenu moyen.

On voit clairement, par là, que l'intérêt de l'argent étant réduit à 0 , l'exploitabilité commerciale se confond avec celle qui correspond au plus grand revenu moyen absolu.

La démonstration algébrique de ce théorème est d'ailleurs bien simple, et je vais la donner, quoique ces sortes de preuves soient loin de jouir aujourd'hui d'une grande faveur.

Appelons : 
$n$, l'âge du bois dont on se demande s'il est bon de retarder l'exploitation;

$a$, la valeur de l'accroissement à la $n+1^{\mathrm{e}}$ année;

A, la valeur des accroissements antérieurs;

ఢ, la première feuille;

$r$, l'intérêt de la somme qu'on réaliserait en exploitant actuellement.

Pour qu'il ne soit pas avantageux de retarder l'exploitation, il faut que l'on ait :

$$
\text { (1) } r=\mathrm{A} a+\varphi
$$

Cherchons la valeur de $\varphi$.

Cette valeur est celle qui, s'ajoutant à elle-même d'année en année, et croissant à intérêts, reproduirait, à l'expiration de ces $n$ années, la valeur $\mathbf{A}$ : nous avons donc (avec les intérêts composés):

$$
\begin{gathered}
\mathrm{A}=\varphi(1+r)^{n-1}+\varphi(1+r)^{n-2} \cdots \ldots+\varphi=\varphi\left[(1+r)^{n-1}\right. \\
\left.+(1+r)^{n-2}+\ldots(1+r)+1\right] \\
\text { d'où } \varphi=\frac{\mathrm{A}}{(1+r)^{n-1}+(1+r)^{n-2}+\ldots(1+r)+1 .}
\end{gathered}
$$

Remplaçant 9 par sa valeur dans l'équation(1), nous avons :

$$
a=\mathrm{A} r+\frac{\mathrm{A}}{(1+r)^{n-1}+(1+r)^{n-2}+(1+r)+1}
$$

Sans pousser plus loin la réduction, on voit que si $r=0$, A $r$ disparait, et le dénominateur du $2^{\circ}$ terme du second 
membre de l'équation devient égal à $n$, c'est-à-dire à autant de fnis l'unité qu'il y a d'années dans l'âge du bois.

$$
\text { On a donc : } a=\frac{\mathrm{A}}{n}
$$

C'est, par conséquent, lorsque la valeur de l'accroissement annuel est égale au revenu moyen des années antérieures, que l'exploitabilité commerciale se réalise, l'intérêt étant nul; mais le revenu moyen est maximum; quand il est égal au revenu annuel; donc, etc.; etc.

Si les calculs se faisaient aux intérêts simples, on arriverait au même résultat; car $\varphi$ deviendrait alors égal à

$$
\frac{\mathrm{A}}{1+(n-1) r+1+(n-2) r+\ldots 1+r+1}
$$

Et l'on a :

$a=\mathrm{A} r+\frac{\mathrm{A}}{1+(n-1) r+1+(n-2) r+\ldots 1+r+1}$

équation qui se transforme encore, quand $r=0$, en celle-ci : $a=\frac{A}{n}$.

Les forêts aménagées offrent mieux que les autres, les moyens de vérifier l'influence de l'abaissement de l'intérêt sur la fixation du terme de l'exploitabilité relative à la rente la plus élevée. La seule circonstance qui soit de nature à engager les propriétaires à raccourcir la durée de la révolution, consiste, en effet, dans lá possibilité de placer avantageusement, à intérêt, la somme qu'ils réaliseraient en diminuant 
le capital superficiel; mais si cette possibilité leur était enlevée, ils ne se laisseraient évidemment guider dans la recherche de l'exploitabilité, que par le désir de retirer de leurs propriétés le revenu moyen absolu le plus considérable. J'ai montré, d'un autre côté, que c'est surtout à la supériorité que le capital superficiel, le capital réel, présente souvent sur le capital obtenu en multipliant le revenu par le denier du placement, que l'on juge de l'opportunité et des avantages du raccourcissement de la révolution; or cette supériorité ne se présenterait jamais si l'intérêt de l'argent était infiniment petit, puisque alors les valeurs capitales deviendraient infiniment grandes.

La première partie de mon travail est terminée. Nous allons quitter le domaine de l'absolu, de la théorie pure, de l'abstraction, pour entrer dans celui de la vérité relative, de la pratique, de la possibilité des choses. On doit prêvoir que, dans cette nouvelle voie, nous serons obligés de nous écarter plus ou moins de la ligne de conduite que nous aurions à suivre pour obéir rigoureusement aux principes qui ont été développés. L'essentiel est que nous ne les perdions jamais de vue : ces principes, je l'espère, n'en resteront pas moins recommandables. Que dirait-on d'un voyageur qui, surpris par l'obscurité, s'aviserait de mépriser la lumière lointaine qui lui indique le but à atteindre, parce que les accidents du terrain l'empêcheraient de prendre, pour y arriver, la route la plus directe? - On douterait de son bon sens. Semblables à ce voyageur seraient ceux qui me 
reprocheraient le temps que j'ai consacré à la démonstration de certaines lois, dont les prescriptions ne pourraient être intégralement et rigoureusement appliquées.

Parmi les circonstances qui nous forceront de modifier dans l'application les règles que nous avons posées, les plus importantes, peut-être, sont celles qui résident dans les conditions d'existence de l'homme, et particulièrement dans la courte durée de cette existence. Il en résulte que l'application d'un principe, d'une loi, d'une règle de conduite, bonne pour un temps donné, ne l'est plus quand on le dépasse. Les mours, la législation, la constitution de la propriété, exercent à cet égard une grande influence : elles peuvent plus ou moins reculer l'époque au delà de laquelle les appréciations humaines cessent de présenter un caractère suffisañ de probabilité, et tombent dans le domaine des futurs contingents.

Je me borne aujourd'hui à indiquer celte idée, à laquelle $\mathrm{j}$ 'aurai souvent occasion de revenir. 


\section{CHAPITRE DEUXIEME.}

DE L'EXPLOITABILITÉ DANS SES RAPPORTS

AVEC LES EXIGENCES

DE LA VÉGÉTATION ET DE LA CULTURE.

Nous avons raisonné, jusqu'ici, sur l'exploitabilité, comme si rien ne s'opposait à ce qu'elle pût être fixée à l'une quelconque des années comprises dans la durée de la vie végétale.

Cette hypothèse n'était pas vraie.

Il y a dans la nature, des choses, des exigences, qui sont indépendantes de la volonté de l'homme, et qui renferment le choix de l'âge d'exploitabilité dans des limites plus étroites.

L'un des principaux buts que l'on poursuit en sylviculture est, personne ne l'ignore, 'la régénération naturelle.

Toute méthode d'exploitation des forêts, disent les auteur du Cours de Culuure des Bois, doit satisfaire aux deux conditions fondamentales suivantes:

$1^{\circ}$ Régler la quotité des coupes annuelles de manière à procurer un rapport soutenu.

$2^{\circ}$ Assurer, par ces coupes mêmes, la régénération naiurelle.

Or, les bois.ne se régénèrent naturellement que 
par les semences ou par les souches ${ }^{1}$; ils ne portent de semences fertiles qu'à un certain âge, qui varie selon les espèces, mais qui est au moins de cinquante à soixante ans; ils cessent de repousser par les souches à un âge également variable, mais qui ne dépasse guère quarante ans; enfin, il y en a qui se refusent absolument à ce dernier mode de reproduction.

De là on est amené à conclure, qu'avant de fixer l'âge d'exploitabilité d'un massif, la première chose à faire est de choisir le mode d'exploitation applicable à ce massif.

\section{ARTIGLE PREMIER.}

CHOIX DU MODE D'BXPLOITATIOS.

Ce choix ne saurait être douteux pour les essences qui sont privées de la propriété de repousser par la souche. Il est évident que le mode de la futaie est le seul qui leur soit applicable; mais les bois feuillus jouissent tous, à un degré plus ou moins grand, de la faculté de produire des rejets quand on les a coupés par le pied; ils peuvent par conséquent être exploités soit en taillis, soit en futaie; et suivant qu'ils seront exploités d'après l'un ou l'autre mode, leur exploitabilité devra être ou très-avancée ou trèsreculée.

1. Je ne tiendrai pas compte des drageons et des marcoltes qui ne sont que des exceptions. 
Les considérations d'après lesquelles on se guide, dans le choix du mode d'exploitation, sont comme toujours, en économie forestière, de deux sortes : culturales et économiques.

Th Je vais comparer, à ces deux points de vue, le taillis simple à la futaie, et je m'occuperai ensuite du taillis composé.

\section{$\$ 1^{\text {er. }}$}

Du taillis simple et de la futaie au point de vue cultural.

M. Parade a parfaitement démontré, dans son examen comparé des trois principales méthodes d'exploitation, les inconvénients du taillis et les avantages de la futaie. Je ne veux pas refaire cet examen. Je me bornerai à mettre en relief les principales conséquences qui en découlent, et à les accompagner de quelques réflexions.

Ces conséquences se résument dans les quatre propositions suivantes :

$1^{\circ}$ La méthode de la futaie a pour résultal d'améliorer le sol; celle du taillis tend à le détériorer.

$2^{\circ}$ La méthode de la futaie permet de garantir la végétation contre les intempéries du climal; celle du taillis la livre sans défense à ces intempéries.

$3^{\circ}$ La méthode de la futaie est beaucoup plus favorable à la régénération que celle du taillis.

$4^{\circ}$ La méthode de la futaie favorise la croissance des sujets qui, composent un massif; celle du taillis l'entrave. 
$1^{\circ}$ Influence du mode d'exploitation sur le sol. .

Le sol, pour la culture des bois, emprunte surtout ses éléments de fertilité à l'humus. Il peut à la rigueur se passer d'humus, quand il est constitué physiquement d'une façon particulièrement avantageuse. Il n'en a jamais trop et il devient stérile quand, à la mauvaise qualité de ses composants minéralogiques, il joint l'absence complète de substances organiques.

Mais l'humus ne se forme, on le sait, que sous l'influence de l'humidité, de la chaleur et d'un air calme. Cette influence est grande et prolongée dans les futaies; elle est faible et courte, au contraire, dans les taillis dont le sol est, à des périodes rapprochées, exposé à l'action desséchante du soleil et des rents.

Avec le temps, le sol s'enrichit donc de plus en plus dans le premier cas; avec le lemps, il s'appauvrit donc de plus en plus dans le second.

Je crois que cet appauvrissement n'est plus contesté aujourd'lini par personne; il devient plus ou moins grave suivant le climat, la situation, et la nature de la base minéralogique.

Si le climat est humide, les inconvénients du taillis seront moindres quant à la fertilité du sol, que s'il est sec; si le terrain est en plaine, moindres que s'il est en pente; s'il est en pente septentrionale, moindres que s'il est en pente méridionale; s'il est imperméable, moindres que s'il ne l'est pas ${ }^{1}$.

4. On pourrait fournir de no.nbreuses preuves à l'appui de 
- $2^{\circ}$ Influence du mode d'exploitation sur l'action des intempéries.

La possibilité de défendre les bois contre les. intempéries dépend, jusqu'à un certain point, de la durée de la révolution, en ce sens que plus cette dernière est longue, plus puissante est la barrière opposée par l'application des règles d'assiette, soit aux vents,

ces propositions, si cela était nécessaire. C'est le mode d'exploitation en taillis qui a amené en France la dégradation d'une grande partie du sol forestier, dégradation à laquelle on ne peut obvier aujourd'hui qu'en cultivant des pins sylvestres dans des terrains où le chêne prospérait autrefois. C'est ce mode qui a dépeuplé la forêt d'Orléans, celle' de Fontainebleau, celle de Saint-Germain, celle de Compiègne; forêts dont le sol sablonneux aurait eu besoin d'un couvert épais et constant. Je cite ces forêts. parce que tout le monde les connait au moins de nom, qu'elles sont à la porte de la capitale, et qu'il est facile d'aller y vérifier l'exactitude de mes assertions. J'ai d'ailleurs mes cartons remplis de notes qui ont été recueillies dans d'autres forêts, et qui constatent les mèmes faits; mais je ne crois pas que la ruineuse influence du taillis sur le sol soit nulle part aussi évidente, aussi - palpable, que dans une forêt des environs de Clermont (Oise), la forêt de Hez. Cette forêt appartient, par portions à peu près égales, à l'État et à la famille d'Orléans. La portion de l'État se compose d'une très-belle futaie de hêtres et de chènes; la portion de la famille d'Orléans se compose, au contraire, d'un taillis dont la consistance et la végétation sont souvent plus que médiocres. Les deux portions sont pourtant enchevêtrées l'une dans l'autre, et leur sol, identique quant aux éléments minéralogiques, est formé d'une mince couche de sable reposant sur un banc impénétrable aux racines; partout, à côté de la croissance rapide dela futaie qui annonce un sol fertile, on est frappé de la croissance languissante du taillis qui accuse un sol appauvri, et tandis que la futaie ne renferme que des bois durs, le taillis est déjà rempli de bois blancs et de morts-bois. 
soit aux autres météores. Les futaies, par la hauteur et la consistance des massifs qu'elles renferment, offrent pour les parties d'une forêt qui ont besoin d'être abritées, une protection bien autrement efficace que les taillis.

Les procédés d'exploitation que comportent les futaies permeltent, en outre, de ne livrer le jeune repeuplement aux influences de l'atmosphère, que lorsqu'il est assez robuste pour les supporter; tandis que, dans les taillis, rien ne garantit le recru contre les effets de la sécheresse ou de la gelée.

Depuis leur naissance jusqu'au terme fixé pour leur abatage, les massifs traités en futaie sont maintenus dans un état serré et peuvent, par l'appui mutuel que se prêtent les brins dont ils se composent, supporter le poids du verglas et des neiges. Il n'en est pas ainsi pour les taillis qui sont formés de cépées irrégulièrement espacées. Aussi, le verglas et les neiges y causent-ils de grands et fréquents dommages.

$3^{\circ}$ Influence du móde d'exploitation sur la régénération.

II n'y a de régénération complète et véritable que celle qui s'opère par les semences ${ }^{1}$.

Dans ce cas, le plan nouveau, qui est le produit de la régénération, réunit toutes les conditions nécessaires à un développement normal; il est doứ d'une virtualité propre, distincte, indépendante de celle

1. Yoir la note page 127. 
qui lui a donné naissance. L'arbre qu'on coupe par le pied, et qui repousse, ne produit pas des êtres nouveaux, il continue de vivre. Après un certain nombre d'exploitations, sa vie s'éteignant, il ne donnera plus de rejets. Celui qui se régénère par la semence peut, au contraire, se régénérer ainsi éternellement. La futaie a donc sur le taillis un avantage considérable au point de vue de la perpétuation de l'espèce.

D'un autre côté, quelque favorables que soient les conditions de régénération dans lesquelles un massif se trouve placé, cette régénération n'en est pas moins une crise très-chanceuse dont il importe de retarder autant que possible le retour. Une futaie, dans laquelle elle ne se présentera qu'une fois par siècle, sera bien préférable, à ce nouveau point de vue, à un taillis.

Enfin, c'est dans leur jeunesse que les bois sont re plus exposés à être envahis par les essences secondaires et les morts-bois. Dans les taillis, les dangers de cet envahissement sont beaucoup plus redoutables que dans les futaies; non-seulement par le motif que je viens d'indiquer, c'est-à-dire à cause de la fréquence plus grande des époques de régénération; mais aussi parce que, quelque complet que soit un taillis à l'époque de son exploitabilité, il présente de nombreux vides aussitôt après son exploitation.

$4^{\circ}$ Influence du mode d'exploitation sur la croissance.

Pour qu'un massif végète dans les conditions les 
plus favorables, il faut que les sujels dont il se compose soient espacés régulièrement et de manière ì participer également aux influences de l'atmosphère. Il est possible de réaliser ces conditions dans un massif de futaie formé de brins de semence. Cela n'est pas possible dans un taillis où les rejets sont disposés nécessairement par groupes, et en constituent autant qu'il y a de souches dans le peuplement; ces groupes plus ou moins distants les uns des autres, suivant que la durée de la révolution est plus ou moins longue, s'étalent inévitablement, au début de leur croissance, pour remplir les vides qui les séparent : trop serrés sur certains points, trop espacés sur d'autres, tels sont les inconvénients inévitables que présentent les massifs crus sur souches. - leur croissance en souffre et la qualité du bois sen ressent.

Sous le rapport cultural, le mode d'exploitation en taillis est donc un mauvais mode, et s'il n'y avait que les convenances culturales à consulter en sylviculture, il faudrait le rejeter sans hésitation; mais ce mode, quelque vicieux qu'il soit, est encore, à force de soins coûteux, conciliable avec les strictes exigences de la conservation des massifs, et s'il se recommandait par des motifs économiques, il pourrait être permis de l'adopter. 


\section{$\$ 2$}

Du taillis simple et de la futaie au point de vue économıque.

Les considérations économiques applicables à l'exploitation des bois sont tirées, nous l'avons vu, soit de la quantité des produits, soit de leur utilité, soit de leur valeur absolue, soit de leur valeur relative.

Cómme quantité, c'est une vérité traditionnelle que les taillis ne donnent pas ce que donnent les futaies. Toutefois, des expériences très-concluantes manquent à cet égard. La reproduction par rejets modifie profondément les phases de la végétation : elle rend celle-ci beaucoup plus active dans la jeunesse, à ce point qu'un rejet de vingt ans, par exemple, toutes conditions égales d'ailleurs, est de moitié au moins plus volumineux qu'un brin du même âge. Une conséquence de ces modifications, c'est que l'époque du plus grand accroissement moyen sê présente beaucoup plus tôt dans les taillis que dans les futaies. Un taillis est-il susceptible d'un accroissement moyen plus considérable qu'une futaie placée dans les mêmes conditions de végétation? - Telle est la question que l'on n'a pas résolue rigoureusement, qu'il faudrait résoudre, et sur laquelle pourtant il ne s'est jamais élevé le moindre doute : c'est que jusqu'à présent le maximum de la production, par hectare, que l'on a constaté dans les taillis, ne 
dépasse pas six stères, tandis que dans les futaies il s'est élevé jusqu'à douze et quinze stères.

Comme utilité, la supériorité de la futaie ne se discute pas; nous avons vu en effet que sauf de rares exceptions, l'utilité des.bois est généralement proportionnelle aux dimensions qu'ils présentent, attendu que plus ces dimensions sont fortes, plus grand est le nombre des usages divers auxquels ils peuvent servir. Ajoutons que dans les futaies, les produits accessoires, tels par exemple que ceux qu'on retire du panage, de la glandée, du pâturage, sont plus nombreux et plus importants que dans les taillis.

Comme valeur absolue, le produit du taillis étant probablement moins considérable et certainement moins utile, doit nécessairement être beaucoup moins précieux que celui de la futaie.

Comme valeur relative, au contraire, le rapport entre le revenu et le capital superficịel étant d'autant moins grand que la révolution est plus longue, il est évident que le taillis peut fournir un produit plus avantageux que la futaie, puisqu'il comporte une révolution beaucoup plus courte.

$\$ 3$.

Conclusion des deux paragraphes précédents.

Ainsi, parmi les considérations culturales et économiques que l'on doit interroger, quand il s'agit 
d'arrêter le mode d'exploitation applicable à une forêt, il n'y. en a qu'une, celle de la rente, qui puisse faire préférer le taillis à la futaie.

Je vais examinel maintenant jusqu'à quel point la réserve, dans les taillis, d'un nombre plus ou moins grand de baliveaux, est de nature à modifier cette conclusion; je vais examiner quelle est l'influence de ces baliveaux, tant sous le rapport cultural que sous le rapport économique.

\section{$\$ 4$.}

Des réserves dans les taillis.

Pour tous ceux qui ne jugent des choses qu'au cabinet, le taillis sous futaie est un mode d'exploitation fort ingénieux et très-avantageux, parce qu'il parait susceptible de se plier à toutes les convenances de l'exploitabilité. Si on recherche, par exemple, la rente la plus élevée, on la réalisera au moyen du sous-bois; tandis qu'on pourra, au moyen des futaies exploitables à des âges différents, rechercher et réaliser tantôt les produits les. plus utiles, tantôt les plus considérables, élever ici des essences d'une grande longévité et à côté des espèces de courte durée. Voilà ce que disent les gens superficiels; mais ceux qui ont étudié les choses de près ne sont pas du même avis, et voici ce qu'ils pensent des baliveaux sur taillis : 
La première condition, la condition essentielle afin que les arbres forestiers se portent bien, c'est qu'ils soient en massif, surtout dans leur jeunesse. Dès qu'on isole ces arbres, on les place dans un état anormal dont ils soufirent beaucoup, à moins qu'on ne leur donne des soins particuliers et très-coûteux. Mais si l'isolement est défavorable aux arbres, en principe général, et lorsqu'il est permanent, il leur devient bien plus dommageable encore lorsqu'il est intermittent. Isoler un arbre, c'est comme si on le transportait d'un climat dans un autre. En tout état de cause, ce changement ne peut que lui être nuisible; il le sera d'autant plus qu'il s'effectuera à un âge moins avancé.

En fait, les baliveaux qu'on réserve dans les taillis, privés subitement de l'appui de leurs semblables; sont souvent inhabiles à se supporter. Ils tombent au moindre vent; c'est le premier danger qui les menace. Ensuite, quand ces baliveaux sont, du pied à la tète, exposés à l'influence de la lumière, ils se couvrent de bourgeons, lesquels absorbent une partie de la séve au préjudice de la cime qui se dessèche en tout ou en partie. Enfin, cette écorce, qui jusqu'alors avait pris son développement à l'ombre, a une consistance lâche; mise brusquement à découvert, elle est facilement endommagée, soit par la gelée, soit par le soleil, ce qui occasionne ainsi des vices intérieurs qui rendent les arbres plus ou moins impropres à une destination industrielle. Voilà pour ce qui regarde les baliveaux eux- 
mêmes; pour ce qui est de leur influence sur le taillis, le raisonnement et l'expérience prouvent qu'ils sont loin de compenser les inconvénients qu'on vient de leur reconnaîtré.

Buffon a observé que la gelée faisait beaucoup de tort aux taillis surmontés de nombreuses réserves. Il a constaté que, toutes circonstances égales d'ailleurs, un taillis privé de réserves en avait distancé un autre qui ne l'était pas, de cinq ans sur douze, par suite de la gelée qui avait endommagé ce dernier.

Un fait plus facile à expliquer, c'est qu'en entrỉavant l'effet de la lumière, les baliveaux, quand ils sont trop nombreux ou trop gros, nuisent nécessairement au développement du taillis; le vide se fait donc peu à peu autour de leur tronc, et quand on les abat, ils occasionnent de grands vides qu'il faut repeupler artificiellement.

On pourrait croire que par les semences qu'ils fournissent, les baliveaux contribuent au moins à la régénération du taillis. Il n'en est rien, et cela se comprend : les jeunes plants produits par leurs semences, exposés à toutes les intempéries, s'ils naissent au moment de l'exploitation, perdus dans l'obscurité dans le cas contraire, sont exposés à mourir soit par l'excès, soit par l'insuffisance de la lumière. C'est à cette dernière cause qu'il faut attribuer la disparition des chênes dans nos taillis sous futaie; disparition constatée dans tous les pays sans exception. 
D'après ce que nous venons de voir de l'influence des réserves sous le rapport cultural, -il est permis d'affirmer qu'il ne peut pas y avoir une grande différence entre le rendement des taillis composés et celui des taillis simples, lorsqu'on ne considère que la quantité de matière produite annuellement; mais les taillis composés fournissent sans contredit des produits plus utiles et par conséquent plus précieux, que les taillis simples, et, sous ce rapport, ils offrent un avantage, ils ont un mérite qui n'est point à dédaigner. On estime que dans un taillis soumis au balivage normal, la futaie entre environ pour un tiers ou un quart dans le produit total de l'exploitation, et que la moitié de cette futaie est propre à l'industrie. Ces chiffres, que je crois plutôt au-dessus qu'au-desous de la vérité, montrent en même temps. que l'introduction des réserves dans les taillis est bien loin de racheter le désavantage que j'ai essayé de mettre en évidence, lorsque j'ai comparé ce mode d'exploitation à celui de la futaie : car, dans une futaie bien conduite, la part du produit, afférente au bois d'œuvre, peut s'élever aux trois quarts et quelquefois aux quatre cinquièmes.

\section{ARTICLE II.}

DE L'EXPLOITABILITÉ DANS LES TAILLIS SIMPLES.

La recherche de l'exploitabilité dans les taillis simples ne présente pas d'insurmontables difficul- 
tés, bien qu'il n'existe point de tables d'accroissement pour la faciliter. Il est.presque toujours possible de trouver, à proximité de la forêt que l'on aménage et dans des conditions semblables de végétation, des massifs suffisamment complets de bois d'âges divers, et de se procurer, par des places d'essai, les éléments d'appréciation nécessaires, pour déterminer l'âge d'exploitabilité, suivant la nature des produits que l'on veut se procurer.

En remplissant le tableau ci-contre, on aura sous les yeux les diverses solutions dont le problème est susceptible. 
DE L'EXPLOITABILITÉ.

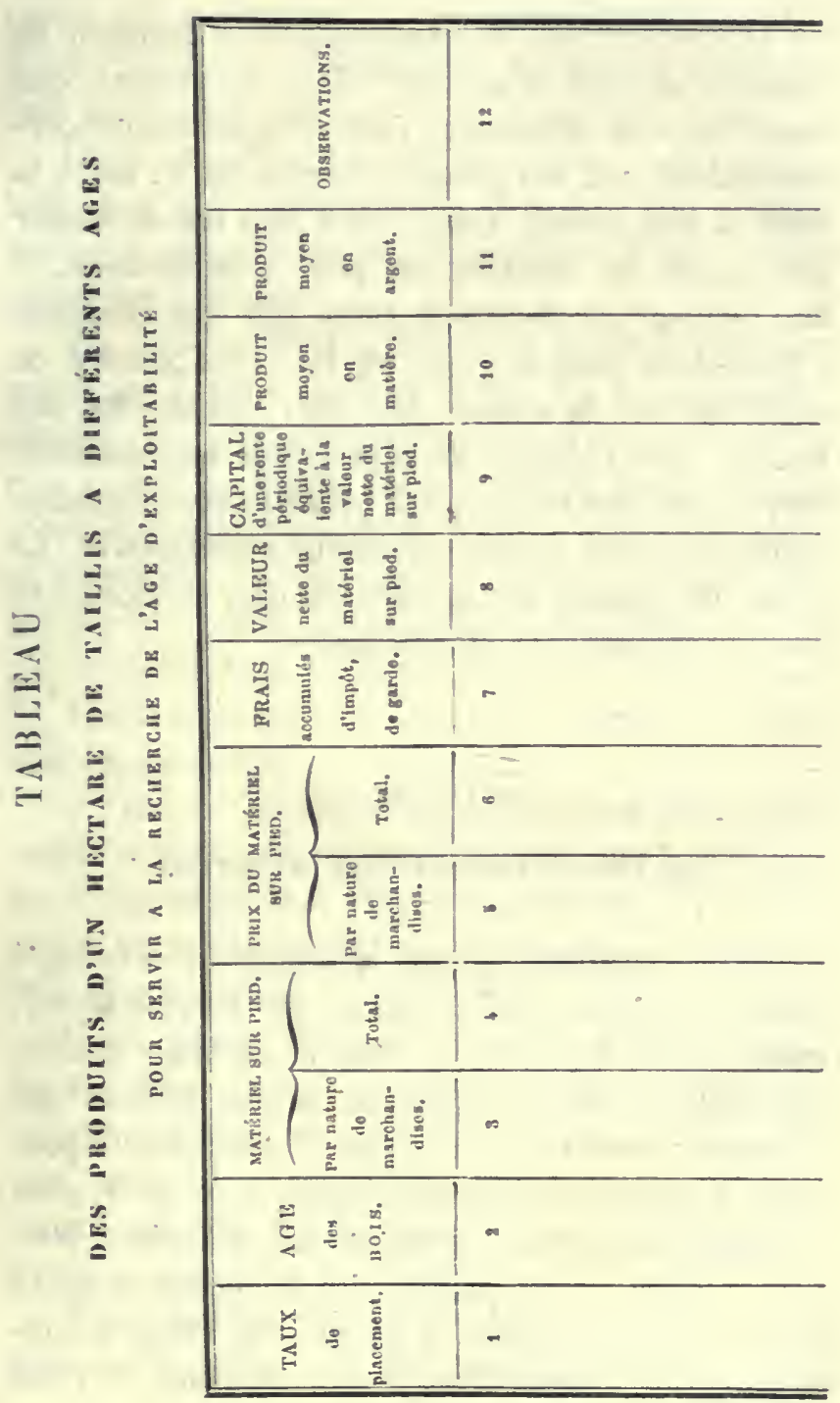


Les recherches, les expériences que comporte ce tableau, ne sont d'ailleurs utiles, en général, que lorsqu'on veut déterminer l'âge d'exploitabilité colrespondant, soit aux produits les plus utiles, soit à la rente la plus élevée. Quanc' on a pour but de retirer d'un taillis les produits les plus considérables, il faut pousser sa révolution aussi loin que possible, c'est-à-dire jusqu'à l'âge où les bois cessent de repousser par la souche. En effet, l'expérience démontre, que l'époque du plus grand accroissement moyen pour des rejets, ne se présente que longtemps après qu'ils ont perdu leur faculté reproductive. Le terme de l'exploitabilité dans ce cas, est donc fixé par une circonstance physiologique.

\section{ARTICLE III.}

DE L'EXPLOITABILITÉ DANS LES FUTAIȨS,

Nous avons déjà vu que les exigences de la régénération renfermaient le choix de l'exploitabilité, quelle qu'elle fût d'ailleurs, dans de certaines limites. En deçà de l'âge auquel les arbres portent des semences fertiles, il $n^{\prime} y$ a pas d'exploitabilité possible, à moins qu'on ne soit disposé à recourir, pour la régénération, aux repeuplements artificiels. Mais depuis l'âge où ils portent des semences jusqu'au terme de leur existence, il y a un long temps à courir ; et pour trouver dans ce laps de temps, le point 
précis qui correspond à une exploitabilité donnée, il faudrait des expériences qui malheureusement ne sont guère possibles dans l'état actuel des choses.

L'exploitabilité se détermine donc, dans les futaies, par des considérations plus ou moins vagues basées principalement sur la tradition et sur la longévité des essences.

En thèse générale, toutes les fois que l'on veut appliquer l'exploitabilité absolue, ou l'exploilabilité relative aux produits les plus utiles et au revenu le plus grand, on pousse les révolutions aussi loin que possible eu égard à la longévité des espèces. Lorsque, au contraire, on veut se procurer la rente la plus élevée, on avance le terme de ces révolutions autant que le comportent les exigences de la régénération.

Les raisons qui justifient cette manière d'opérer sont les suivantes:

$4^{\circ}$ Toutes les expériences faites sur des arbres isolés, et elles sont nombreuses, prouvent que le plus grand accroissement moyen de la plupart de ces arbres ne se réalise qu'à un âge très-avancé, lorsque déjà ils commencent à être altérés dans le cœur, et qu'ils ont perdu une partie de leurs qualités industrielles. Pour ce qui concerne ces arbres envisagés individuellement, on peut donc regarder comme certain, qu'en les coupant au moment où ils entrent en retour, on gagne plus pour la qualité et la valeur qu'on ne perd pour le volume.

Il est vrai que, ainsi que je l'ai déjà fait remarquer, ce n'est pas par des expériences individuelles 
que l'on doit essayer de déterminer les phases de l'accroissement d'un massif, mais par des expériences qui embrassent des massifs tout entiers. En opérant ainsi, ce qui, nous l'avons vu, est difficile, on trouve que l'époque du plus grand accroissement se présente avant celle du dépérissement. Ce fait, qui pourrait paraître au premier abord anormal, et qui est dû à la quantité, souvent considérable, de bois que les nettoiements et les éclaircies enlèvent à l'accroissement, ne saurait être admis sans explication.

Les nettoiements et les éclaircies ont pour but principal de favoriser l'accroissement; on peut dès lors supposer que si, au moment où on les pratique, ces opérations enlèvent des éléments à l'accroissement futur, elles rendent d'autant plus puissants ceux qu'elles respectent; et que, en conséquence, si on les opérait chaque année, chaque mois, chaque jour, le volume de bois qu'elles emporteraient, serait moindre que celui dont elles provoqueraient l'accroissement. Comment se fait-il donc que ces opérations aient cependant pour effet, non-seulement de modifier le rapport entre l'accroissement futur et l'accroissement passé, mais encore d'avancer l'époqne du plus grand accroissement moyen? - C'est parce que lorsque les massifs sont arrivés à un âge avancé, on ne se borne pas à en extraire tous les sujets nuisibles à leur développement; on enlève aussi ceux qui pourraient entraver l'amélioration de la qualité du bois; en sorte qu'il arrive un moment où la quantité de bois tombant dans l'éclaircie est plus grande 
que celle qu'on peut espérer de l'accroissement ultérieur. On comprend fort bien alors que les éclaircies aient pour résultat de hâter le termé de l'exploitabilité absolue. Dans tous les cas, cette influence sur le rapprochement de l'époque du plus grand accroissement moyen, ne saurait modifier sensiblement la loi naturelle qui veut que l'apogée de l'accroissement ne soit atleint généralement, que lorsque les bois ont déjà éprouvé un commencement de dépérissement. C'est donc, en définitive, l'état de santé des arbres qu'il faut surtout consulter, et d'après lequel on doit se guider, quand il s'agit de fixer. l'exploitabilité d'une forêt dont on veut retirer les produits les plus considérables; et il en est de même, presque toujours, lorsqu'on recherche les produits les plus utiles; car, sauf des exceptions peu nombreuses, l'utilité, on se le rappelle, est proportionnelle aux dimensions.

La détermination de l'exploitabilité relative soit aux produits les plus considérables, soit aux produits les plus utiles, dans les futaies comme dans les taillis, repose, on le voit, sur la constatation d'un fait matériel, constatation toujours possible, quoique cependant elle ne soit pas susceptible d'une précision parfaite.

- Quant à l'exploitabilité relative à la rente la plus élevée, le raisonnement a montré que l'accroissement des capitaux pécuniaires suit une marche progressive telle, qu'il y a presque toujours avantage à raccourcir autant que possible la révolution des massifs 
dont on veut retirer la plus forte rente. Dans la plupart des cas, l'époque de cette exploitabilité correspondra donc à l'âge auquel les bois sont susceptibles de se régénérer naturellement par les semences. Néanmoins, il sera utile, toutes les fois qu'un doute pourra s'élever à ce sujet par suite de la valeur extraordinaire des bois de certaines dimensions, de faire des expériences afin de s'assurer que cette valeur ne demande pas qu'on fixe l'exploitabilité à un âge plus avancé; et ces expériences sont suffisamment indiquées par le tableau ci-contre qui, bien exactement rempli, renfermera tous les éléments nécessaires pour se fixer sur l'âge correspondant à une quelconque des exploitabilités applicables à un massif. 
DE L'EXPLOITABILITÉ.

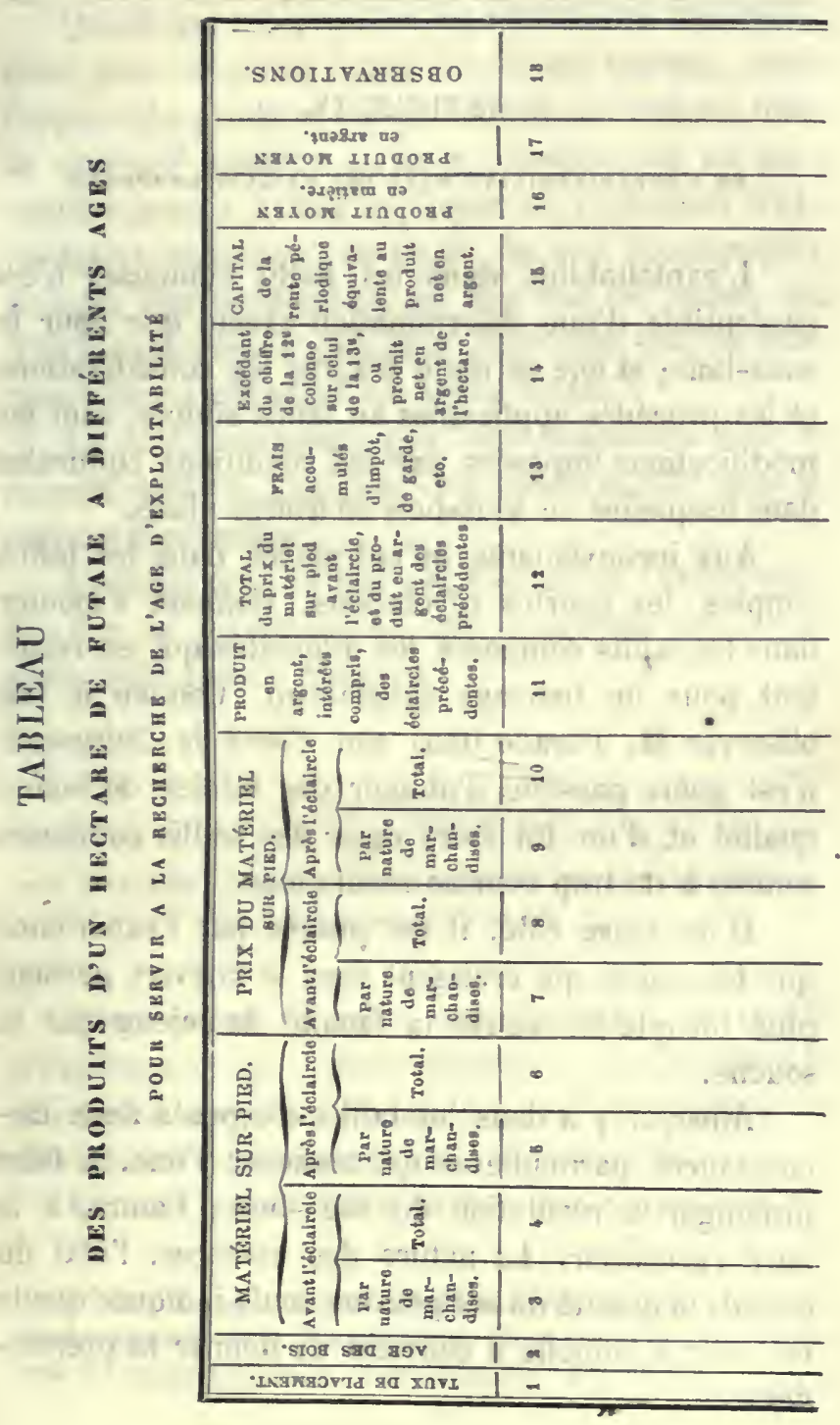




\section{ARTIGLE IV.}

DE L'EXPLOITABILITÉ DANS LES TAILLIS COMPOSÉS.

L'exploitabilité dans les taillis composés n'est susceptible d'une détermination exacte que pour le sous-bois; et elle se règle d'après les considérations et les procédés applicables au taillis simple, sauf les modifications imposées par leš conditions culturales dans lesquelles ce sous-bois se trouve placé.

Aux inconvénients qu'entraînent dans les taillis simples les courtes révolutions, viennent s'ajouter dans les taillis composés les difficultés qui en résultent pour un balivage satisfaisant. Comme le fait observer M. Parade dans son Cours de Culture, il n'est guère possible d'obtenir des futaies de bonne qualité et d'un fût élevé dans des taillis composés soumis à de trop courtes révolutions.

D'un autre côté, il est prouvé par l'expérience que les sujets qui croissent sous le couvert perdent plus tôt que les autres la faculté de rejeter par la souche.

Ainsi, il y a dans les taillis composés deux circonstances particulières qui tendent: l'une, à faire prolonger la révolution du sous-bois; l'autre, à la faire raccourcir. La nature des essences, l'état du climat, la qualité du sol peuvent seuls indiquer quelle - est celle à laquelle il convient de donner la prépondérance. 
Quant aux réserves, on en multipliera naturellement plus ou moins le nombre et les classes, selon l'espèce de produits qu'on voudra en retirer; mais de quelque manière qu'on's'y prenne, on ne parviendra jamais à leur appliquer un règlement d'exploitation rationnel, au point de vue économique; puisque leur destination, en tout état de cause, est - d'ètre exploitées à des âges différents qui ne sauraient être avantageux au même degré ${ }^{1}$ :

Le taillis composé, peu recommandable sous le rapport cultural, l'est peut-être moins encore sous le rapport économique.

Je terminerai ce que j'ai à dire sur l'exploitabilité par la récapitulation des desiderala que comporte cette grande question.

\section{ARTICLE, V.}

DES MESTRES a PRENDRE POUR ASSURER LA DÉTERMINATION DB L'EXPLOITABILITÉ.

Nous avons reconnu que l'absence de tables d'expériences sur l'accroissement et les propriétés physiques des bois rendait presque impossible la détermination 'rigoureuse de l'exploitabilité.

En effet, dans l'état actuel des choses, on est

1. Consulter, sur l'exploitabilité des taillis composés, le Cours d'aménagement de M. Nanquette, p. 6 a et suivantes. On y trouvera d'excellents conseils. (Note de la deuxième édition.) 
obligé de recourir, pour se faire une opinion sur le rendement futur dans un temps donné : soit à des expériences individuelles qui sont sans signification pour l'exploitabilité absolue, qui ne peuvent servir tout au "plus que pour l'exploitabilité - relative aux produits les plus utiles; soit à des cubages de massifs choisis dans la forêt même que l'on aménage, et s'éloignant plus ou moins de l'état régulier ou normal, végétant en tout cas dans des conditions différentes. Toutes ces expériences ne peuvent fournir que des résultats très-douteux. Il faudrait donc commencer en France, et les commissions d'aménagement seraient pour cela d'un grand secours, des expériences sur l'accroissement et l'utilité des bois, d'après une méthode bien définie. On ne saurait assurément avoir la prétention de constater les phases de l'accroissement et les qualités de chaque essence, suivant les mille conditions particulières, exceptionnelles, dans lesquelles elle pourrait se trouver. Parmi ces conditions, on devrait ne choisir que les plus tranchées, les plus caractérisées, eu égard au climat, à l'exposition, au sol. Je place le sol en dernière ligne, parce qu'il est établi que les soins ultérieurs et l'amendement qui en résulte, tendent à effacer les différences de qualité et de fertilité que semblerait impliquer la diversité de ses éléments constituants et primordiaux. Quels que soient au contraire ces soins, on n'arrivera jamais à obtenir dans un climat sec et froid une production aussi rapide que dans un climat chaud et humide. On ne fera jamais que, sous le même climat, 
la croissance ne soit pas plus rapide à l'exposition du nord parce que l'humidité y abonde, qu'à celle du midi parce que la chaleur y est trop intense par rapport à l'humidité.

Si l'on avait en France, suivant la latitude, l'altitude, l'exposition, la nature géologique du sol, pour chaque essence principale, des résultats d'expériences, indiquant, par périodes de 20 et même de 10 ans, l'accroissement possible de cette essence en quantité et en qualité, on pourrait fonder les plans d'exploitation sur des bases à peu près certaines et rationnelles. Jusque-là on ne fera guère que de l'empirisme. 


\section{GHAPITRE TROISIEME.}

DE L'EXPLOITABILITÉ DANS SES RAPPORTS AVEC L'INTÉRÊT DU PROPRIÉTAIRE.

Il résulte de la discussion à laquelle je me suis livré dans le chapitre I ${ }^{\text {er }}$ de cette étude :

$1^{\circ}$ Que le produit annuel en argent d'une forêt, considéré en lui-même et abstraction faite du capital engagé, s'accroît avec la durée de la révolution ;

$2^{\circ}$ Que le rapport entre ce produit et la valeur commerciale de l'immeuble diminue au contraire avec cette durée;

$3^{\circ}$ Que le taux des placements pécuniaires est la cause principale de cette contradiction qui disparâ̂trait si l'intérêt de l'argent devenait nul.

Ces lois remarquables expliquent et justifient les différences que nous avons déjà constatées dans les principes d'après lesquels l'Etat, les communes et les particuliers exploitent les bois qui leur appartiennent.

Nous allons, du reste, en examiner de plus près l'influence. 
ARTICLE PREMIER.

DE L'ESPLOITABILITÉ DANS SES RAPPORTS

AVEC L'INTÉRÊT DE L'ÉTAT.

L'État est éternel; l'intérêt de l'avenir doit done être mis sur la même ligne que celui du présent, lorsqu'il s'agit de régler l'exploitation des forêts qui lui appartiennent; et, poui satisfaire à ce principe dont la justesse me paraît incontestable, il est nécessaire d'abord d'adopter un mode d'exploitation qui ne puisse pas compromettre la conservation du domaine forestier; or ce mode est, sauf de rares exceptions, celui de la futaie.

Tous les membres de la société ont un droit égal aux produits des forêts de l'État. Ces produits ne sauraient dès Iors être regardés comme un objet de spéculation. Lorsque l'État les vend, il ne poursuit pas le même but que les autres propriétaires de bois. Il se sert de la vente comme du moyen le plus convenable, pour distribuer aux citoyens qu'il représente, un revenu qui n'appartient pas plus à l'un qu'à l'autre; il ne prétend pas d'ailleurs retirer de cette opération ưn profit commercial. L'État et la société ne faisant qu'un, l'État propriétaire de bois, quelle qué soit la.manière dont il en fait jouir le public, les consacre en réalité dans tous les cas à son usage, à sa consommation; et, partant, ce qu'il doit chercher à retirer de ses forêts, ' c'est sur un point 
donné la plus grande quantité des produits annuels les plus utiles. Les longues révolutions sont donc celles qu'il lui convient d'adopter en général, puisqu'elles tendent 'à augmenter la quantité et l'utilité de la production ${ }^{1}$.

Les profits du sol forestier ne consistent pas seulement dans la valeur de la coupe annuelle; ils consistent aussi dans les ressources que le bois offre au travail national, dans le courant commercial el industriel auquel il'donne lieu; ils consistent en outre dans l'influence heureuse que les forêts exercent par leur existence même sur les conditions climatologiques d'un pays.

Aucun de ces profits n'est perdu pour le propriétaire, quand ce propriétaire est l'ittat; et comme leur importance est en raison directe de l'utilité et de la quantité des produits et de la grosseur des arbres, il y a là encore de nouvelles raisons pour que l'État préfère les longues révolutions aux courtes.

L'avantage d'une prompte jouissance ne saurait compenser, pour l'État, la diminution de produits qu'entraîne nécessairement le raccourcissement des révolutions.

Il est certain que deux coupes de bois à

1. Ce n'est que très-exceptionnellement qu'il peut y avoir pour l'État intérét à en adopter de courtes; cependant, si une industrie indispensable â la șociété réclamait impérieusement des bois de petites dimensions et qu'on ne pût se les procurer que dans les forêts domaniales, il faudrait tenir compte de ces besoins dans le choix du traitement desdites forêts. 
l'âge de 50 aus donneront moinš qu'une seule coupe à l'âge de 100 ans; mais on objectera que, si on emploie le prix de la première coupe à 50 ans, d'une manière reproductive, jusqu'à l'époque de la seconde coupe au même âge, le profit qu'on en retirera pourra égaliser, s'il ne le dépasse, l'excédant du produit de la coupe à 100 ans sur celui des deux coupes à 50 ans. Cela est vrai, mais je ferai remarquer que cet excédant aura été donné en quelque sorte gratuitement par la nature, tandis que le profit qu'on lui compare, résultat des efforts de l'homme, aura exigé, consommé une main-d'œuvre qui aurait pu être utilisée autrement.

Pour que l'avantage d'une jouissance anticipée fût réel pour l'État, il faudrait deux choses : $1^{\circ}$ qu'il y eût dans notre pays surabondance de bois; $2^{\circ}$ qu'il ne fût pas possible de se procurer autre part que dans nos forêts des instruments de travail. Or, il y a au contraire pénurie de bois; et il n'est pas nécessaire de couper les forêts avant leur maturité, pour se procurer des instruments de production. Une opération qui aurait pour résultat d'amoindrir la puissance productive d'un capital, dont la nature a fait tous les frais, ne saurait donc trouver une justification suffisante dans l'importance du capital artificiel qu'on pourrait créer au moyen de cette diminution.

Il n'en est pas moins vrai cependant qu'au delà d'un certain âge l'accroissement d'un massif boisé ne représente plus, en valeur pécuniaire, l'intérêt de la somme qu'on réaliserait en le coupant; et on peut 
se demander si cet intérêt doit être exclu des considérations qui doivent présider à la gestion des forêts de l'Etat.

Il y a une raison péremptoire pour que cette question soit résolue par l'affirmative :

Cette raison, c'est que l'intérêt de l'argent n'implique pas une augmentation de revenu; c'est qu'en supposant un moment qu'il pût être supprimé, sans que la circulation des capitaux en souffrît, la quantité d'utilités créées, de valeurs produites au bout de l'année, n'en serait pas altérée. Il résulte de là que. l'intérêt constitue un bénéfice pour les individus ou pour une fracion de la société considérée par rapport à la masse des citoyens, parce qu'il modifie, en faveur de ces individus ou de cette fraction de la société, la répartition du revenu social; mais qu'il ne saurait, dans aucun cas, constituer un bénéfice pour l'État.

Cette vérité a longtemps été méconnue; aussi voyons-nous encore une grande partie des forêts de l'État exploitées en taillis; mais les saines doctrines paraissent avoir enfin définitivement prévalu. L'administration est entrée dans la voie des conversions en futaies; on ne saurait trop l'en fẻliciter. 


\section{ARTICLE II.}

DE L'EXPLOITABILITÉ DANS SES RAPPORTS AVEC L'INTÉRÊT DES PARTICULIERS.

Les besoins impérieux de la vie, l'imprévoyance, les partages amenés par nos lois sur les successions, sont autant de motifs qui s'opposent à ce que les bois appartenantaux particuliers soient exploités à un âge reculé.

L'État a toujours lés moyens de suppléer, par d'autres ressources, à celles que lui fournirait la coupe de ses bois. Les particuliers les ont rarement, ils peuvent être et ils sont souvent forcés de sacrifier toutes les espérances de l'avenir aux nécessités du présent.

D'un autre côté, quel que soit l'esprit de prévoyance dont on suppose les hommes doués, il est concevable qu'il s'altère et s'affaiblisse, lorsqu'il embrasse un laps de temps qui dépasse la durée de leur existence.

Enfin, cet esprit, il faut le reconnaître, trouve encore une cause de découragement dans la certitude que la propriété qu'il sera parvenu à constituer, au prix des plus grandes privations, sera tôt ou tard démembrée, par suite des lois qui régissent, en France, l'hérédité.

On' s'explique donc que les particuliers ne soient guère tentés de conserver longtemps leurs bois sur pied; mais la raison qui les en éloigne surtout, est le 
désir bien naturel d'augmenter, autant que possible, le rapport entre le revenu net et la valeur vénale de cette nature de biens-fonds.

Si le taux de placement adopté pour les bois est de 3 pour 100 , les particuliers propriétaires de bois tendront à les exploiter à l'âge au delà duquel l'augmentation de valeur qui résulterait de l'accroissement annuel cesserait d'être égale à l'intérêt, à 3 pour 100 , que fournirait le produit pécuniaire de l'exploitation joint à la valeur de la première feuille. D'après les prix qu'ont actuellement les bois suivant leur grosseur et leur âge, il ne faut pas laisser longtemps sur pied une forêt pour qu'elle cesse de rapporter, à 3 pour 100 , l'intérêt de sa valeur. Voilà pourquoi nous voyons les particuliers exploiter en général celles qu'ils possèdent, en taillis simples et à l'âge de 20 ans au plus, toutes les fois que les essences le permettent.

Cette manière d'agir est rationnelle : elle est théoriquement justifiée par les principes que nous avons développés dans le premier chapitre de la présente étude. Toutefois elle ne saurait être recommandée sans réserve, et elle ne convient même véritablement qu'aux propriétaires qui ont besoin de vendre leurs bois pour satisfaire aux exigences de leur consommation. Quant à ceux qui cherchent à spéculer sur le produit de leurs coupes, ils seront amenés par l'intelligence pratique de leurs intérêts, à maintenir souvent leurs bois sur pied au delà du terme fixé par la théorie. 
En effet, ne perdons pas de vue que dans nos calculs sur l'exploitabilité relative au taux de placement, nous avons tenu compte des inlérêts des intérêts, et qu'en conséquence, pour qu'un propriétaire trouvât son profit à appliquer rigoureusement les principes que nous avons posés, il faudrait qu'il eût toujours la possibilité de faire croître, à intérêts composés, le produit pécuniaire que lui procurerait l'exploitation de son bois.

L'accumulation des capitaux pécuniaires à intérêts composés est-elle facilement réalisable? - Je ne le crois pas, et il me semble même que les emprunteurs seraient tout à fait impuissants à satisfaire les capitalistes, si ceux-ci prétendaient tous faire leurs placements à intérêts composés. En supposant que les premiers se résignassent à se priver de tout profit net, et à ne prélever sur le produit des capitaux empruntés que ce qui serait strictement nécessaire à leur existence, l'excédant ne suffirait certainement pas au service des intérêts composés. Pour s'en convaincre, l'on n'a qu'à consulter les statistiques les plus estimées sur l'augmentation de la richesse publique, dans les sociétés qui disposent des moyens de production, même les plus puissants. On y verra que la proportion dans laquelle cette augmentation a eu lieu, est fort éloignée de celle que comporterait la puissance de l'accumulation des capitaux à intérêts composés. Je ne sache pas, au reste, qu'il y ait beaucoup dé banquiers disposés à servir l'intérêt des intérêts des sommes déposées chez eux, et que les 
établissements qui en avaient pris l'engagement aient jamais pu le tenir.

Doit-on conćlure de là que des placements à intérêts composés ne sont, dans aucun cas, réalisables? - Assurément non; seulement, on doit les considérer comme tout à fait exceptionnels ei sortant des conditions normales de la production; on doit les considérer comme d'autant plus difficiles que leur durée devra être plus longue.

Un propriétaire qui n'aura pas besoin de couper son bois, pour subvenir à des dépenses urgentes, agira donc sagement en le laissant sur pied au delà du terme où l'accroissement ne représentera plus le profit qu'il pourrait exceptionnellement retirer de la coupe, s'il en plaçait le prix. Son bois continuera de s'accroître à intérêts composés, à un taux moins élevé, sans doute, que celui qu'on recherche et qu'on peut obtenir dans les placements de cette nature; mais dans des conditions de sécurité que ne présentent pas les placements pécuniaires.

$\mathrm{Au}$ surplus, quand on veut retirer des bois une rente plus élevée que celle qu'on retire des terres arables, on a, selon moi, une prétention qui n'est pas raisonnable.

Les bois, il est vrai, ne sont pas susceptibles d'être affermés; ils sont difficiles à estimer; le prix de leurs produits est sujet à de grandes fluctuations; ils ne jouissent pas, d'autant de crédit que les.autres capitaux ou fonds de terre, par suite de la facilité avec laquelle on peut enlever, par l'exploitation de 
la superficie, la garantie que le prêtenr cst en droit de réclamer. Toutes ces circonstances tendent à les déprécier et à augmenter, par conséquent, la rente qu'on cherche à en retirer; mais les bois rachètent les inconvénients que je viens d'énumérer par un précieux privilége; et ce privilége consiste précisément en ce que leur exploitation peut être retardée. lorsque les circonstances ne sont pas favorables à l'ćcoulement de leurs produits.

Si vous avez de l'argent chez un banquier ou ('hez tout autre, et qu'à l'époque où vous avez à en loucher l'intérêt, vous ne sachiez que faire de cet intérêt, il faudra le garder improductil dans votse secrétaire. Si vous possédez un chainp de blé, et qu'à l'époque de la récolte, l'état du marché ne vous pcrmette pas de vendre cette récolte à un prix rémunérateur, vous essayerez de la conserver dans des greniers, ou dans des silos construits à grands frais.

Pour le bois, c'est la nature elle-même qui se charge de la conservation des produits, et qui, pour cela, ne vous demande qu'une légère diminution sur l'intérêt qu'elle vous avait bonifié jusqu'alors. Les circonstances sont-elles contraires à la vente de la récolte, on laisse celte récoltẹ sur pied. Non-seulement elle s'y conserve, mais elle s'y améliore; et tandis qu'on s'expose pour le blé, par exemple, à en trouver, au bout d'un certain temps, une grande partie avariée, mangée par les rats, rongée par l'alucite, on est certain, au contraire, de retrouver le 
bois dont on aura retardé la coupe, amélioré par les années qui se seront accumulées sur lui.

Les bois sont donc de toutes les caisses d'épargne, la plus commode; la plus sûre, la plus fidèle, et c'est vraiment trop exiger d'eux, que de leur demander une rente plus élevée que celle qu'on retire des autres biens-fonds.

ARTICLE III.

DE L'EXPLOITABILITÉ DANS SES RAPPORTS AVEC L'INTÉRET DES COMMUNS.

L'existence des communes ne peut pas plus ètre limitée que celle de l'État. Dans la gestion de leurs biens patrimoniaux, les communes doivent donc, avant toute chose, se préoccuper des moyens d'assurer la conservation du fonds; et dans ce but, c'est le mode d'exploitation en futaie qu'il leur convient en général d'adopter pour les forêts qu'elles possèdent.

Mais les communes diffèrent de l'État et se rapprochent des particuliers par la modicité de leurs ressources d'abord, et ensuite par la distinction qui existe entre les intérêts de chacune d'elles et les intérêts généraux du pays. Lorsque, par le placement de leurs capitaux, elles peuvent faire modifier en leur faveur la répartition du revenu social, n'ontelles pas raison de recourir à ce moyen pour améliorer leur sort? 
Je répondrais négativement à cette question, si toutes les communes étaient dans l'aisance, si leur revenu était suffisant pour couvrir leurs dépenses; parce que, comme je l'ai fait observer dans une étude sur les quarts en réserve des bois communáux ${ }^{1}$, la conservation d'un capital - sous la forme d'une forêt, présente à côté de l'inconvénient de faire produire à ce capital une rente inférieure à celle qu'on en retirerait en lui donnant une autre destination, en le itransformant par exemple en argent, l'avantage d'une sécurité beaucoup plus grande. Cet avantage est inappréciable pour les propriétaires qui ne meurent pas. Cependant, de même que le particulierqui, n'ayant pas de quoi suffire aux strictes nécessités de la vie avec le revenu d'une terre, fait bien de la vendre et d'en placer la valeur chez un banquier, malgré les chances de ruine auxquelles il s'expose; de même une commune qui a des besoins impérieux et des recettes trop restreintes, est excusable de chercher à remédier à cette situation, en renonçant à conserver des propriétés productives d'un trop faible revenu relativement à leur valeur commerciale.

Toutefois, il ne faut pas qu'elles oublient que ce genre de spéculation, qui a pour résultat de raccourcir la durée de la révolution de leurs bois, est contraire à l'intérêt de leurs descendants dont il ébranle le patrimoine, et à l'intérêt de la société

1. Annales forestières, 1856. 
dont il amoindrit le revenu. Si les particuliers eux-mèmes ne doivent jamais faire complétement abstraction de ces intérêts, leurs obligations à elles sont, sous ce rapport, beaucoup plus étendues.

L'importance de ces obligations est suffisamıment indiquée par la non-limitation de l'existence des communes, par l'étroite solidarité qui existe entre elles et la société dont elles font partie, par la protection spéciale dont l'État les couvire, par l'appui qu'il leur prête. 


\title{
CHAPITRE QUATRIÈME
}

\author{
DE L'EXPLOITABILITÉ CANS SES RAPPORTS \\ AVEC L'IXTÉRÈT PÜBLC
}

Je voudrais maintenant calculer la perte que fait éprouver à la France l'exploitation en taillis d'une partie des bois qui sont situés sur son territoire. Cette perte est énorme: A défaut d'expériences assez concluantes, je ne saurais la préciser; mais je crois qu'il me sera facile d'en donner une idée trèsapproximative.

Or lit dans le Cours de culture, page 399, que Hartig, ayant comparé entre eux (toutes circonstances égales d'ailleurs) un taillis simple exploité à 30 ans et une futaie soumise à une révolution de 120, a trouvé que les produits en matière de ces deux forêts étaient dans le rapport de 4 à 7 .

J'appliquerai ce rapport aux taillis simples et aux futaies en général.

Je supposerai en outre que, par suite de la présence des réserves dans les taillis, les produits du taillis composé soient à ceux de la futaie comme 5 est 7.

J'admettrai enfin qu'une futaie, dans des condi- 
tions moyennes de végétation, et bien traitée, puisse donner par hectare 6 mètres cubes, chiffre très-modéré.

Le taillis simple en fournira dès lors 3,45

Et le taillis composé. . . . . 4,30

Or, en France; le sol forestier comprend en chiffres ronds, $8,500,000$ hectares $^{1}$.

S'il était couvert de futaies convenablement cultivées, il pourrait donc fournir annuellement 51 millions de mètres cubes.

Yoyons ce qu'il serait possible d'en retirer, en conservant les divers modes d'exploitation qui le régissent actuellement.

Les $8,500,000$ hectares de forêts qui existent en France compremnent à peu près, 2,300,000 hectares de futaies. A raison de 6 m.c. p. h., on pourrait en retirer chaque année . . . 13,800,000 m. c.

On ne sait pas quelle est la part afférente au taillis composé ou au taillis simple, dans les

$$
1 \text { reporter. . . 13,800,000 m.c. }
$$

1. Ces chiffres ne sont plus tout à fait exacts, puisque la Prusse nous a pris environ 350,000 hectares de forêts domaniales et communales; mais quand j'ai publié ces études, nous n'avions pas la Savoie et le comté de Nice, qui nous ont apporté à peu près $\mathbf{6 0 , 0 0 0}$ hectares de forêts de même nature, et l'étendue que j'attribuais aux bois des particuliers était un peu faible; 'de sorteque la différence entre l'étendue totale du sol forestier qui existait lors de la $4^{\text {re }}$ édition de ce livre et celle qui existe aujour-. d'hui, ne saurait modifier sensiblement les conséquences que je tire de mes calculs. (Note de la qe édition. 
Report . ... 13,800,000 m. c.

$6,200,000$ hect. qui ne sont pás cultivés en futaie. En attribuant une part égale à chaque nature de peuplement, on exagérera plutôt qu'on ne diminuera la production; car la plus grande partie des bois en question appartient à des particuliers qui, on le sait, ont un goût prononcé pour le taillis simple.

$3,100,000$ hect. de taillis composés fourniraient, chaque année, à raison de $4 \mathrm{~m}$. c. 30 p. h. ....... 13,330,000

$3,100,000$ hectares de taillis simples fourniraient, chaque année, à raison de 3 m. c. 45 p. h. $\quad 10,695,000$

$$
\text { Total. .... }
$$

Ainsi, le sol forestier, couvert de futaies, pourrait rapporter chaque année, 51 millions de m.c.; tandis que le maximum de la production possible, avec les diverses méthodes d'exploitation auxquelles il est actuellement soumis, ne saurait dépasser 38 millions de m. c. La différence entre ces deux chiffres, soit 13 millions de m. c.; exprime la perte matérielle qui résulte pour la société de la préférence donnée au taillis sur la futaie.

Cette perte deviendra bien plus remarquable, si 
nous recherchons la qualité des produits qu'elle représente.

On sait que dans certaines futaies le bois d'œuvre absorbe jusqu'à 80 pour 100 du produit total; nous en porterons la quantité à 50 pour 100 seulement.

Dans un taillis composé normal, la futaie n'entre pas pour plus de un tiers dans le produit de la coupe annuelle; et en admettant que sur cette futaie la moitié donne du bois d'œuvre, nous resterons audessus de la vérité. Si donc je fixe à un sixième du produit la part afférente au bois d'œuvre dans les taillis composés, je ne serai pas accusé de vouloir l'atténuer. Dans les taillis simples, la part du bois d'œuvre est presque insignifiante. J'en tiendrai pourtant compte en la portant à un dixième.

D'après ces suppositions, le produit du sol forestier se composerait :

$1^{\circ}$ Dans l'hypothèse du traitement en fulaie,

De 51 millions de mètres cubes dont :

En bois d'œurre.

En bois de feu.

$25,500,000$ m. c. $25,500,000$ m. c.

$2^{\circ}$ Dans l'hypothèse du maintien des modes actuels d'exploitation,

De 13,800,000 mètres cubes fournis par les futaies et donnant:

En bois d'œurre.

En bois de feu.

$6,900,000$ m. c. $\quad 6,900,000$ m. c.

\section{De $13, \hat{\jmath} 30,000$}

mètres cubes

A reporter. . $6,900,000 \mathrm{~m}$. c. $6,900,000 \mathrm{~m}$. c. 
Reports . . 6,900,000 m. c. $\quad 6,900,000$ m. c. fournis parles

taillis compo-

sés el don-

nant. . . 2, 225,000

$11,105,000$

De $10,695,000$

mètres cubes

fournis par les

taillis simples

et donnant . $\frac{1,069,500}{10,194,500 \text { m. e. }} \frac{9,625,500}{27,630,500^{\text {m.e. } 1} \text {. }}$

Si nous retranchons la quantité de bois d'œuvre produite dans la seconde hypothèse, de celle produite dans la première, nous trouvons une différence de 15 millions 400 mille mètres cubes en chiffres ronds.

Si nous retranchions la quantité de bois de feu produite dans le premier cas, de celle produite dans le second, nous trouvons une différence de 2 millions 130 mille mètres cubes en chiffres ronds.

Et si nous évaluons ces différences en argent, à raison, pour le bois d'œuvre, de 20 francs sur pied, prix très-modéré, et, pour le bois de feu, de $7 \mathrm{fr}$., prix assez élevé, nous constaterons, en définitive, que l'exploitation en taillis fait perdre à la sociéte un revenu de :

1 Je n'ai pas besoin de rappeler que ces produits dépassent beaucoup les produits réels, et sont ceux que les forèts pourraient donner, si elles étaient entretenues corivenablement. 
$15,400,000 \mathrm{m.c} \cdot \times 20 \mathrm{fr} .=308,000,000 \mathrm{fr}$.

$-\quad 2,130,000^{\mathrm{m} . c \cdot} \times 7 \mathrm{fr} .=14,910,000 \mathrm{fr}$.

Différence. . . 293,090,000 fr.

293 millions de francs! C'est plus de la moitié de nos contributions directes, c'est plus que l'intérêt de notre contribution de guerre.

Mais il faul, pour embrasser dans toute son étendue le préjudice qu'est susceptible de causer au corps social le mode d'exploitation en taillis, réfléchir à la quantité énorme de travail, que les $\mathbf{1 5}$ millions de mètres cubes de bois d'œuvre dont on pourrait augmenter la production forestière, en adoptant le mode de la futaie', créeraient dans toutes les branches de l'industrie.

J'ai montré dans une note sur le défrichement. que les bois formaient en France le tiers à peu près, en poids, des matières transportées.

D'un autre côté, on lit dans le procès-verbal de l'enquête qui a été faile en 1847 sur l'industrie parisienne, que la valeur des produits fabriqués, pour les industries n'employant, comme matière première que le bois, s'élevait à 101,516,026 francs; que classées par rang d'importance dans cet-immense atelier de la capitale, la charpenterie occupait le $20^{\mathrm{e}}$ rang, la carrossserie le $16^{\mathrm{e}}$, l'industrie du bâtiment le $9^{\text {e}}$, l'ébénisterie le $8^{\text {" }}$; que le nombre des patrons et ouvriers occupés à la manipulation du bois dépassait enfin 35,000 .

Ces renseignements me paraissent assurément 
propres à dissiper toute incertitude sur l'immense intérêt qu'a la société, à ce que l'on adopte pour les forêts dont elle jouit, des exploitabilités reculées.

II n'est pas permis d'espérer que les particuliers trouveront jamais leur intérêt à exploiter leurs bois en futaie; car, pour cela, il faudrait non-seulement que le loyer de l'argent baissât considérablement; mais, ce qui est plus difficile à réaliser, que l'imprévoyance, le besoin du moment, l'incertitude du lendemain, cessassent d'intervenir dans les actions humaines.

C'est donc à l'amélioration des forêts de l'État et des communes que l'on doit songer, et le lecteur sait maintenant dans quel sens il importe de la diriger.

On n'apprécie pas assez dans notre pays, la place que les forêts occupent dans le domaine où la société puise incessamment les matériaux, les éléments qui peuvent servir à son existence, à son bien-être, au développement de sa prospérité. Chose remarquable! le bois est indispensable à la plupart des industries : presque tous les meubles dont l'homme se sert, sont en bois, depuis le berceau qui l'a reçu à sa naissance, jusqu'au cercueil qui le contiendra après sa mort. C'est avec le bois qu’il prépare ses aliments, réchauffe ses membres engourdis par le froid, construit son habitation, laboure son champ, transporte ses denrées; c'est le bois qui lui a permis d'étendre son empire sur' l'Océan, et de créer ces voies de fer qui ne feront bientôt du monde entier qu'une seule famille. Supprimez le bois, toutes les fonctions sociales sont interrompues; tout s'arrête én même temps, les travaux 
de la paix comme. ceux de la guerre, les travaux qui donnent le nécessaire comme ceux qui donnent le luxe. Enfin, de quelque côté qu'on jette les regards, le bois se présente à nous comme l'auxiliaire le plus puissant que la Providence ait mis à notre disposition pour améliorer notre sort; et pourtant, de toutes les ressources qui nous environnent, c'est celle dont nous nous préoccupöns le moins. Aussi les forêts s'en vontelles avec une évidente et alarmante rapidité; la spéculation les poursuit à outrance, et si l'on n'y prend garde, elle en aura bientôt fait table rase ${ }^{1}$.

1. Quelques années à peine se sont écoulées depuis le rejet par le Corps-législatif du projet d'aliénation présenté sous l'empire par le ministre des Finances, et déjà l'on voil se reproduire des propositions dans le mème sens, propositions toujours appuyées sur les mèmes considérations, les mènes sophismes : I's besoins de l'agriculture, les ressources des bois de particuliers, la faible rente des forêts de l'Etat, l'existence dans certaines contrées de forêts indéfinies et inépuisables, etc.; etc.

On oublie que nous avons encore en France plusieurs millions d'hectares qui ne sont couverts que de bruyères; et que par conséquent le terrain ne manque pas à l'agriculture;

On oublie que les particuliers défrichent de plus en plus et qu'ils y sont poussés, d'un côté, par l'impossibilité pour eux d'éleverdes futaies, de l'autre, par la dépréciation du bois de chauffage;

On oublie que la faible rente des forêts de l'État est précisćment la preuve do l'intensité de leur culture el de la supériorité de leurs produits;

On ignore enfin qu'ıl n'y a de forêts indéfinies el inépuisables nulle part; qu'on défriche aujourd'hui avec une sorte de fureur dans toutes l's parties du monde, et que les Américains euxmêmes n'ont plus assez de bois pour leur propre consommation. (Lire à ce sujet un intéressant article de M. G. Fabre dans la Revue des eaux el forêls, fér. 1872.) 


\section{TROISIÈME ÉTUDE.}

DU PLAN D'EXPLOITATION.

\section{CHA PITRE PREMIER.}

PRINCIPES FONDANENTAUS.

Quand on a complété la statistique de la forèt à aménager; quand on a terminé la description spéciale des parcelles dont elle se compose; quand on a choisi le mode d'exploitation et fixé l'àge d'explo:tabilité qu'on estime être applicables à ces parcelles, on possède les éléments nécessaires pour arrèter la durée de la révolution, la quotité et la marche des coupes annuelles.

Les documents relatifs à ces divers objets forment ce qu'on appelle le plan d'exploitation.

J'ai dit que le mot Révolution s'appliquait au temps nécessaire pour qu'une série de coupes de régénération pùt revenir au point de départ et se renouveler - dans les mèmes conditions. C'est' là le vrai sens du mot et par conséquent, si on voulait 
s'exprimer correctement, on devrait n'employer ce mot que pour l'exploitation des forêts normales, car' c'est dans les forêts normales seulement que les coupes de régénération peuvent se renouveler périodiquement dans les mêmes conditions. Mais cependant, par suite de la pauvreté de notre langue, on a abusé du mot Révolution comme de beaucoup d'autres, et on l'a employé indifféremment pour les forêts qui sont susceptibles d'un aménagement définitif et pour celles qui ne le sont point, de sorte qu'il ne se lie pas toujours à l'idée d'une rotation régulière de coupes d'une nature déterminée.

Il en est ainsi, par exemple, lorsqu'on se sert du mot Rérolution pour exprimer le temps pendant lequel une forêt devra être parcourue par des coupes préparatoires à la futaie. Il est évident que ces coltpes préparatoires ne sont pas destinées à se renouveler indéfiniment; que souvent même, on n'attend pas qu'elles aient parcouru toute l'étendue de la forèt, pour entreprendre les coupes de régénération. Le mot Révolution ne leur convient donc pas grammaticalement. L'usage l'a pourtant admis et consacré et je ne prétends pas le proscrire. Seulement, pour prévenir toute confusion, je prendrai les précautions suivantes :

J'appellerai Révolution principale normale le temps nécessaire pour l'exploitation, pour la régénération totale d'une forêt, lorsque ce temps correspondra à l'exploitabilité des essences; Révolution principale transitoire celle qui bien que s'appliquant aux 
coupes de régénération, ne différera de la précédente que parce qu'elle devra exceptionnellement ètre plus courte ou plus longue que ne le comporterait l'exploitabilité des essences.

Mais puisqu'il est fort embarrassant de déterminer l'âge d'exploitabilité d'une forêt, mème lor'sque cette forêt est dans un état que l'on peut croire stable au point de vue de la consistance et de la végétation des peuplements qui la composent, le problème devient bien plus difficile à rẻsoudre quand elle est évidemment destinée, sous ce double rapport, à éprouver des changements; 'de sorte qu'à l'incertitude des circonstances économiques s'ajoute celle des circonstances physiques. Telle exploitabilité excellente aujourd'hui, susceptible par conséquent d'être considérée comme normale, pourra ne plus l'ètre. dans un siècle. Aussi sera-t-il raisonnable d'adopter comme normale ou définitive toute révolution principale qui n'imposera pas des sacrifices probablement trop grands d'accroissement et d'utilité.

J'appellerai Révolution préparatoire, bien que ce soient les coupes et non la révolution qui préparent, le temps pendant lequel une forêt devra être parcourue par.des coupes qui auront pour but d'y rendre possible le début des coupes de régénération. On s'estquelquefois servi, dans ces dernières années, pour exprimer ce temps, des mots période d'attente. Or la période avait toujours été prise jusqu'alors dans une acception restreinte. C'était et c'est encore une partie de la révolution qui ne saurait se dérouler en 
dehors d'elle; il y aurait des inconvénients à lui donner une autre signification.

La révolution préparatoire, telle que je la comprends, remplacera dans certains cas, la révolution transitoire telle qu'elle est définie par MM. Lorentz. et Parade.

L'établissement du plan d'exploitation repose sur plusieurs principes essentiels et qui consistent :

1. $\Lambda$ faire arriver autant que possible chaque parcelle en tour de régénération, à l'époque correspondante à son âge d'exploitabilité et, en tour d'exploitation intermédiaire, à l'époque indiquée par l'état du peuplement;

2. A prescrire pour les exploitations successives des parcelles, un ordre qui soit conforme aux règles sur l'assiette des coupes;

$3^{\circ}$ A former pour la répartition des produits, dans le cours de la révolution, un règlement qui, tout en se conciliant avec les exigences de la culture, soit de nature à assurer le rapport annuel soutenu.

L'application de chacun de ces principes'est soumise à des conditions particulières plus ou moins importantes, plus ou moins impérieuses, et souvent opposées.

Cette opposition est une des plus grandes difficultés que l'on rencoṇtre dans l'établissement du plan d'exploitation.

Cet établissement varie suivant les modes d'exploitation; nous l'étudierons d'abord, dans le cas le 
moins compliqué, c'est-à-dire en admettant que la forêt à aménager doive être exploitée en taillis simple; nous verrons ensuite quelles modifications il faut y apporter, quand il s'agit de former une forêt régulière, exploitée d'après la méthode du réensemencement naturel et des éclaircies périodiques.

Quant aux taillis composés, comme ils ne diffèrent des taillis simples que par l'importance de la réserve, et comme d'ailleurs cette importance est subordonnée à des circonstances culturales très-variables, je ne crois pas nécessaire de faire de leur aménagement l'objet d'une étude particulière ${ }^{1}$.

1. Le lecteur qui aurait besoin d'être mieux fisé sur la question de l'aménagement des taillis composés, consultera avec fruit le livre de $M$. Nanquette, pages 297 et suivantes. (Note de la 2e édition.) 


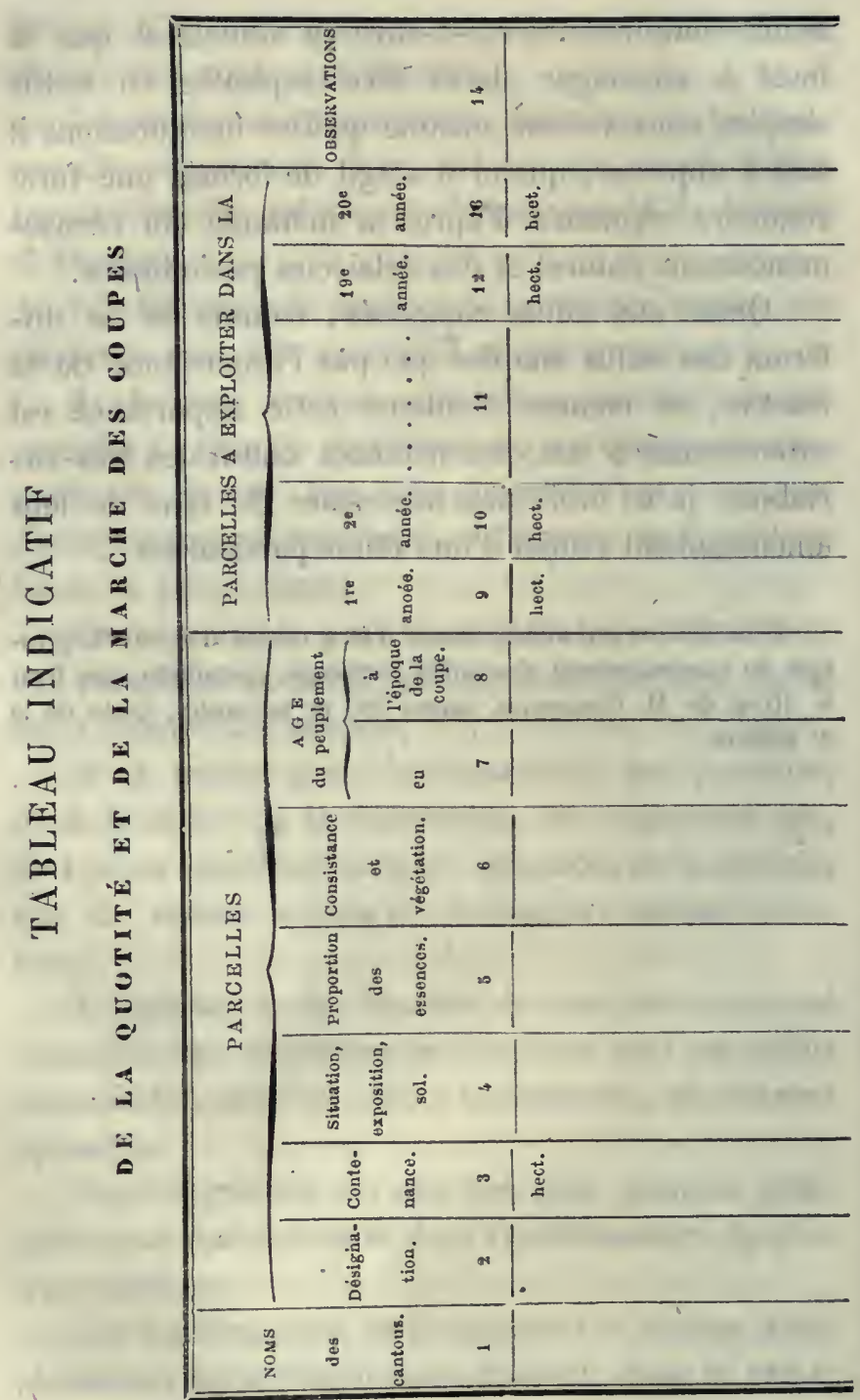




\section{CHAPITRE DEUXIÈME.}

DU PLAN D'EXPLOITATION DANS LES-TAILLIS SIMPLES.

\section{ARTICLE PREMIER.}

DU TABLEAU DES EXPLOITATIONS ET DES DIFFICULTÉS

QUE RENCONTRE SA FORYATION.

Nous avons affaire à un taillis dont la contenance est de 200 hectares, dont les parcelles sont toutes exploitables à 20 ans, et par conséquent dans une révolution principale de 20 ans ${ }^{1}$. Nous avons sous les yeux le cahier de la description spéciale et le plan topographique sur lequel les parcelles ont été figurées avec l'indication de leur étendue et de leur âge respectifs. Nous remplirons le tableau ci-contre :

Pour savoir quelle est celle des 20 dernières colonnes à laquelle appartient une parcelle quelconque, il suffira de prendre la différence entre l'âge d'exploitabilité et l'âge actuel de cette parcelle; si la différence était de 5 années, ce serait dans la cin-

1. Il est bien enteudu que ce chiffre de 20 ans est arbitraire. On n'en a pas pris un plus élevé, afin de simplifier la démonstralion. (Note de la z'édition.) 
quième colonne qu'il faudrait placer la parcelle en question; si elle était nulle ou négative, ce qui indiquerait que la parcelle est exploitable depuis plus ou moins longtemps, on la colloquerait dans la première colonne.

Au lieu du classement rigoureux que je suppose ici et qui implique que dans la reconnaissance de la forêt on est parvenu à distinguer les peuplements dont les âges ne diffèrent que d'un an ${ }^{1}$, on pourrait adopter un classement de 5 en 5 ans, et remplacer les 20 dernières colonnes du tableau par 4 colonnes seulement, dont la première comprendrait les bois exploitables dans la première période de $\mathbf{1}$ à 5 ans; la deuxième, les bois exploitables dans la deuxième période de 6 à 10 ans, etc., etc. Je conserverai néanmoins le classement que mes lecteurs ont sous les yeux, parce qu'il est propre à rendre plus intelligibles les observations auxquelles la formation d'un plan d'exploitation est susceptible de donner lieu.

La contenance de la forêt étant de 200 hectares, l'âge d'exploitabilité de 20 ans, il est clair que, si toutes les parcelles étaient placées dans les mêmes conditions de végétation et présentaient la gradation d'àgè convenable, on devrait trouver au total de chacune des 20 dernières colonnes du tableau un

1. Contrairement aux règles que j'ai exposées au sujet du parcellaire, pages 48 et suivantes, règles qui n'admettent pas non plus qu'il faille plusieurs parcelles pour former une coupe. (Note de la $2^{\mathrm{e}}$ édition.) 
chiffre égal au quotient de l'étendue de la forêt par l'àge d'exploitabilité, et, par conséquent, 10 hectares.

Si, d'un autre côté, en rapprochant l'assiette des coupes résultant de ce classement de celle que prescrivent les règles de la culture, on constatait qu'elle n'y déroge pas, on en conclurait que ledit classement ne laisse rien à désirer, et il n'y aurait plus qu'à l'appliquer sur le terrain, au moyen de laies sommières et séparatives, de fossés, de bornes et de numéros indicateurs.

Le plan d'exploitation se trouverait parfaitement en harmonie avec les principes que nous avons posés dans le premier chapitre de cette étude : la production maxima serait assurée, puisque chaque parcelle arriverait en tour d'exploitation à l'époque correspondante à son âge d'exploitabilité; les rè̀gles sur l'assiette des coupes seraient observées d'après la supposition que nous avons faite; et le rapport soutenu serait réalisé, puisque les conditions de végélation étant les mêmes pour toutes les parcelles, chaque coupe donnerait à surface égale un produit égal; enfin, la méthode d'exploitation du taillis simple consistant à couper, à blanc étoc, les peuplements destinés à être régénérés, il en résulte que l'on peut, sans contrarier l'application de cette méthode, préciser l'année et l'emplacement où chaque coupe devra être effectuée, et que les prescriptions du plan d'exploitation, à ce sujet, se concilient avec les exigences de la culture.

Ce plan ne serait cependant pas complet, si l'on 
n'y ajoutait, comme annexe, les indications nécessaires, relativement aux coupes d'amélioration (nettoiementśs) et à la réserve en baliveaux de l'âge, que l'état du peuplement ou l'intérêt dú.propriétaire pourrait réclamer.

I Les nettoiements dans les taillis n'ont, en général, aucune importance, au point de vue de leur rendement immédiat en matière ou en argent; ils en ont. une plus ou moins grande, quelquefois nulle, au point de vue cultural; il n'était donc pas nécessaire de les faire figurer dans le tableau d'exploitation qu'ils auraient compliqué sans utilité. Quant aux baliveaux à réserver, on ne saurait d'avance en préciser ni le nombre ni la grosseur.

L'aménagement d'une forêt placée dans les conditions favorables que nous avons admises serait, comme on le voit, bien facile à faire; mais ces conditions sont, on peut le dire, imaginaires. Nos forêts offrent, presque toutes, une grande irrégularité de peuplement, qui tient en partie à la nature même des choses et qui les éloigne plus ou moins de la forêttype que nous avons prise pour exemple: tantôt c'est l'âge d'exploitabilité qui n'est pas le même pour tous les massifs qu'on voudrait comprendre dans le même aménagement; tantôt c'est la gradation des âges qui est insuffisante ou qui ne s'accorde pas avec les règles d'assiette. des coupes; ou bien c'est la puissance productive du sol qui est inégale... et ces circonstances fâcheuses font du règlement d'exploitation une opération quelquefois très-délicate et très-ardue. 
Examinons successivement les principaux embarras qui peuvent se produire, et cherchons les moyens de les surmonter.

Ces embarras sont presque toujours complexes et contradictoires en ce sens qu'il est rarement possible de remédier à un inconvénient sans en faire naître un autre.

La question est donc de savoir, entre deux maux, choisir le moindre.

ARTICLE II.

CLASSEYEXT DES PARCELLES SEIVAXT L'AGE

D"EXPLOITABILITÉ.

Cette considération de l'âge d'exploitabilité est celle qui se présente la première à l'esprit, quand on veut procéder au classement des parcelles dans le tableau d'exploitation.

Rien de plus facile que de s'y conformer, lorsque l'âge d'exploitabilité est le même pour toutes les parcelles de la forêt, et qu'aucune raison ne s'oppose à ce que les peuplements que renferment ces parcelles puissent rester sur pied jusqu'à l'époque correspondante à cet âge.

Mais, l'àge d'exploitabilité étant le même pour toutes les parcelles, il arrive souvent que, par suite, soit d'une exploitation vicieuse, soit d'un abroutissement, d'une gelée ou de toute autre cause acciden- 
telle, le peuplement d'une ou plusieurs d'entre elles exige une exploitation anticipée. Les faits de ce genre sont consignés dans le cahier de la description spéciale; on doit y avoir égard, et pour cela on classe les parcelles dans les colonnes du tableau qui correspondent, non à l'âge normal d'exploitabilité', mais à l'âge où elles devront être réellement exploitées, à raison de l'état accidentellement mauvais de la végétation.

Lorsque l'âge d'exploitabilité n'est pas le même pour toutes les parcelles, on adopte pour base du règlement d'exploitation l'âge d'exploitabilité le plus reculé, et l'on partage en conséquence le tableau de classement en autant de colonnes qu'il y a d'années dans cet âge. On colloque ensuite les différentes parcelles dans ces colonnes, de manière que chacune d'elles arrive périodiquement en tour d'exploitation à l'âge qui lui convient; mais, pour cela, il faut que l'âge d'exploitabilité le plus reculé soit un multiple des autres, et, alors, on fait figurer chaque parcelle dans le tableau, aulant de fois qu'il y. a d'unités dans le quotient de cet âge d'exploitabilité par le sien. Une parcelle en châtaigniers qui serait, par exemple, exploitable à 10 ans, figurerait deux fois dans le tableau d'exploitation d'une forêt dont la révolution serait fixée à 20 ans.

Lorsque certaines parcelles ont un âge d'exploitabilité qui n'est pas renfermé un nombre exact de fois dans la révolution adoptée, elles ne peuvent être comprises dans le même aménagement, à moins qu'on 
ne se résigne à les couper avant ou après leur âge d'exploitabilité, ou qu'on ne les destine à une transformation qui les ferait rentrer dans les conditions désirables. Si un peuplement, exploitable à 15 ans, appartenait, par exemple, à une forêt dont le surplus serait exploitable à 20 , on pourrait classer ce peuplement dans le tableau d'exploitation de manière à le faire arriver en tour d'exploitation, dans la première révolution, à l'époque correspondante à son âge d'exploitabilité de 15 ans; seulement, pour le maintenir dans l'aménagement sans être forcé de modifier à chaque révolution le plan d'exploitation, il faudrait, à partir au moins de la deuxième, ou porter à 20 ans son âge d'exploitation, ou le réduire à $\mathbf{1 0}$, ou substituer à l'essence dont il se compose, une essence dont l'âge d'exploitabilité serait conciliable avec celui des autres parcelles:

La divisibilité de l'àge d'exploitabilité le plus reculé par les autres, est donc la seule condition rigoureusement indispensable pour que l'on puisse comprendre dans le même aménagement, des parcelles dont l'exploitabilité différerait; mais on verra que cette différence est souvent un obstacle à l'applícation des règles d'assiette et rend, dans tous les cas, l'établissement du rapport soutenu d'autant plus difficile, que les parcelles exploitables à l'âge le plus reculé sont moins nombreuses par rapport aux autres. Aussi, dans la pratique, adopte-t-on pour base du classement l'âge d'exploitabilité qui convient à la majorité des parcelles, et laisse-t-on en dehors 
de l'aménagement tous les peuplements exploitables à un âge plus avancé, lorqu'on croit devoir se conformer à leur âge d'exploitabilité.

Quant aux parcelles exploitables à une époque plus rapprochée, leur classement se fait comme-je l'ai indiqué.

Une fois le classement des parcelles établi d'après les considérations précédentes, il faut voir s'il est en harmonie avec les règles sur l'assiette des coupes.

\section{ARTIGLE III.}

CLASSEMENT DES PARCELLES CONFORMÉMENT AUX RÈGLES D'ASSIETTE DES COUPES.

Les parcelles de la forêt que nous avons à aménager se trouvent dans des conditions de végétation tout à fait identiques; les essences dont elles se composent, la consistance qu'elles présentent, le sol sur lequel elles reposent, les circonstances climatériques auxquelles elles sont soumises, sont les mêmes. Elles ne diffèrent que par la contenance et par l'âge; mais on remarque qu'en additionnant les contenances des parcelles de même âge, on obtient, pour chacun des âges que présente la suite des nombres de 1 à 20 , le même total.

Cet état de choses, que je suppose constaté par. le tableau d'exploitation dont j'ai donné plus haut le modèle, est-il suffisant pour constituer un état normal? 
Non, car s'il remplit deux des conditions auxquelles j'ai dit que le plan d'exploitation devait satisfaire, il n'est pas prouvé qu'il remplisse la troisième; s'il s'accorde parfaitement avec le principe qui veut que les peuplements arrivent en tour d'exploitation à l'époque correspondante à l'âge d'exploilabilité; s'il. s'accorde aussi bien avec celui qui demande que les exploitations soient distribuées dans le cour's de la révolution, de manière à procurer chaque année un produit soutenu; rien ne démontre qu'il se concilie avec les règles sur l'assiette des coupes, règles que voici ${ }^{1}$ :

$1^{\circ}$ Dans une même série d'exploitation, les coupes doivent être assises de manière à se succéder de proche en proche, et recevoir la forme la plus régulière possible.

$2^{\circ}$ Elles doivent être disposées de manière que les bois d'une coupe en exploitation ne soient pas dans le cas d'être transportés à tráver's d'autres coupes précédemment exploitées.

$3^{\circ}$ Dans toute forêt ou série d'exploitation, les coupes doivent être assises de manière que celles qui sont à exploiter au commencement de la révolution se trouvent placées du côté du nord ou de l'est. et les dernières du côté du sud ou de l'ouest.

$4^{\circ}$ En montagne, il faut couper d'abord les parties inférieures, et conserver les supérieures pour les dernières exploitations.

1. J'emprunte ces règles au Cours de culture de MM. Lorentz et Parade. 
$5^{\circ}$ Dans tous les cas, les coupes en montagne, autant que les localités le permettront, devront être longues et étroites et présenter leur moindre largeur aux vents dangereux.

Il faut donc que notre plan d'exploitation ne contrarie pas l'application de ces règles; or, en l'examinant de près, on reconnait qu'il s'y oppose sur plusieurs points : ainsi, une parcelle qui n'a que 5 ans est contiguë à une autre âgée de $\mathbf{1 5}$ ans et qui, si l'on ne modifiait pas le classement, devrait être exploitée 10 ans plus tard. Une troisième parcelle se trouve en pente immédiatement au-dessus d'une quatrième qui est moins âgée. Les parcelles les plus âgées sont situées précisément du côté du_sud d'où viennent les vents les plus violents. Enfin, aucune des coupes n'offre, sous le rapport de la forme, la régularité convenable...

Que faire en présence d'une pareille situation?

$\mathrm{Si}$ on maintient l'ordre des coupes fondé sur l'àge d'exploitabilité, il en résultera des inconvénients; si on le modifie pour le conformer aux prescriptions des règles d'assiette, ces inconvénients dis paraîtront, mais ils sèront remplacés par d'autres. Quel que soit le parti que l'on prenne, il faut d'avance se résigner à des sacrifices. Seulement, ces sacrifices peuvent être plus ou moins considérables, et c'est a les diminuer que l'on doit tendre naturellement. Je ne saurais indiquer le moyen infaillible d'arriver, sous ce rapport, au but le plus désirable. La nature des choses ne le comporte pas. Je ne peux qu'ex- 
poser les principes généraux qui me paraissent propres à éclairer le jugement de l'opérateur et à l'empêcher de faire fausse route

Les principales circonstances qui influent sur l'importance de l'application des règles d'assiette sont : la configuration du sol et le mode d'exploitation.

C'est en montagne que l'assiette des coupes réclame une attention toute particulière, parce que les accidents météoriques y sont plus nombreux et plus violents que dans la plaine, et que, d'ailleurs, la déclivité et l'escarpement du sol augmentent naturellement les difficultés de la surveillance, de l'exploitation, et de la vidange.

Mais c'est dans les futaies que la dérogation aux règles d'assiette est surtout dommageable. Dans les taillis, et dans les taillis simples notamment, les deux premières règles sont, en général, les seules qu'il soit véritablement utile d'observer; en effet, les vents, dont les trois autres ont pour objet d’atténuer la violence, ne sont guère à redouter pour le sousbois qui croît à l'état serré jusqu'au moment de son abatage; qui ñe s'élève jaınais beaucoup, et qui est toujours assez flexible pour plier, sans se rompre ou se déraciner, sous l'effort de la tempête.

Quant aux réserves, comme elles sont clair-plantées et qu'elles dominent le massif, la disposition des coupes ne saurait avoir, sur leur conservation, une influence appréciable.

Quels que soient, au reste, les motifs qui rendent 
l'application de telle ou telle règle utile et même nécessaire, il n'est pas indispensable, pour que cette application atteigne le but désirable, qu'elle assujettisse à la même loi toutes les coupes à faire durant la révolution. Il y a, dans le nombre des coupes dont on 'veut déterminer l'assiette, une limite au delà de laquelle les avantages de certaines règles disparaissent complétement. Ainsi, lorsqu'il s'agira d'appliquer la première règle, qui demande que les coupes soient assises de manière à se succéder de proche en proche, on pourra souvent, sans renoncer à aucun des avantages d'une semblable disposition, partager la révolution en périodes, et ne réaliser l'ordre régulier que pour les coupes de chacune de ces périodes, envisagée séparément.

Les règles d'assiette ne s'accordent pas toujours entre elles. Il arrive même fréquemment que l'application de l'une met obstacle à l'application de l'autre. Cette oppesition est la difficulté à laquelle il faut remédiei d'abord, en choisissant entre les deux règles qui se contrarient celle qui emprunte à la configaration du sol, à la nalure du climat, à l'état du peuplement la plus grande importance.

Il y a une règle, la deuxième, qui est indépendante de l'ordre des exploitations, en ce sens que, quel que soit cet ordre, elle peut être observée au moyen de travaux d'art plus ou moins coutteux. II faudrait que ces travaux fussent bien considérables pour qu'on s'abstînt de les faire, en présence des inconvénients qui résulteraient de l'inobservation 
mème partielle des règles d'assiette. L'intérêt du capital que lesdits travaux nécessiteraient, comparé à la perte que subirait la production annuelle, si on laissait les choses dans le statu quo, servira de guide dans les appréciations de cetle nature.

C'est à l'aide de ces principes que l'on se fixera sur l'ordre dans lequel il serait à souhailer "que les parcelles de la forêt à aménager se succédassent. et sur les changements qu'il y aurait lieu d'introduire en conséquence dans le classement de ces parcelles.

Comparons maintenant les inconvénients qui résulteraient de la non-observation des règles d'assiette à ceux que leur application entrainerait.

Le plan d'exploitalion ayant été établi d'après l'âge d'exploitabilité des différentes parties qui constituent la forêt, on ne saurait le modifier, d'une manière quelconque, sans reculer ou avancer, pour un oú plusieurs massifs, l'époque de l'exploitation, et, par conséquent, sans porter atteinte au produit le plus avantageux, sans amoindrir le revenu que la forèt eût été susceptible de procurer dans un temps donné.

Tel est le seul inconvénient qu'entraînerait la modification du classement fondé sur l'àge d'exploịtabilité.

Quelle est sa gravité? Est-elle plus grande quand on dépasse l'âge d'exploitabilité que lorsqu'on - le devance?

On comprend que ces questions ne comportent 
pas une solution rigoureusement exacte. II serait téméraire d'y répondre d'une manière absolue, soit dans un sens, soit dans l'autre.

On peut, cependant, poser en principe qu'il est presque toujours moins désavantageux de laisser les bois trop longtemps sur pied, que de les couper trop tôt; car, dans le premier cas, on ne perd guère que sur la quantité, tandis que, dans le second, on est exposé à perdre, à la fois, et sur la quantité et sur la qualité. On a vu, d'ailleurs, dans la théorie de l'exploitabilité, que le retard apporté à l'exploitation d'un bois se traduit par une accumulation de produits; si cette accumulation est fâcheuse, parce qu'elle ne rend pas l'intérêt qu'on aurait pu retirer de ces produits en les réalisant et en en plaçant le prix, elle l'est moins toutefois que ne le serait une exploitation prématurée par laquelle on compromettrait, en même temps, son capital et son revenu.

Voilà un premier principe; en voici un second :

La perte causée par une exploitation prématurée ou tardive est en raison de la différence qui existe entre l'âge d'exploitabilité et l'àge correspondant à l'époque de cette exploitation ; d'où il suit que c'est dans les bois qui sont soumis à de longues révolutions, que les difficultés de l'espèce ont le plus de gravité.

Mais, quelle que soit la perte que puissent occasionner les modifications dont je viens de prévoir l'opportunité, il faut considérer qu'elle est subordonnée à des circonstances transitoires, destinées à dis- 
paraître ordinairement après la première révolution, c'est-à-dire après le temps nécessaire pour l'établissement de l'état normal.

Les pertes qui résulteraient de la non-application des règles d'assiette tiendraient, au contraire, à des circonstances permanentes, et se reproduiraient à chaque révolution. Pour qu'il en fùt autrement, il devrait s'opérer dans la configuration du sol, dans le climat et dans les autres conditions de la végétation, des changements évidemment impossibles.

Si, par exemple, l'on n'asseyait pas les coupes de proche en proche, en ayant soin de leur donner la forme la plus convenable, on se condamnerait, pour toujours, à aggraver les difficultés de la surveillance, et les dommages inévitables causés par le couvert des arbres voisins, par l'exploitation et la vidange des produits.

Si on ne les disposait pas de manière à ne point être obligé de transporter les bois d'une coupe en exploitation, à travers d'autres coupes récemment exploitées, chaque année verrait se renouveler et s'augmenter les dégâls ruineux occasionnés par le charroi des produils, etc.

C'est là un caractère, la permanence des causes de perte, qui est de nature à peser d'un grand poids dans la balance des inconvénients qu'entraînent, d'une part, la dérogation aux règles d'assiette, et, de l'autre, l'exploitation soit avant, soit après l'âge d'exploitabilité: 
Ayant à choisir entre une perte de produits, accidentelle et temporaire, et un dommage susceptible de se renouveler indéfiniment, le choix ne saurait être douteux, et le simple bon sens veut qu'on prenne les dispositions nécessaires pour faire disparaître la cause du dommage, sauf à ménager la transition de l'état actuel à l'état désirable, de façon à répartir sur un plus grand nombre d'années, la perte de produits inhérente à une exploitation tardive ou prématurée.

On y arrivera, en ne modifiant qu'en partie la marche des coupes pour les premières révolutions.

Exemple. Il s'agit d'un bois partagé en vingt coupes, situé en pente rapide, dont les exploitations ont toujours été faites, jusqu'à présent, de bas en haut, mais dans lequel, l'impossibilité de construire des chemins de vidange, impose à l'adjudicataire la nécessité de transporter les bois de la coupe en exploitation, à travers d'autres coupes précédemment exploitées.

Le maintien d'un pareil état de choses est inadmissible. Il faut renverser l'ordre des coupes; mais on ne peut songer à effectuer ce changement sans transition, puisque de la coupe exploitable on tomberait dans les bois d'un an. On prendra donc les dispositions suivantes :

Pendant la première révolution, les cinq premières coupes seront assises dans les bois âgés actuellement de 16 à 20 ans; les cinq autres, dans les bois de 11 à 15 ans; les cinq suivantes, dans les bois de 6 à 
10 ans, et les cinq dernières, dans les bois de 1 à 5 ans.

Un an après la première révolution, les cinq coupes situéeś à la partie supérieure de la pente, auront, en commençant par la plus élevée, de $\mathbf{5}$ ans à 1 an; les cinq autres coupes auront de 10 à 6 ans; les cinq suivantes, de $\mathbf{1 5}$ à $\mathbf{1 1}$ ans, et les cinq plus basses, de 20 à 16 ans.

Pendant la deuxième révolution, on cominencera les exploitations par la coupe âgée de $\mathbf{1 5}$ ans, et on les continuera en descendant successivement jusqu'à la coupe inférieure, qui, alors, aura $\mathbf{2 5}$ ans; puis, on se portera à la partie supérieure où l'on trouvera des bois de 15 ans, et on descendra successivement jusqu'au milieu de la pente où l'on terminera l'exploitation par des bois de 25 ans...

A partir de la troisième révolution, l'ordre définitif sera suivi, et, à partir de la quatrième, chaque coupe sera exploitée à l'époque correspondant précisément à son âge d'exploitabilité. Le maximum de la différence entre cet âge et celui où aura lieu l'exploitation, sera de $\mathbf{5}$ ans, tantôt en plus, "tantôt en moins, pendant trois révolutions. Cela n'a rien d'exorbitant.

J'ai supposé un cas extrême et par conséquent très-rare. La formation du plan d'exploitation des taillis n'exige pas ordinairement ces ménagements et ces prẻcautions, dont je viens de donner des exemples. Toutes les fois que cette formation n'impose que des transpositions de parcelles d'une faible éten- 
due ou le redressement des contours des coupes, les avantages qu'on réaliserait, en appliquant à l'opération les mesures transitoires indiquées précédemment, ne vaudraient pas ceux que procurerait l'établissement immédiat de l'état définitif.

Voilà pourquoi on se borne le plus souvent, dans les aménagements de taillis, à partager la forêt en coupes parfaitement régulières qu'on exploite ensuite, de proche en proche, conformément aux règles d'assiette, en s'efforçant, il est vrai, autant que possible, de ne pas trop s'éloigner de l'àge d'exploitabilité, mais en considérant toutefois comme secondaires, les difficultés que rencontrerait l'accomplissement de cette dernière condition.

Dans tous les cas, et quand bien même on croirait devoir adopter, un plan d'exploitation provisoire, il n'en faudrait pas moins arrêter le plan dêfinitif, le plan normal, et c'est même la première opération à laquelle il conviendrait de procéder. Ce plan n'en serait pas moins appliqué sur le terrain, et.le plan provişoire ne figurerait que sur le papier, sous la forme d'un état d'assiette motivé.

On connaît maintenant la plupart des obstacles qui peuvent s'opposer à l'observation des règles d'assiette, et l'on voit qu'il n'y en a pas d'insurmontables. Cependant il en est un dont je n'ai pas parlé et qui le serait : c'est celui qui consisterait dans l'existence, au milieu d'un bois exploituble à un certain àge, d'une parcelle dont l'exploitabilité serait différente et qui devrait, en conséquence, arriver en 
tour d'exploitation, deux ou plusieurs fois dans le cours de la même révolution. Il est certain que cette parcelle ne pourrait être placée dans les conditions désirables, de végétation, de vidange, et de surveillance. Aussi, est-ce là un motif de plus pour que l'on ne inêle pas, dans le même aménagement, des bois exploitables à des âges différents.

Je l'ai déjà dit et je le dis encore en finissant, car je crois que cela est de la plus grande importance : l'application des deux premières règles d'assiette est de rigueur. J'insiste sur ce principe, parce qu'on semble le méconnaître généralement.

Les dommagres causés par le couvert des bois voisins; ceux qui résultent de l'inefficacité de la surveillance rendue plus difficile; les frais qu'occasionne le réarpentage des coupes ou la recherche des lignes séparatives sinueuses; les dégâts mêmes occasionnés par la vidange; tous ces inconvénients ne paraissent pas bien graves quand on les considère séparément; ils passent inaperçus, et, cependant, ils se traduisent finalement par une diminution, beaucoup plus forte qu'on ne croit, du produit net.

C'est surtout en matière d'exploitation forestière qu'il n'y a pas de petiles économies. 


\section{ARTICLE IV.}

CLASSEMENT DES PARCELles CONFORMÉment AUX EXIGencrs DU RAPPORT ANNUEL SOUTENU.

\section{$\$ 1$ er.}

Conditions nécessaires pour que le rapport annuel soutenu puisse etre réalisé.

Pour que l'on puisse retirer d'une forêt un rapport annuel et soutenu, il faut, de toute nécessité, deux choses :

$1^{\circ}$ Que les âges des différentes parcelles dont cette forêt se compose, soient gradués de manière qu'il y ait possibilité de livrer chaque année à l'exploitation, une portion du peuplement, sans s'imposer de trop grands sacrifices sur l'accroissement ;

$2^{\circ}$ Que chacune de ces portions soil susceptible de fournir, à l'époque fixée pour son exploitation, le même produit et occupe, en conséquence, une étendue inversement proportionnelle à sa puissance productive comparée à un terme commun.

Établissons d'abord dans quels cas et à quelles conditions le rapport annuel est possible : nous chercherons ensuite les moyens de rendre soutenu ce rapport annuel.

Pour l'intelligence des principes-relatifs, soit à l'âge d'exploitabilité, soit à l'assiette des coupes, il 
n'était pas indispensable de supposer, comme nous l'avons fait dans les chapitres précédents, que la gradation des âges était parfaitement régulière et ne présentait pas de lacune. Ces principes trouvent en effet leur raison d'être, dès que l'on adınet qu'il existe dans une forêt des massifs dont les âges diffèrent el qui demandent, par conséquent, à ne pas être exploités à la même époque.

La réalisation du rapport annuel exige, au contraire, qu'il y ait dans les âges une gradation, sinon parfaitement régulière et complète, telle, du moins, que ses défectuosités s'arrêtent à de certaines limites.

Quelles sont ces limites?

Nous continuerons à nous maintenir dans l'hypothèse que l'âge d'exploitabilité de la majorité des parcelles est de 20 ans.

Nous : classons ces parcelles dans. les diverses colonnes du plan d'exploitation, et nous ne trouvons rien à porter dans les dix premières colonnes, ce qui montre que la forêt ne renferme pas de bois âgés de plus de 10 ans.

Si l'on voulait, dans cet état de choses, réaliser immédiatement le rapport annuel, il faudrait se résigner à exploiter des bois de 10 ans, et à perdre, en conséquence, le bénéfice de l'accroissement des 10 dernières années. Ce sacrifice serait considérable, et il est d'autant moins admissible que l'on consente à le faire, que des boìs de $\mathbf{1 0}$ ans n'ont, d'ordinaire, qu'une très-faible valeur vénale. On attendra, pour 
commencer les exploitations, que l'on ait des peuplements plus âgés.

Donc, une première condition pour qu'une forêt soit immédiatement productive d'un rappórt annuel, c'est qu'elle renferme des bois exploitables ou assez près de l'être, pour qủe l'on puisse en effectuer l'exploitation, sans subir une trop grande perte d'accroissement.

Au lieu de ne renfermer que des bois au-dessous de 10 ans, la forêt n'en renferme que de 15 à 20 ans.

Il est clair que si l'on ne voulait consentir à aucune perte sur l'accroissement, l'aménagement ne serait pas plus possible dans ce cas que dans le précédent, puisque après 5 ans, il n'y aurait plus de bois à exploiter; la forêt se trouverait transformée en un jeune taillis de $\mathbf{1}$ à 5 ans; les coupes devraient être forcément interrompues.

Admettons que l'on tienne beaucoup à établir immédiatement le rapport annuel. Pour cela, il sera nécessaire de n'exploiter qu'en 20 ans le peuplement existant, et, dès lors, de laisser sur pied pendant encore 20 ans, des bois qui en ont déjà 15. Ces bois auraient, à l'époque de leur abatage,' 35 ans, soit 15 ans de trop. Il est probable qu'un âge aussi reculé ne saurait convenir ni à l'intérêt bien entendu du propriétaire, ni aux exigences de la reproduction.

Donc, une deuxième condition pour qu'une forêt soit productive d'un rapport annuel, c'est qu'outre des bois exploitables ou sur le point de l'être, elle en 
contienne d'assez jeunes pour que l'on puisse, sans de trop grands inconvénients, les laisser sur pied jusqu'à la fin de la révolution.

La forêt renferme des bois de 2 ans, de 20 ans, et de quelques autres âges intermédiaires; mais les bois de 3 à 12 ans manquent complétement. Pour assurer la continuité des produits, il faudrait remplir ces lacunes et remplacer les bois de 3 à 12 ans, absents, par une partie de ceux de $\mathbf{2}$ ans et une partie de ceux de 13 , et exploiter les uns 5 ans avant l'âge d'exploitabilité, les autres 5 ans après. Lorsque les vides à remplir sont trop grands, ils s'opposent à l'obtention immédiate du rapport annuel.

Donc, une troisième condition pour qu'une forèt soit productive d'un rapport anniel non interrompu, c'est que la différence d'âge entre les peuplements dont les exploitations doivent se suivre, soit telle qu'en la partageant en deux, on ait un nombre d'années qui ne dépasse pas le maximum de l'écart que l'on est disposé à tolérer, entre l'ấge d'exploitabilité et l'âge correspondant à l'époque de l'exploitation.

Telles sont les conditions auxquelles le rapport annuel est subordonné, lorsque la révolution définitive est immédiatement applicable.

Si l'on était forcé d'adoptèr une révolution principale transitoire, il faudrait, en outre, que cette révolution fût assez longue pour que les parties de la forêt qui auraiẹnt été régénérées à son début, pussent être de nouveau exploitées à son expiration. 
En résumé, le défaut de gradation dans les àges d'une forêt, peut être tel qu'il empêche absolument l'annualité du rapport; il peut être tel que cette annualité ne soit possible qu'au moyen d'une révolution transitoire. Il peut enfin ne pas être assez grand pour s'opposer à ce qu'elle se concilie avec la révolution définitive.

Nous avons vu que l'application des règles d'assiette avait souvent pour résultat de faire modifier le tableau d'exploitation, ce qui entraînait la nécessité d'avancer ou de retarder l'abatage des peuplements dont le classement était changé. La réalisation du rapport annuel expose aux mêmes inconvénients.

Nous avons vu que, dans le classement suivant les règles d'assiette, pour diminuer, autant que possible, la perte d'accroissement occasionnée par la non-exploitation à l'àge d'exploitabilité, on pouvait être réduit à adopter un état d'assiette, c'est-à-dire un plan provisoire d'exploitation. C'est un expédient auquel on a recours quelquefois pour réaliser le rapport annuel, quand la révolution est définitive, et qu'il faut nécessairement employer, quand elle est transitoire.

Il résulte de ce qui précède :

Que lorsque le tableau d'exploitation, dressé tout d'abord d'après l'âge d'exploitabilité, a été ensuite successivement modifié : une première fois, pour qu'il se concilie avec les règles d'assiette; et, une seconde fois, pour qu'il présente une succession, non interrompue, de coupes annuelles, on peut avoir 
à assurer le rapport soutenu dans les circonstances suivantes :

$1^{\circ}$ Révolution principale définitive; marche des coupes, normale.

$2^{\circ}$ Révolution principale définitive; marche des coupes, provisoire.

$3^{\circ}$ Révolution principale transitoire; marche des coupes, provisoire.

\section{2.}

- Du rapport soutenu dans l'hypothèse d'une révolution princıpale définitive et d'une marche des coupes, normale.

Le classement des parcelles dans le tableau d'exploitation est terminé. Chacune des colonnes de ce tableau présente au total, une égale contenance; le rapport annuel est assuré; le rapport soutenu ne le serait que si les conditions de végétation étaient les mêmes pour toutes les coupes: or, elles sont, au contraire, très-variables. Comment s'y prendra-t-on pour y remédier?

On a imaginé, à cet effet, plusieurs moyens que je vais passer en revue; je dirai ensuite dans quelle mesure leur emploi me paraît admissible.

1. Des moyens de remédier à l'inégalité des conditions de la végélation.

1. On calcule le produit, par hectare, que don- 
nera chaque parcelle au moment où elle arrivera en tour d'exploitation, et on le compare au produit moyen par hectare de la forêt. On obtient, ainsi, pour chaque parcelle, un rapport qui exprime de combien sa puissance productive est supérieure ou inférieure à la puissance productive moyenne, et qui indique, en mème temps, ce qu'il serait nécessaire d'enlever ou d'ajouter à un hectare de cette parcelle, pour en obtenir un produit égal à celui de l'hectare pris pour terme de comparaison.

Le chiffre qui exprime le rapport par hectare entre le produit d'une parcelle quelconque et le produit moyen, est donc un coefficient par lequel il suffit de multiplier la contenance réelle de cette parcelle, pour connaître à quel nombre d'hectares d'une puissance productive moyenne équivaut cette contenance réelle.

Ce nombre est substitué dans le tableau de classement à la contenance réelle. On modifie en conséquence les totaux des colonnes, et s'ils sont inégaux, on y remédie en transportant, de proche en proche, et conformément aux principes exposés précédemment, l'excédant des colonnes trop riches dans celles qui ne le sont point assez. Ces transferts en entraînent nécessairement d'autres lorsqu'on veut opérer rigoureusement; car ils ont pour résultat d'avancer ou de retarder l'époque de l'exploitation des parcelles déclassées. On tient compte de cette circonstance, et, par des tâtonnements, par des remaniements successifs, on parvient à rendre les contenances 
des coupes, inversement proportionnelles à leur puissance productive.

Exemple: Le produit moyen par hectare $x$ est représenté par l'unité. On trouve que, calculé pour l'époque de son exploitation, le produit de la parcelle A sera de 50 pour 100 plus fort que le précédent. II est donc représenté par le chiffre 1,50. On multiplie la contenance de la parcelle, qui est de 8 hectares, par 1,50; on obtient 12 hectares. On substitue ces 12 hectares aux 8 hectares portés dans le tableau de classement. Après avoir opéré de cette manière pour les autres parcelles, on constate que la colonne, dans laquelle figure la parcelle $\mathbf{A}$, est trop riche et qu'il faut 'transporter une-portion de cette parcelle dans la colonne voisine. C'est ce que l'on fait; mais ce transfert modifie nécessairement la puissance produetive, par hectare, de la portion déclassée, puisque cette dernière ne ssra pas exploitée à l'époque que l'on avait fixée primitivement. On tient compte de cette circonstance, en réduisant ou en augmentant proportionnellement la contenance fictive de ladite portion, etc., etc.

La principale difficulté dans l'application de ce procédé, porte sur l'évaluation du volume que les parcelles présenteront, quand arrivera le moment de les exploiter. Pour faire cette évaluation, il n'y a que deux moyens : calculer, d'après l'accroissement passé, l'accroissement futur, et ajouter l'un à l'autre; ou bien, adopter comme devant être le volume qu'acquerra cette parcelle, celui d'un peuplement qui serait 
placé dans de semblables conditions de végétation, et qui aurait l'âge fixé pour son exploitation.

Le calcul de l'accroissement, d'après la mar'che antérieure de la végétation, est impossible dans les taillis, par beaucoup de motifs qu'il est inutile de développer ici, et, entre autres, parce que leur accroissement annuel suit ordinairement, jusqu'à l'époque de leur abatage, une progression qui ne permet pas de préjuger, avec quelque chance de succès, le volume qu'ils acquerront par celui qu'ils ont acquis.

C'est donc par la comparaison avec des massifs situés dans les mêmes conditions de végétation, que l'on peut espérer de parvenir au but de ses recherches. Cette opération est plus ou moins difficile, suivant l'état des lieux et les caractères plus ou moins variés des parcelles que l'on envisage. Les exploitations effectuées dans la forêt même dont on règle l'aménagement, suppléent quelquefois aux termes de comparaison que la localité n'offre pas. S'il existail des données expérimentales sur l'accroissement annuel des différentes essences, suivant les sols, les climats et les modes d'exploitation, il suffirait de recourir à ces données pour obtenir les renseignements désirables. Malheureusement elles n'existent pas.

$2^{\circ}$ Le second moyen qui a été conseillé poul' rendre le rapport soutenu, consiste dans l'appréciation directe des éléments mêmes de la végétation, c'est-à-dire, des circonstances qui sont de nature à exercer une influence sur la production. On fait, pour 
ces éléments, les mêmes calculs que pour les produits présumés des parcelles, et on obtient de cette manière les coefficients qui servent à fixer les contenances qu'il faut donner à chaque coupe, pour en retirer le même produit.

On prend par exemple, pour terme de comparaison, un massif situé dans des conditions moyennes de végétation. On exprime par l'unité l'étát du peuplement et la fertilité du lieu d'habitation. On compare à celte fertilité et à cet état de peuplement, la fertilité et l'état du peuplement de chaque parcelle. On obtient ainsi, pour chaque parcelle, deux facteurs. On multiplie ces deux facteurs l'un par l'autre; le produit exprime précisément dans quel rapport se trouve la puissance productive de la parcelle que l'on considère, avec la puissance productive du massif choisi pour terme de comparaison.

Exemple : La parcelle $\boldsymbol{A}$ renferme un peuplement dont l'état est de un quart moins satisfaisant que celui du peuplement moyen pris pour type. Son terrain, son climat, son exposition dénotent une fertilité qui, comparée à celle du peuplement moyen précité, est moins bonne de un cinquième. Il en résulte que son coefficient de puissances productive est, pour l'état de peuplement, de 0,75 , et pour la fertilité du lieu d'habitation de 0,80. En multipliant ces deux rapports l'un par l'autre, on trouve au produit 0,60 . Cela signifie que six dixièmes d'hectare ou soixante ares d'un peuplement, placés dans des conditions ordinaires de végétation, équivalent, en puissance 
productive, à un hectare de la parcelle $\mathrm{A}$, ct que pour rendre, par conséquent, la contenance de celte parcelle inversement proportionnelle à sa puissance productive, il faut la porter sur le tableau de classement pour une contenance fictive ou réduite, égale ì son étendue réelle multipliée par le coefficient 0,6 .

Des deux procédés que je viens d'exposer, quel est le meilleur?

C'est se que j'ai maintenant à apprécier.

Pour peu qu'on y refléchisse, il est facile de reconnaitre que celui dont on a parlé en dernier lieu ne constitue pas une méthode particulière; qu'envivisagé d'une manière absolue et en soi, il serait même dépourvu de toute valeur pratique, el qu'il ne peut servir qu'à compléter et à contrôler les renseignements sur lesquels, par le premier procédé, on base ses appréciations:

Tous les deux reposent d'ailleurs, évidemment, sur le même principe. Le second implique comme l'autre, que l'on connaît la production possible d'un peuplement, à un âge et dans des conditions de végétation donnés; et lorsqu'on compare entre eux les éléments de cette végétation, un terrain à un autre terrain, un état de peuplement à un autre état de peuplement, on a nécessairement, pour éclairer cette comparaison, une idée plus ou moins nette des résultats que ces éléments seraient susceptibles de fournir à des époques déterminées. Sans cette idée indispen- sable, la comparaison n'aurait pas de conclusion possible. Un terrain n'est préféré à un autre, que parce 
que l'on présume qu'il rapportera davantage. Un ẻtat de peuplement est plus ou moins satisfaisant par des raisons analogues. Dans cette matière, comme dans toutes celles où il s'agit d'apprécier la valeur relative des agents physiques, on ne peut juger des causes que par leurs effets.

Le second procédé n'échappe donc pas à la difficulté que présente l'estimation du volume futur. II rend, au contraire, cette difficulté plus grande en la compliquant, et il a, sous ce rapport, des exigences auxquelles il est impossible de satisfaire. Ainsi, il demande que l'on détermine l'influence particulière de chacun des éléments de la végétation sur le résultat futur plus ou moins éloigné de cette végétation; il demande que l'on fasse, dans ce résultat, la part qui revient, soit_à la fertilité du lieu d'habitation, soit à l'état du peuplement. Il implique, dẻs lors, que l'on trouvera autant de massifs placés dans des conditions spéciales qu'il y a d'éléments divers spécifiés. Cela n'est pas admissible; et cependant il faut remarquer que, dans l'exposé du procédé en question, on a partagé les éléments de la végétation en deux catégories seulement : l'une comprenant les circonstances relatives au peuplement, l'autre celles qui concernent la fertilité du lieu d'habitation. Les difficultés ou plutôt les impossibilités pratiques de ce procédé, dans l'état actuel de nos forêts, seraient bien plus manifestes, si l'on voulait former les coefficients de production en fonction de chacune de ces circonstances. 
Le second procédé n'a pas, en définitive, de valeur propre, puisqu'il ne supplée pas au premier. Il est impraticable, puisqu'il exige des appréciations que la nature des choses ne comporte pas. Son mérite est d'éclairer et d'assurer la marche de l'opérateur qui se propose de déterminer les rapports entre les puissances productives de divers peuplements. Ainsi, il l'oblige à apporter dans ses reconnaissances de la méthode et de la précision; il lui apprend à contrôler, par des rapprochements nombreux, les résultats d'un premier examen, en vérifiant si les différences dans les productions qui seraient indiquées, soit par des exploitations antérieures, soit par des tables d'expérience, s'accordent avec celles que présenteraient les éléments de la végétation.

A tous ces points de vue, l'examen attentif des circonstances qui sont de nature à exercer une influence sur la production, est d'un grand secours dans les opérations très-délicates auxquelles l'établissement du rapport soutenu donne lieu.

$3^{\circ}$ Indépendamment des deux moyens sur lesquels je me suis appesanti, un peu longuement peutêtre, au gré de mes lecteur's, on a appliqué à l'établissement du rapport soutenu, un expédient qùe je dois faire connaître, ne fùt-ce que pour fournir une nouvelle preuve de l'importance que certains auteurs attachent à l'égalisation des produits annuels.

On calcule, comme on le fait dans les autres méthodes, le volume de chaque coupe pour le moment de son exploitation; puis on partage la révolution en 
un cerlain nombre de périodes égales qui correspondent, chacune, à un même nombre de coupes; et. cnfin, au lieu de fixer sur le terrain les limites des coupes annuelles, on subordonne leur étendue, pendant chaque période, au volume qu'elles présenteront en bloc quand elles arriveront en tour d'exploitation, cc qui veut dire que, chaque année, on exploite la quantité de bois qu'indique le quotient du volume total présumé d'une période par la durée de cette dernière.

Ce moyen n'est pas affranchi des incertitudes d'appréciation qu'offrent les précédents; il y ajoute au contraire des chances d'erreur; il complique l'exploitation, il en compromet la régularité, et ce, sans compensation, car de deux choses l'une : ou bien les exploitations annuelles se renfermeront précisément dans le's limites qu'auraient eues les coupes, si on leur avait donné des contenánces proportionnelles à leur puissance productive, ou bien elles s'en éloigneront. Dans le premier cas, les précautions qui caractérisent le procédé en question auront été inutiles; dans le second cas, qui est infiniment plus probable, une inévitable confusion se mettra dans l'assiette des coupes, et, pour réaliser le rapport soutenu pendant quelques années, on se condamnera à des anticipations ou à des déficits successifs qui ne tarderont pas à l'altérer profondément. 
2. Des limites dans lesquelles il paraît convenable de renfermer l'emploi des coefficients de produc-. tion.

Il ne faut pas se dissimuler que dans l'état d'irrégularité où sont généralement les forêts de notre pays, et en présence du très-petit nombre de renseignements positifs que l'on a recueillis sur l'accroissement et la longévité des massifs, la réalisation du rapport soutenu, par la méthode des contenances réduites, est fort chanceuse. Il est donc prudent de ne l'essayer que dans des circonstances bien accusées et avec une grande circonspection.

Entrons, à ce sujet, dans quelques développements, cherchons quelques principes : nous les trouverons en nous rendant un compte exact de tous les éléments qui sont compris, comme données plus ou moins essentielles, dans le problème à résoudre, problème qui consiste à déterminer la puissance productive d'une parcelle de bois.

A quoi tient cette puissance productive? à quelles circonstances est-elle subordonnée?

Elle est subordonnée :

$1^{\circ} \mathrm{Au}$ temps, c'est-à-dire à l'âge qu'aura le massif quand il arrivera en tour d'exploitation;

$2^{\circ}$ A l'ẹtat du peuplement, c'est-à-dire à la nature des essences, à leur consistance, à leur végétation;

$3^{\circ}$ A la qualité du sol; 
- $\dot{4}^{\circ} \mathrm{Au}$ climat, et, principalement, à la situation et à l'exposition.

Ainsi, le produit d'une parcelle, parvenue à l'âge fixé pour son exploitation, est la résultante des actions combinées de ces diverses causes.

C'est là ce qui complique la recherche des coefficients de production. C'est là ce qui rend difficile et sourent impossible le choix des peuplements indispensables pour la détermination de ces coefficients; et le problème serait en conséquence simplifié d'autant plus, qu'il y aurait à tenir compte d'un moins grand nombre des circonstances précitées.

Or, parmi ces circonstances, les unes sont accidentelles et temporaires, tandis que les autres sont permanentes. Les unes sont susceptibles d'un effet dont le caractère favorable ou défavorable peut être prévu avec une approximation suffisante, tandis que les autres sont susceptibles de se modifier et de ne produire aucun des résultats qu'on en attendait.

L'époque de l'exploitation, lorsqu'elle ne correspond pas à l'âge d'exploitabilité, est une circonstance temporaire; elle disparaîtra presque toujours à la deuxième révolution; mais son effet peut être prévu avec assez de certitude.

Le mauvais état de la consistance ou de la végétation, s'il résultail des vices de la culture ou d'un événement fortuit, serait encore une circonstance temporaire, dont, en outre, dans la plupart des cas. l'influence ne saurait être préjugée; car il arrive souvent qu'un massif dont la consistance et la végétation 
ne sont pas ce qu'elles devraient être, se complète et répare le temps perdu, avant d'arriver en tour d'exploitation.

Quant aux circonstances qui consistent dans la nature des essences, dans l'âge d'exploitabilité, dans la qualité du sol, dans le climat, elles sont à la fois permanentes et susceptibles d'un effet qu'il est difficile sans doute de préciser, mais dont il est possible de prévoir avec certitude le caractère.

On déduit, de ces observations, les principes suivants :

Les contenances inversement proportionneiles à la puissance productive des parcelles, ne sont presque jamais admissibles, lorsqu'elles n'ont d'autre motif que la nécessité d'exploiter, pendant une révolution, certaines parcelles, soit avant, soit après l'âge d'exploitabilité. Elles ne le sont pas davantage, quand la différẹnce, dans les productions présumées, tient ì un état de consistance ou de végélation dont les causes sont fortuites, indépendantes des conditions naturelles dans lesquelles est placée la forêt.

Il y a deux raisons pour que l'on ne tienne pas compte de ces circonstances :

La première, c'est qu'il est, en général, comme on vient de le faire observer, difficile de déterminer leur influence sur la production; la seconde, c'est qu'en admettant qu'on y parvînt, on se condamnerait à modifier de nouveau les contenances des coupes après la première révolution, chose que l'on doit éviter autant que possible. 
Les caractères essentiels et permanents, qui dérivent de l'exploitabilité, de la nature des essences, de la qualité du terrain et du climat, sont les seuls qu'il convienne ordinairement de prendre en considération, et encore faut-il qu'ils soient bien prononcés:

Si dans nos 200 hectares de taillis exploitables, presque tous, à 20 ans, nous avions des parcelles dont l'âge d'exploitabilité serait de 10 ans, il y aurait tout lieu de croire que ces parcelles ne fourniraient pas, par hectare, le même produit que le surplus de la forèt; il serait donc nécessaire, pour réaliser le rapport soutenu, d'augmenter proportionnellement l'étendue des coupes dont elles feraient partie.

La diversité des essences, toutes les autres circonstances étant égales d'ailleurs, pourrait justifier une mesure analogue : ainsi, un taillis de châtaignier exploité à 20 ans, fournirait probablement, par hectare, un plus grand produit qu'un taillis de chêne que l'on couperait au mème âge.

Voici des parcelles dont le sol est très-mauvais. L'expérience a prouvé que, dans un temps donné, la production par hectare de ce taillis est à peine égale aux troits quarts de celle du surplus de la forêt. Ce n'est certainement pas avec le maintien du mode d'exploitation en taillis, que le sol pourra jamais s'améliorer; et, dès lors, un coefficient de fertilité est applicable à ces parcelles.

En ce qui concerne le climat, c'est surtout l'influence de l'exposition qu'il importe de ne pas négliger, 
lorsqu'on veut rendre les contenances des coupes inversement proportionnelles à leur puissance productive. Un taillis, situé sur une pente méridionale, sera évidemment beaucoup moins productif qu'un taillis exposé au nord.

Mais, je ne me lasserai pas de le dire, l'emploi des coefficients de production dans la fixation des contenances des coupes annuelles, ne doit avoir lieu que dans des cas exceptionnels. Il n'y faut recourir que loŕsque les différences dans les conditions de la végétation sont bien tranchées, et portent sur des étendues assez considérables, pour que la compensation entre le bon et le mauvais soit impossible dans" la même coupe. Il n'y faut recourir encore que lorsque l'établissement du rapport soutenu n'est pas réalisable par le moyen plus rationnel et plus sûr de la division préalable de la forêt en séries ${ }^{1}$.

\section{§3.}

Du rapport soutenu dans l'hypothèse d'une révolution principale définitive et d'une marche des coupes, provisoire.

Dans l'article sur la formation du tableau des exploitations, conformément aux règles d'assiette, j'ai montré qu'il était convenable, pour les taillis, quelles que dussent être d'ailleurs les coupes anormales qu'exigerait, pendant plus ou moins longtemps,

1. Voir pour les séries la quatrième étude. 
I'état du peuplement, d'arrêter l'ordre définitif des coupes et de l'établir sur le terrain.

Dans ce que nous venons de faire observer, relativement aux exigences du rapport soutenu, il n'y a rien qui soit de nature à détruire celte convenance. Nous avons reconnu, en effet, qu'il n'y avait pas lieu de se préoccuper de l'influence des circonstances accidentelles et temporaires sur le rendement des coupes; or les circonstances qui motivent l'adoption d'un plan provisoire sont nécessairement toujours accidentelles et temporaires, et, par conséquent, les contenances respectives des coupes doivent être fixées comme elles le seraient, si la rotation normale pouvait être immédiatement appliquée.

\section{\$ 4.}

Du rapport scutenu dans l'hypothèse d'une révolntion principale transitoire et d'une marche des coupes, prorisoire.

Les circonstances qui réclament une révolution transitoire, sont accidentelles comme celles qui réclament un plan provisoire, et ne sauraient dès lors justifier une division de coupes qu'il faudrait nécessairement modifier à l'expiration de cette révolution transitoire.

Le nombre, la contenance et l'ordre des coupes seront donc, comme dans le cas précédent, fixés sur le terrain conformément aux exigences de l'état normal. 
On pourra toutefois, dans le plan ou plutòt l'état d'assiette provisoire qu'on dressera pour la première révolution, ajouter arbitrairement à la coupe réglementaire une contenance plus ou moins grande, selon la consistance du peuplement.

Il faut remarquer que la révolution transitoire ne permettant pas de renfermer les coupes à faire durant cette révolution, dans les limites normales, il n'y a pas de raison d'ordre qui demande qu'on fasse taire absolument devant elle les convenances du rapport soutenu, l'intérêt de l'égalisation des produits annuels.

ARTICLE V.

RÉSUMÉ ET CONCLUSION.

Résumons-nous :

L'abatage à l'âge d'exploitabilité,

L'application des règles sur l'assielte des coupes,

La réalisation d'un rapport annuel et soutenu,

Tels sont les résultats que l'on doit se préoccuper d'assurer dans la formation du plan d'exploitation du taillis simple.

Plusieur's causes peuvent s'y opposer :

$\mathbf{1}^{\circ} \mathrm{La}$ situation relative des parcelles d'àges différents;

. $2^{\circ}$ La différence des âges d'exploitabilité des peuplements de ces parcelles;

$3^{\circ}$ Le défaut de gradation dans les âges; 
$4^{\circ}$ L'inégalité dans, les puissances productives des parcelles.

Les deux premières causes contrarient l'obtention des deux premiers résultats; le fàcheux effet de l'une d'elles, la seconde, est irrémédiable : lorsque l'exploitabilité n'est pas la même pour toutes les parcelles, il n'est pas possible de les faire arriver en tour d'exploitation à l'époque correspondant à cet àge, sans s'écarter des prescriptions des règles d'assiette, et vice versd; aussi, doit-on s'abstenir de comprendre dans le mème aménagement des peuplements exploitables à des âges différents. Quant aux obstacles qui proviendraient de la situation relative des parcelles, on les surmonte en se condamnant a une perte temporaire d'accroissement; et comme, dans les taillis, la révolution n'est pas assez longue pour que cette perte soit jamais hien considérable, on néglige ordinairement de la répartir sur plusieurs révolutions, à moins que l'intérèt de la reproduction ne le réclame.

Les autres causes qui intéressent particulièrement le rapport soutenu, exigent aussi des sacrifices d'accroissement : ainsi, pour rétablir dans les âges la gradation convenable, il est nécessaire d'exploiter, une ou plusieurs fois, un nombre plus ou moins grand de parcelles, soit avant, soit après l'àge d'exploitabilité; mais, par la raison que je viens de donner, il est rare que l'on ne puisse pas, à cause des pertes qui en résulteraient, procéder immédiatement à l'établissement de l'ordre définitif. 
Pour ce qui est de l'inégalité dans les puissances productives des parcelles, elle tient à plusieurs circonstances plus ou moins dignes d'attention, et qui se partagent en deux catégories bien distinctes:

Les unes sont accidentelles et temporaires, les autres sont permanentes.

Si l'on tenait compte des premières, on nuirait infailliblement à la régularité des exploitations, laquelle est très-importante à obtenir; on retarderait la réalisation de l'état normal, résultat non moins désirable. On ne doit donc, en principe, attacher de l'importance qu'aux différences qui porteraient sur la nature des essences, sur leur exploitabilité, sur la qualité du sol, sur la nature du climat, et qui seraient susceptibles d'entrainer des variations sensibles dans les produits annuels. Pour les taillis, ces variations sensibles se présentent rarement, et quand elles se présentent, la division de la forêt en séries ${ }^{1}$ permet presque toujours d'y obvier et de se dispenser des calculs très-incertains que nécessiterait la détermination de contenances inversement proportionnelles à la puissance productive.-De sòrte que, en définitive :

Lorsqu'on veut régulariser l'exploitation d'une forêt traitée en taillis simple, on se borne ordinairement à la partager en coupes régulières, d'égales contenances, exploitables de proche en proche, dans le sens prescrit par les règles d'assiette.

C'est en cela que consiste l'opération essentielle

4. Voir la quatrième étude. 
de l'aménagement, et mes lecteurs, qui ne l'ignorent pas, auront été peut-être surpris des longs développements et des hypothèses plus ou moins vraisemblables, dont j'ai fait précéder cette conclusion; ils se seront dit plus d'une fois : Est-ce que l'on se donne tant de peines pour former le plan d'exploitation d'un taillis? est-ce qu'on y met tant de façons?

Je dois répondre à ces objections :

Les diverses combinaisons que j'ai exposées ne sont point restées à l'état de pures spéculations; on les a pratiquées, on les pratique encore, même dans les taillis. Toutefois, leur utilité, pour les peuplements de ce genre, est souvent contestable, et si, malgré cela, je les ai étudiẻes avec une attention qui peut, au premier abord, paraître superflue, c'est parce que je me préoccupais de leur application dans les forêts soumises aux autres modes de culture. En traitant de l'aménagement des futaies, nous retrouverons les exigences que nous avons rencontrées dains celui des taillis. Nous les retrouverons agrandies, et nous reconnaitrons que pour y satisfaire, dans la formation du plan d'exploitation, il est souvent nécessaire de recourir aux combinaisons dont il s'agit.

Parmi les suppositions qui auront probablement le plus étonné ceux de mes lecteurs qui ne considèrent que le côté pratique des choses, il en est deux sur-tout qui ont dû les frapper :

J'ai, par exemple, admis la possibilité de distinguer dans la description spéciale et dans le parcellaire qui l'accompágne, les peuplements dont les âges ne 
diffèrent que d'un an. J'ai admis, en outre, que le parcellaire pouvait être assez détaillé pour que chaque coupe contînt plusieurs parcelles.

Ces suppositions, évidemment forcées ${ }^{1}$, avaient - pour but de faciliter mes démonstrations, de rendre plus intelligible, en le complétant, le mécanisme de la formation du plan d'exploitation. Pour faire comprendre ce mécanisme, il ne fallait pas seulement le réduire à la plus simple expression, en l'étudiant dans les taillis; il fallait supposer toutes les conditions propres à mettre en évidence jusqu'aux plus petits de ses rouages.

En théorie, on est absolu, on ne recule devant aucune hypothèse, lorsqu'elle est utile à une démonstration, et qu'elle n'est pas d'ailleurs manifestement contredite par la nature des choses.

En pratique, on fait ce que l'on peut, dans l'ordre des choses réellement utiles; mais pour ne point se tromper dans le choix des choses possibles et ntiles, il est indispensable d'être pénétré de tout ce que comporterait l'application rigoureuse de la thóorie.

En réalité, on ne s'attache pas, je l'ai déjà dit, dans la division d'une forêt en parcelles, à ces nuances à peine perceptibles qui résulteraient de l'accroissement d'une année en plus ou en moins. En réalité, on fait ordinairement ces parcelles assez. grandes pour que l'on puisse en former plusieurs

1. Voir la note page 180. 
coupes; on néglige, enfin, beaucoup de détails auxquels je me suis cependant arrêté. Ai-je eu tort d'agir ainsi? - Je ne le pense pas.

Il serait peu rationnel de voir la preuve d'un esprit minutieux dans les soins apportés à l'étude d'une question. Tous les préceptes petits ou grands que l'on déduit de cetle étude, apprennent, en supposant qu'ils ne trouvent pas leur application sur. le terrain, à observer avec méthode et avec fruit, et à ne négliger dans ses investigations aucun point essentiel. - C'est un précieux avantage.

Ce n'est pas dans la théorie que l'on doit craindre les délails; ils ne sont jamais inutiles quand ils sont mis à la place convenable, et ils sont même nécessaires pour l'enchaînement logique des idées, la - force du raisonnement. C'est dans la pratique seulement que les détails peuvent être regrettables; mais je crois que c'est précisément quand on s'est appliqué à ne pas les négliger dans le premier cas, que l'on s'en affranchit avec le plus d'à-propos dans le second. 


\section{CHAPITRE TROISIÈME.}

\section{DU PLAN D'EXPLOITATION DANS LES FUTAIES}

\section{TRAITÉES}

PAR LA MÉTHODE DU RÉENSEMENCEMENT NATUREL ET DES ÉCLAIRCIES PÉRIODIQUES.

En continuant mon élude sur la formation du plan d'exploitation dans les aménagements de forêts, je crois devoir prévenir que l'on tirerait peu de profit de ce que j'ai à dire sur les futaies, si l'on n'avait pas présents à la mémoire les principes et les considérations que j'ai développés, en traitant la même question pour les taillis. Je serai en effet obligé, d'abord, pour éviter des redites fastidieuses, et ensuite pour mettre en relief les points essentiels et fondamentaux de l'opération qui va faire l'objet de ce chapitre, de sous-entendre beaucoup de points secondaires et beaucoup de détails qui ont été discutés dans le précédent.

Qu'on ne s'étonnne pas de cel avertissement; si je me donne la peine d'écrire, ce n'est pas précisément pour les gens instruits, c'est pour ceux qui seraient embarrassés de donner une définition exacte et complète des mots série, affectation, classe d'âge, parcelles, etc., et qui procèdent à un martelage sans 
se soucier d'autre chose que de ce qui se trouve dans l'enceinte de la coupe; sans songer qu'entre cette coupe et le surplus de la forêt, il existe une solidarité dont ils devraient tenir compte; sans se préoccuper enfin le moins du monde de subordonner leurs opérations à un plan d'ensemble, seul moyen pourtant de les faire concourir à un même but.

Ces erreurs, profondément regrettables, seraient évidemment moins communes, si la notion du plan d'exploitation était plus répandue.

\title{
ARTICLE' PREMIER.
}

\author{
DES RAISOXS CULTURALES QCI, S'OPPOSEXT \\ $A$ CE QUE L'ON ADOPTE, \\ POC LE TABLAD DES EXPLOITATIONS DES FUTAIES, \\ LB MEAE CADRE QUE POURTELUI DES TAILLIS.
}

Nous avons à régler la marche et lá quotité des exploitations d'une futaie, dont la contenance est de 200 hectares, dont les parcelles sont toutes exploitables à 100 ans $^{1}$; nous avons sous les yeux le cahier de la description spéciale, et le plan topographique sur lequel chaque parcelle figure avec l'indication de son étendue et de son âge; essayerons-nous, et suffi-

4. Ce chiffre de 100 ans n'a aucune signification pratique. Il est évidemment trop faible pour la plupart des futaies, et on verra plus tard qu'il convient d'ailleurs d'adopter autant que possible pour la révolution un chiffre divisible par un grand nombre de facteurs. (Nole de la 2 e édition.) - 
rait-il, pour atteindre le but que nous nous proposons, de remplir un tableau semblable à celui que nous avons dressé pour le taillis simple et qui comprendrait, en conséquence, autant de colonnes qu'il y a d'années dans l'âge d'exploitabilité, ou, ce qui revient au même, dans la révolution?

Un semblable travail rencontrerait des difficultés mécaniques presque insurmontables, dans le grand nombre des colonnes entre lesquelles devrait se faire la répartition des parcelles; mais il y a pour ne pas l'entreprendre une raison culturale péremptoire, raison qui consiste en ce que l'ápplication de la méthode du réensemencement naturel et des éclaircies périodiques ne pourrait se concilier avec un plan dans lequel la contenance, l'emplacement et l'époque des coupes annuelles, seraient préalablement et invariablement fixés.

Admettons en effet que surmontant les difficultés mécaniques de la répartition des parcelles, on soit parvenu à classer ces dernières dans les cent colonnes du tableau d'exploitation; supposons que, la gradation des âges étant parfaitement régulière et les conditions de végétation uniformes, on ait trouvé une égale contenance pour le total de chaque colonne; faisons abstraction des règles d'assiette.

Nous serait-il permis d'affirmer, en présence de cet état de choses, que le plan d'exploitation ne laisse rien à désirer, et qu'il ne reste plus qu'à l'appliquer sur le terrain?

Nous n'y serions nullement autorisés, et c'est ce 
dont on s'assurera facilement, si on se rappelle, d'une part, le troisième des principes que nous avons posés comme l'une des bases essentielles d'un bon plan d'exploitation, et, de l'autre, les règles de culture relatives au traitement des futaies destinées à être régénérées par les semis naturels.

Que dit ce principe? - Que l'on doit établir pour la répartition des produits à réaliser, dans le cours de la révolution, un règlement qui, tout en se conciliant avec les exigences de la culture, soit de nature à assurer le rapport annuel soutenu.

Que disent ces règles? - Que l'exploitation des massifs à régénérer doit être faite en plusieurs fois, si l'on veut procurer d'abord l'ensemencement complet du terrain, placer les graines dans les conditions les plus favorables à leur germination, et ne livrer ensuite les jeunes repeuplements aux influences de l'atmosphère, qu'avec les ménagements que réclane leur tempérament.

On ne saurait donc prévoir le nombre d'arbres que l'on abattra dans la coupe d'ensemencement; on ne saurail préciser ni l'époque, ni l'importance, ni l'emplacement de la coupe secondaire et de la coupe définitive; on ne saurait enfin fixer le rang de chacune des coupes de régénération, lui donner un numéro d'ordre et l'exploiter dans l'année correspondante; et, par conséquent, un plan dans lequel ces différents points seraient arrêtés, violerait les règles élémentaires de la culture. Nous verrons plus tard que tous les efforts faits pour concilier ces règles 
avec le rapport soutenu et les avantages inhérents à la détermination, par contenance, des coupes annuelles, n'ont abouti et ne pouvaient aboutir à aucun résultat pratique. Pour le moment, je me borne à constater qu'il n'est pas possible de régler d'avance dans une futaie, comme on a proposé de le faire dans les taillis, l'ordre dans lequel se succéderont les coupes principales annuelles.

Ainsi, pas de doute à cet égard : le cadre adopté pour la formation du tableau d'exploitation des taillis, est inadmissible pour la formation du même tableau, dans les futaies traitées par la méthode du réensemencement naturel et des éclaircies périodiques. Il est inconciliable avec les principes de la culture, surtout quand on les considère dans leur application aux coupes principales.

\section{ARTIGLE II.}

DE LA DIVISION DE LA RÉ VOLUTION PRINCIPALE EN PÉRIODES ET DU PARTAGE DE LA FORÊT

EN AFFECTATIONS CORREPONDANTES.

Le cadre dont nous nous sommes servi pour dresser le tableau des exploitations du taillis simple offre cependant de grands avantages, au triple point de vue de la simplicité, de la facilité et de la sûreté qui en résultent pour la marche de ces exploitations. Il est désirable, en conséquence, que l'on s'en écarte le moins possible dans la formation du tableau 
d'exploitation de la futaie, et que l'on y apporte seulement les modifications impérieusement commandées par l'intérêt de la conservation et de la régénćration des massifs.

Si l'on ne peut prévoir l'année dans laquelle devra se faire l'une quelconque des coupes de régénération, sur un point déterminé, on peut, sans se tromper, assurer que cette coupe aura lieu dans une certaine période de temps.

Étant donné un massif exploitable en vingt années, si on ne peut, sous peine de compromettre sa régénération, régler la contenance des coupes annuelles et fixer, en outre, l'ordre dans lequel ces coupes devront se succéder, on comprend qu'en laissant à un forestier expérimenté la faculté d'exploiter ledit massif de la manière qu'il jugera la plus. convenable, il se charge de le régénérer complétement dans le délai voulu.

Ceci admis, il s'ensuit que s'il n'est pas possible d'arrêter, dans le plan d'exploitation d'une forêt divisée en cent parties, exploitables en cent ans. l'ordre dans lequel ces cent parties devront être successivement exploitées et régénérées, il est permis de demander et de prescrire que l'on coupe et que l'on régénère, dans un certain nombre d'années, préalablement fixé et obligatoire, un même nombre de parties prises dans leur ensemble.

Quel sera ce nombre d'années, quelle sera la durée de la période pendant laquelle l'assiette des coupes devra rester indéterminée? 
Il est aisé de le deviner:

Cette durée sera all moins égale au double de l'intervalle probable de temps qui séparera, pour un même point, la coupe d'ensemencement de la coupe définitive. Si cet interva!le est de $\mathbf{1 0}$ ans, la durée de la période devra être au moins de 20 ans; s'il est de 5 ans, la période pourra être réduite à 10 ans; s'il était égal à zéro, ce qui signifierait que, par une seule et même coupe, on pourrait enlever tous les arbres exploitables et assurer le repeuplement, la période deviendrait nulle, et la fixalion par contenance des coupes annuelles, possible.

Plaçons-nous dans la première hypothèse d'un intervalle de 10 ans, entre la coupe d'ensemencement et la coupe définilive. Supposons aussi, pour ne pas compliquer inutilement.la démonstration, que les coupes claires ou secondaires ne soient pas nécessaires, et que les arbres exploitables dans une contenance déterminée se distribuent par portions égales, entre la coupe d'ensemencement et la coupe définitive. Mettons-nous, enlìn, en présence de la forêt que nous avons déjà prise pour exemple, laquelle serait une futaie partagée en cent parties, exploitables en cent ans. Cette année, nous nous transporterons dans la partie la plus âgée, et nous y procéderons à une coupe d'ensemencement; mais cette coupe ne devant nous donner que la moitié des arbres que nous aurions à prendre, si nous n'avions à nous préoccuper de la régénération, nous comblerons le déficit en pratiquant une autre coupe d'ense- 
mencement sur toute la partie contiguë. L'année prochaine, nous ferons, par des raisons analogues, les premières coupes de régénération sur la troịsième et la quatrième partie, 'et dans dix ans, c'est-à-dire à l'époque où nous pourrons revenir au pointde départ pour commencer les coupes définitives, nous aurons les vingts parties les plus âgées de la forêt, à l'état de coupes d'ensemencement. Or, il est clair que celles de ces vingt parties qui auront été mises les dernières en cet état, ne pourront être exploitées définitivement que 10 ans plus tard, c'est-à-dire dans 20 ans.

La durée de da période pendant laquelle l'assiette et la contenance des coupes resteront incertaines, dépend donc de la promptitude plus ou moins grande avec laquelle les peuplements sont susceptibles de se régénérer par les semences. Toutes les essences ne présentent pas, sous ce rapport, les mêmes propriétés. La nature du climal et la qualité du sol.influent d'ailleurs beaucoup sur les repeuplements naturels. Toutefois, on peut porter en général à une douzaine d'années, en moyenne, le délai nécessaire pour la régénération suffisamment complète d'un peuplement quelconque, si l'on a soin, d'ailleurs, de venir en aide à la nature par des travaux intelligents. Les forestiers qui ne parviennent pas à obtenir des repeuplements naturels, qui sont disposés à les croire impossibles, et qui en accusent la force des choses, devraient en accuser surtout leur incurie. II est probable qu'ils changeraient d'avis s'ils prenaient quelques soins pour faciliter ces repeuplements par 
des préparations de terrain faites en temps opportun, etc. Au surplus, si, malgré toutes les précautions prises, il devait s'écouler plus de 10 à 12 . ans entre la coupe d'ensemencement et la coupe définitive, je conseillerais de recourir alors aux repeuplements artificiels, afin de ne pas être amené à prolonger la durée de la période d'exploitation. En effet, il ne faut pas perdre de vue que tout retard dans la régénération d'un peuplement exploitable se traduit par une diminution dans le revenu, et qu'on méconnaîtrait les règles d'une saine économie, si on permettait que le chiffre de cette diminution pût s'élever au-dessus des frais qu'occasionnerait le semis ou la plantation du terrain à régénęerrer ${ }^{1}$.

Une autre considération pour ne pas prolonger au delà de 20 à 24 ans la durée de la période dont nous nous occupons, c'est qu'il convient de restreindre, autant que possible, la faculté dẹ comprendre dans les exploitations, des bois qui ne sont pas encore parvenus à l'âge d'exploitabilité.

A ces principes ajoutons-en un autre : il importe beaucoup que la période en question contienne un nombre d'années qui soit une partie aliquote de la révolution. S'il n'en était pas ainsi, on șe verrait obligé d'adopter pour les périodes successives de cette révolution une durée inégale, èt on s'exposerait dès lors, soit à rester en deçà, soit à aller au delà du

1. Sur la durée de la période, voir la note au bas de la page 280. 
terme reconnu le plus convenable, eu égard aux exigences de la culture ${ }^{1}$.

Ces préliminaires posés, mes lecteurs doivent maintenant prévoir quelles sont les modifications que nous ferons subir au tableau d'exploitation du taillis, pour l'approprier à la futaie. Au lieu de dresser un cadre comprenant autant de colonnes qu'il y a d'années dans la révolution, et précisant la partie de la forêt, la parcelle ou portion de parcelle à régénérer chaque année, nous nous bornerons à ouvrir autant de colonnes qu'il y a de périodes dans la révolution, et à préciser la partie de forêt, les parcelles et portions de parcelles, à régénérer dans le cours de chaque période. Le plan d'exploitation ne fixera plus l'assiette et la contenance de chaque coupe principale annuelle; il fixera seulement l'assiette et la contenance des coupes principales, considérées en bloc, à faire dans chacune des périodes de la révolution. La révolution principale étant, par exemple, de 100 ans, et le temps reconnu néceśsaire pour régénérer naturellement le peuplement étant de 10 ans, il ne s'agit plus de former un plán qui fournisse, chaque année, une coupe dont le produit soit constant; il s'agit d'en former un qui partage la forèt en cinq parties, exploitables chacune dans. une période de 20 ans, et qui soient telles qu'on

1. Quand nous aurons à traiter des conversions, on verra qu'il est utile en outre que la durée de la période soit divisible par le nombre des périodes, et en général par le plus grand nombre de facteurs possible. (Note de la $2^{\mathrm{e}}$ édition.) 
en obtienne autant que possible des produits égaux.

Ces parties de forêt à régénérer successivement dans les périodes d'une révolution principale ont reçu le nom d'affectations. On les distingue l'une de l'autre par un numéro d'ordre différent, selon qu'elles correspondent à la première, à la deuxième, etc., ou à la dernière période, et on donne aux parcelles une désignation définitive qui permet, au moyen d'un indice numérique, de reconnaître à quelle affectation elles appartiennent ${ }^{1}$.

1. Quand les idées que je viens d'exposer sur la formation des affectations ont été publièes pour la première fois dans les Annales forestières, elles ont soulevé des objections. Voici ces objections et la réponse que j'y ai faite. On verra que je suis d'accord avec mon contradicteur sur l'influence que peuvent avoir les coupes secondaires en ce qui concerne la durée de la période; mais je ne peux rien concéder sur les autres points.

\section{OBJECTIONS.}

* Monsieur le rédacteur,

"Dans votre article sur la formation du plan d'exploitation, vous dites que la durée de la période doit etre au moins égale au double de l'intervalle qui séparera la coupe d'ensemencement de la définitive; si' cet intervalle est de dix ans, la durée de la période devra être au moins de vingt ans. - Pour le démontrer, vous vous placez dans cette hypothèse que les coupes claires n'étant pas nécessaires, les arbres exploitables dans une contenance déterminée se distribueront par portions égales entre la coupe d'ensemencement et la coupe définitive. Partant de là, vous arrivez très-logiquement aux conséquences annoncées.

"Mais si vous vous étiez mis dans une hypothèse plus conforme à la généralité des circonstances, en admettant, par exemple, que la coupe claire doit être faite cinq ans après la coupe sombre, la coupe défnitive cinq ans après la coupe claire, et que les bois 


\section{Je viens de démontrer la nécessitẻ de ne pas se} limiter dans l'année, pour l'assiette des coupes prin-

exploitables se distribuent par portions égales et par liers entre les trois coupes de régénération, la conclusion eủt été bien différente; car on peut dire, en suivant votre méthode de démonstration et en se meltant en présence d'une futaie parlagée en cent parties exploitables en cent ans, savoir :

a La première année, nous nous transporteróns dans la partie la plus àgée, et nous y ferons coupe sombre; mais cette coupe ne devant nous donner que le tiers du matériel sur pied, nous comblerons le déficit en pratiquant deux autres coupes d'ensemencement sur toute l'étendue des deux autres parties successivement contiguës. L'annee suivante, nous ferons, par des raisons analogues, les premières coupes de régénération sur les quatrième. cinquième et sixième parties, et dans cinq ans, c'est-à-dire à l'époque où nous pourrons revenír au point de départ, pour commencer les coupes secondaires, nous aurons les quinze parties les plus ågées de la forêt à l'état de coupes d'ensemencement; cinq ans plus tard, c'est-à-dire dix ans après l'ouverture de la période, ces mèmes quinze parties seront à l'état de coupes secondaires. Or, il est clair que celles de ces quinze parties qui auront été mises les dernières dans cet état ne pourront ètre exploitées définitivemẻnt que cinq ans plus tard, c'est-à-dire dans quinze ans.

* Au résumé, dans la plupart des cas, pour'les futaies de sapin et de hêtre, ne serait-on pas fondé à dire que la durée de la période doit étre au moins égale au triple de l'intervalle supposé constant qui sépare, soit la coupe d'ensemencement de la coupe secondaire, soit la coupe secondaire de la coupe définitive, ou, en d'autres termes, égale à l'intervalle qui s'écoule entre la coupe sombre et la coupe définitive, augmenté de sa moitié?

a La durée des périodes, el par conséquent celle des révolutions, serait donc ainsi un multiple de trois, dans tous les cas où il serait nécessaire de passer par trois coupes, pour consommer la régérération des massifs. Mais d'autres considérations plus importantes devant présider au choix du terme d'exploitabilite, ne sera-t-on pas très-souvent forcé de négliger celte con- 
cipales, dont le plan d'exploitation est destiné à régler la marche. La même nécessité existe souvent

dition, à laquelle vous voulez subordonner la durée des périodes?

"Voilà ma première objection; je vous demande aussi une explication à propos du deuxième paragraphe de la page 283. "Une autre considération, dites-vous, pour ne pas prolonger au " delà de vingt ans la durée de la période, c'est qu'il convient de " restreindre, autant que possible, la faculté de comprendre dans "les exploitations des bois qui ne sont pas encore parvenus à "l'âge d'exploitabilité. "

“ Si je comprends bien, vous entendez par là prendre des garanties contre la nẹgligence ou la légèreté des agents chargés d'exécuter un aménagement, et suspecis d'entamer d'abord les massifs les moins âgés. de l'affectation. Mais n'a-t-on pas, pour les maintenir dans la bonne voie, un plan d'exploitation spécial, indiquant l'ordre dans lequel les parcelles qui composent cette affectation devront venir successivement en tour d'exploitation? Quant à ceux qui prendraient à tâche d'enfreindre les dispositions de l'aménagement, les courtes périodes ne les arrêteront pas. Il semble, au contraire, que plus la période sera courte, plus souvent on se trouvera dans la nécessité d'entamer, avant son expiration, les massifs de l'affectation contiguë, par suite de l'insuccès des coupes d'ensemencement ou des coupes secondaires pratiquées dans l'affectation de la première période, et, s'il faut ainsi anticiper, voilà nos agents pleinement investis de la faculté dont vous vouliez les priver.

. “Jusqu’à preuve contraire, je ne reconnais donc à la courte durée des périodes qu'un seul arantage, celui de faciliter la recherche de la possibilité, en diminuant les chances d'erreur inhérentes aux calculs d'accroissement. Yous posez ensuite en principe que la durée de la période doit être une partie aliquote du chiffre de la révolution, sous peine de s'exposer soit à rester en deçà, soit à aller au delà du terme reconnu le plús courenable, eu égard aux exigences de la régénération.

"Cette conséquence me parastt très-contestable, et je n'attribue à l'égalité des périodes qu'un mérite de symétrie; je vais plus loin : il serait plus commode, à mon gré, d'admettre en 
pour les coupes intermédiaires, car elles sont plus ou moins impérieusement subordonnées aux autres.

principe l'inégalité des périodes, parce qu'il est rare que les párcelles, telles qu'on les a tout d'abord établies sur le terrain, puissent être exactement colloquées dans des périodes égales.

" De là résulte que, pour égaliser les affectations périodiques, il faut après coup venir scinder les parcelles au moyen de lignes de contenance qui compliquent inutilement le parcellaire et surchargent les plans. Ne serait-il pas plus simple de proportionner la durée des périodes à la contenance des parcelles ou des groupes de parcelles?

a Par exemple, étant donnée une forêt de 100 hectares, aménagée à cent ans, et partagée sur le terrain d'après les considérations de situation, de consistance et de fertilité, en cinq parcelles dont les contenances seraient de $40,12,15,18$ et 15 hectares; quel inconvénient, je le demande, y aurait-il à affecter chacune de ces parcelles à une période dont la durée serait égale au nombre d'hectares qui y seraient colloqués, la révolution se trourant ainsi partagée en cinq périodes de quarante, douze, quinze, etc., annẻes? Cela scrait assurément plus simple. La régénération de chaque affectation périodique ne pourrait peut-être pas s'effectuer exactement durant la période correspondante, j'en conviens, et l'on serait obligé, avant l'expiration d'une quelconque des périodes, d'entamer les coupes dans l'affectation immédiatement conliguë; mais c'est ce qui arrive et ce qui arrivera toujours plus ou moins, quelle que soit la durée des périodes, et ces emprunts 'peuvent s'opérer, je crois, sans porter aucune atteinte à l'exploitabilité adoptée. Đ

\section{RÉPONSE.}

On nous reproche, d'abord, d'avoir négligé de faire entrer les coupes claires dans l'hypothèse que nous avons admise, afin de monitrer comment on pouvait arriver à apprécier la durée de la période et l'étendue de l'affectation correspondante.

On nous prouve en même temps qu'en tenant cọpte de la coupe claire, et en supposant qu'elle ait lieu précisément au milieu de l'intervalle de temps qui sépare la coupe d'ensemence- 
On ne pourrait, par exemple, affirmer que, telle année, telle parcelle aura besoin d'être éclaircie,

ment de la coupe définitive, la durée de la période nécessaire pour compléter la régénération de l'affectation correspondante, devient égale à cet intervalle multiplié par 1,0 .

Cette dernière proposition est parfaitement exacte, lorsque la coupe claire égale en importance la coupe d'ensemencement ou la coupe définitive, et qu'elle intervient en outre précisément au milieu de l'intervalle de temps qui les sépare.

Mais si la coupe claire est plus rapprochée de la coupe définitivelque de la coupe d'ensemencement, si elle est moius productive, si elle se fait en plusieurs fois, dans cliacun do ces cas elle peut et doit méme avoir pour effet de retarder le délai indispensable pour compléter la régénération des coupes formant l'étendue de l'affectation.

En conséquence, si l'on ne considère que le nombre des exploitations à faire sur un point donné, pour en effecluer le repeuplement naturel, on a raison de regarder notre démonstration comme incomplète; mais si l'on nous accorde que la coupe d'ensemencement et la coupe défnitive sont celles 'dont l'importance et l'écart sont le plus faciles à apprécier, et exercent, en outre, le pllus d'influence sur le temps que réclame la régénélation complète d'un certain nombre de coupes envisagées dans leur ensemble, on s'expliquera que, pour ne pas compliquer notre démonstration, nous l'ayons dégagée des éléments secondaires et variables qui préoccupent notre correspondant.

- Répondons aux autres critiques :

Lorsque nous avons dit qu'il fallait restreindre autant que possible la durée de la période, afin de ne pas être exposé à exploiter des bois trop éloignés d'avoir atteint l'âge d'exploitabilité, nous sommes partis de celte supposition que les agents clrargés de l'exécution de l'aménagement auraient le droit et pourraient mème être forcés de se mouroir, pour l'assietle des coupes annuelles, dans toute l'étendue de l'affectation périodique.

Si l'on admettait, au contraire, comme semble le vouloir notre correspondant, que l'on pût assujettir cette assiette à une marche précise, au moyen d'un plan spécial d'exploittion, les périodes 
puisqu'on ne sait pas à quelle époque elle sera régénérée; mais on peut affirmer qu'elle aura' besoin de

n'auraient plus de raison d'être; car, encore une fois, elles ne sont motivées que par l'npossibilité de concilier l'assiette fixe des coupes annuelles avec les exigences de la régénération.

On voit donc que le seul avantage des périodes ne consiste pas à faciliter la recherche de la possibilité, en diminuant les chances d'erreur inhérentes aux calculs d'accroissement. Si c'étail à leur seul avantage, comme il serait d'autant plus grand quel les périodes seraient plus courtes, on derrait logiquement ne leur donner qu'un an de durée.

Mais, cela posé, il est évident que si l'on veut renfermer la latitude laissée aux agents d'exécution, pour l'assielte des coupes, dans les limiles strictement conrenables, il faut que les périodes de la rérolution soient égales entre elles, car, sans cela, il y en anrait qui seraient nécessairement ou trop longues ou trop courtes.

Supposons, avec notre contradicteur, qu'une forèt ait été partagée en cinq parlies, savoir : la première de $\mathbf{4 0}$ hectares, la deusième de 12 , la troisième de 40 , la quatrième de 18 , la cinquième de 15 , et que chacune d'elles ait été affectée à une période d'une durée égale au nombre d'hectares qu'elle contient: n'y aurait-il pas de grands inconvénients à laisser pendant quarante ans, et pour une affectation qui comprendrait presque moitié de la forét, l'assiette des coupes annu elles subordonnée à l'appréciation, au bon plaisir, à l'arbitraire des agents d'exécution; et, s'il n'est pas possible de fixer l'imp ortance, l'emplacement et l'ordre des quarante coupes à faire dans celte affectation, ne pourrait-on déterminer ces différents points pour un nombre de coupes, prises en bloc, inférieur à quarante? L'affirmative n'est pas contestable, et comme il convient que tout ce qui peut ètre fixé le soit, et qu'on ne s'écarte - de la possibilité par contenance que dans la mesure que comportent les exigences de la culture, il est certain que l'on ferait une grande faute si l'on ne diminuait pas la durée de la première période.

Mais si cette période doit étre considérée comme trop longue, celle de douze ans sera probablement trop courte, en ce sens 
l'être dans la période qui suivra celle de"sa régénération; en 'sorte que, lorsque les affectations sont constituées, lorsqu'on est fixé sur la période dans laquelle une parcelle devra être obligatoirement régénérée, il devient aisé de se fixer également sur les périodes dans lesquelles elle devra être ultérieurement éclaircie.

On voit combien la division de la révolution en périodes et le partage de la forêt en affectations correspondantes, sont de nature à faciliter les opérations culturales à faire dans la forêt que l'on se propose d'aménager. Ce règlement n'est, toutefois, qu'un acheminement au but de l'aménagement; il ne saurait, quelque bien établi qu'il futt d'ailleurs, constituer à lui seul le plan d'exploitation; il n'en est que la charpente, le canevas; il remplit dans l'aménagement un rôle analogue à celui de la triangulation dans la géodésie, et de même que la triangulation est faite pour faciliter les levés de détail, de même

qu'elle ne permettra pas, quelle que soit d'ailleurs l'habileté qu'on y apporte, de régénérer dans un aussi court délai les 12 hectares composant l'affectation, et que, bien avant d'arriver à l'expiration de la période, on se verra dans la nécessité d'anticiper sur l'affectation voisine.

On nous dit, il est vrai, que quelle que soit la durée de la période, il est impossible d'éviter que l'on entame avant son expiration l'affectation contiguë. Nous n'admettons pas d'une manière absolue cette impossibilité. Sans doute, les anticipations dont il est question sont difficiles à éviter ; mais c'est une raison de plus pour ne négliger aucune des précautions nécessaires afin d'en diminuer au moins les inconvénients.

Si l'on abandonne ce principe, on tombe dans l'empirisme. 
le règlement des exploitations par période est fait pour faciliter celui des exploitations annuelles; mais il n'y supplée pas. La division de la révolution en périodes, et le partage de la forêt en affectations correspondantes, permettent, comme je le montrerai plus clairement par la suite, de réaliser le rapport soutenu par période; c'est beaucoup, sans doute, mais ce qu'on attend surtout de l'aménagement, c'est la réalisation du rapport soutenu par année, et, pour cela, il faut trouver les moyens de répartir d'une manière égale le produit d'une affectation, entre les années de la période correspondante. Ces moyens comportent un règlement qui, joint au premier, complète le plan d'exploitation.

Ainsi, dans la présente étude sur le plan d'exploitation des futaies, j’aurai à traiter successivement :

$1^{\circ}$ De la formation des affectations;

$2^{\circ}$ Du règlement général des exploitations par période;

$3^{\circ}$ Du règlement des exploitations annuelles.

Mais dans les futaies, plus souvent encore que dans les taillis, l'irrégularité des peuplements conduit à adopter, tantôt une marche provisoire pour les coupes, tantôt une révolution principale transitoire ou une révolution préparatoire; et ces nécessités donnent lieu à des complications dont je sortirais difficilement, si je ne faisais de chacun des cas où elles se présentent, l'objet d'un chapitre spécial.

J'appliquerai donc mon étude à trois hypothèses: 
$1^{\circ}$ Celle d'une révolution principale définitive, et d'une marche des coupes, normale;

20. Celle d'une révolution principale définitive, et d'une marche des coupes, provisoire;

$3^{\circ}$ Celle d'une révolution principale transitoire oủ d'une révolution préparatoire, et d'une marche des coupes, provisoire. 


\section{CHAPITRE QUATRIEME.}

DU PLAN D'EXPLOITATION daNs LES FUtAiEs, LA REVOLUTION PRINCIPALE POUVANT ETtRE DEFINTIVE.

ET LA MARCHE DES COUPES NORMALE.

\section{ARTICLE PREMIER. • \\ FORYATION DES AFFECTATIONS.}

\section{$\$ 1^{\text {er. }}$}

Tableau des affectations.

Nous avons affaire à une forêt de 200 hectares, exploitable dans une révolution de cent ans. Cette révolution a été divisée en cinq périodes égales. II s'agit de partager la forêt en un même nombre d'affectations correspondantes. Nous remplirons le tableau ci-contre.

J'ai maintenu la première partie du tableau d'exploitation des taillis, partie destinée à recevoir le résumé de la description spéciale. On en comprend l'utilité. Il est important que le tableau des affectations 


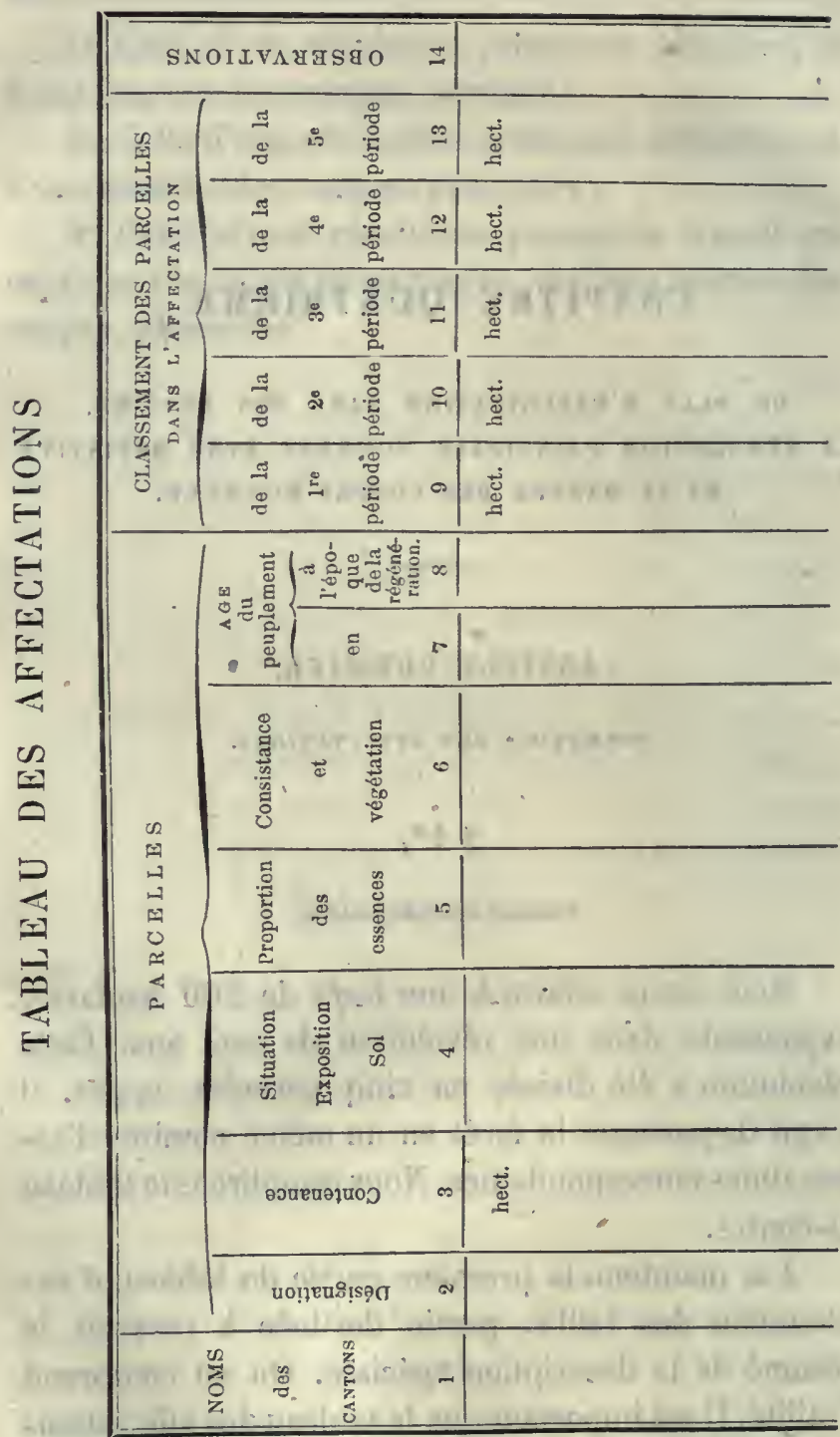


renferme les indications sommaires, qui sont de nature à justifier l'ordre adopté pour la succession des coupes; car ce tableau est, comme je l'ai déjà dit, le document essentiel et fondamental de l'aménagement, et il doit être établi de telle sorte, qu'on puisse à la rigueur juger de son mérite, sans recourir à d'autres éléments d'appréciation que ceux qui y sont contenus.

J'ai substitué à la seconde partie du tableau, destinée à présenter l'ordre des. exploitations, cinq colonnes dont l'objet est clairement indiqué par leur en-tête. On peut regarder chacune d'elles comme un compte ouvert à l'affectation dont elle porte le numéro. Toutes les parcelles qui devront être régénérées dans la première période seront inscrites dans la première colnnne, et composeront la première affectation. Toutes celles qui devront être régénérées dans la deuxième période seront inscrites dans la deuxième colonne, et composeront la deuxième affectation, etc., etc.

Mais sur quelles considérations se fondera-t-on pour déciden que telle parcelle doit être régénérée dans telle ou telle période?

On se fondera sur les considérations que nous avons fait intervenir dans la discussion du plan d'exploitation des taillis.

On fera donc le classement des parcelles dans les affectations, de manière à rendre possibles des exploitations qui satisfassent tout à la fois, dans la mesure que comporte la nature des choses, aux exigences de 
l'exploitabilité, aux règles sur l'assiette des coupes, et au rapport annuel soutenu.

Appliquons rapidement à chacun de ces côtés de la question, les préceptes que j'ai donnés, en traitant de l'aménagement des taillis.

\section{$\S 2$.}

Formation des affectations suivant l'âge d'exploitabilité.

Si toutes les parcelles renfermaient des peuplements susceptibles de rester sur pied jusqu'au terme fixé par la révolution, leur classement dans les affectations ne rencontrerait aucune difficulté.

La parcelle $\mathbf{A}$ renferme un peuplement âgé moyennement de 35 ans; elle ne sera exploitable que dans 65 ans; on la classera dans l'affectation de la quatrième période.

- La parcelle B renferme ún massif de vieux arbres clair-plantés, surmontant un jeunè répeuplement; l'exploitation de ces vieux arbres sous forme de coupe secondaire ou définitive est urgente; on les classera dans l'affectation de la première période.

Rien de plus simple.

Mais il y a souvent des parcelles dont le peuplement n'est pas susceptible de végéter jusqu'au terme fixé par la révolution, et qu'il faut, en conséquence, exploiter avant ce terme.

Cette nécessité peut tenir à une circonstance accidentelle : il s'agit d'un peuplement qui, quoique 
jeune encore, ne jouit déjà plus d'une vigoureuse végétation, soit qu'il ait été abrouti dans sa jeunesse, soit qu'il provienne de vieilles souches, soit que des éclaircies maladroites aient ébranlé sa constitution.

Dans ces divers cas, l'affectation à laquelle une parcelle appartient, n'est pas indiquée par la différence existant entre l'âge normal d'exploitabilité et l'âge de cette parcelle, mais par le temps, difficile à apprécier d'ailleurs, pendant lequel le peuplement de la parcelle en question pourra rester sur pied sans dépérir.

La nécessité d'exploiter une parcelle avant le terme fixé par la révolution peut dépendre d'une circonstance permanente :

Cette parcelle se compose d'une essence dont la longévité est moins grande que celle d'après laquelle a été fixée la durée de la révolution. Ici, de deux choses l'une : ou l'âge d'exploitabilité de cette essence est une parlie aliquote de la révolution, ou il ne l'est pas. Dans le premier cas, on fait figurer la parcelle dans autant d'affectations différentes que son âge est renfermé de fois dans la révolution; dans le second cas, on la laisse en dehors de l'aménagement, à moins qu'on ne juge convenable d'y pratiquer une substitution d'essences, qui permette de concilier son exploitation avec celle du surplus de la forêt, et alor's son classement rentre dans les conditions déjà prévues.

Nous avons à nous occuper d'une parcelle de pins 
ou de bouleaux exploitables à cinquante ans et âgés aujourd'hui de vingt-cinq ans; nous la classerons dans l'affectation de la deuxième période et dans celle de la quatrième.

On pourrait prévoir des circonstances où une parcelle devrait figurer dans toutes les affectations. C'est ce qui aurait lieu s'il s'agissait de comprendre dans l'aménagement de notre forêt, exploitable à cent ans, une parcelle exploitable en taillis à l'àge de vingt ans.

Mais ce sont là des hypothèses qui ont peu d'utilité pratique, et que je fais seulement pour ne pas laisser ma démonstration incomplète.

Dans la pratique, on.évite généralement de comprendre dans le même aménagement, des peuplements exploitables à des âges différents; car en compliquant le plan d'exploitation, ils deviennent en même temps une cause de confusion et de difficultés, et aucun motif sérieux ne commande de se résigner à de tels inconvénients.

Ils deviennent une cause de confusion et de difficultés; parce qu'ils s'opposent à l'application des règles d'assiette; parce qu'ils contrarient la réalisation du rapport soutenu; parce qu'ils obligent de ramener les exploitations sur un point donné, plus souvent que sur les points environnants.

Aucun motif sérieux ne commande de se résigner à de tels inconvénients. Le plus important de tous les intérêts, celui de la consommation, ne saurait par exemple y rien gagner : en effet, lorsque le con- 
sonmateur sollicite un produit d'une nature particulière, ce n'est pas d'une manière intermittente, c'est d'une nanière 'permanente; ce n'est pas tous les cinquante ans, tous les vingt ans, c'est chaque année. Si donc les parcelles de pins, de bouleaux ou de taillis, dont je parlais tout à l'lieure, étaient assez grandes pour fournir chaque année une conpe, il faudrait en former une série particulière; dans le cas contraire, il conviendrait de les régénérer en essences susceptibles de parcourir la révolution applicable à la masse, et, si celte régénération était inpossible, je serais d'avis de les laisser en dehors de l'aménagement.

\section{$\$ 3$.}

Formation des affectations conformément aux règles d'assiette.

Cette formation doit être examinée à deux points de vue :

$1^{\circ}$ Au point de vue de la marche des coupes dans chaque affectation;

$2^{\circ}$ Au point de vue de la position respective que les affectations doivent occuper sur le terrain.

Pour que la marche des coupes, dans chaque. affectation, puisse se conformer aux règles d'assiette, il est bon que les affectations aient une forme régulière; qu'elles présentent leur moindre largeur aux vents les plus violents; qu'elles soient traversées et limitées par des chemins, mais, par-dessus 
tout, qu'elles constituent des masses distinctes et séparées.

Telles sont les dispositions que l'on doit chercher à réaliser pour assurer la marche des coupes dans chaque affectation. Je, recommande surtout de ne jamais scinder une affectation, quand on n'a pas pour le faire des motifs majeurs.

La contiguïté des parcelles qui forment une affectation, n'est pas utile seulement pour l'application des règles d'assiette; elle l'est aussi pour l'économie des exploitations, lesquelles entrainnent nécessairement à leur suite des intérêts nombreux et un matériel considérable. Il est très-désirable que l'on n'occasionne pas à ces intérêts et à ce matériel des déplacements fréquents et coûteux. Or ces déplacements seraient inévitables si l'on formait, par exemple, une affectation avec des parcelles éloignées les unes des autres, et séparées par des massifs appartenant à d'autres affectations : après avoir fait des coupes d'ensemencement dans une partie de l'affectation, on pourrait être-obligé de les entreprendre dans une autre, puis de revenir dans la première pour les coupes secondaires, saúf à retourner l'année suivante dans la seconde:

Ce seraient là des inconvénients très-fâcheux, et la subdivision d'une affectation en deux ou plusieurs parties non contiguës, n'en présenterait pas d'autres, qu'ils suffiraient pour que l'on dût ș'efforcer de l'éviter; ainsi, il est clair qu'il n'importerait nullement, au point de vue de la régularité désirable dans 
la gradation des âges, que l'affectation de la première période ou celle de la dernière fût partagée en deux parties, situées l'une au commencement et l'autre à la fin de la série : une pareille disposition ne serait défectueuse que parce qu'elle contrarierait le principe d'économie ci-dessus énoncé; ce serait une raison suffisante pour ne paș l'admettre.

Les affectations sont enfin d'autant plus propres à faciliter, à assurer l'exécution de l'aménagement, qu'elles sont plus ramassées; car la tendance naturelle des agents est, dans l'assiette des coupes, de procéder de proche en proche, et il y àurait à craindre qu'ils ne négligeassent. souvent des opérations urgentes, s'ils étaient forcés pour cela de se transporter sur des points trop éloignés de ceux où auraient eu lieu leurs opérations précédentes.

On pourrait croire que l'application des règles d'assiette, au point de vue de la position respective que les affectations doivent occuper, trouvera presque toujours assez de garanties dans les dispositions arrêtées pour assurer la marche et l'assiette des coupes annuelles. Il ne suffit pas cependant que les règles soient observées dans l'assiette des coupes de chaque affectation, pour qu'il n'y ait rien à redouter de la non-observation de ces règles, dans l'assiette. des affectations; n'oublions pas, en effet, que nos futaies sont en grande partie situées dans les montagnes, où les accident's météoriques sont plus fréquents et beaucoup plus redoutables que dans les plaines; qu'elles offrent, par la hauteur des arbres 
qu'on y rencontre, beaucoup plus de prise que les taillis aux vents, à la neige, au givre; que la nécessité d'éclaircir les massifs que l'on veut régénérer, rend plus périlleuse encore leur situation, et que, dans de semblables conditions, la prudence exige quelquefois que les règles suivies pourl'assiette des coupes dans chaque affectation, le soient également pour le rang, l'ordre dans lequel ces affectations arriveront en tour de régénération. Il pourrait être dangereux, par exemple, de disposer les affectations de manière que les coupes se succèdent en allant du haut en bas d'une montagne ou dans tout autre sens qui les exposerait à l'action des ventș. L'expérience prouve, en effet, que l'on ne saurait prendre trop de précautions pour mettre à l'abri d'un coup de vent, les massifs à l'état de coupes secondaires ou de coupes d'ensemencement, et que la protection qu'offrent pour cela les bois de l'affectation dont elles font partie, n'est pas toujours suffisante.

Cette considération des obstacles à opposer aux vents, est celle dont on doit le plus se préoccuper dans l'exploitation des futaies en montagne ${ }^{1}$.

1: Quant aux forêts en plaine, c'est en outre aux gelées que l'on doit songer quand on règle la marche des coupes, car elles sont souvent la principale cause de l'insuccềs des repeuplements naturels ou artificiels, et cette cause motiverait une règle d'assiette non moins importante que celles indiquées par le cours deculture. Il n'y a pas un praticien qui n'ait remarqué que les coupes abritées du còté du nord et de l'est se repeuplent plus vite que celles qui sont ouvertes de ces còtés-là, et que lés semis yont une végétation plus active. (Note de la $2^{\mathrm{e}}$ édition.) 
J'ai exposé et discuté les raisons qui pouvaient contrairier l'application des règles d'assiette dans l'aménagement des taillis, et j'ai essayé de démontrer qu'elles n'étaient pas de nature à justifier la nonobservation de ces règles.

Quoique les pertes d'accroissement auxquelles on se condamne, quand on modifie le classement établi d'après l'âge d'exploitabilité, soient beaucoup plus considérables dans les futaies que dans les taillis, elles ne sauraient cependant prévaloir sur les inconvénients permanents qu'entraînerait une assiette vicieuse des coupes; car ces inconvénients sont de leur côté beaucoup plus graves dans les forêts de la première catégorie que dans les autres.

En fait, l'application des règles d'assiette, dans la formation du plan d'exploitation des futaies, n'est pas aussi embarrassante qu'on serait tenté de le croire.

Nos forêts ont été traitées, soit par la méthode à tire et aire, soit par la méthode jardinatoire : dans le premier cas, elles ont été exploitées de proche en proche; la gradation des âges y est donc assez régulière; dans le second cas, elles présentent sur tous les points des arbres de tous les âges, formant des peuplements uniformes dans leur irrégularité, et il est par conséquent indifférent, au point de vue des. convenances de l'exploitabilité, de commencer les exploitations par un bout ou par un autre. Enfin, en montagne, les coupes ont presque toujours été faites comme le veut la quatrième règle, c'est-à-dire en 
commençant par les parties inférieures, et la raison en est que cette manière de procéder était la plus commode ${ }^{2}$.

Quoi qu'il en soit, toutes les fois qu'on se posera la question de savoir si une parcelle doit être rattachée à une affectation autre que celle dans laquelle son âge l'avait fait classer, on la résoudra en examinant si cette parcelle est assez étendue, pour qu'il ne soit pas nécessaire de combiner l'assiette des coupes à y faire, avec l'assiette des coupes des parcelles contiguës: Dans l'affirmative, on devra la maintenir dans la colonne où on l'avait d'abord placée; dans la négative, on n'hésitera pas à la colloquer dans la colonne qui comprend les massifs limitrophes.

On conçoit donc que les exigences des règles d'assiette puissent faire colloquer dans une affectation des bois plus jeunes ou plus âgés qu'il ne faudrait, si la formation de cette affectation devait être exclusivement subordonnée à. l'âge d'exploitabilité; la régularisation de la forêt et les grands avantages qu'on a le droit d'en attendre pour l'avenir, le veulent ainsi.

1. Je crois devoir prémunir les agents contre une erreur trop commune dans l'application des règles d'assiette à la formation des affectations, erreur qui consiste à croire que lorsque ces rògles veulent que les affectations se succèdent du nord au sud par exemple, il est nécessaire de donner le $n^{\circ} 1$ à l'affectation située à l'extrémité nord de la forêt, et le dernior rang à l'affectation située à l'extrémité sud. Or, on peut donner le no 1 à telle affectation qu'on voudra, pourvu que les exploitations marchent dans le sens voulu. (Note de la $2^{\circ}$ édition.) 
II semble que ce sont là des principes auxquels il n'y a rien à objecter, et pourtant que d'étonnement ne provoquent-ils pas tous les jours! II n'est pas rare de voir des forestiers, très-estimables d'ailleurs, se récrier très-sincèrement, lorsqu'on les invite à pratiquer une coupe d'ensemencement dans une futaie qui est plus ou moins éloignée d'avoir atteint son plus grand accroissement moyen. C'est qu'ils ne comprennent pas qu'au-dessus des besoins du peuplement dans lequel la coupe a été assise, il y a ceux de la forèt envisagée dans son ensemble, et ils voudraient exploiter, ils exploitent trop souvent ce peuplement de la 'manière qu'ils jugent la plus convenable, en égard aux circonistances présentes, sans examiner s'ils obéissent ainsi aux prescriptions de l'aménagement.

Ces erreurs sont fréquentes; elles proviennent soit d'un défaut de portée dans les vues, soit d'une étude incomplète de l'aménagement que l'on est chargé d'exécuter; il y a, dans tous les cas, d'autant plus à les redouter qu'elles ont souvent leur point de départ dans un fait sainement apprécié. Les praticiens principalement sont enclins à les commettre, parce qu'ils ont l'habitude de considérer les faits en eux-mêmes' ou dans leurs conséquences immédiates et locales: ils n'apprécient que ce qui est sous leurs yeux; les circonstances extérieures et médiates leur échappent.

Mais il est évident que si l'on ne consultait, pour se diriger dans le martelage d'une coupe, que l'état actuel du peuplement, on ne pourrait que per- 
pétuer le désordre dans une forêt, ou l'y mettre s'il n'y était déjà. Aussi ne connais-je pas, dans le contrôle que l'administration supérieure est appelée à exercer sur la gestion de ses, agents, d'objet plus important que celui qui concerne l'applicátion des plans d'exploitation adoptés par elle.

Les agents n'ont pas toujours l'intelligence des sacrifices qu'il convient de faire à la régularisation de la marche des exploitations. Quand ils ont une coupe à effectuer, ils se dispensent volontier's de porter leurs regards au delà de l'enceinte de cette coupe. Ils ne sont pas, d'ailleur's, plus que les autres hommes, inaccessibles aux tentations de la critique, et ils ont bientôt dit: On veut que nous fassions une coupe d'ensemencement ici; mais c'est absurde : les bois sont encore trop éloignés d'avoir atteint l'âge d'exploitabilité; ou bien: On nous condamne à sacrifier ce beau repeuplement, en nous empêchant de le dégager du massif qui le domine; mais cela n'a pas le sens commun, ce massif est exploitable, il faut se dépêcher de l'abattre. Et c'est par des considérations de ce genre qu'on bouleverse les aménagements, qu'on compromet l'avenir; et qu'en croyant bien faire, on ne fait pourtant qu'empirer les choses.

Revenons à notre classement.

Lorsqu'on a arrêté son opinion sur les règles d'assiette dont l'àpplication à la forêt que l'on aménage est nécessaire; lorsqu'on s'èst fixé sur les dispositions qu'elles réclament, on s'y soumet rigoureusement dans la formation des affectations, ce qui 
veut dire que l'on ne s'arrête ni au désaccord existant entre l'âge d'une parcelle et lè rang de la périodè à laquelle il y a lieu de l'affecter, ni aux sacrifices d'accroissement qui pourraient en être la conséquence.

Mais ce n'est pas nécessairement une raison pour que l'òn soit ensuite forcé de régénérer tous les bois, dans la période correspondante à l'affectation dont ils font partie. Je montrerai commént, dan's lá première révolution, on est amené exceptionnellement à régénérer, en même temps que l'affectation de la période dans laquelle on se trouve, des parcelles qui appar-

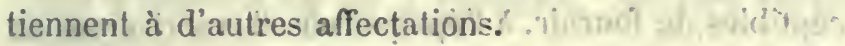

J'admettrai, jusqu'à nouivel ordre, que cette obligation ne doive pas résulter des modifications apportées au tableau des affectations, pour le rendré conforme aux règles d'assiette, et je vais voir quelles sont celles que le rapport soutenu pourrait de son côté motiver.

\section{$\$ 4$.}

Formation des affectations conformément aux exigences du rapport soutenu.

Toutes les parcellés qui; d'après leur âge, seraient exploitables dans la mêmé période, forment ce que l'on appélle une classe d'age. Unel forêt normale exploitable dans une révolution partagée en cinq périodes, doit donc comprendre cinq classes d'dgë, distinctes. Cette dénomination de classe d'âge; 
appliquée aux bois compris dans la même affectation, mérite d'être conservée, parce qu'elle est caractéristique.

L'aménagement a pour objet de rendre l'état respectif des classes d'âge aussi satisfaisant que possible. Pour qu'une forêt se trouve sous ce rapport dans toutes les conditions désirables, il faut : $1^{\circ}$ que les classes d'âge y soient en nombre égal à celui des périodes de la révolution; $2^{\circ}$ que la disposition de ces classes sur le terrain ne contrarie pas l'application des règles d'assiette; $3^{\circ}$ qu'elles aient la même puissance productive, en ce sens qu'elles soient susceptibles de fournir, à l'époque où elles arriveront en tour d'exploitation, le même volume.

Lorsque ces conditions n'existent pas, il s'agit de les étab!ir, et c'est précisément là le résultat que doit avoir le plan d'exploitation.

- Je ne laisserai jamais ćchapper l'occasion de rappeler ces principes, parce qu'ils doivent former la préoccupation constante de l'aménagiste, et que lorsqu'on ne les perd pas de vue, on est certain d'arriver au but.

Quand on a classé les parcelles dans les colonnes des périodes, en se conformant aux exigences de l'exploitabilité et des règles d'assiette, il suffit de jeter un coup d'œil sur les totaux de ces colonnes pour juger si la forêt que l'on veut aménager, est ou n'est pas susceptible d'un rapport périodique; et il n'y a pas non plus de longues réflexions à faire pour reconnaître si, ce rapport périodique étant 
réalisable, il est possible de le rendre soutenu, c'està-dire conslant.

Que mes lecteurs veuillent bien ne pas oublier que dans ce moment je ne m'occupe que des alfectations; que je les assimile à des coupes de taillis; que la période remplace l'année, et que, d'après l'hypothèse dans laquelle je me suis placé, le classement des parcelles, conformément aux règles d'assietle, n’a pas eu pour elfet d'augmenter l'écart qui doit normalement exister entre l'àge qu'ont les bois actuellement, et celui qu'ils auront lorsque l'affectation à laquelle on les a ratlachés, arrivera en tour d'exploitation.

Cela étant bien compris, si aucune classe d’àge ne fait défaut, on en conclura qu'il existe les élęments indispensables à une suile non interrompue d'exploitations périodiques. Dans le cas contraire, on avisera aux moyens de suppléer les classes d'àge absentes par des emprunts faits aux classes existantes, et c'est alors qu'il sera utile de se rappeler les règles que j'ai énoncées dans le chapitre relatif aux taillis.

D'après ces règles, des exploitations annuelles ne peuvent être entreprises et continuées sans interruption que lorsqu'il y a des parcelles exploitables, des parcelles assez jeunes pour atteindre, sans dépérir, le terme de la révolution, et des parcelles intermédiaires telles que la différence d'âge la plus grande entre deux peuplements à exploiter successivement, ne dépasse pas le double de l'écart tulérable entre 
l'âge d'exploitabilité et l'âge de l'exploitation, écart qui, dans aucun cas, ne doil être assez grand luimême, pour compromettre la régénération naturelle des massifs.

A l'aide de ces règles fơrt simpleś, on parvicndra sans beaucoup de peine à remplir, autant que possible, les lacunes qui s'opposeraient à la púriodicité des exploitations. Si c'est l'affectation de la première période, ou, en d'autres termes, la première classe d'âge qui inanque à l'appel, on pourra la remplacer par une partie de la deuxième classe d'âge et une partie de la dernière; si c'est la troisième classe d'âge, on pourra la remplacer par une partie de la deuxième et une partie de la quatrième; mais ces transpositions devront se faire de proche en proche, avec des peuplements contigus, de manière à ne pas déranger les dispositions prises conformément aux règles d'assiette, et à reculer, plutôt qu'à avancer, l'époque de l'exploitation effective des peuplements.

Ce qui manque ordinairement dans nos forêts, ce ne sont ni les bois exploitables, ni les bois des dernières périodes, ce sont les bois d'âge moyen. La substitution de la méthode du réensemencement naturel et des éclaircies périodiques au régime à tire et aire, substitution qui remonte à une cinquantaine d'années, est en partie la cause de cel état de choses. Dans les coupes de régénération auxquelles ils ont procédé, les agents n'ont pas toujours pris: les mesures nécessäires pour assurer le repeuplement naturel, et ils ont laissé s'accumuler les coupes d'en- 
semencement, dans l'altente d'un repeuplement que chaque nouvelle année de retard rendait plus improbable. Dans les coupes d'éclaircie, ils ont trop généralement cédé au désir de procurer des produits au Trésor, en faisant disparaître les vieux arbres de préférence aux jeunes. On ne saurait leur en vouloir, puisque l'absence de tout plan d'exploitation ne leur permettait pas de se guider dans ces opérations, d'après la composition et la distribution des classes d'ige, et qu'en outre, parmi les sujets qui rompent la régularité d'un peuplemeit, les plus gros sont naturellement ceux qui attirent le plus l'attention, et dont l'enlèvement paraît à priori le plus urgent.

Le rapport soutenu serait assuré par période, si les parcelles à exploiter dans chacune d'elles, c'està-dire les affectations de ces périodes, occupant d'égales contenances, renfermaient des massifs, similaires quant aux phases probables de leur développement, et gradués quant à l'àge, de manière à n'ètre ni plus ni moins âgés les uns que les autres, lor'squ'ils arriveraient en tour d'exploitation.

Mais ces conditions favorables ne se rencontrent jamais, et on doit dès lors se demander s'il ne convient pas de donner aux affectations des contenances iniégales, afin de corriger l'inégalité de puissance productive des parcelles qui les composent.

Les facteurs dont on se sert pour faire ces sortes de corrections ne sauraient, comme je crois l'avoir prouvé, se déduire de l'appréciation directe des élé- 
ments qui concourent à la production, tels que le sol, l'exposition, le climat, l'essence, l'état plus ou moins serré, etc. Ils se déduisent des renseignements que. l'on possède, sur la production effective de peuplements, placés dans les mêmes conditions de végétation que ceux de la forêt qu'on aménage.

Or j'ai fait o'sserver, en traitant du plan d'exploitation dans les taillis, qu'en allant à la recherche de ces peuplements comparables, on se lançait dans des difficultés d'appréciation très-nombreușes, très-délicates, presque insurmontables; et ce, pour n'obtenir souvent qu'un très-mince avantage, et perpétuer dans les classes d'âge une fâcheuse inégalité.

J'ai donc émis l'opinion que les procédés indiqués pour rendre les contenances des affectations inversement proportionnelles à leurs facultés productives, avaient une valeur pratique fort contestable.

Je maintiens cette opinion.

Mais la question est grave : on me pardonnera d'insister. Il s'agit de détruire des préjugés enracinés, des illusions géníralement répandues.

Je veux prouver qu'à raison de l'incertitude des moyens d'appréciation dont on dispose, il convient d'adopter comme règle générale, pour les affectations, des contenances égales.

Je dirai ensuite dans quelles circonstances particulières et dans quelle mesure, les exceptions à cette règle peuvent être admises.

1. Des motifs qui doivent faire adopter en général, pour les affectations, des contenances égales. - Mon- 
trons de nouveau d'abord que les méthodes prétendues différentes, par lesquelles on a cherché à déterminer les contenances inversement proportionnelles à la productivité du lieu d'habitation, reviennent toutes à fixer par hectare le produit de chaqie parcelle pour le moment de son exploitation; à comparer ce produit à un terme commun, et à faire figurer les parcelles dans le plan d'exploitation pour des contenances qui soient, avec les contenances réelles, dans un rapport inverse de celui trouvé entre la production présumée de chaque parcelle, et la production adoptée pour terme commun de comparaison.

En effet, voici en quelques mots ce qu'indiquent les autcur's qui se sont occupśs de résoudre le problème dont il s'agit.

Ceux-ci veulent : $1^{\circ}$ que l'on détermine le volume acluel de chaque parcelle; $2^{\circ}$ qu'on y ajoute celui dont elle sera susceptible de s'accroître, en supposant qu'elle reste encore sur pied jusqu'au milieu de la période à laquelle elle est provisoirement affectée; $3^{\circ}$ que l'on porte ces volumes dans la colonne ouverte à ladite période; $4^{\circ}$ que l'on procède enfin à l'égalisation des totaux des périodes par des transferts de volumes de la colonne la plus riche dans celle qui l'est le moins, en ayant soin de tenir compte des. diminutions ou des augmentations que l'accroissement. pourrait subir par suite de ces transferts.

Ceux-là comparent la production présumée par hectare de chaque parcelle, au moment fixé pour son 
exploitalion, à une production arbitraire quelconque prise pour type et terme commun de comparaison; ils multiplient la contenance réelle de chaque parcelle par le rapport numérique qui résulte de cette comparaison; ils substituent ces contenances fictives aux contenances réelles dans les colonnes des périodes, et ils procèdent enfin à l'égalisation des totaux de ces colonnes par des transpositions qui, au lieu de porter sur des volumes, comme précédenıment, portent sur des contenances.

Enfin, d'après d'autres auteurs, les éléments de la production d'une parcelle étant pris pour unité, on établit les rapports qui existent entre cette unité et la puissance des éléments de production des autres parcelles, et on fait de ces rapports le même usage que des rapports précédents.

Ces trois manières de procéder s'appuient évidemment sur la mệme base, et la première ne se distingue des deux autres qque parce que la production à laquelle on compare toutes les autres, est la production elfective moyenne au lieu d'être une production arbitraire.

Dans tous les cas, et c'est le seul point important, il s'agit de prévoir plus ou moins longtemps à l'avance le produit que donnera un peuplement lors de son exploitation. C'est là qu'est la pierre d'achoppement, 'qu'est la difficulté, difficulté qu'on peut dissimuler, mais qqu'on ne saurait éluder, et qui reste toujours aussi grande, quel que soit le moyen dont on se serve pour la surmonter. 
Ainsi, par exemple, quand, pour déterminer la production ultérieure de cliaque parcelle, on calcule : $1^{\circ}$ le volume actuel qu'elle contient; $2^{\circ}$ le volume dont elle s'accroîtra; on a recours à des moyens qui peuvent dissimuler, mais qui, en réalité, ne diminuent pas les difficultés inhérentes à cette détermination. Sans doute, le volume actuel est appréciable d'une manière suffisamment exacte, lorsqu'il s'agịt d'un peuplement dont tous les sujets sont assez forts pour être cubés individuellement. Si ce peuplement est âgé, on peut également, sans s'exposer à de grandes erreurs, se baser sur son accroissement moyen antérieur, pour apprécier son-accroissement futur; mais ce n'est pas pour les parcelles qui sont destinées à être exploitées prochainement, que la recherche du produit qu'elles donneront est embarrassante; c'est pour celles qui ont encore à rester longtemps sur pied; et, plus les bois sont jeunes, plus on risque de se tromper, soit dans l'estimation du volume actuel, soit șurtout dans l'appréciation de l'accroissement futur: dans l'estimation du volume actuel, par suite de l'impossibilité de procéder à un cubage individuel de tous les sujets qui composent le peuplement; dans l'appréciation de l'accroissement futur, parce que l'accroissement moyen acquis diffère d'autant plus de. l'accroissement moyen à acquérir, que les bois sont plus éloignés de la phase de leur plus grand développement, et que d'ailleurs, les chances d'erreur qui accompagnent les calculs sur l'accroissement, augmentent nécessairement avec 
le nombre d'années sur lequel portent ces calculs.

Aussi, pour les jeunes bois, est-on forcé de recourir à des tables de production ou à des peuplements exploitables et placés dans les mêmes conditions de végétation que ceux dont on s'occupe.

On n'échappe pas non plus à cette nécessité, lorsqu'au lieu de comparer les píoductions, on prétend arriver au même résultat en comparant les circonstances soús l'influence desquelles elles sont appelées à se réaliser. Si le lecteur veut bien se reporter aux observations que contient à ce sujet le chapitre sur les taillis, il y verra que cette prétention ne saurait être prise all sérieux que par los gens qui se payent de mots; que substituer la cause à l'effet dans l'énoncé du problème à résoudre pour déterminer la puissance productive des parcelles, ce n'est pas simplifier la question, c'est, au contraire, la compliquer sans faire disparaître aucune de ses difficultés; car, dans l'étude des phénomẹnes de la végétation, les causes ne s'apprécient que par les effets.

Voici un sol quelconque, imettez-le entre les mains d'un chimiste; il n'y découvrira pas, quelle que soit son habileté, la quantité de matière végétale qui pourra s'y développer dans un temps donné.

Une circonstance dont on fait grand cas dans l'examen de celles qui sont de nature à exercer une influence sur la production, c'est l'état de consistance du peuplement. Eh bien! je le demande à tout homme de bonne foi, quelle conclusion peut-on tirer de l'état de consistance d'un gaulis de quinze ans, 
relativement au volume qu'il présentera quand il en aura cent?

I! faut donc en revenir toujours à la comparaison des productions, par conséquenl, aux tables d'accroissement ou, à défaut, au cubage de parties de bais placées dans les mêmes conditions que celle qu'on envisage, et l'examen des éléments de la production n'a d'autre avantage que d'assurer le bon choix des peuplements choisis pour termcs de comparaison.

Quels que soient, en définitive, les moyens que l'on adopte pour la recherche du produit que donnera une parcelle à l'époque fixée pour son exploitation, le succès de cette recherche est fatalement subordonné à la découverte d'un peuplement exploitable, soumis aux mêmes influences que celui dont on veut ainsi prévoir la possibilité.

Or, cette découverte est extrênement difficile, et, avouons-le, presque impossible dans l'état actuel du peuplement de nos forêts. On cherclıerait peut-être vainement en France un seul massif exploitable, qui pût être présenté comme l'expression exacte de la puissance productive normale d'un terrain. Cela n'a rien d'étonnant, quand on songe que c'est à peine depuis une quarantaine d'années qu'on y applique le procédés d'une culture rationnelle.

On sait maintenant à quoi s'en tenir sur le mérite . des coefficients de production, sur l'utilité qu'on en peut retirer pour l'établissement dù rapport soutenu. Le rôle qu'on a voulu faire jouer à ces coefficients est, disons-le, une véritable illusion, qui 
prouve une fois de plus la funeste influence que peut avoir sur le bon sens et le jugement l'habitude des solutions mathématiques.

On est trop disposé de notre temps à transformer les questions en problèmes d'algèbre ou de géométrie; il y en a qui ne sont pas susceptibles d'une solution exacte, c'est un malheur auquel il faut se résigner. La question que nous traitons en ce moment est de ce nombre.

Il ne faut pas que la forme l'emporte sur le fond; que l'apparence soit prise pour la réalité. Ce fatras de calculs minutieux et compliqués dont on surcharge le tábleau des affectations, quand on veut tenir compte de toutes les influences qui réagissent sur la végétation, ne peut avoir de mérite qu'aux yeux de gens superficiels; il n'accuse en réalité que des prétentions à l'exactitude, et comme il se fonde sur des appréciations presque toujours arbitraires, on peut véritablement le comparer à un monument qui serait bâti sur le sable.

Au reste, si le rapport soutenu est une bonne chose; s'il est à désirer qu'on puisse l'obtenir pour chaque forêt, chaque série, il ne faut pas cependant s'en exágérer l'importance et oublier que, comme il intéresse surtout la consommation, c'est surtoul par bassin de consommation qu'il y a lieu de l'établir. Envisagées de cette manière, les atteintes qu'il pourrait éprouver sur un point, perdront beaucoup de leur gravité, si, comme il est probable, elles doivent trouver des compensations sur 'd'autres. 
On le voit donc : la réalisation du rapport soutenu n'est pas une condition rigoureuse qui demándé qu'on y sacrifie, sans motifs impérieux, la simplicité, la rapidité et la sûreté des opérations, et je conclus en conseillant, comme l'ont fait avant moi les auteurs de la Culture des bois, de donner autant que possible aux affectations des contenances égales.

2 Des molifs qui peurent faire adopter pour les affectations, des contenances réduiles. - Il n'y a pas de règle sans exception, et, dans les pays de montágnes surtout, il existe quelquefois, entre les circonstances' qui influent sur la régétation, des différences tellement tranchées, qu'on ne saurait les négliger.

Jusqu'à quel point est-il permis d'en tenir comple?

Ces différences peuvent être temporaireś. Elles le sont lorsqu'elles portent sur l'àge, la consistance, l'essence même, s'il entre dans les prévisions qu'elle devra être remplacée. Dans aucun de ces cas, je ne les crois, de nature à justifier une iriégalité dé contenances dans les affectations. Je l'ai dit pour les taillis, je le répète pour les futaies. Voici pourquioi :

Supposons que' l'on soit parvenu à se fixer súr lc rendement futur de chaque parcelle, à raison de toutes les circonstances, 'quelles qu'elles soient, dont ce rendement dépend. Supposons que, par' suite des différences de puissance productive qui existent entre ces parcelles, on ait jugẹ convenable, dans - l'intérêt du rapport soutenu, de transférer la parcelle $\mathrm{B}$, dont 
le coefficient est de 0,8 , de la troisième période dans la deuxième. Voilà une parcelle qui, par le fait de cette transposition dans une période qui avancera l'époque de son exploitation, mérite qu'on lui donne un autre coeflicient de production; car celui qui lui a été appliqué étant fonction de toutes les circonstances dont le rendement dépend, l'est dès, lor's, de l'àge d'exploitabilité, ou mieux, du temps pendant lequel ladite parcelle avait été destinée à rester sur pied. En conséquence, si on l'a fait figurer pour $\mathbf{X}$ hectares, dans la troisième période, on devra la faire figurer pour $\mathbf{X}^{\prime}$, en la colloquant dans la deuxième, et la soumettre dès lors à un nouveau remaniement.

D'un autre côté, la régularisation de la forèt implique la fixation de l'étendue respective des affectations, c'est-à-dire des classes d'âge; et l'on conçoit que cette fixation ne saurait être définitive, si on la subordonnait à des influences essentiellement temporaires. Ces influences n'existant plus, il deviendrait nécessaire de modifier les affectations et de retarder, par suite, l'époque à laquelle leurs limites cesseraient d'être provisoires.

La production subirait enfin des atteintes inévitables, si, pour la rendre soutenue, on augmentait par de nouvelles transpositions, l'écart occasionné déjà par l'application des règles d'assiette, entre l'àge de l'exploitabilité et celui de l'exploitation.

Donc, en admettant que l'on parvînt, chose presque impossible, à recueillir des données exactes 
sur le rendement probable de chaque parcelle, lors de son exploitation, on ne pourrait les utiliser pour la réalisation du rapport soutenu immédiat, sans se condamner à des tâtonnements, à des remaniements sans fin, et dans tous les cas, à une diminution de production, c'est-à-dire à des résultats contradictoires avec le but que l'on se propose lorsque l'on aménage une forêt:

Cependant, dira-t-on, s'il se trouviit des affectations qui continssent la moitié de leur étendue en vides, et qui fussent productives, par suite, d'un rapport moitié moindre, n'y aurait-il pas lieu de leur donner une étendue un peu plus grande ? - Non, parce qu'après que ces vides auraient été repeuplés, il faudrait de nouveau changer la contenance des affectations, sous peine de retomber dans les inconvénients qu'on avait vculu éviter. Tout ce que l'on pourrait faire, serait de décider qu'exceptionnellement à la règle générale, pęndant la première révolution, certaines parcelles ne seront pas régénérées dans les périodes correspondantes; mais cet expédient implique l'adoption d'un plan provisoire, et j'ai annoncé que l'étude de ce plan serait l'objet d'un chapitre spécial.

J'insiste donc sur ce principe : les affectations ne doivent jamais être modifiées, par suite des différences accidentelles, et par conséquent temporaires, qui porteraient sur l'âge, la consistance et même parfois l'essence des peuplements.

Mais, dans les conditions de la végétation, il yen a 
qui pcuvent être considérées, comme immuables; ce sout, par exemple, celles qui tiennent au climat, à l'exposition, à la nature des essences, à la qualité du soll; et ces conditions sont quelquefois tellement différentes d'une parcelle à une autre, qué l'on ne saurait vraiment être autorisé à passer outré. Il est certain qu'une affectation située sur une rampe exposéelau midi, ne rapportera pas, toutes les autres conditions étant égales d'ailleurs, autant que celle qui occupera un vérsant septentrional; et qu'un massif reposant sur un 'sol substantiel, frais et profond, rapportera plus que celui qui crôitra sur un teirain maigrè, sec et sans profondeur.

On sait qu'il est de principe de composer, autant que possible, les affeclations, de manière que les bonnes parties compensent les mauvaises. On sait aussi qu'au moyen de la division d'une forêt en séries, on parvient souvent à éviter lés embarrás qu'occasionnent, dans la formation des affectations, les différences de ferlilité des parcelles. Néanmoins, on doit prévoir des cas où ces moyens seraient insuffisants pour corriger les grands écarts que présenteraient les rendements probables dés affectations.

Dans ces cas-là, mais dans ces cas-là seulement, on pourrait donner aux affectations, des contenances inversement proportionnelles a leur puissance productive, en s'aidant, pour établir ce rapport entre les. puissances productives, de tous les éléments d'appréciation que fourniraient les tables d'expérience, les observations directes, la tradition surtout. 
Pour ne point se tromper trop grossièrement dans une opération de ce genre, il faut être très-expérimenté et très-circonspect.

C'est surtout lorsque les différences dans les conditions de végétation, portent sur la qualité des terrains, que les erreurs sont à craindre. Il n'en est pas des sols forestiers, ne l'oublions pas, comme des sols agricoles. Ceux-ci sont bien connus; les relations qui existent entre leur nature et le produit qu'ils peuvent donner, sont bien établies ; on peut assurer que telle terre ne produira que duseigle, et qu'elle en produira en moyenne tant d'hectolitres. Pour les forêts il n'y a guère de mauvais sols, en ce sens que dans presque tous, les bois peuvent prospérer; tel terrain qui, dénudé et desséché, ne paraît propre à rien, est cependant absolument semblable, sauf l'humus et la fraîcheur, à celui qui porte une magnifique futaie.

On ne saurait donc ètre trop prudent lorsqu'on recherche l'influence que peut avoir la qualité du sol sur la production forestière Quant à l'influence de l'essence, du climat, de l'exposition, elle èst moins douteuse.

Ces réflexions faites, je n'ai qu'un mot à ajouter sur l'emploi des coefficients de production. Lorsque ces coefficients sont fixés, on multiplie la contenance réelle de chaque parcelle par le coefficient y relatif, et on porte le résultat de cette multiplication sur le tableau des affectations, qui est modifié en conséquence conformément au modèle suivänt. 


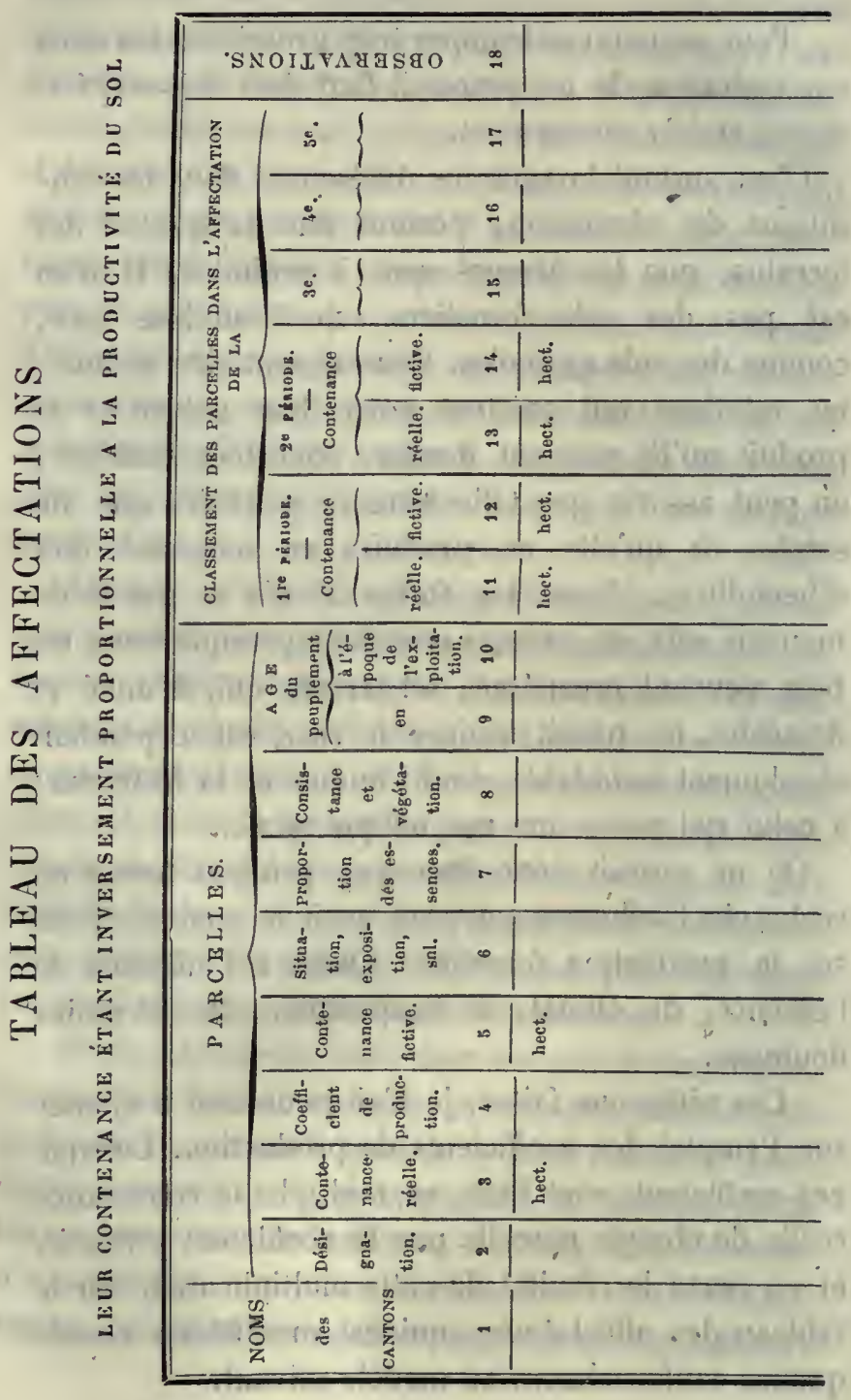


On procède à l'égalisation des totaux des contenances fictives, par des transferts, en se conformant aux préceptes que j'ai donnés, et on obtient ainsi, pour les affectations, des contenances réelles inversement proportionnelles à leur puissance productive.

\section{ARTICLE-II.}

RÈGLENENT GÉNÉRAL DES ESPLOITATIONS PAR PËRIOOE.

Quand la révolution principale peut ètre définitive, et le plan d'exploitation normal, le règlement des exploitations par période est peu compliqué. On ne change rien à la première partie du tableau des affectations, et l'on substitue dans la seconde partie, les mots parcelles à régénérer -lans la première, la deuxième, la troisième période, etc., aux mots classement des parcelles dans l'affectalion de la première, la deuxième, la troisième période, etc.; tous les bois compris dans la colonne affectée à la première période devrònt ètre régénérés, la révolution étant de 100 ans, et la période de 20 , dans les 20 premières années; tous ceux compris dans la colonne affectée dे la deuxième période, dans les 20 années suivantes, etc.

Mais les coupes de régénération, autrement dites coupes principales, ne sont pas les seules qu'il y ait à faire dans une futaie, et si, dans les taillis, les nettoiements et les éclaircies sont des opérations exceptionnelles et, dans tous les cas, peu productives, il 
n'en est pas de même dans les futaies. Ici, les, exploitations de l'espèce-ont une grande utilité, sous le rapport cultural, et sont en outre très-recommandables par les avantagès matériels qu'elles procurent immédiatement. 'C'est une première raison pour qu'on les fasse figurer sur le tableau des exploitations. En outre, une éclaircie, pour être bien faite, ne demande pas seulement que les agents d'exécution se conforment aux exigences actuelles, et à ce qu'on appelle les circonstances intérieures du peuplement; elle demande surtout qu'ils aient connaissance et qu'ils se préoccupent du rang que ce peuplement est appelé à prendre dans l'échelle des âges. Comme il est très-rare de rencontrer des massifs homogènes; comme les sujets de différents âges s'y trouvent ordinairement entremêlés, il importe beaucoup, pour qu'une éclaircie atteigne le but qu'on doit se proposer, que les agents d'exécution sachent quel est l'âge qu'il convient de faire prédominer dans un peuplement, et qu'ils connaissent en conséquence pendant combien de temps ce peuplement aura à rester sur pied, avant d'arriver en tour de régénération. Mais pour cela, il est nécessaire de faire figurer les nettoiements et les éclaircies sur le tableau des exploitations; il est nécessaire que ce tableau indique, sinon l'année précise, du moins la période de la révolution dans laquelle une parcelle devra être nettoyée ou éclaircie.

Cette indication n'est d'ailleurs nullement embarrassante: 
Les périodes embrassent presque toujours un laps de temps.plus considérable que celui qui, d'après les règles de la culture, doit s'écouler entre deux éclaircies successives; et il en résulte qu'il y a lieu de porter chaque parcelle dans toutes les périodes, soit pour être nettoyée, soit pour être éclaircie, à l'exception pourtant de la période dans laquelle elle aura été portée, pour être exploitée en coupe de régénération ${ }^{1}$.

Ainsi, la parcelle $\mathrm{R}$, qui figure sur le tableàu comme devant être régénérée dans la première période, devra y figurer en outre comme devant être nettoyée ou éclaircie dans les autres périodes. Seulement, pour éviter qu'on ne confonde, sur le tableâu des exploitations, les coupes d'amélioration avec les coupes principales, on a soin d'affecter ailx unes et aux autres, pour chaque période, une colonne spéciale, et le tableau des exploitations, par période, devient alors conforme au modèle ci-contre.

1. Cette exception ne s'applique pas toujours aux coupes do netloiement qui peuvent ètre nécessaires pour une parcelle dans la période même où olle a été régénérée. (Note de la 2 édition.) 


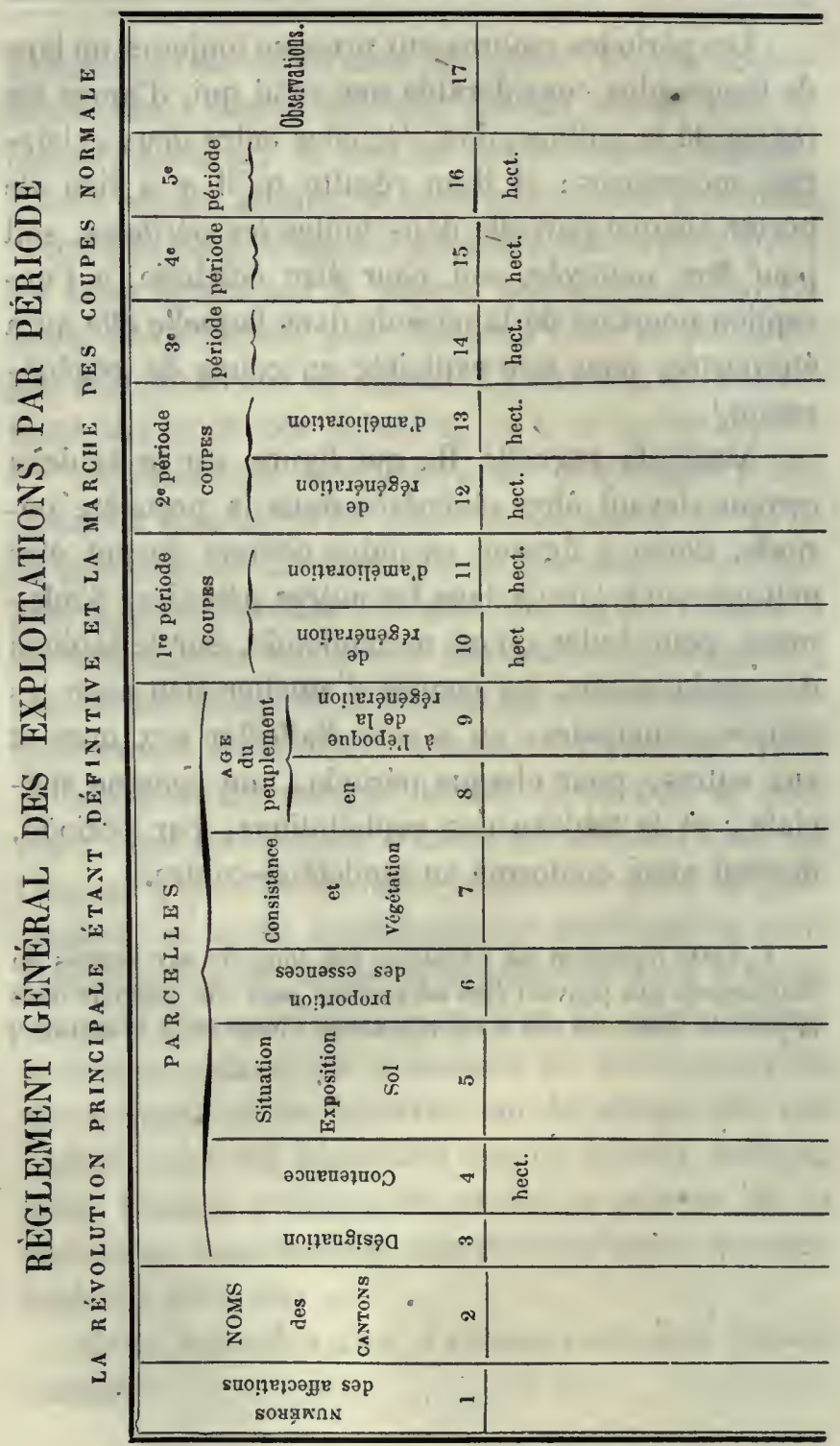


Comme nous avons supposé que l'état de la forèt permettait de fixer dès à présent et d'une manière définitive l'ordre des exploitations, en totalisant les contenances portées dans les colonnes du tableau, on devra trouver pour chaque période une étendue au moins égale à la contenance totale de la -forêt.

ARTICLE III.

RÉGLEMENT SPËCIAL DES EXPLOITATIONS AXNUELLES

PENDAXT LA PREMIÉRE PÉRIODF.

Coupes principales. - Si la forêt à aménager pouvait être exploitée à blanc étoc; s'il était permis de compter sur la régénération naturelle, malgré l'abatage, en une fois, de tous les arbres existants sur une contenance donnée; ou bien, si l'on trouvait économique de remplacer le repeuplement naturel par un repeuplement artificiel, et que ceiui-ci n'eùt besoin, pour réussir, d'aucun abri, le règlement des exploitations annuelles s'effectuerait en partageant chaque affectalion en vingt parties, ayant des contcnances égales ou inversement proportionnelles à leur fertilité. On suivrait, pour la division de chaque affectalion en coupes annuelles, les mêmes règles que pour l'aménagement d'un taillis.

- Mais, je l'ai dit, la nécessité de n'exploiter qu'en plusieurs fois les arbres existants sur un point donné, afin d'en assurer la régériération naturelle, s'oppose à 
l'adoption de cette manière toute simple de procéder; et c'est ici que l'aménagement des futaies exige des opérations qui n'ont aucune analogie avec celles que comporte l'aménagement des taillis.

Jusqu'à présent, tous nos elforts ont tendu, dans notre étude sur l'aménagement, à baser les exploitations sur la contenance, à cause de la simplicité, de la rapidité et de la sûreté que cette méthode est de nature à imprimer à l'assiette des coupes. Nous - sommes forcé maintenant de recourir à une autre base, car nous essayerions vainement de concilier la possibilité par contenance avec les exigences du rapport soutenu et de la régénération naturelle. On l'a tenté cependant, et il paraît qu'on y a presque réussi ${ }^{1}$; mais, sans nul doute, cette expérience a eu lieu dans des conditions tout à fait exceptionnelles, et l'on ne saurait s'en prévaloir quand il s'agit de poser des règles d'une application générale. On conçoit, par exemple, que dans une futaie de hêtres, reposant sur un sol substantiel, dans un climat tempéré, croissant enfin dans des conditions favorables à la fertilité des arbres de cette essence et à la germination des semences, on précise, sans s'exposer à de trop grands mécomptes, les époques auxquelles devront avoir lieu, sur un point donné, les coupes de régénération ${ }^{2}$. Le jeune plant de hêtre résiste long-

1. Voir les Annales de 1848, p. 158.

2. Dans certaines régions, à raison, d'une part, de la lenteur avec laquelle s'opère le réensemencement naturel, et de la faculté, 
temps sous le couvert. Les dernières éclaircies, pour peu qu'elles soient fortes, seront suivies, dans la forêt supposée, d'un repeuplement naturel qu'on 'trouvera en bon état, qu'on pourra utiliser, lor'sque arrivera le moment de procéder aux coupes principales; et que ces dernières soient faites un peu plus tôt ou un peu plus tard, en une ou plusieurs fois, cela est

d'autre part, qu'ont les essences de supporler le couvert, on a cru devoir régler les coupes d'ensemencement par contenance ct fixer la durée de la période à $\mathbf{4 0}$ ans, c'est-à-dire à un chiffre qui dépasse beaucoup celui que j'ai indiqué comme un maximun. Chaque affectation a d'ailleurs été partagée en deux parties cor. respondant à deus sous-périodes de 90 ans chacune, et tandis que l'on fait par volume des coupes secondaircs et définitires dans l'une des moiliés de l'affectation à régénérer, on fait par ' vingtième de surface des coupes d'ensemencement dans l'autre. M. Lorentz a publié à ce sujet, dans la dernière édition du cours de culture, un árticle auquel je renvoie mes lecteurs, en m.e bornant à l'observation suivante :

Il est certain que lorsqu'il n'y a pas d'inconvénient à fixer un délai constant entre les coupes d'ensemencement et les coupcs secondaires, il convient de régler les coupes de la première catégorie par contenance; car on ne doit, comme je l'ai déjả dit, recourir à la possibilité par volume qu'en cas d’absolue nécessité; mais la durée de la période n'est alors subordonnée qu'au temps indispensable pour effectuer sur le mème point les coupes secondaires et défnitives, et c'est une raison pour qu'clle soit plutòt diminuée qu'augmentée. Au surplus, dans la combinaison précitée, elle D'est augmentée qu'en apparence, attendu que ce qu'on appelle une sous-période est une période. Seulement, d'après cette combinaison, à la place des dernières éclaircies on doit faire des coupes sombres. Est-il bon, est-il prüdent d'y astreindre les agents d'exécution? Oui, je le répète, si on est sûr de pouvoir maintenir longtemss les jeunes repeuplements sous le couvert; non dans le cas contraire, et je crois que ce cas contraire se présente souvent. (Note de la 2 édition.) 
indifférent pour la régénération, qui sera déjà un fait accompli. Mais supposons une autre essence, le chêne pàr exemple, un climat plus rude, un sol moins fertile, et le repeuplement sera exposé à des éventualités qui ne permettront pas aux exploitations annuelles de suivre une marche régulière.

Il y a deux choses qui ne sauraient, en généra!, ètre prévues longtemps à l'avance et d'une manière précise, dans l'application de la méthode du réensemencement naturel : c'est d'abord l'assiette des coupes principales; c'est ensuite le nombre d'arbres à enlever dans chacune de ces coupes.

Un écrivain fort distingué, auquel le recueil des Annales forestières doit des communications trèsintéressantes, a essayé de prouver que la possibilité par contenance pouvait se concilier avec l'incertitude de l'assiette des coupes ${ }^{1}$. Mais, pour cela, il a été obligé d'admettre qu'il était possible de se fixer sur le nombre des arbres à enlever dans chacune des trois coupes de régénération. Ce nombre étant, par exemple, d'un tiers des arbres sur pied pour chacune d'elles, et la période étant de 20 ans, au lieu d'exploiter chaque année, en coupe rase, un vingtième de l'affectation des bois exploitables, on exploitcrait les trois vingtièmes, partie ici, partie là, en coupe d'ensemencement, en coupe claire ou en coupe définitive, selon l'élat des peuplements. S'il était admis que chacune des deux premières coupes

I. Yoir les Annales forestières du mois de septembre 1847. 
dùt prendie un quart des arbres sur pied, et la coupe définitive, le surplus, chaque hectare exploité, soit en coipe d'ensemencement, soit en coupe claire, serait considéré comme représentant un quart de la possibilité, cl chaque bectare exploité en coupe définitive comme en représentant la moitié. Pour compléter la possibilité, on aurait donc, chaque année, à parcourir une étendue telle que, mullipliée, selon la nalure de la coupe, d'ensemencement, secondaire ou définitive, par le facteur 0,5 ou 0,25 , elle produisit un nombre d'hectares égal au vingtième de l'affectation.

Tel est le moyen imaginé pour échapper aux prétendus inconvénients de la méthode d'exploitation basée sur la possibilité par volume. Ce moyen a été repoussé par des raisons très-calégoriques dans un article qui n'est pas signé, mais-dont il est facile de deviner l'auteur; car il est fait ex professo. Je renvoie à ce remarquable travail ceux de mes lecteur's qui auraient le désir de se rendre comple de tous les motifs qui rendent inapplicable au traitement des futaies, la possibilité par contenance ${ }^{1}$. Je me bornerai à faire observer ici que l'expédient inventé pour démontrer le contraire, serait de nature, lors même qu'il ne s'appuierait pas sur une lyypothèse inadmissible, à enlever à cette possibilité son principal mérite.

On ne saurait fixer pour un peuplement quelconque, le nombre d'arbres qu'il sera nécessaire ou

1. Voir les Annales forestières du mois de décembre 1847. 
utile de faire tomber dans chacune des coupes de régénération; et, pour s'en convaincre, il n'est pas nécessaire de recourir à des preuves tirées de la variabilité des conditions, dans lesquelles peut se trouver un massif, et des accidents imprévus auxquels la végétation est exposée. Il suffit de considérer qu'en réalité, si l'on en excepte la coupe définitive, les coupes de régénération, quelque faible que soit leur étendue, ont bien rarement un caractère tranché qui permette de les classer, soit dans la catégorie des coupes d'ensemencement, soit dans la catégorie des coupes claires. Elles participent presque toujours des deux : les unes ne sont que la continuation des autres, et on poursuit une chimère, quand on veut délimiter chacune de ces coupes, en préciser l'assiette et par conséquent la contenance.

Mais admettons l'hypothèse.

Je dis qu'en adoptant, pour les futaies, la possibilité par contenance, on ne rćaliserait aucun des avantages qui la rendent recommandable pour les taillis.

Ces avantages consistent, on le sait, dans la régularité, la simplicité, la promptitude, la sûreté des opérations.

- La régularité : il faudrait y renoncer, puisque l'assiette des coupes ne suivrait plus aucune marche certaine.

La simplicité : on ne pourrait point l'obtenir, puisque l'étendue à donner aux coupes annuelles devrait changer suivant la nature même de ces 
coupes, et qu'il serait nécessaire, sans parler de la difficulté de fixer la ligne précise de démarcation entre deux coupes différentes, de procéder à des arpentages multipliés.

La promptilude et la sûreté : il est aisé de prévoir ce qu'elles deviendraient en présence des incertitudes, des tâtonnements, des difficultés diappréciation et des travaux géodésiques que je viens de signaler.

- Il faut donc absolument trouver une autre base que la contenance pour régler les exploitations ammuelles, dans une futaie exploitée par la méthode du réensemencement naturel. Cherchons-la.

Si la forêt dans laquelle nous avons à faire cette découverte, forêt exploitable dans une révolution de 100 ans, se composait de 100 peuplements ne différant que par l'âge, occupant d'ailleurs d'égales contenances, composés des mêmes essences, -végétant dans les mêmes conditions; si l'on pouvait ajouter au matériel existant, celui que chaque peuplement serait susceptible d'acquérir ; n'est-il pas vrai qu'en divisant par 100 le volume total fourni par celte addition, on aurait pour quotient la quantité de bois dont la forêt s'accroîtrait annuellement, et, par conséquent, celle qu'on pourrait prendre cliaque année et perpétuellement, sans altérer sa puissance productive, en ayant soin toutefuis de ne jamais abattre que les arbres les plus vieux?

L'affirmative est évidente.

Le volume peut, en consćquence, servir de 
mesure aux exploitations annuelles, et, au lieu d'exprimer la possibilité en hectares, on peut l'exprimer en mètres cubes.

C'est exclusivement sur cette base du volume qu'ont été fondés les premiers aménagements de fulaies, lorsque l'ancienne et vicieuse méthode à tire et aire a été abandonnée.

Après avoir formé les affectations en classant les parcelles dans les différentes périodes, d'après les convenances de leur âge d'abord, et très-secondairement des règles d'assiette, on procédait à la recherche du matériel exploitable dans tout le cours de la révolution, et à cet effet, après avoir déterminé par des comptages individuels ou des places d'essai, le volume actuel de chaque parcelle, on calculait par les moyens plus ou moins sûrs qu'indique la dendrométrie, le volume dont elle était susceptible de s'accroître avant d'arriver en tour d'exploitation; mais comme on ne pouvait fixer d'avance, d'une manière certaine, l'année même de l'exploilation, on établissait les calculs comme si toutes les parcelles comprises dans une affectation, élaient destinées à être exploitées au milieu de la période correspondante ${ }^{1}$.

1. Supposons un produit qui aurait pour accroissement annuel l'unité, qui serait dès lors 1 la première année d'une période et 20 la dernière, le produit par anuée mosenne serait alors 10 , parce que tuus les termes d'une progression arithmëtique dont le premier terme est 1 et la raison 1 donnent pour la somme des 20 premicrs termes 210 , dont le vingtième, pour produit annueJ, 
Le volume total exploitable dans le cours de la révolution étant ainsi établi, il fallait voir s'il se partageait par porlions égales entre les diverses périodes : dans l'affirmative, il n'y avait aucun changement à y apporter, et en le divisant par le nombre d'années de la révolution, on obtenait le chiffre de la possibilité annuelle. Dans la négative, on procédait à l'égalisation des produits périodiques par des transpositions faites conformément auxirègles connues, et en tenant compte surtout de l'accroissement ou de la diminution des produits transportés d'une période dans une autre, atlendu qu'ils devaient nécessairement augmenter de tout l'accroissement que prennent les bois dont l'exploitation est retardée, ou diminuer de celui qu'ils ne peuvent prendre, lorsque leur exploitation est avancée.

Quand on avait effectué de cette manière l'égalisation des produits periodiques, et arrêté en conséquence le volume exploitable dans le cours de la - révolution, on obtenait le chiffre de la possibilité annuelle, en divisant ce volume comme on vient de le dire ci-dessus, par le nombre des années comprises dans la révolution.

Tel est en quelques mots, mais dans son objet principal et caractéristique, le système qui a été mis en vigueur, lorsque la possibilité par contenance ayant été reconnue vicieuse et inapplicable aux fu-

est $101 / 2$ ou, en nombres ronds, 10, movenne arithmétique des 20 nombres inégaux qui représentent les produits successifs s:ıpposés de la série des 20 produils annuels de la période. 
taies, on a cherché à y substituer celle par volume.

Mes lecteurs n'ont cerlainement pas besoin que je leur signale les vices de ce système, car les observations que j'aurais à faire à cet égard, seraient la reproduction textuelle de celles que j'ai développées à l'occasion des divers procédés mis en usage pour assurer le rapport soutenu. J'ai surabondamment démontré que celui de ces procédés qui est basé sur la production effective des parcelles, et qui a, par conséquent, pour résultat de déterminer la possibilité, n'avait aucune valeur pratique, à cause des incertitudes inhérentes au calcul de l'accroissement futur pour les massifs éloignés de l'époque de leur exploitation, et des erreurs monstrueuses dans lesquelles on est exposé à tomber en entreprenant un semblable. travail.

L'engouement pour la possibilité par volume, calculée pour toute la durée de la révolution, a pourtant été général à un cerlain moment, et tous les agents forestiers n'en sont peul-être pas encore dégagés. C'est un grand malheur; car, quelle que soit l'habileté de l'opérateur, quelques soins qu'il prenne pour assurer le succès de ces travaux, il ne peut aboutir (ju'à des déceptions. L'aménagement de la forêt de Ribeauvillé, exécuté en 1835 par les élèves de l'École forcstière, sous la direction du regrettable M. de Salomon, un de nos praticiens les plus exercés, l'a bien prouvé : les comptages et calculs effectués dans cette circonstance donnèrent des résultats qui ne furent pas justifiés par l’expérience. 
Mais si la supputation de la possibilité par volume, pour toule la durée de la révolution, est bien chanceuse, lors même qu'on y procède d'après toutes les règles et arec toutes les précautions qu'enseigne la science, il est aisé de comprendre qu'elle devient la plus inutile et, disons le mot, la plus absurde des opérations, lorsqu'on néglige, comme on l'a fait trop souvent, ces règles et ces précautions. Il n'y a pas de méthode d'aménagement qui dispense, par exemple, de l'établissement d'un plan d'exploitation, c'est-à-dire de l'obligation de déterminer, aussi étroitement que possible, l'assiette et l'époque des coupes. Pour calculer dans la méthode, exclusivement basée sur le volume, l'accroissement futur, il faut évidemment être fixé préalablement sur l'àge auquel une parcelle quelconque arrivera en tour d'exploitation; il faut un règlement de coupes, un tableau des affectations. C'est là cependant ce dont beaucoup de personnes ne paraissent pas se douter. Il fut un temps, et il n'est pas éloigné de nous, où presque de toutes parts, les agents forestiers se livraient à des dénombrements, à des cubages et à des appréciations de volumes futurs, sans réfléchir que ces travaux ne pouvaient aboutir à aucun résultat utile, dès qu'ils n'étaient pas fondés sur un parcellaire et sur un plan d'exploitation' 1 .

1. Ces fautes sont rares aujourd'hui, je m'empresse de le reconnaître. (Note de la ze édition.) 
Pour mettre un terme à ces déplorables errements, il serait à désirer qu'en attendant qu'elle pût publier une instruction sur les aménagements, l'administration défendît expressément de procéder à aucun inventaire sans son autorisation.

Reprenons la question du règlement des exploitations annuelles.

Les combinaisons difficiles, longues et compliquées, auxquelles on a eu recours dans l'aménagement des futaies, lorsqu'on a renoncé aux exploitations faites exclusivement par contenance, n'étaient pas, comme je l'ai montré, propres à remédier aux inconvénients de l'ancienne méthode; car si elles en supprimaient quelques-uns, elles en provoquaient d'autres non moins graves; et l'on peut dire que si, en théorie pure, elles apportaient un progrès réel à la science, 'en pratique, elles n'en réalisaient aucun. C'était un progrès réel que de substituer la possibilité par volume à celle par contenance; mais on le compromettait en prétendant pousser l'application du principe qui le caractérise, au delà des bornes posées par la nature aux prévisions humaines.

Qu'y avait-il donc à faire pour retirer de l'adoption de la possibilité par volume les avantages qu'elle offre, sans s'exposer aux inconvénients qu'elle pourrait entraîner?:

Il y avait à ne l'appliquer que dans les limites d'une absolue nécessité.

Ainsi, il n'est pas absolument nécessaire, les affectations étant formées, d'établir une règle pour 
les exploitations principales annuelles à faire dans les quatre dernières périodes, mais il est absolument nécessaire de l'établir pour les exploitations à faire dans la première.

Ramené à ces termés, le règlement des coupes annuelles ne souffre plus aucune difficulté, aucune complication, aucune incertilude.

On évalue le matériel exploitable;

On ajoute à ce matériel le volume dont il serait susceptible de s'accroitre dans un temps égal à la moitié de la période;

On divise le total par le nombre d'années de cette période, pour avoir la quotité des exploilations annuelles.

Le seul point qui, dans ces calculs, puisse exposer à des erreurs, est l'accroissement futur; mais comme il s'agit de bois parvenus à la phase stationnaire de la végétalion; que leur accroissement ne s'évalue, d'un autre côté, que pour un petit nombre d'années, on peut affirmer qu'qn ne saurait commettre une erreur sensible, en prenant pour mesurer cet accroissement, soit l'accroissement moyen des dix dernières' années, 'soit l'accroissement moyen de toutes les années qui forment l'àge des massifs ${ }^{1}$. Je n'insisterai pas sur ce point qu'il appartient à la den-

1. En général, on néglige tout à fait l’accroissement dans le calcul de la possibilite, et je crois que l'on a raison quand les périodes sont courtes, mais quand elles sont longues, quand leur durée est de 40 ans, par exemple, il est inadmissible que l'on ne tienne pas compte de l'accroissement futur. (Nole de la ze édi- tion.) 
drométrie de développer; je ne m’arrêterai pas non plus aux procédés de cubage à employer pour déterminer le volume des bois sur pied. Je me bornerai à recommander de déterminer ce volume en mètres cubes en grume, non-seulement pour la tige, mais pour les brainches, sauf à le décomposer ensuite en marchandises, d'après les habitudes du commerce.

Toutes les possibilités devraient être exprimées de la même façon, par la même mesure, le mètre cube en grume, qui est la seule précise ; et c'est une chose bien fâcheuse, qui occasionne beaucoup d'erreurs et, dans tous les cas, met du désordre dans la comptabilité forestière, que ce défaut d'unité qui existe encore dans la manière d'énoncer ces possibilités. Tel agent, cn se servant tu mètre cube, entend parler du mètre cube équarri au cinquième, tel autre du mètre cube équarri au quart, tel autre encore du mètre cube en grume. Les uns calculent la possibilité en mètres cubes, en stères et en fagots, les autres en stères seulement et en bourrées, etc., etc. Il ne serait pas moins raisonnable de la calculer en planches, en échalas, en merrains. Je le répète, il n'y a qu'une bonne mesure à employer : c'est le mètre cube en grume.

Coupes d'amélioration. - Les nettoiements et les éclaircies sont des opérations dont le produit immédiat est tout à fait secondaire, et dont le résultat pour l'amélioration des massifs est l'objet essentiel. Les principes rigoureux voudraient qu'elle fussent exclusivement subordonnées aux exigences de la végéta- 
tation, et qu'en conséquence on ne les soumît à aucune préfixion de temps, de contenance ou de volume; mais cet affranchissement complet de toute sujétion ne pourrait guère se concilier avec l'ordre et la régularité qu'il importe d'introduire dans de vastes exploitations dont les gérants se renouvellent fréquemment.

Qu'un petit propriétaire ne s'impose d'avance aucune règle pour le nettoiement et les éclaircies à faire dans sa forêt, on le comprend et on l'approuve, parce que son œil est ouvert ou peut l'être sur lous les points où de pareilles améliorations seraient appelées par l'état du peuplement; mais dans les forêts domaniales, si les éclaircies n'étaient l'objet d'aucune prescription, il en résulterait inévitablement qu'on négligerait souvent de les entreprendre, parce que les chefs de cantonnement en ignoreraient_l'urgence.

Il est donc utile.que les coupes de l'espèce soient prévues et prescrites, et par suite soumises à un règlement, et il ne reste plus qu'à chercher celui qui s'accorde le mieux avec les conditions -auxquelles elles doivent satisfaire.

Ce règlement ne saurait être basé sur le volume; en effet, pour fixer ce volume, toute base d'appréciation manque absolument. Élant donné un massif à nettoyer ou à éclaircir dans une certaine période de temps, il n'existe aucun moyen de savoir.ce que produiront ces opérations, et il serait d'un autre côté très-dangereux d'imposer à ce sujet un chiffre quelconque aux agents locaux; car ce serait détourner 
Léur esprit du but essentiel de leurs opérations et les exposer à sacrifier dans l'intérêt d'un produit immédiat, dont ils ne doivent pas du tout se préoccuper, l'intérèt de la conservation et de l'amélioration des massifs.

- Ir Un règlement basé sur l'étendue n'offre pas les mêmes dangers : sans doute, il arrivera que la contenance de la coupe sera tantôt moins grande, tantôt plus grande qu'il ne faudrait rigoureusement, parce qu'il n'est pas admissible, quelle que soit la régularité d'un peuplement, qu'il ait besoin, chaque année, d'ètre éclairci ou nettoyé par portions égales; mais, pour les éclaircies notamment, ce n'est pas une ou même plusieurs années de retard qui peuvent compromettre sérieusement l'avenir d'un massif. L'essentiel est que, dans la contenance désignée pour être éclaircie, les agents.aient la faculté de ne prendre que ce qu'ils jugeront convenable.

Ces principes n'ont pas toujours été pratiqués: dans l'application de la méthode d'après laquelle on calculait la possibilité, par volume et pour toute la durée de la révolution, le produit des éclaircies périódiques entrait dans cette possibilité, ce qui ne constituait pas la moindre cause des complications et des incerlitudes de l'opération; car s'il est difficile de calculer l'accroissement futur des bois, lor'squ'ils sont éloignés de l'âge d'exploitabilité, il ne l'est pas moins d'apprécier ce qu'ils pourront donner dans les éclaircies successives qu'ils auront à subir.

Toutefois, on comprend, au moins en théorie, 
que les forestier's qui n'avaient pas reculé devant les calculs d'accroissement pour les jeunes bois, aient osé aborder aussi l'évaluation du produit probable des éclaircies; mais ce que l'on ne comprend pas, et ce qui est pourtant, c'est que des agents qui avaient repoussé la pussibilité par volume pour les coupes principales à faire dans le cours de la révolution, aient .cru devoir adopter pour les éclaircies cette même possibilité. On a vu des projets dans lesquels toutes les éclaircies, quel que fùt l'àge des bois, reposaient sur la possibilité par volume. Ou en a vu surtout beaucoup où les éclaircies de l'affectation correspondáante à la deuxième période, étaieut réglées d'après cette base. On se décide évidemment avec peine à laisser dans l'incertitude, dans l'obscurité, le produit matériel; souvent considérable, des opérations de l'espèce, et pourtant c'est un sacrifice nécessaire, si l'on veut mettre chaque chose à sa place et subordonner l'accessoire au principal.

En conséquence, il convient de régler, par contenance, les coupes d'amélioration dans les futaies. Si donc les périodes sont de 20 ans, et s'il y a lieu de ne nețtoyer ou de n'éclaircir le peuplement qu'une. fois dans ce laps de temps, chaque affectation sera. partagée en 20 parties égales, pour être nettoyées ou éclaircies successivement dans chaque période, hormis celle dans laquelle elles devront être régénérées. S'il était utile de répéter les coupes plus souvent, tous les 10 ans, par exemple, chaque affectation serait partagée en 10 parties pour être nettoyées ou "éclair- 
cies successivement, deux fois dans chaque période, hormis celle dans laquelle elles devraient être régénérées, et une fois dans cette dernière.

On pourrait adopter un autre ordre et ne faire, par exemple, qu'une série de coupes d'amélioration, de sorte qu'au lieu d'être partagée en 10 ou 20 coupes, chaque affectation ne le serait qu'en 2,5 ou en 5 , ce qui simplifierait le service; mais avec cette combinaison, les produits intermédiaires ne sont pas soutenus, attendu que lés éclaircies rendent plus dans les massifs de 80 ans que dans ceux de 20, et, d'un autre côté, la surveillance générale de la forêt est moins garantie, les exploitations étant concentrées sur un plus petit nombre de points.

D'après les indications qui précèdent, pour former le tableau des exploitations annuelles dans le cours de la $\mathbb{1}^{\text {re }}$ période, nous n'aurons qu'à supprimer, dans le plan d'exploitation par période, les colonnes relatives aux $2^{\mathrm{e}}, 3^{\mathrm{e}}, 4^{\mathrm{e}}$ et $5^{\mathrm{e}}$ périodes, et à substituer dans celle de la $\mathbf{L}^{\mathrm{re}}$, aux contenances des parcelles à exploiter en coupes principales, les volumes desdites parcelles; puis, en totalisant les colonnes et en divisant chaque total par 20, nous aurons la possibilité, tant par volume, pour les coupes principales, que par contenance, pour les coupes d'amélioration, à exploiter dans le cours de la $\mathbf{I}^{\text {ro }}$ période.

Voir le tableau ci-contre ${ }^{1}$ :

4. Je pourrais supposer d'autres cas. Le lecteur, j'en suis sûr, n'en a pas besoin. Je dirai seulement encore qu'il n'est pas indispensable d'appliquer à toutes les affectations la mème périodicité 
DU PLAN D'EXPLOITATION.

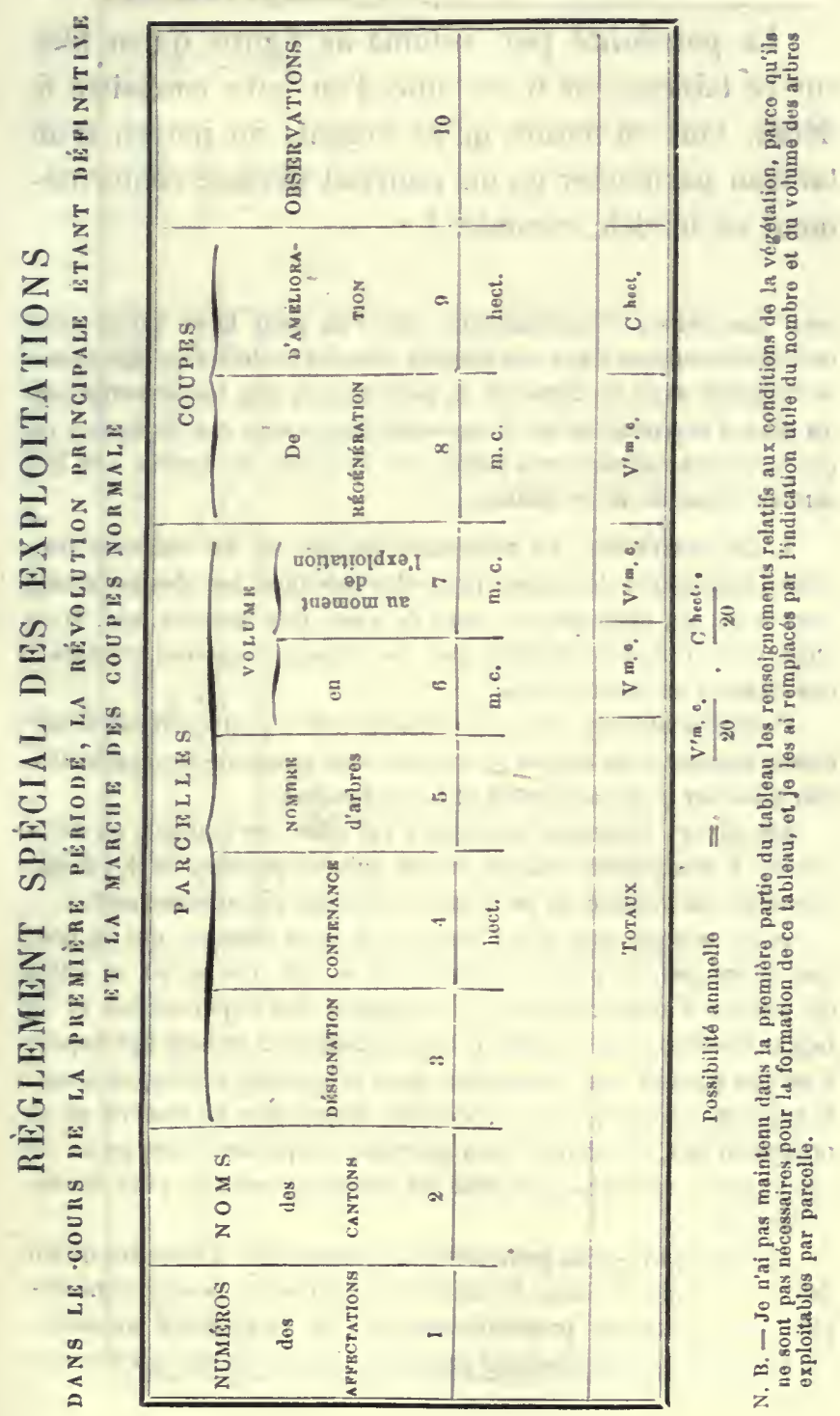


La possibilité par volume ne figure qu'en bloc sur ce tableau; or il est utile d'en faire connaître le détail, tant en nature qu'en argent, au moyen d'un tableau particulier qu'on pourrait dresser conformément au modèle ci-contre ${ }^{1}$ :

pour les coupes d'amélioration; que l'on peut faire varier cette périodicité suivant l'âge des massifs (pourvu toutéois qu'elle puisse se concilier avec la durée de la période); et que les prescriptions du plan d'exploitation ne dispensent dans aucun cas les agents de proposer des nettoiements toutes les fois que le besoin s'en fait sentir. (Note de la $2^{\text {e édition.) }}$

1. La possibilité, en admettant qu'elle ait été calculée avec toute l'exactitude désirable, peut être modifiée par des accidents imprévus : un incendie, un coup de rent, des insectes, etc. D'un autre côté, il faut considérer que les propriélaires sont exposés à des besoins extraordinaires.

Piusieurs auteurs sont en conséquence d'avis qu'il est d'une bonne économie de mettre en réserve une partie de la possibilité, afin d'obvier à ces accidents et à ces besoins.

Les divers systemes proposés à cet effet, reviennent, en définitive, à n'exploiter chaque année qu'une portion, le $1 / 10$ par cxemple, du volume qu'on pourrait prendre rigoureusement.

Je ne suis pas lout à fait partisan de cette mesure, qui ne peut que compliquer le plan d'exploitation, et qui me parait en outre: dis nature à compromettre la régularité des exploitations et le rapport soutenu: la régularité des exploitations puisqu'elle expose à ne pas épuiser une affectation dans la période correspondante; le rapport soutenu, car si l'occasion d'employer la réserve ne se présentait pas, il faudrait bien pourtant l'exploiter, sous peine de prolonger la révolution au delà du terme reconnu le plus convenable.

Je crois qu'il serait peut-être plus raisonnable d'attendre qụ'un déficit se produisit dans le matériel exploitable, pour y remédier par une réduction proportionnelle de la possibilité annuelle.

- Cependant je ne voudrais pas qu'on inférât de ces paroles que 


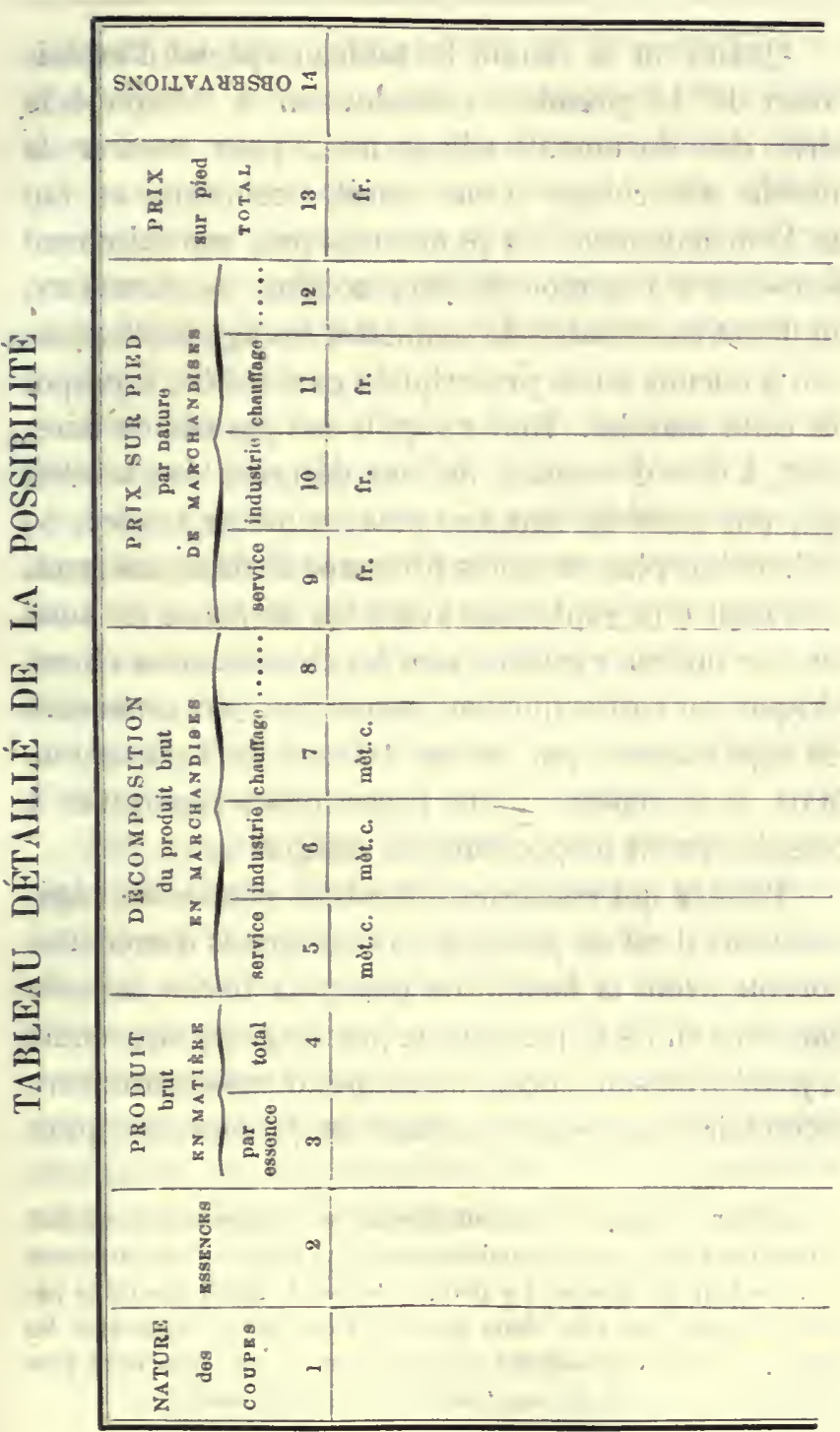


Quand on a rempli le tableau spécial d'exploitation de la première période, on a complété la série des documents nécessaires, pour assurer la marche des coupes d'une manière conforme au but de l'aménagement, et je ne crois pas, contrairement peut-être à l'opinion de bon nombre de forestiers, qu'il soit convenable de soumettre les agents d'exécution à aucune autre prescription particulière à propos de cette marche. Tout ce qu'il est permis de faire, c'est, à titre de conseil, de leur désigner les parcelles qui, par suite de leur âge plus ou moins avancé, ou de leur état plus ou moins prononcé de dépérissement, devraient être exploitées avant les autres; c'est aussi. de leur indiquer quelles sont les circonstances climatériques ou autres qui demandent que l'on commence les exploitations par tel ou tel côté de l'affectation. Mais, je le répète, toute prescription rigoureuse à ce sujet serait inopportune et dangereuse.

Pour ce qui concerne surtout les coupes de régénération, il est de principe que les agents d'exécution doivent avoir la faculté de porter la hache dans les parcelles où ils le jugeront le plus urgent, de prendre la possibilité sous forme de coupes d'ensemencement, secondaire ou définitive, dans les proportions qu'ils

je considère le rapport soutenu comme un dogme auquel on doit tout sacrifier. Je pense au contraire qu'il y a bien des circonstances où il ne faut pas hésiter à y porter atteinte. Il serait désirable par exemple que l'on pût, dans les forêts de chêne, augmenter les coupes après une abondante glandée, sauf à les suspendre plus tard, et, à ce point de vue, une réserve serait motivée. 
croiront les plus convenables; et l'on ne pourrait démontrer la convenance de leur imposer des obligations à cet égard, qu'en prouvant en même temps que la durée de la période n'a pas été fixée d'après les règles que j'ai exposées; qu'elle est plus longue, par exemple, qu'il ne faudrait, pour compléter la régénération naturelle de l'affectation correspondante.

Bien des gens se figurent que lorsque l'aménagement est fait, l'exploitation d'une forêt n'exige plus des agents locaux ni esprit d'observation, ni sagacité, ni intelligence. C'est là une grande erreur : pour se mouvoir dans le cadre très-large que leur fixe l'aménagement, pour se mouvoir avec le succès désirable, ces agents ont, au contraire, à accomplir une tâche difficile qui demande beaucoup d'activité, d'attention, et une expérience consommée.

Les coupes d'amélioration né réclament pas autant de liberté d'action que les coupes principales. Ces coupes peuvent, la plupart du temps, être avancées ou reculées de quelques années, sans inconvénients graves; il y a donc des cas où il serait sans doute avantageux d'en fixer l'assiette sur le terrain, soit en se servant pour cela des limites des parcelles existantes, soit en partageant par portions égales et régulières chaque affectation. 
Avant de m'occuper du plan d'exploitation dans les futaies qui ne comportent pas un aménagement normal, qu'il me soit permis de rendre hommage aux forestiers qui ont les premiers posé les bases de la méthode simplifiée dont je viens de développer le mécanisme et les précieux avantages : l'aménagement par case', c'est-à-dire fondé sur la possibilité par volume, calculée pour toute la révolution, était seul pratiqué et désespérait l'administration et les agents par des complications et des déceptions de toute sorte, lorsque MIM. Lorentz et Parade ont exposé, dans leurs cours de culture, les principes qui devaient ramener la simplicité et l'ordre dans la gestion trèscompromise de notre sol forestier.

L'aménagement avait suivi, du reste, la marche labituelle de toutes les conceptions humaines : informes au début, elles passent presque toujours par une phase de complication; puis arrivent des esprits fermes et rigoureux, simplificateur's, qui les dégagent de/toutes les superfétations, de tous les rouages inutiles et leur donnent la valeur pratique. C'est ce qu'ont fait, pour l'amẻnagement, MM. Lorentz et Parade, et la science leur est redevable, sous ce rapport, comme sous beaucoup d'autres, d'un progrès qui est destiné à exercer l'influence la plus heureuse sur l'avenir de nos forêts. 


\section{CHAPITRE CINQUIÈME.}

DU PLAN D'EXPLoitation daNS lES FUtales,

LA REVOLUTION POUVANT ÊTRE DÉFINITIVE ET LA MARCIE DES COUPES DEVAXT ETRE PROVISOIRE.

\section{ARTICLE PREMIER.}

FORNATION DES AFFECTATIONS.

$$
\$ 1^{\mathrm{er}} \text {. }
$$

Ctilité de la formation immédiate des affectations normales.

Quand la révolution principale ést définitive, le plan peut être provisoire, en ce sens que, pendant la première révolution, les parcelles appartenant à une affectation quelconque, ne devront pas toutes être régénérées durant la pétiode correspondan'e.

Ces exceptions à la règle, qui ne s'étendent ordinairement qu'à des portions peu importantes de l'affectation, ne doivent exercer aucune inflaence sur la formation du tableau des affectations.

Ce tableau établi d'abord d'après l'àge respec- 
tif des parcelles, remanié ensuite conformément aux exigences des règles d'assiette, est arrêté enfin comme si chaque classe d'âge était complète, ou, si l'on aime mieux, comme s'il y avait harmonie parfaite entre l'état du peuplement de chaque affectation et le rang de la période correspondante.

En effet, lorsqu'on aménage une forêt, il est fort utile que la situation que l'on en a vue de réaliser, au moyen de cette opération, soit exactement déterminée; or elle ne saurait l'être que par le tableau des affectations.

Ce tableau est un cadre dans lequel il faut faire rentrer le plus promptemént possible, et par les procédés que la culture enseigne, les parcelles d'une forêt; c'est un casier dont chaque compartiment est destiné à contenir des bois d'un âge déterminé, une classe d'âge particulière; il est donc nécessaire que la place et la contenance de chacun de ces compartiments soient connues d'avance, afin que les agents forestiers puissent façonner le peuplement de manière qu'il s'y adapte.

J'ai eu souvent occasion de vérifier des procèsverbaux d'aménagement, dans lesquels on avait cru devoir insérer des prescriptions longuement développées sur le traitement à appliquer aux différentes parcelles, suivant qu'elles se trouvaient dans telle ou telle condition:

Le tableau normal des affectations rend ces prescriptions à peu près inutiles. Le fait seul du classement d'une parcelle dans une affectation indique 
suffisamment les fonctions qu'elle a à remplir, et par suite le traitement qui lui convient.

La formation immédiate du tableau normal des affectations a un autre avantage : elle évite pour l'avenir des remaniements et des travaux de refonte pénibles, embarrassants et coûteux.

Celte formation, dans l'hypothèse où nous nous sommes placés, sera donc faite d'après des considérations analogues à celles que j'ai développées dans le chapitre précédent et les parcelles recevront leur désignation définitive.

C'est pour le règlement provisoire des coupes que nous aurons l'occasion d'indiquer des prescriptions toutes particulières et exceptionnelles.

Au reste, ce règlement provisoire est de nature à faciliter singulièrement, comme je vais le montrer, la formation des affectations.

\section{§ 2.}

Des facilités que donne, pour la formation des affectations, l'adoption d'une marche provisoire, pour les coupes.

On ne doit se résigner à adopter un règlement provisoire pour les exploitations - cela se comprend - qu'en cas d'absolue nécessité, et, par conséquent, toutes les fois que les règles d'assiette ou les exigences impérieuses du rapport soutenu ne s'y opposent pas, on évite de classer dans une affectation, des parcelles qu'on ne pourrait pas régénérer dans la période 
correspondante. Mais il est très-rare que dans des forêts dont le traitement n'a jamais été subordonné à des vues d'ensemble, à un plan quelconque, on ne trouve pas des bouquets ou parcelles de vieux bois disséminés au milieu de jeunes perchis, et vice versa, et qu'on ne soit pas dès lors amené à comprendre dans la même affectation, des peuplements qui ne sauraient être régénérés dans la même période.

Il arrive donc presque toujour's qu'on est obligé de mettre dans la dernière affectation, avec des vides ou de jeunes fourrés, des parcelles de bois exploitables, ou bien dans la première, avec des bois mûrs, des vides et de jeunes repeuplements. Je ne saurais indiquer toutes les anomalies auxquelles on pourrait avoir affaire. J'en donnerai seulement quelques exemples.

Il est évident que lorsqu'on veut faire suivre à des parcelles la même destinée, il faut chercher celles dont les âges se rapprochent le plus; mais toutes les fois qu'on est obligé de renoncer à soumettre, pendant la première révolution, au même traitement, des parcelles comprises ou à comprendre dans la même affectation, il faut au contraire réunir celles dont les âges présentent l'écart le plus considérable; parce que c'est le moyen d'avancer le plus possible l'époque de l'établissement de l'état normal.

Ainsi, les jeunes fourrés qui appartiennent naturellement à la dernière affectation, peurent être compris dans la première; il en est de même des vides, car, si on les repeuple immédiatement, ils s'harmo- 
niseront avec les peuplements environnants, créés ou à créer, et ne nécessiteront plus d'exception pour lıur régénération ultérieure.

Les bois mûrs, c'est-à-dire !es bois exploitables, qui appartiennent à priori à la première affectation, seront bien placés dans la dernière, au milieu des fourrés et des vides; parce qu'en les exploitant dans la première période, ils seront remplacés par de jeunes semis qui ne feront pas disparate au milieu des massifs qui les entoureront.

On voit combien le tableau provisoire des exploitations est propre à faciliter la formation des affectations de la manière la plus convenable, pour la constitution ultérieure aussi parfaite que possible des classes d'Åge. Est-on embarrassé pour asseoir la première affectation, attendu que les massifs exploitables se trouvent divisés en deux parties, l'une située au commencement de la série, l'autre à la fin? Il n'y a pas d'inconvénient à classer l'une de ces parties dans la première affectation, l'autre dans la dernière; seulement, celle-ci devra être régénérée exceptionnellement, anormalement, dans le cours de la première période. Les parcelles qu'elle contient, après avoir été ainsi régénérées, le seront encore à la fin de la première révolution. Mais de même qu'une parcelle est susceptible d'être exploitée deux fois, de même il y en a qui peuvent ne pas l'être du lout, et, par exemple, celles composées de tout jeunes bois, qu'on aurait dû comprendre dans'la première et même dans la deuxième affectation. 
En définitive, on doit toujours avoir en vue l'état des affectations après la première révolution, et adopter les combinaisons les plus propres à le rapprocher de la régularité désirable.

Mais ce ne sont pas là les seules facilités que présente le tableau provisoire des coupes, pour former les affectations dans une forêt irrégulière; en voici une autre que je signale, parce que l'occasion d'en profiter est fréquente : il s'agit d'une parcelle de jeunes bois qui appartiendrait, non-seulement par son âge, mais aussi par sa position, à la quatrième affectation; malheureusement l'état abrouti du peuplement ou son origine (des rejets de souches). ne permettent pas de conduire ce peuplement jusqu'à l'époque où l'affectation arrivera en tour de régénération. Eh bien! on surmonte la difficulté, en décidant que cette parcelle sera coupée en taillis une ou plusieurs fois, jusqu'au moment où les rejets seront susceptibles d'atteindre la quatrième période. Il pourrait en être ainsi de toute une affectation ${ }^{1}$.

Enfin, lorsqu'il y a impossibilité de placer des vides dans l'une des affectations extrêmes, il est bon de stipuler que leur repeuplement, s'il peut être fait immédiatement, ce qui est désirable, le soit avec des essences qui, ayant moins de longévité que la masse

1. Voir à ce sujet le remarquable article que contient le tome III des Annales forestières, sur un nouveau mode de conversion des taillis en futaies, mode qui a été appliqué, pour la première fois, dans la forêt de Cerisy (Manche). 
de la forêt, puissent être exploitables à l'époque où l'affectation dont elles font partie, arrivera en tour de régénération.

Telles sont les règles que l'on doit observer pour concilier la formation des affectations normales avec les exigences des peuplements. Elles ne sont pas toujours d'une application facile, et la nécessité d'exploiter une parcelle dans une autre période que celle à laquelle elle devrait être régénérée normalement, ne saurait être prévue avec certitude longtemps à l'avance. Aussi, ne doit-on regarder comme obligatoires que les propositions de coupes anormales qui se rapportent à la première période, et doit-on se réserver pour les autres, de modifier suivant les circonstances, au début de chaque période, les prévisions du règlement général.

Il me reste maintenant à indiquer, pour le tableau des exploitations, le cadre qui paraît le mieux se prêter à la consignation des prescriptions que ce tableau comporte.

\section{ART ICLE II.}

RËGLEYENT GÉNÉRAL DES EXPLOITATIOTS PAR PÉRIODE.

$$
\$ 1^{\text {er. }} \text {. }
$$

Coupes principales ou de régénération.

Dans une forêt régulière, il n'y a que deux natures de coupes à prévoir : les coupes principales de 
futaie, les coupes d'amélioration, et les parcelles sont toujours régénérées dans la période correspondante à l'affectation dont elles font partie. Rien de plus aisé dès lors que de donner unt ableau qui permette, comme celui de la page 278 , de reconnaître la période dans laquelle un peuplement devra être régénéré ou amélioré, ef l'affectation à laquelle ce peuplement appartient.

Dans une forêt irrégulière, il y a trois natures de coupes à prévoir au lieu de deux : les coupes principales de futaie, les coines d'amélioration et les coupes de laillis; c'est déjà une complication. En outre, nous venons de voir (p. 306 et 307) que l'époque de la régénération d'une parcelle de futaie, qui dans une forêt régulière est indiquée par le numéro de l'affectation dont cette parcelle fait partie, peut avoir besoin, quand il s'agit d'une forêt irrégulière, d'une autre indication. Une parcelle affectée à la cinquième période, par exemple, devra souvent être exploitée sous forme de coupe principale, dans la première; une autre classée dans la quatrième devra, au lieu d'être éclaircie, être exploitée en taillis avant d'ètre régénérée; de sorte que si, se contentant, pour les coupes principales, du nombre de colonnes qui figurent dans le tableau normal p. 278, on classait ces parcelles dans les colonnes correspondantes à l'époque de leur régénération, il s'établirait inévitablement une confusion sur le point capital de savoir dans quelle affectation elles sont comprises.

Pour prévenir cette confusion et pour que les 
coupes de taillis puissent aussi se distinguer des autres, nous ajouterons donc deux colonnes à chaque période du tableau p. 278 : l'une pour les coupes anormales de futaie, l'autre pour les coupes de laillis. Nous placerons dans la première toutes les parcelles qui devront être l'objet de coupes anormales, par ce seul fait qu'clles seront à régénérer dans une autre période que celle qui correspond à l'affectation à laquelle elles appartiennerit; nous placerons dans la deuxième toutes les parcelles qui, au lieu d'être éclaircies, devront être exploitées en taillis.

J'ai exposé dans l'article précédent les règles à suivre pour préparer les bases de ce double classement. Cependant, parmi les difficultés que rencontre un tel travail, il y en a une, relativement aux coupes de futaie, que je n'ai pas examinée, et qui se présente trop. souvent pour que je n'en fasse pas l'objet d'une observation :

Nos forêts, quels que soient les soins et l'intelligence arec lesquels on les a traitées dans ces derniers temps, comprennent rarement des peuplements parfaitement homogènes; on y trouve presque toujours et presque partout de vieux arbres qu'il convient d'enlever dans un délai plus ou moins rapproché. Suffira-t-il qu'une parcelle renferme de ces vieux arbres pour qu'on la classe dans la colonne des coupes anormales principales, c'est-à-dire des coupes anormales de régénération en futaie? - Non, et voici sur le parti à prendre, sur le moyen de lever 
les doutes qui pourraient s'élever à cet égard, la règle que je crois bonne à suivre.

Si les vieux arbres en question ne sont pas nécessaires au maintien du massif; s'ils sont dans un état qui constitue une coupe de régénération, une coupe secondaire ou une coupe défnitive; s'il est manifeste qu'il faudra les enlever tous dans un certain délai, ne dépassant pas la durée d'une période, on les classera parmi les coupes anormales de régénération en futaie, parce qu'ils pourront être compris dans la possibilité par volume, laquelle, nous l'avons vu, ne peut s'appliquer qu'à ces coupes; mais s'ils sont épars, de différents âges; s'ils ne constituent pas un peuplement parfaitement distinct et indéperdant des massifs qui l'environnent; si l'on ne peut, en un mot, préciser le délai dans lequel il est utile de les faire disparaitre, on laissera aux coupes d'amélioration dont je vais parler tout à l'heure, le soin de les, enlever en temps opportun.

Je ferai observer de même, en ce qui concerne les coupes de taillis, qu'il serait sans utilité réelle de leur ouvrir une colonne spéciale, si elles ne devaient former que de rares et insignifiantes exceptions. Dans ce cas, on les comprendrait parmi les coupes d'amélioration, sauf à rappeler par une simple remarque, l'attention sur la manière de les exploiter, pour les conduire jusqu'à la période de régénération.

Il faut enfin se mettre à la place des agents qui auront à appliquer l'aménagement, de ceux qui auront à le contrôler, et ne multiplier les colonnes 
qu'autant que cela est nécessaire pour l'intelligence du plan d'exploitation.

\section{$\$ 2$.}

Des modifirations que le rapport soutenu pourrait faire apporter au règlement des coupes principales.

Dans quels cas et dans quelle mesure les exigences du rapport soutenu doivent-elles faire modifier le règlement des exploitations par période?

On comprend bien que les affectations ayant été formées dans l'hypothèse de l'état normal, le rapport soutenu est compromis dès qu'on est amené à régénérer des parcelles, soit avant, soit après l'époque à laquelle l'affectation dont elles font partie arrivera en tour d'exploitation; de sorte que, en additionnant dans le tableau provisoire, les contenances affectées à chaque période, on ne trouvera probablement pas toujours le même total.

Doit-on essayer de remédier à cette inégalité par des remaniements, par de nouveaux transferts plus ou moins considérables, et qui ne seraient motivés que par des circonstances temporaires?

Telle est la question qu'on peut se poser, et sur laquelle je vais donner mon avis.

En général, sauf de rares exceptions, le rapport soutenu, dans la formation du tableau provisoire d'exploitation, est une chose dont il ne faut pas se préoccuper; car on ne le pourrait qu'en s'écartant 
des principes que nous avons adoptés relativement à l'influence des circonstances accidentelles sur ledit rapport, et qu'en compromettant ensuite deux résultats également recommandables et qui consistent : le premier, dans l'établissement le plus prompt possible de l'état normal ; le deuxième, dans l'exploitation des massifs à l'âge de leur exploitabilité, résultats que toutes nos combinaison;, jusqu'à ce moment, ont eu pour but d'atteindre.

Voyons, d'ailleurs, quelles sont les périodes pour lesquelles il y aurait peut-être quelque utilité à modifier de nouveau, en vue du rapport soutenu, le plan d'exploitation.

Est-ce pour la dernière?

Non; car si l'on a observé les règles que nous avons posées, on y aura classé les vides, les jeunes fourrés et gaulis et les vieux bois, sous condition d'exploiter ces derniers dans la première période; or, quelque grands que soient les vides ou l'étendue occupée par les vieux massifs, si la régénération a lieu immédiatement, la classe d'âge pourra être considérée comme normale, et lorsque l'affectation arrivera, à la fin de la révolution, en tour d'exploitation principale, son rendement le sera également; c'est là tout ce qu'on peut lui demander; lui demander davantage, adopter des combinaisons qui permettraient d'en obtenir plus que la possibilité normale, serait évidemment contraire à tous les principes d'exploitabilité et de bonne économie.

La dernière période est donc désintéressée dans 
la question, et en vérité, ce ne sont guère que les trois premières, qui pourraient avoir besoin qu'on modifiâl leur contingent par un remaniement des coupes anormales. Raisonnons donc sur ces trois périodes.

On peut supposer, soit un déficit, soit un excédant dans la première période par rapport aux deux autres.

Le déficit est peu probable, attendu qu'une des règles à suivre dans la formation du plan provisoire, est de rejeter dans la première période tous les bois exploitables de la dernière et même de l'avant-dernière affectation, et que d'un autre côté, ce qui manque dans nos forêts, ce sont les bois de deuxième et de troisième période, plutôt que ceux de première. Dans tous les cas, pour qu'il pût y avoir lieu de reverser une partie des produits de la deuxième et de la troisième période dans la première, il faudrait que ces produits dépassassent le chiffre du matériel normal de ces périodes: car autrement, on n'établirait l'égalité des produits entre les premières périodes, que pour la rompre entre les dernières.

Dans l'hypothèse de l'excédant, il arrivera de deux choses l'une: ou bien le contingent de la deuxième et de la troisième période sera inférieur à leur matériel normal, ou bien il ne le sera pas. Dans le second cas, l'excédant de la première période constituera une surabondance, une acoumulation de matière qu'on aurait dû éviter, et qu'en bonne économie, on doit réaliser le plus tôt possible. Dans le premier cas, 
il pourrait certainement être utile de combler une partie du déficit de la deuxième et de la troisième période, avec l'excédant de la première. Il est certain, par exemple; que si la deuxième et la troisième affectation renfermaient de grands vides, il serait permis de désigner certaines parcelles ou portions de parcelles, les plus jeunes dans tous les cas de la première période, pour être exploitées dans la deuxième et la troisièmé; mais ce sont là des expédients auxquels on ne doit recourir que dans des circonstances graves; car, en définitive, lé résultat inévitable de ces transferts est une perte de produits matériels, puisqu'ils prolongent le maintien sur pied, des massifs qui en sont l'objet, au delà du terme de leur exploitabilité.

\section{3.}

\section{Coupes d'amélioration.}

L'époque à laquelle doit être faite une coupe d'amélioration, dépend ordinairement de celle à laquelle doit avoir lieu la coupe principale. La place occupée par un massif dans les colonnes du tableau d'exploitation, affectées aux coupes principales normales ou anormales de futaie, fera donc connaître dans quelles périodes il convient que le même massif soit amélioré. La parcelle $\mathbf{A}$ fait partie de la dernière affectation, mais sur le tableau provisoire, elle figure dans la 
colonne des coupes principales anormáles de futaie de la première période, parce qu'elle se compose de massifs exploitables; on la désignera pour être améliorée dans chacune des autres périodes, sauf la dernière.

Telle est la règle générale, mais il serait possible qu'une parcelle figurant dans la colonne des coupes principales anormales de futaie de la première période, eût besoin cependant d'être éclaircie dans la même période, si elle était composéc par exemple de jeunes perchis surmontés de vieux arbres à extraire le plus tôt possible. Dans ce cas, ladite parcelle figurerait dans chacune des colonnes affectées aux coupes d'amélioration, sauf toutefois celle de la période dans laquelle elle devrait être régénérée.

Des parcelles de la première affectation, qui se composeraient de jeunes bois destinés à n'ètre régénérés qu'à la deuxième révolution, seraient, par des motifs analogues, inscrites pour être éclaircies dans chaque période.

Enfin, il se pourrait que des parcelles ne figurassent que dans les colonnes affectées aux coupes de taillis. C'est ce qui aurait lieu si ces parcelles ne devaient pas être éclaircies avant leur maturité. C'est une affaire d'appréciation.

Voici le modèle du tableau des exploitations par période, tant principales qu'intermédiaires 


\section{RĖGLEM ENT GÉNÉRAL DES}

\section{LA RÉYLUTION PRINCIPALE ÉTANT DÉFINITI}

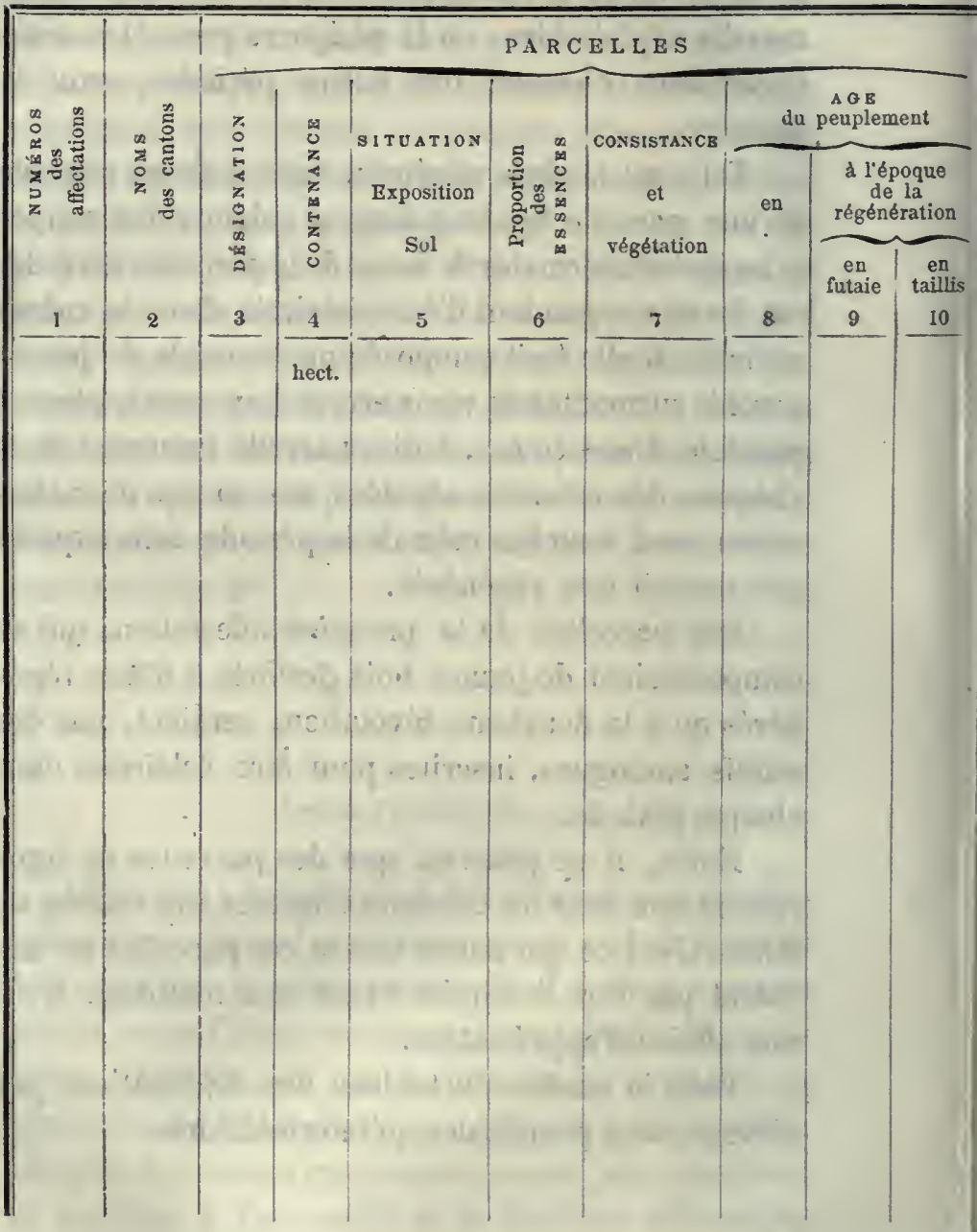

Nota. - Les colonnes des dernières périodes seront divisées et remplies commı 


\section{EXPLOITATIONS PAR PÉRIODE}

ET LA MARGHE DES COUPES PROYISOIRE

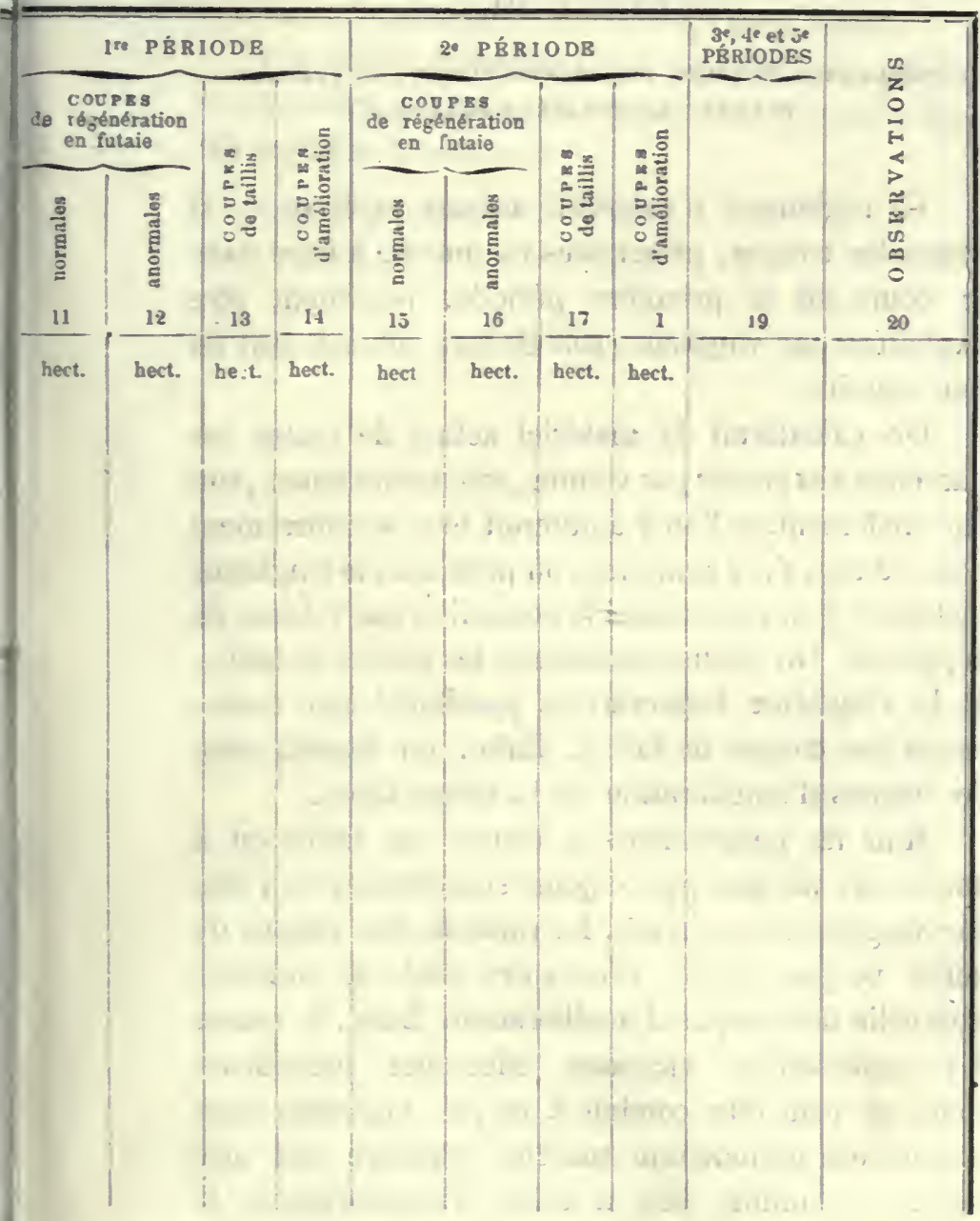

celles des périodes précédentes. 
ARTIGLE II.

RËGLENENT SPÉCIAL DES EXPLOITATIONS ANNUELLES PENDANT LA PRENIÈRE PÉRIODE.

Ce règlement n'exigerait aucune explication, si toutes les coupes, principales ou autres, à faire dans le cours de la première période, pouvaient être exploitées par vingtiène, soit de leur volume, soit de leur étendue.

On calculerait le matériel actuel de toutes les parcelles à exploiter par volume, soit normalemenl, soit anormalement, et l'on y ajouterait leur accroissement pour 10 ans, s'il y avait lieu; on prendrait le vingtième du total et l'on aurait ainsi la possibilité par volume de la période. On totaliserait ensuite les coupes de taillis, et le vingtième formerait la possibilité par contenance des coupes de taillis. Enfin, on fixerait celle des coupes d'amélioration de la même façon.

Mais les peuplements à couper en taillis ou à améliorer, ne sont pas toujours susceptibles de l'être par vingtième de surface. La rotation des coupes de taillis ne peut guère, d'un autre côté, se concilier avec celle des coupes d'amélioration. Enfin, à raison des exploitations vicieuses effectuées précédemment, on peut être conduit à ne pas imprimer dans la première période une marche régulière soit aux coupes de taillis, soit à celles d'amélioration. Il est donc nécessaire ou au moins utile, afin d'éviter 
la confusion, de dresser un tableau spécial pour les coupes par contenance, quelles qu'elles soient. Les tableaux spéciaux des exploitations, pendant la première période, pourraient être formés d'après les modèles ci-contre : 


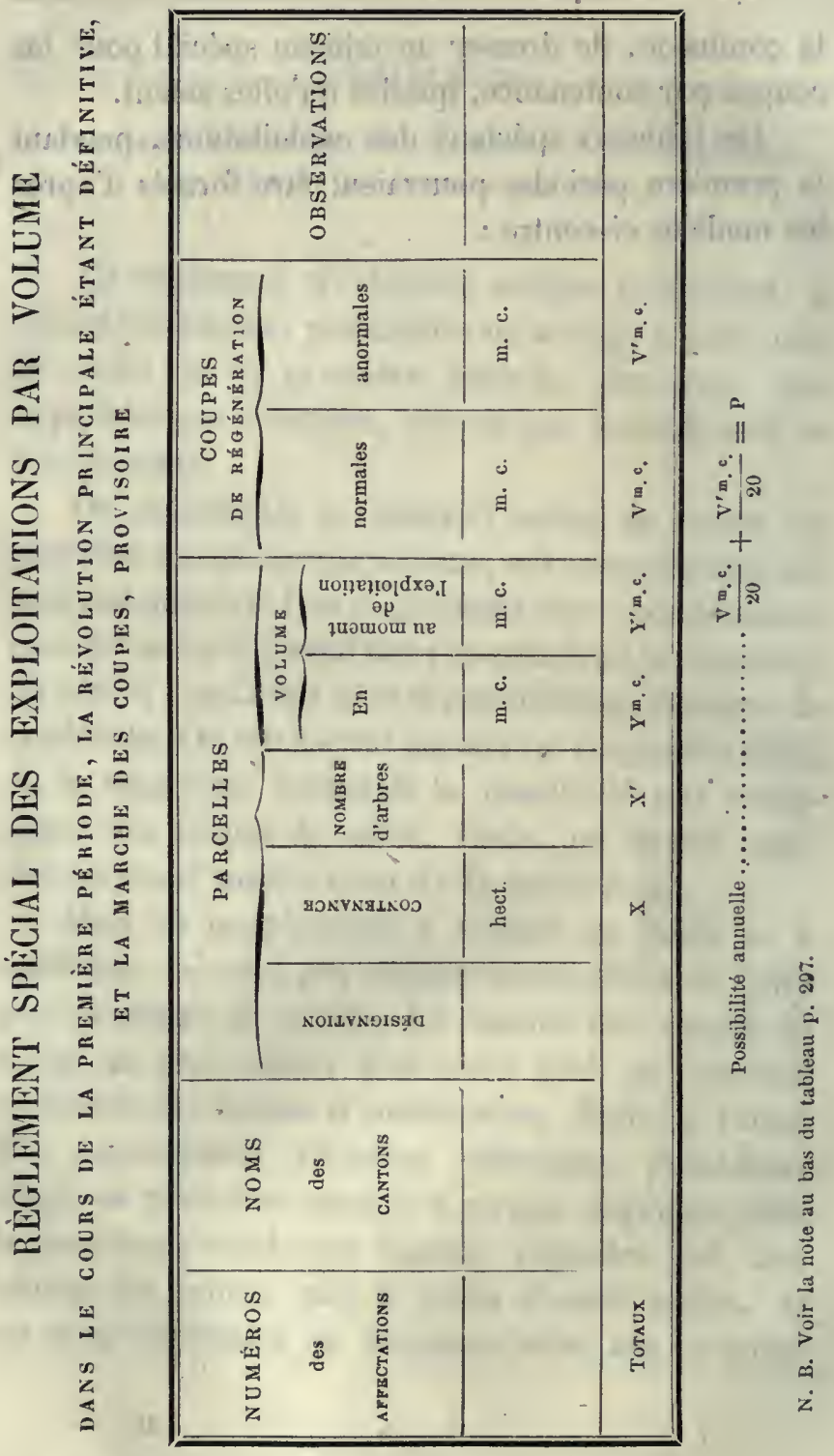


DU PLAN D'EXPLOITATION.

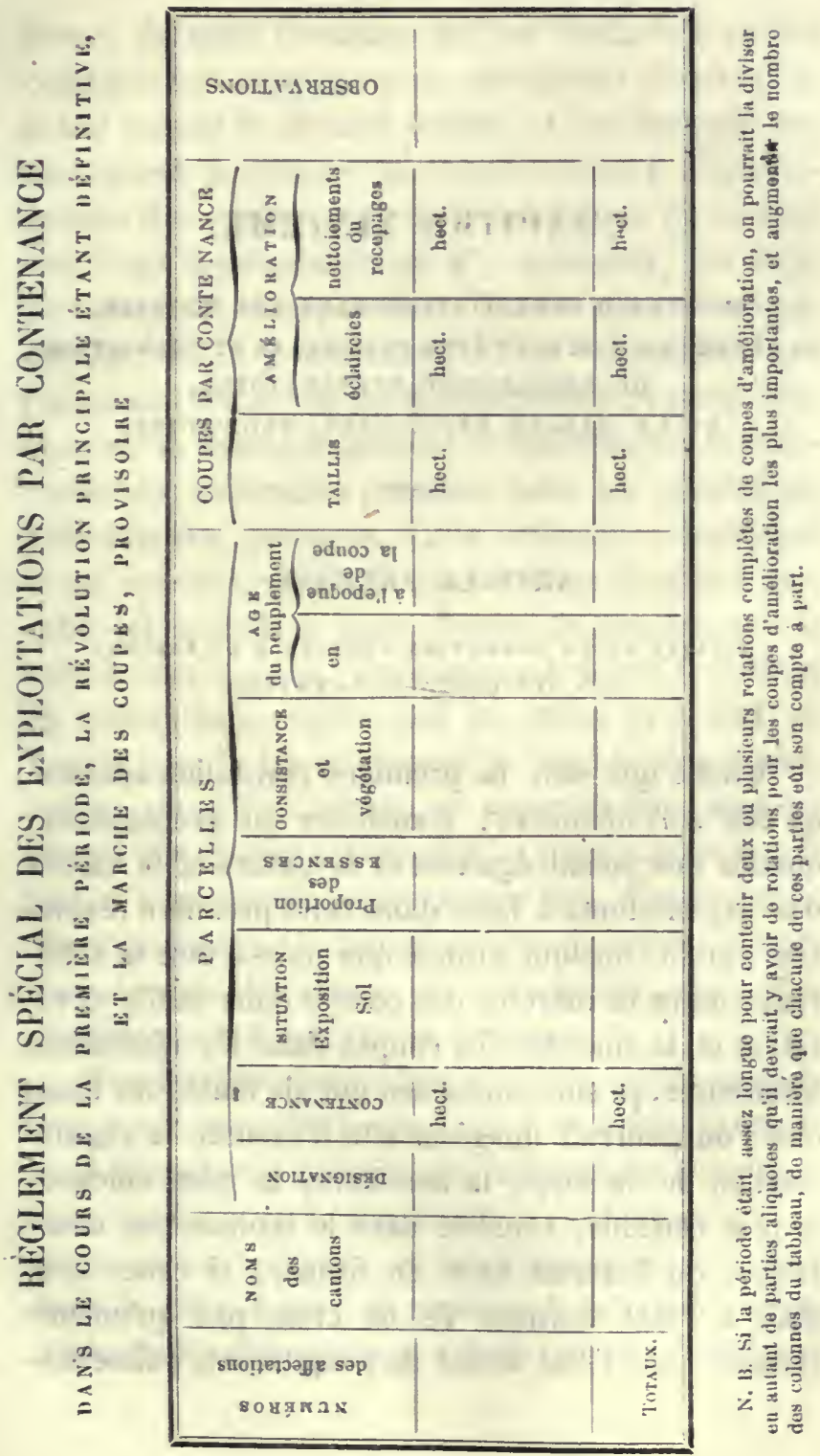




\section{CHAPITRE SIXIÈME.}

DU PLAN D'EXPLOITATION DANS LES FUTAIES, LA RÉVOLUTION DEVANT ÊTRE PRINCIPALE ET TRANSITOIRE, OU SIMPLEMENT PRÉPARATOIRE, F.T LA IARGHE DES COUPES, PROVISOIRE.

ARTICLE PREMIER.

UTILITÉ DE LA FORMATION PRÉLABLE DU TABLEAU

DES AFFECTATIONS NORYALS.

Quelle que soit la première révolution adoptée, qu'elle soit définitive, transitoire ou préparatoire; quelles que soient également la nature et la variété des exploitations à faire dans cette première révolution ; enfin, quelque grande que puisse être la différence entre la marche des coupes dans ladite révolution et la marche des coupes dans les révolutions ultérieures, je suis convaincu que de toutes les bases que l'on pourrait imaginer afin d'assurer la régularisation de la forêt, la meilleure, la plus efficace, la plus certaine, consiste dans le tableau des affectations qu'il serait utile de former, si cette forêt était à l'état normal. Je ne crois pas 'qu'aucune lacune, dans l'état actuel du peuplement, puisse dis- 
penser de cette formation qui est destinće à mettre constamment sous les yeux des agents d'exécution, le but auquel ils doivent tendre, et qui exempte par conséquent de remplir les procès-verbaux d'aménagement des nombreuses recommandations qu'on s'est cru jusqu'à présent obligé d'y consigner, au sujet du traitement des parcelles. Toutes ces recommandations, qu'on y réfléchisse, et on reconnaîtra que j'ai raison, toutes ces recommandations peuvent être, en général, avantageusement remplacées par la collocation des différentes parcelles dans les affectations normales des périodes. Cette collocation indiquant d'une manière précise la classe d'àge dont une parcelle est appelée à faire partie, la fonction qu'elle aura à remplir dans l'aménagement définitif, indique en même temps quels sont les soins qu'il faut lui donner pour la transformer de la façon la plus convenable; et si l'on admet, ce qui doit être, que les agents d'exécution connaissent les principes de la culture, il n'est pas nécessaire de leur dire, dans le cahier d'aménagement, la mantère dont ils auront à faire les balivages, les semis, les plantations, etc., pour atteindre le but que l'on se propose.

Une dernière observation achèvera, je le pense; de lever toute incertitude sur l'utilité et la possibilité de cette formation préalable des affectations normales: C'est qu'après tout, lorsqu'elle n'existe pas sur le terrain et sur le papier, il faut bien cependant l'avoir dans la tête, quand on procède à la régularisation d'une forêt, n'aurait-on à y faire que des éclaircies; 
car enfin, en réalité, quel que soit l'élat d'un massif que l'on veut aménager, l'aménagement consiste dans un travail de transformation qui peut sans doute être plus ou moins difficile, plus ou moins compliqué, plus ou moins long; mais qui ne cesse pas d'avoir le même objectif, de tendre exactement au même but et qui comporte en conséquence la même méthode et la même base. Cette base, c'est le tableau des affectations normales; cette méthode, c'est la subordination du caractère des exploitations, à la place qu'occupent les parcelles dans ledit tableau. Ce sont là des idées qui sembleront peut-être trop absolues, je le crains. Trop absolues, soit : nous faisons de la théorie, et par conséquent nous visons à l'absolu. Sont-elles justes? sont-elles vraies? sont-elles propres à apporter la lumière et l'unité dans les travaux d'aménagement? Telle est la question.

A mes yeux, les aménagements, proprement dits, les transformations, les conversions, rentrent dans le même genre d'opérations. Toutes ces opérations peuvent différer par quelques expédients; jamais par les moyens fondamentaux, et il me paratt nécessaire qu'on puisse reconnaittre, retrouver dans les plans qui les résument, les principes essentiels que nous avons essayé d'analyser.

Elı quoi! dira-t-on peut-être, vous voulez assujettir la transformation d'un massif composé d'arbres de 20 à 40 ans, entremêlés, aux mêmes règlements, au même plan que l'aménagement d'une forêt qui contiendiait toutes les classes d'âge nécessaires, pour 
qu'on pût y entreprendre et y continuer des coupes de régénération?

Oui c'est là ce que je demande, et je pense même que cela est indispensable pour que l'on ne soit pas privé du seul guide propre à assurer la marche de la transformation du massif que l'on vient de prendre pour exemple. En effet, cette transformation doit tendre, sous peine de manquer un des buts principaux de la culture des bois, à créer dans le massif en question, une gradation d'âge qui permette d'en retirer un jour le rapport soutenu le plus avantageux. Mais il s'agit alors de faire un aménagement ou d'en préparer au moins les éléments; or, pour cela, il faut évidemment se fixer sur l'âge d'exploitabilité, sur la durée de la révolution définitive, sur le sens dans lequel il importe que les classes d'âge se suivent; car, autrement, on ne saurait quels sont les sujets qu'il convient de conserver, ceux qu'il y a lieu d'abattre, etc. Bien que les opérations possibles actuellement ne soient que des éclaircies, on s'exposerait à compromettre par la manière de les effectuer, la formation des classes d'àge.

Admettez, au contraire, que la révolution définitive devant être de 100 ans el les périodes de 20 , on ait partagé le massif en cinq parties d'égale productivité, et indiqué la période dans laquelle il serait désirable que chacune d'elles pût être régénérée; alors, les agents d'exécution sauront, en procédant aux éclaircies : qu'ici il faut ménager les bois de 40 ans; que là on doit les sacrifier; et, quand 
arrivera l'époque à laquelle pourront commencer les coupes de régénération, la forêt sera susceptible d'un rapport annuel.

Le tableau des affectations normales étant arrêté, examinons comment on règle les exploitations dans les forêts auxquelles on est obligé d'appliquer une révolution principale transitoire ou une révolution préparatoire. ${ }^{1}$

On peut être conduit à adopter une révolution transitoire, lorsque l'âge trop avancé des massifs les plus jeunes ne permet pas de les laisser sur pied jusqu'à la fin de la révolution normale; et une révolution préparatoire, lorsque l'àge trop peu avancé des massifs les plus vieux ne permet pas de les exploiter immédiatement.

\footnotetext{
ARTICLE II.

DES RËGLEMENTS D'EXPLOITATION,

DANS LE CAS OU L'AGE TROP AVANCÉ DES MASSIFS

LES PLUS JEUNES, NE PERMET PAS DE LES LAISSER SUR PIED JUSQU'A LA FIN DE LA RÉVOLUTION NORMALE.
}

Quand on a partagé la révolution normale en un certain nombre de périodes, ouvert à chacune d'elles

1. Nous avons vu que l'âge d'exploitabilité n'était pas susceptible d'une détermination bien rigoureuse. Pour qu'une révolution puisse être qualifiée de transitoire, il faut donc qu'elle diffère beaucoup de celle que comporterait la longévité des essences. 
une colonne spéciale et classé, d'après les exigences de l'exploitabilité et des règles d'assiette, les différentes parcelles dont se compose la forêt, il est aisé de reconnaître dans quelle mesure les jeunes bois font défaut, et de déterminer par conséquent le temps dont il est nécessaire de raccourcir la durée de la révolution principale définitive, pour assurer la continuité des exploitations annuelles. Ce temps dépend des sacrifices que l'on est disposé à s'imposer sur l'accroissement, et surtout de la longévité des.. essences. La perte qui résulte du maintien des bois au delà de l'âge d'exploitabilité, est bien rarement assez manifeste pour motiver le raccourcissement d'une révolution. La longévité des essences est d'autre part assez grande, en général, pour que l'on puisse sans inconvénients, les laisser sur pied jusqu'au delà du terme fixé par leur exploitabilité; et ce n'est en conséquence que dans des circonstances exceptionnelles que se présentera l'impossibilité de remplir, avec l'excédant des classes d'âge les plus âgées, les lacunes que pourraient présenter les classes les plus jeunes. Admettons cependant que les deux dernières classes fassent défaut, et qu'il soit nécessaire d'y suppléer, au moins en partie, afin que les bois qui devront être immédiatement régénérés, aient le temps de redevenir exploitables sans trop de préjudice, pour le commencementde la révolution suivante; admettons d'un aulre côté, qu'à raison de la maturité des bois les moins âgés, il y ait danger à prolonger de plus d'une période le temps pendant lequel ils de- 
vraient, d'après leur âge, rester sur pied. Dans cette hypothèse, la durée de la révolution principale transitoire sera plus courte que celle de la révolution définitive d'un temps égal à la durée d'une période, et si la révolution normale est de $\mathbf{1 0 0}$ ans, la période de 20 , la révolution transitoire devra être fixée à 80 ans.

Cette fixation de la révolution transitoire est la seule opération embarrassante et délicate, dans l'étude du plan d'exploitation des forêts non susceptibles d'être soumises à une révolution définitive. Quand la révolution est arrêtée, on procède au règlement des exploitations, tant par période que par année, qui est destiné à compléter le plan d'exploitation, en suivant absolument les mêmes règles, que s'il s'agissait d'une forêt où toutes les classes d'àge seraient représentées ${ }^{1}$.

1. La période de la révolution transitoire étant de 20 ans, comme celle de la révolution définitive, et je ne vois pas de raison pour qu'il en fùt autrement, on régénérerait :

Dans la $1^{\text {re }}$ période de la révolution transitoire, la $1^{\text {re }}$ affectatation el le $4 / 4$ de la $2 \mathrm{e}$;

Dans la $2^{e}$ période de la révolution transitoire, $3 / 4$ de la $2^{\mathrm{e}}$, et $1 / 2$ de la $3^{\bullet}$;

Dans la $3^{*}$ période de la révolution transitoire, $1 / 2$ de la $3^{e}$ et $3 / 4$ de la $4^{p}$;

Dans la $4^{\mathrm{e}}$ période de la mûme révolution, $1 / 4$ de la $4^{\mathrm{e}}$ affectation et toute la 5 e. (Note de la 2 r édition.) 
ARTICLE III.

DES RÈGLEMENTS D'EXPLOITATION,

DANS LE CAB OU LAGE TROP PEC ATAXCE DES MASSIFS

LES PLES VIECX, SE PERMET PAS DE LES RÉGÉNÉRER

IM Y์ DIATEYEXT.

S'il y a rarement de grands inconvénients à laisser des bois dépasser l'âge normal de leur exploitabilité; si la limite d'àge supérieure jusqu'à laquelle on peut les pousser est difficile à préciser, on perd au contraire beaucoup à les exploiter avant leur maturité, et l'âge minimum qu'on peut adopter pour leur régénération, est celui auquel ils produisent des semences fertiles.

Il est évident par exemple que si, dans une forêt de bois feuillus, les peuplements les plus âgés n'avaient que 40 ans, il faudrait avant de les exploiter en coupes de régénération, attendre au moins 40 ans, et se borner pendant ces 40 ans, à les éclaircir.

Ce laps de temps est ce que j'appelle une révolulution préparatoire, parce que ce mot préparatoire indique bien la nature et le but des coupes à faire, avant que l'on puisse entamer lá régénération. Le réglement de ces coupes serą d'ailleurs facile à présenter : on divisera la révolution préparatoire en autant de parties qu'il faudra renouveler de fois la coupe préparatoire, c'est-à-dire l'éclaircie, sur un même point : en deux par exemple, si la rotation des 
coupes de l'espèce est fixée à 20 ans, et toutes les - parcelles à éc'aircir pendant chaque rotation, seront groupées en une série ou partagées en deux, suivant les circonstances. Je dis les parcelles à éclaircir, car il se pourrait qu'il y en eût à exploiter en taillis.

A l'expiration de la révolution préparatoire, la forêt sera dans des conditions analogues à celles que nous avons supposées dans l'article précédent.

Le modèle que j'ai donné, page 323, pour les coupes par contenance à faíre pendant la première période dans une forêt qui aurait toutes ses classes d'âge, servira à dresser le tableau général des exploitations pendant la révolution préparatoire. Quant au tableau spécial, il n'est pas nécessaire, à

- moins que la révolution préparatoire ne comprenne plus de deux périodes, et dans ce cas on prendra le même cadre, modifié conformément à la note inscrite au bas du modèle précité.

Si on a bien compris les exemples qui précèdent, on ne devra éprouver aucun embarras pour la formation du plan d'exploitation des forêts irrégulières, quels que soient les caractères particuliers qu'elles présentent. Les cadres que j'ai proposéș, se prêtent, je crois, et nous allons d'ailleur's en faire l'essai, à toutes les combinaisons auxquelles la conversion ou la transformation en futaies régulières peut donner lieu. 
Ces cadres me paraissent surtout recommandables, parce qu'il dispensent d'énoncer les règles de culture propres à assurer le succès de l'aménagement. Maintenant, qu'on y ajoute, si l'on veut, à propos de ces règles, quelques notes complémentaires explicatives, - je ne m'y oppose pas; abondance de bien ne. saurait nuire; mais on peut s'en passer, si l'on a affaire à des agents intelligents, instruils et observateurs. Dans le cas contraire, soyez sûrs que vos aménagements, de quelque façon que vous les exposiez, ne seront jamais exécutés. 


\section{CHAPITRE SEPTIÈME.}

CONVERION DES TAILLIS GOMPOSÉS EN FUTAIES PLEINES.

ARTICLE PREMIER.

OBSEHVATIONS PRÉLIMINAIRES.

La conversion des taillis composés en futaies a engendré plusieurs théories très-différentes, au moins en apparence. Elle n'implique pas cependant, comme je l'ại déjà fait observer, d'autres connaissances, d'autres appréciations, d'autres procédés, queceuxque réclame l'aménagement des forêts qui ont toujours été traitées en futaie. Lorsqu'on sait aménagel celles-ci, on est capable d'aménager celles-là, et la inéthode à suivre est la même pour les unes comme pour les autres.

Un taillis composé peut être assimilé à une forêt jardinée, en ce sens que des arbres de tous les âges y sont entremêlés; mais il offre cette particularité que les plus jeunes bois y sont représentés, dans chaque coupe, par des sujets du même-âge en massif plus ou moins compacte. A cause de cette particularité, le taillis composé semblerait devoir être moins 
irrégulier que la forêt jardinée, et cependant, en réalité, il l'est souvent beaucoup plus, par suite la différence d'âge qui existe entre les réserves. En effet, dans la forèt jardinée, les arbres de tous les âges ne sont pas toujours ensemble, et il est rare que l'on ne rencontre paś sur d'assez grands espaces des masses à peu près homogènes; tandis que, dans le taillis composé qui a été convenablement traité, les vieilles réserves, les anciens, les modernes et les baliveaur, sont véritablement pêle-mêle.

Toutefois, ce n'est point pour cela que les écrivains forestiers ont traité séparément de l'aménagement des futaies irrégulières et de la conversion des taillis composés : c'est parce que, dans les forèts de la première catégorie, les jeunes bois sont des brins de semence; tandis que, dans celles de la seconde, ce sont en général des rejets. Sans les rejets, le taillis composé rentrerait dans les conditions d'une futaie irrégulière, plus ou moins claire, et nous n'aurions pas à nous en occuper d'une manière spéciale; mais ces rejets constituent, pour la régénération naturelle, un instrument d'un genre tout particulier, puisqu'ils peuvent se reproduire autrement que par les semences.

La difficulté de la conversion des taillis composés en futaies, résideprécisément dans l'appréciation deces deux éléments, le taillis et la futaie, au point de vue du concours qu'ils sont susceptibles d'apporter à la régénération naturelle. Le concours du taillis peut être nul : c'est ce qui arrive lorsque ce taillis ne se com- 
pose que de bois blanes ou de morts bois; même lorsqu'il ne comprend que des bois durs, son concours peut n'être que secondaire : il en est ainsi lorsque les souches sont trop usées pour que les rejets en provenant, donnent jamais des semences fertiles. Le concours du taillis sera au contraire dominant, lorsque les rejets seront d'essence dure, vivaces, nombreux, et les réserves, rares. Enfin, ce concours sera de nature à enlever tout embarras aux combinaisons nécessaires pour amener l'état normal, lorsque le taillis sera tel que l'on puisse, sans en amoindrir sensiblement la qualité et la longévité, c'est-à-dire sans le rendre moins propre à l'ensemencement du sol, le couper encore plusieurs fois avant de s'en servir pour cette dernière destination.

Ces diverses considérations doivent toujours être présentes à l'esprit de l'aménagiste, lorsqu'il étudie un plan de conversion de taillis composé en futaie, et surtout quand il procéde au parcellaire; il n'oubliera pas non plus :

$1^{\circ}$ Que le premier principe de la culture forestière veut que l'on tire le plus de parti possible, pour la régénération d'une forêt, des ressources naturelles qu'elle renferme;

$2^{\circ}$ Que le mode du taillis ayant pour effet de dégrader le sol, plus tôt on y renonce et mieux cela vaut;

$3{ }^{\circ}$ Que l'intérêt de la culture, de la conservation et de l'amélioration des massifs, doit toujours primer celui qui se rattache à la production actuelle et au maintien du rapport soutenu; 
$4^{\circ}$ Que si le but que l'on a en vue quand on convertit une forêt, est d'y établir une gradation d'âge qui permette de retirer un jour de celte forêt des produits annuels plus avantageux que ceux qu'elle a donnés jusqu'à présent, il ne faut pas que le désir d'atteindre ce but un peu plus vite, fasse compromettre la régénération naturelle des massifs.

Le parcellaire des taillis composés est plus aisé, moins long à faire, en général, que celui des autres forêts; parce que les limites des coupes peuvent être conservées comme limites des parcelles, puisqu'elles séparent les peuplements dont les âges diffèrent, ce qui est ordinairement, comme on le sait, le caractère distinctif le plus influent; mais la description spéciale en est beaucoup plus délicate, plus embarrassante, parce qu'elle sert de base à des apprécialions fort incertaines et cependant nécessaires, comme par exemple celles qui se rapportent à la' vitalité des souches, à la longévité probable des rejets qui en proviennent, à l'âge auquel ceux-ci commenceront à donner des semences fertiles. Il est indispensable que l'aménagiste exprime sur ces divers points une opinion formelle, attendu que les combinaisons de l'aména gement en dépendent. Il est non moins essentiel qu'il s'explique sur la qualité des réserves de toute catégorie, sur le temps durant lequel elles pourraient rester sur pied, et sur le contingent qu'elles apporteraient au repeuplement naturel. Outre ces questions principales, la reconnaissance du taillis en soulève encore d'autres qui sont faites pour exercer toute la 
sagacité des meilleurs forestiers. Les bois d'élite, quoiqu'en minorité aujourd'hui, sont-ils assez nombreux pour former plus tard la majorité? Les jeunes brins de semence sont-ils en assez grande quantité et dans d'assez bonnes conditions, pour autoriser l'espérance qu'en les dégrageant par des nettoiements plus oı moins fréquents, on créera pour l'avenir des ressources sérieuses? La continuation des coupes de taillis seraitelle possible sans qu'on eût à en redouter de mauvaise conséquence, sinon pour le peuplement, du moins pour le sol?, Ce sol se prêterait-il à des rèpeuplcments artificiels? Il est désirable que la solution de toutes ces questions résulte de la description même des parcelles. Cette description exige donc du temps; de l'attention, de l'expérience, et même une sorte de prescience, un don de seconde vue, de divination, qui est d'ailleurs vraiment ce 'qu'on pourait appeler le sentiment, le flair forestier.

Ces préliminaires posés, abordons le problème de la conversion. Je n'aurai guère à me servir dans la discussion de ce problème que de termes précćdemment définis. Néanmoins, pour éviter toute équivoque, il n'est pas inutile que je précise encore mieux le sens que j'attache à quelques-uns de ces termes :

Quand je parlerai de coupes de taillis sous fulaie, à continuer temporairement, il s'agira de véritables coupes de taillis, telles qu'on doit les faire quand on veut obtenir une régénération par les souches, sauf la réserve qui pourra être sans dọte plus serrée 
qu'elle ne le serait d'après le premier paragraphe de l'article 70 de l'ordonnance du $1^{\text {er }}$ août 1827 , mais jamais assez pour entraver la production et le développement des rejets. Cette observation, sur laquelle j’aurai occasion de revenir, n'est par superflue; car, tout en conservant la qualification de coupes de taillis à certaines exploitations, on a souvent prescrit d'y prōcéder d'une façon qui devait en changer le caractère. C'est ce qui a eu.lieu, par exemple, pour les coupes de taillis dites modifiées dans lesquelles il était recommandé de 'réserver plu-, sieurs centaines de baliveaux; ou encore, pour les coupes de taillis dites de régénéralion dans lesquelles on espérait, par des dégagements, sauver assez de brins de semence pour en former plus tard une futaie. Cette manière d'appliquer un mode d'exploitation dont la pratique a été cependant consacrée par les règlements, par l'enseignement de l'école, et par la tradition, n’a pas peu contribué à égarer la discussion sur les conversious ${ }^{1}$.

Je rangerai sous la même rubrique : coupes d'amélioration, non-seulement les coupes préparatoires, les éclaircies, les nettoiements, les élagages, mais aussi les coupes à blanc étoc; les recepages plus ou moins complets, quand ces dernières opérations seront subordonnées à des circonstances éventuelles et à l'appréciation des agents d'exécution.

1 Le nombre des baliceaur à réserver, doit étre réglé de manière que le couvert quí en résulte, ne puisse compromettre la croissance et la reproduction du taillis (Cours de cullure, p. 376). 
Je repousse comme impropres les mots souspériode, sous-révolution, si souvent employés; car on ne saurait concevoir, en présence du sens que j'ai donné à la révolution et à la période, qu'elles puissent se dédoubler.

Enfin, je ne considérerai la conversion d'un taillis comme arrêtée, comme faite, que du jour où il aura été décidé que ce taillis ne sera plus recepé et devra se régénérer par les semences; jusque-là la conversion reste suspendue; de sorte qu'à mes yeux, le début de la révolution principale, normale ou transitoire, qui est marqué par le moment où on effectue la première coupe de régénération en futaie, peut avoir lieu sans que toute la forêt soit convertie ou, en d'autres termes, sans que toute la forêt soit destinée à ne plus être régénérée que par les semences.

\section{ARTICLE II.}

\section{UTILITÉ DE LA FORMATION PRÉALABLE DES AFFECTATIOTS} NORMALES.

La formation préalable des affectations normales est aussi utile pour la conversion des taillis composés, que pour l'aménagement des autres forêts irrégulières; et cette formation est plus facile en général pour la conversion, attendu qu'clle n'y est guère soumise qu'à une considération, celle des règles d'assiette. En effet, les exigences de l'exploitabilité perdent beaucoup de leur force dans les taillis com- 
posés, puisque les vieux arbres y sont uniformément répartis et que le taillis n'y présente pas des écarts d'âge assez grands pour que l'on ne puisse, sans des sacrifices sensibles d'accroissement, ranger dans une même affectation les plus âgés et les plus jeunes peuplements. Quant au rapport soutenu, de quelque façon qu'on s'y prenne, il est condamné à éprouvel une forte perturbation, aiusi qu'on le reconnaittra plus tard, et ce ne serait point par un changement dans l'assiette des affectations, qu'on y obvierait.

Ainsi donc, étant donné un taillis composé qui, après sa transformation en futaie, devrait être exploité dans une révolution de 141 ans, par exemple, et dont une portion quelconque pourrait être régénérée complétement en $\mathbf{2 4}$ ans, nous diviserons cette révolution en 6 périodes. Pour chaque période, nous déterminerons une affectation en nous conformant avant tout aux règles d'assiette, et nous remplirons le tableau indiqué page 244 , après avoir donné à chaque parcelle la désignation définitive que comportera l'affectation à laquelle elle appartiendra; mais on comprend tout de suite que, si on peut rarement imprimer immédiatement aux coupes une marche normale, même dans les forêts qui ont toujours été traitées en futaie, on le peut encore plus rarement dans les forêts dont on veut changer le mode d'exploitation; et nous avons à étudier quelles sont les mesures à adopter pour mettre ces forêts, le plus tôt possible et au mieux de tous les intérêts, dans les conditions désirables de régularité. Ces mesures 
doivent être résumées dans le règlement général des exploitations.

\section{ARTICLE-III.}

RÈGLEMEXT GÉNÉRAL DES TXPLOITATIONS.

Nous supposerons d'abord que nous avons affaire ì un taillis composé régulier et complet, riche en essences d'élite et en réserves de franc-pied. Ce taillis est soumis à une révolution de 24 ans; nous voulons le transformer en une futaie pleine exploitable dans une révolution de 144 ans, partagée en six périodes. Le tableau des affectations a été dressé conformément aux prescriptions des règles d'assiette; il s'agit de régler les exploitations par période. Je vais exposer les différents systèmes que l'on a suivis pour cela, et je dirai ensuite quel est à mon sens"le meilleur. Ces systèmes n'empruntent pas seulement leur originalité aux circonstances de la végétation; ils l'empruntent aussi à la rapidité plus ou moins grande que l'on est disposé à imprimer à la conversion : On peut se proposer de convertir immédiatement toute la forêt; on peut estimer au contraire qu'il est préférable de ne la convertir que graduellement. Dans ces deux cas, il y a bien des degrés de promptitude possibles; nous aurons à apprécier quels sont les plus avantageux. Enfin, que l'on veuille convertir immédiatement ou graduellement, la transformation pent se faire avec ou sans révolution préparatoire, et 
nous la discuterons dans l'une et l'autre hypothèse. Mais la supposition que nous fesons maintenant d'un taillis composé régulier et complet, se réalise exceptionnellement. Nos taillis sont en général irréguliers et incomplets et leur conversion est alors assujeftie à des règles très-variables. Nous en ferons l'objet d'un paragraphe spécial.

\section{$\$ 1^{\text {er. }}$}

Règlement général des exploitations d'un taillis composê régulier et complet a convertir, lorsque la révolution est préparatoire et la conrersion immédiate.

Puisqu'on veut que la conversion soit immédiate et générale, il fant arrèter les coupes de taillis sur toute l'étendue de la forêt et, par ce fait seul, on peut dire que la forèt est convertie; mais il s'agit d'y créer ce qu'on appelle des classes d'àge et c'est là le difficile: d'abord, parce que les taillis de la première affectation, en supposant même que ce soient les plus âgés, ne sont pas en général susceptibles de porter des semences fertiles; ensuite, parce que les taillis de la dernière, en supposant que ce soient les plus jeunes, ne sauraient fournir une aussi longue carrière que des brins de semence. Deux questions se présentent donc : la première, étant-de savoir pendant combien de temps on devra laisser sur pied les taillis de la première affectation, pour les amener à un état tel qu'ils soient à même de se régénérer naturelle- 
ment par les semences; la seconde, étant de savoir pendant combien de temps on pourra laisser sur pied les taillis de la dernière affectation, sans qu'on ait à craindre leur dépérissement.

Dè la solution donnée à la première de ces questions, dépend la durée de la révolution préparatoire. De la solution donnée à la seconde, dépend la durée de la révolution principale. Cette dernière révolution sera d'autant plus longue, elle se rapprochera d'autant plus de la révolution normale, que la révolution préparatoire sera plus courte; et la répartition des peuplements, la plus favorable au raccourcissement de la révolution préparatoire et à l'allongement de la révolution principale, serait naturellement celle qui aurait classé les taillis les plus âgés dans la première affectation, tandis que les plus jeunes appartiendraient à la dernière.

L'état des rejets, l'état des souclies, le nombre et l'état des réserves, la nature des essences, l'àge auquel elles portent semences, telles sont les circonstances à apprécier, afin de résoudre l'une ou l'autre des questions que je viens d'énoncer; seulement, les rejets auront moins d'influénce sur la solution de la première question que zur celle de la seconde, et cela se conçoit aisément, puisque dans la première affectation on a besoin de vieux bois, tandis que dans la dernière on ne saurait en avoir de trop jeunes.

Si on se croyait dans l'obligation de laisser croître les taillis jusqu'à l'âge de cinquante ans, afin que leur action, s'ajoutant à celle des réserves, pût assurer 
le réensemencement naturel, il en résulterait cetle conséquence forcée que, pendant un laps de temps égal à la différence entre l'àge de 50 ans et l'âge actuel des taillis, la forèt ne serait parcourue que par des coupes d'éclaircie, de régularisation si l'on veut, ou, pour me servir du terme consacré, par des coupes préparatoires dans lesquelles on s'appliqưerait à ménager, tout en évitant d'interrompre le massif : dans la première affectation, les vieux arbres; dans la dernière, les jeunes; dans les affectations intermédiaires, les sujets d'âge moyen.

La différence entre l'âge de 50 ans et l'àge actuel des massifs étant, par exemple, de 30 ans, 'la durée de la rérolution préparatoire serait donc de 30 ans. On diviserait cette révolution en autant de parties aliquotes qu'il y aurait d'unités dans le quotient du chiffre de ladite révolution par celui de la périodicité des coupes préparatoires, et chacune des affectations normales serait partagée en autant de coupes qu'il y aurait d'années dans une des subdivisions de la révolution; ou bien, si l'on jugeait avantageux de ne pas multiplier ainsi les coupes a fairé chaque année, on grouperait les affectations deux par deux ou trois par trois. Chaque groupe ne formerait qu'une série qui comprendrait naturellement autant de coupes qu'il y aurait d'années dans une des subdivisions précitées, à moins qu'on ne rendît ces coupes biennales, ce qui pourrait encore se faire pour des raisons d'économie ou de simplification de service. Au reste, l'établissement du règle- 
ment général des exploitations, pendant la révolution préparatoire, rentrerait exactement dans le cas prévu à la page 331 .

Après la révolution préparatoire, la forêt dont le sous-bois le plus âgé aura 50 ans et sera surmonté de nombreuses réserves, et dont le sous-bois. le plus jeune, âgé de 30 ans, contiendra sans doute beaucoup de brins de semence, la forêt ne se distinguera pas des autres futaies irrégulières. On en réglera: l'exploitation comme on le fait pour ces dernières, en lui appliquant une révolution principale d'autant plus longue que les jeunes massifs, ou plutôt que les massifs de la dernière affectation, pourront durer plus longtemps. Si cette révolution ne s'écarte pas trop de celle que l'on aurait adoptée comme normale, on la regardera comme définitive. Si elle s'en écarte trop, on prendra les dispositions nécessaires pour que les affectations normales ne puissent jamais être perdues de vue.

\section{$\$ 2$.}

- Règlement général des exploitations, d'un taillis composé complet et régulier à convertir, lorsqu'il n'y a pas de révolution préparatoire, et que la conversion est immédiate.

La méthode de transformation exposée dans l'article précédent, est celle que l'on a longtemps suivie à l'exclusion de toute autre. C'est la plus simple, et 
je le dirai dès à présent, la plus rationnelle, la plus recommandable au point de vue cultural; puisque c'est celle qui permet le mieux d'utiliser les ressources du peuplement pour la régénération naturelle; mais elle se signale, au point de vue économique, par deux inconvénients majeurs : d'abord, elle a pour effet d'altérer profondément le rapport soutenu. Par: le fait de la conversion immédiate, les coupes de taillis étant remplacées par de simples nettoienrents ou éclaircies et des extractions prudentes de vieux arbres, il est clair que les produits annuels sont trèsfortement diminués. Ensuite, elle retarde le début de la première révolution principale, et elle rend presque impossible l'adoption, pour cette première révolution, d'une durée égale à celle de la révolution normale.

Le premier de ces inconvénients, l'altération profonde du rappoit soulenu, a été longtemps le principal motif qui s'est opposé à la transformation de nos taillis sous futaie en futaies pleines, et aujourd'hui encore celte altération est l'objet d'une préoccupation très-vive. Aussi a-t-on cherchẻ et cherche-t-on à l'atténuer, voudrait-on même empêcher qu'elle ne se produisît, chose pratiquement îrréalisable et même théoriquement inadmissible. Quels que soient les expé. dients auxquels on ait recours, quelle que soit la lenteur que l'on apporte dans l'opération, on ne saurait passer d'une certaine révolution à une révolution plus longue, sans se condamner à une diminution temporaire de revenu; cette diminution pourra être très-faible et de 
courte durée; elle n'en surviendra pas moins. Dans l'hypothèse la plus favorable, celle où la contenance de la coupe annuelle serait réduite d'une quantité assez petite, pour que l'on perdît moins sur l'étendue que l'on ne gagnerait sur l'accroissement, la diminution du revenu, renfermée dans les plus étroites limites imaginableś, porterait encore sur un an, et la transfor'mation, pour être complète, exigerait plusieurs siècles, en supposant qu'elle ne fùt pas contrariée par les exi. gences de la végétation. On poursuit donc vraiment une chimère, quand on prétend transformer les taillis en futaie, sans abaisser la production temporairement. Cette diminution sera d'autant moins sensible que le temps consacré à la transformation sera plus long; mais comme ce temps ne peut pas être infini, la diminution ne saurait jamais être réduite à zéro. Quoi qu'il en soit, les seuls moyens d'atténuer le sacrifice qu'entraine la substitution du mode d'exploitation en fulaie à celui du taillis, consistent dans la suppression plus ou moins complète des coupes prẻparatoires, et dans la continuation plus ou moins longue des coupes de taillis sur certaines parties de la forèt. Nous ll'avons pas à discuter en ce moment l'emploi de ce dernier moyen, puisque nous avons supposé que l'on voulait convertir immédiatement la forèt sur toute son étendue; et quant au premier moyen, la suppression des coupes préparatoires, il · est évident qu'il ne saurait être appliqué qu'à la première affectation; car si la conversion de toute la forêt peut avoir lieu immédiatement, la régéné- 
ration ne peut assurément en être que graduelle.

La suppression des coupes préparatoires, dans le cas d'une conversion immédiate générale, équivaut donc à la suppression de la révolution préparatoire. On eutreprend sans délai les coupes de régénération. On soumet la forêt sans transition à une rérolution principale, et, comme on allonge la distance possible entre l'époque de la régénération de la première affectation et l'époque de la régénération de la dernière, d'un temps égal à la durée de la révolution préparatoire, on est moins gêné pour adopter une révolution principale qui soit en harmonie avec l'exploitabilité des essences. En outre, et c'est le but principal que l'on a en vue, en coupant à blanc étoc les peuplements de la première affectation, au lieu de se borner à les éclaircir, on se procure une augmentation de produits; mais tous ces avantages sont quelquefois achetés chèrement par les difficultés que la suppression de la révolution préparatoire entraîne pour la régénération naturelle, difficultés d'autant plus grandes que les taillis de la première affectation sont plus jeunes. Si, même avec une révolution préparatoire, il est désirable que les taillis les plus vieux soient colloqués dans la première affectation, à plus forte raison est-ce à souhaiter quand on renonce aux coupes préparatoires. Autrement, on rend non-seulement, comme je viens de le dire, la régérération beaucoup plus pénible et par conséquent plus coûteuse, mais on se condamne encore à des pertes d'accroissement. Je n'insiste pas en ce 
moment sur ces deux points, parce que j'aurai à les traiter in extenso plus tard. Je me borne à ajouter que la suppression de la révolution préparatoire ne me paraît toléráble, à la rigueur, que lorsque les taillis de la première' affectation sont assez âgés pour contribuer à la régénération, sinon par leurs semences, au moins par leur abri.

Cette suppression étant admise d'ailleur's, le règlement général des exploitations ne soulève aucune question qui n'ait déjà été prévue et résolue.

\section{$\$ 3$.}

Règlement général des exploitations d'un taillis composé complet et régulier à convertir, lorsqu'il u'y a pas de révolution préparatoire, et que la conversion est graduelle.

I. - Exposé des méthodes applicables. - La suppresșion de la révolution préparatoire atténue donc la diminution de produits inhérente à. la conversion, et.permet d'adopter, pour la révolution principale, une durée quii se rapproche davantage de la durée normale. Toutefois son effet, surtout sous le premier rapport, n'a point paru assez sensible, et l'on s'est demandé, si l'on ne pourrait point recourir à des moyens plus énergiques, soit pour augmenter les produits pendant la transformation, soit pour rendre possible l'adoption immédiate d'une révolution principale normale? La réponse étail facile : On 
avait supprimé les coupes préparatoires pour la première affectation, il n'y avait qu'à les supprimer aussi pour les autres.

Si on les supprime effectivement, alors il y a nécessité de continuer les coupes de taillis dans toules les affectations, sauf la première. La conversion n'est plus immédiate sur loute l'étendue de la forêt; elle est graduelle, et rien ne s'oppose à ce que l'on adopte la révolution principale normale. Pendant qu'on régémère la première affezlation dans le cours de la période normale correspondante, on continue le taillis dans les autres affectations jusqu'à ce que leur tour de régénération en futaie soit arrivé. Quant aux produits, si on ne les considère que dans leur 'ensemble, non pas pour une affectation plutôt que pour l'autre,' non pas par année, mais par période, ils seront nécessairement un peu plus forts dans la première période qu'ils ne l'auront été auparavant dans le même espace de temps; càr, sur' toute l'étendue de la première affectation, au lieu de se borner à receper les taillis sous la réserve d'un nombre d'arbres plus ou moins grand, on aura tout à coulper. La diminution de la production périodique ne se manifestera qu'à partir de la deuxième période, parce qu'alors, dans la première affectation, à la place des anciennes coupes de taillis, il n'y aura à faire que des éclaircies; et cette diminution ira en augmentant, de période en période, pendant la première 'révolution, jusqu'à ce que les bois précédemment régénérés soient assez àgés pour que l'on en 
obtienne, par de simples éclaircies, autant de produits qu'on en obtenait des coupes de taillis.

Ces résultats se comprennent facilement. Je vais cependant les mettre mieux en évidence, et montrer comment on peut les assurer par le roulement général des exploitations, quand on se résigne d'ailleurs à sacrifier les règles d'assiette, et qu'on ne tient pas à avoir pendant la période une suite non interrompue, tout à la fois, et pour les coupes de régénération et pour celles de taillis.

En effet, quelle que soit la composition des affectations normales; dès que le nombre des coupes du taillis à convertir est divisible par le nombre de ces affectations, - et il n'y a pas d'empêchement à ce qu'il le soit, - toutes les hypothèses de distribution des coupes de différents âges entre les affectations, peuvent se ramener à trois types auxquels je donnerai des chiffres pour qu'ils soient plus saisissants et, pour cela, je supposerai que le nombre des coupes de taillis est de 36 ainsi que le nombre des années de la période, et celui des affectations de 4. Chaque affectation contiendra dès lors 9 coupes et il se pourra :

1. Que les 9 coupes les plus êgées soient dans la première affectation, les coupes de 19 à 27 ans dans la deuxième, et les coupes les moins âgées dans la dernière;

$2^{\circ}$ Que cet ordre étant renversé, les 9 coupes les moins âgées soient dans la première affectation, et les 9 coupes les plus âgées dans la dernière; 
3. Que dans chaque affectation, il y ait des coupes dont les âges ne se suivent pas régulièrement.

Dans le premier cas, l'augmentation des produits aura lieu au commencement de la première période dans la première affectation, dont les neuf coupes seront exploitées à blanc étoc et régénérées. Après ces neuf coupes, les produits annuels, qui seront fournis par les autres affectations, reviendront au chiffre où ils étaient auparavant, pour se maintenir ainsi pendant 27 ans, c'est-à-dire jusqqu'à la deuxième période. A cette époque, il n'y aura que des éclaircies à faire, pendant 9 ans, dans les coupes régénérées de la première affectation dont les bois les plus vieux n'auront que 36 ans et, par conséquent, la production baissera plus qu'elle n'aura haussé au début de la première période; puis, on entreprendia la régénération des coupes âgées de 28 à 36 ans de la deuxième affectation et, pendant 9 ans, les produits se relèveront de nouveau. A partir de la dix-huitième année de la deuxième période, et jusqu'à la première année de la troisième, les produits fournis par les coupes de taillis dans la troisième et la quatrième affectation seront ce qu'ils étaient avant le commencement de la conversion; mais désormais il n'y aura que des éclaircies à pratiquer dans les deux premières affectations et il s'ensuivra une forte baisse dans les produits, baisse qui, en moyenne, sera plus prononcée que la précédente, à moins que les éclaircies à faire dans la première affectation ne soient aussi productives que 
l'étaient les coupes de taillis, chose improbable. Cette baisse ne cessera que lorsqu'on pourra entreprendre la régénération des coupes de la troisième affectation, c'est-à-dire à la dix-huitième année de la période correspondante. Après avoir régénéré ces coupes, et pendant les dernières années de la période, on coupera les taillis devenus exploitables de la quatrième affectation; après quoi, il n'y aura plus de coupes de taillis à faire, mais simplement des éclairciès, jusqu'à la vingt-huitième année de la dernière période, et il est possible que parmi ces éclaircies il y en ait, celles de la première affectation par exemple, 'qui fourniront autant de produits qu'en fournissaient les coupes de taillis; de sorte que, pendant la dernière - période, les produits pourront ne pas être moins élevés qu'ils l'auront été pendant la période précédente. $A$ la fin de la quatrième période, les coupes de la quatrième affectation seront régénérées, la transformation sera complète et la hausse croissante des produits se manifestera de plus en plus.

Dans le deuxième cas, la première affectation n'étant régénérée qu'à la fin de la période correspondante, c'est à la fin de cette période qu'a lieú l'augmentation des produits, et la baisse ne survient qu'à la fin de la deuxième période, au lieu de se faire sentir all commencement.

Dans le troisième cas, les variations dans les produits ne suivent plus aucune marche régulière: selon la disposition des peuplements; d'après leur 
âge, la hausse peut avoir lieu, soit au commencement, soit à la fin, soit au milieu d'une période; mais la baisse n'en est pas moins inévitable; et, comme dans les deux autres cas, elle l'emporte de plus en plus sur la hausse, jusqu'à ce que les éclaircies soient devenues aussi productives que l'étaient les coupes de taillis. Voilà la loi qui domine toutes les autres.

Lorsque la bonne formation des affectations normales se concilie avec la collocation des taillis les plus vieux dans la première affectation, des plus jeunes dans la dernière, de ceux d'âge moyen dans les intermédiaires, on ne doit pas hésiter à adopter, pour la marche des coupes, le règlement général que j'ai exposé premièrement. Outre l'avantage de retarder l'époque de la baisse des produits, il a celui, beaucoup plus sérieux, de permettre que la durée de la période ne soit pas égale à la révolution des taillis, sans qu'il en résulte une perte d'accroissement. Ainsi, les taillis devant être coupés à 36 ans, rien ne s'opposerait, pourvu toutefois que le nombre des coupes de taillis fût toujour's divisible par celui des affectations où ces coupes devraient être assises, rien ne s'opposerait à ce que la période, dans le règlement général provisoire, fût fixée, comme dans le règlement définitif, à 24 ans ou à tout autre chiflre qui s'accorderait mieux avec les exigences de la régénération en futaie. C'est là ce qui est impossible quand les coupes sont distribuées entre les affectations comme on l'a indiqué dans les deux derniers cas. II 
est-obligatoire alors, si l'on veut tout à la fois ne couper les taillis qu'à l'âge d'exploitabilité, et régénérer chaque affectation en futaie dans la période correspondante, que la durée de cette période soit au moins aussi longue que l'ancienne révolution du taillis. Il est visible, par exemple, que si cette durée était de 24 ans seulement, tandis que la révolution du taillis serait de 36 ans, la première affectation ne pourrait, tout entière, être régénérée dans la première période, que si elle contenait au début de la conversion des taillis âgés de plus de 12 ans.

Un autre défaut des modèles de règlements qui précèdent, et ce défaut existe pour le premier modèle comme pour les autres, c'est la brusquerie dans les mouvements de hausse et de baisse, brusquerie qui devient très-accentuée quand on considère à part les produits de chaque affectation, et que l'on sépare ceux des coupes de régénération de ceux des coupes de taillis. On a eu recours à diverses combinaisons pour y obvier. La meilleure est sans contredit celle qui consiste à disposer les peuplements dans les affectations, de manière que chacune forme une série complète de coupes de taillis se suivant de proche en proche et d'âges gradués, depuis l'âge d'un an jusqu'à l'âge d'exploitabilité du taillis. Mais cela ne se peut guère que lorsqu'on a autant de séries de taillis à convertir que d'affectations de futaie à constituer. Toutefois, quand la chose est faisable, la marche des coupes, soit de régénération, soit de taillis, est parfaitement régulière: Pendant qu'on 
régénère par coupes successives, non interrompues et égalementproductives, la première affectation; on continue les coupes de taillis dans les autres; et, durant toute la première période, on a une augmentation de produits équivalente, chaque année, à la différence entre le rendement d'une coupe de taillis et celui d'une coupe à blanc étoc.

L'amoindrissement des produils commence au début de la deuxième période et se traduil, chaque année, par la différence entre le rendement d'une coupe de taillis et celui d'une coupe d'éclaircie; mais l'augmentation, pendant la première période, et l'amoindrissement, pendant la dernière, se répartissant également entre toutes les années de chaque période, on évite ces alternatives brusques de hausse et de baisse qu'entrainent les autres modes de transformation, et c'est un grand avantage. Malheureusement, on ne peut non plus se le procurer qu'à la condition d'adopter pour la période une durée égale à celle de la révolution du taillis, ce qui est regrettable, attendu que la durée des périodes ne doit, en bonne règle, être subordonnée qu'aux exigences de la régénération en futaie. D'ailleurs, je le répète, la possibilité de composer les affectations de telle sorte, que chacune d'elles forme une série de coupes de taillis, se présente très-rarement. Dans la plupart des cas, la gradation régulière dans les mouvements de la production ne peut être obtenue que lorsqu'on se résigne à des sacrifices d'accroissement, c'est-à-dire à ne pas couper le taillis à l'àge auquel on l'avait. 
coupé jusqu'alors; et ce qu'on gagne au point de vue $\mathrm{du}$ rapport soutenu, on le perd et au delà au point de vue de la quantité des produits.

Ainsi, quoi que l'on fasse, la conversion, même graduelle, d'un taillis sous futaie en futaie pleine, amène une diminution de produits, à partir de la deuxième période de la révolution principale. Cette diminution peut être régularisée ou retardée, rejetée du commencement de la période, au milieu ou à la fin. On ne saulait dans aucun cas l'éviter, à moins qu'en même temps que l'on convertit la première affectation en futaie, on ne Iransforme les autres en taillis simples en y exploitant les vieilles réserves. On comprend que par ce moyen, dont je montrerai plus tard tout le danger, on arrive à combler dans une large mesure, le déficit causé dans les produits par. la substitution des coupes de nettoiement et d'éclaircie aux coupes de taillis. On comprend que l'on puisse même, par l'abatage de ces vieilles réserves, rendre encore plus forte l'augmentation de produits qui est la suite, dans la première période de la révolution principale, de la substitution des coupes de régénération à celles de taillis composé; et là est tout le secret de ces merveilleux projets de transformation sur lesquẹls on s'est appuyé, pour soutenir que la conversion des taillis composés en futaie peut avoir lieu, sans occasionner temporairement une baisse dans la production de la forêt. Cependant, qu'on ne s'y trompe pas: même quatid on a recours à l'explo:tation des vieux arbres, la baisse des produits n'est 
que retardée, et l'on ne se procure plus de rendement dans les premières périodes que sous peine d'en avnir moins dans lés dernières.

En tout cas, et qu'on adopte l'un ou l'autre des modes de transformation ci-dessus décrits, le règlement général des exploitations par période se dresse toujoul's conformément au cadre donné p. 318 et 319 pour la marche provisoire des coupes, la révolution principale étant définitive; il y a place dans ce cadre pour toutes les coupes à faire de quelque nature qu'elles soient.

II. - Observations sur les mélhodes de conversion exposées dans l'arlicle précédent. - Les dispositions qui ont été imaginćes, pour éviter les inconvénients de l'ancienne mélhode de transformation des laillis composés en futaies pleines, se caractérisent toutes par ces trois circonstances : suppression des coupes prépara-. toires; continuation des coupes de taillis dans les différentes affectations jusqu'à l'époque où elles arriveront en tour de régénération en futaie; extraction des vieilles réserves. Nous en connaissons la valeur économique, no:ss allons en apprérier le mérite cultural :

Suppression des conpes préparaloires. La suppression des coupes préparatoires a pour effet d'amoindrir, jusqu'à les annihiler' quelquefois, les chances du repeuplement naturel. Elle est donc conIraire à l'un des principes fondamentaux de la culture forestière. Il est extrèmement important d'ailleurs, surtout lorsque cette culture se pralique sur de vastes 
étendues et réclame une nombreuse administration, que l'homme ne se charge pas à la légère des opérations que la nature peut faire elle-même. Les soins minutieux, les travaux de tout genre, que comporte" la gestion des forêts, seront toujours assez multipliés pour qu'on ne les augmente pas sans nécessité absolue. Or, pour transformer un taillis composé en futaie pleine, il n'est pas nécessaire de recourir aux repeuplements artificiels. Étant donné un taillis simple de chêne et de hêtre, âgé de 25 à 30 ans, il est certain qu'en le laissant croître jusqu'à l'àge où il pourra donner des semences fertiles, jusqu'à 70 ans par exemple, on le mettra dans de bonnes conditions pour le repeuplement naturel; tandis que si on le coupe aujourd'hui, on ne pourra se dispenser de repeupler le sol artificiellement. Cette obligation du repeuplement artificiel s'appliquera sans doute, toutes proportions gardées, à une moins grande étendue, si au lieu d'avoir affaire à un taillis simple, on a affaire à un taillis composé; mais il est facile. de se rendre compte de l'économie que les réserves, par leurs semences, permettront de réaliser dans les repeuplements à faire de main d'homme : nous n'avons pour cela qu'à ouvrir le cours de culture de MM. Lorentz et Parade à l'article concernant le balivage normal : nous y verrons que le couvert des réserves, au moment de la coupe du taillis, peut comprendre 1/3 de l'aire de la coupe. Dans les circonstances les plus favorables, les travaux de repeuplement qu'entraînerait la suppression des coupes 
préparaloires, s'étendraient donc encore aux $2 / 3$ de la surface d'une coupe. Quand on peut éviter des travaux aussi considérables, on doit le faire; d'autant qu'il n'y aurait rien d'étonnant à ce qu'un brin de semence provenant d'un semis artificiel, fùt moins vivace qu'un brin provenant d'un semis naturel. N'y a-t-il pas dans le travail de la nature, une force mystérieuse que la science de l'homme n'a point pénétrée et ne saurait mettre dans ses œuvres ? Quant à moi, je penche, je l'avoue, pour l'affirmative, el, de même que toutes ces précocités artificielles si merveilleuses obtenues pour la viande, pour les fruits, ne fournissent pas des aliments aussi substantiels que ceux qu'on retire des productions qui ne sont pas forcées et dont la marche reste conforme aux lois de la nature; de même, j'incline-ì le penser, un plant, élevé en pépinière, ne vaut pas celui qui est venu spontanément en plein bois, et des graines, semées dans une autre localité que celle où elles ont été réco!tées, ne sauraient produire des sujets aussi robustes que ceux qui proviennent de graines récoltées et semées dans la même région. En outre, quelle que soit la préparation que l'homme donne au terrain, quelle que soit l'abondance avec laquelle il y jette les semences, cette préparation ne vaut pas l'humus, et cette abondance n'est que de la parcimonie à côté de la prodigalité de la providence.

La suppression des coupes préparatoires tendant en un mot à substituer l'homme à la nature, dans un travail qu'elle fait mieux que lui, n'est donc pas 
une bonne chose; ces coupes d'ailleur's sont trèsutiles :

$1^{\circ}$ Afin que le sol, souvent appauvripar un mode d'exploitation qui empêche l'humus de s'y accumuler, ait le temps de se refaire;

$2^{\circ}$ Parce qu'il est de la plus grande importance pour les repeuplements, soit naturels, soit artificiels, de n'exploiter les taillis qu'ils sont destinés à remplacer, qu'à l'àge où l'on n'a plus à craindre leur reproduction par la souche; cette reproduction est en effet, aucun forestier praticien ne l'ignore, une des entraves qui nuisent le plus aux jeunes plants et dont il est le plus difficile de les débarrasser;

$3^{\circ}$ Parce que, dans le cas même où on se décide par des motifs quelconques à renoncer au repeuplement naturel, il est économique, pour le repeuplement artificiel, de se procurer un abri, abri qui ne peut ètre assuré suffisamment que par des sujets dont la tèle est bien for'mée.

Ces observations ne soulèvent aucune objection pour l'affectation qui ne doit plus être coupée en taillis, qui contient ordinairement les bois les plus vieux, et dans laquelle il n'est plus temps, il n'est plus possible de songer à améliorer le peuplement en brins de franc-pied par de fréquents nettoiements et des plantations faites après la coupe; mais pour les autres affectations elles paraîtront, je le prévois, moins fondées. On fera sans doute remarquer que, si l'on prend soin de dégager tous les brins de semence qui se révéleront après la coupe du taillis, et de faire quelques 
plantations, on se procurera les moyens, à la coupe suivante, de constituer la jeune réserve avec des sujets de franc-pied et par conséquent d'avenir; et que, si l'on porte en outre celte réserve à 150 baliveaux et même au delà, il ne faudra pas plus de deux révolutions de taillis pour réunir les éléments d'une régénération naturelle complète. A cela je répondrai que les coupes dites de taillis, dans lesquelles on conserve autant de baliveaux, ne sont en réalité que des coupes préparatoires, sous une forme particulière, et qu'on a tort de leur donner une qualification qui n'est plus justifiée, qui est même tout à fait démentie par le balivage serré dont elles sont l'objet, puisque ce balivage a pour conséquence inévitable de nuire de plus en plus au développement des rejets de souche. Je suis d'ailleurs persuadé qu'on se fait illusion, si l'on croit qu'il est facile, par des nettoiements répétés, de dégager les brins de semence qui apparaissent souvent en abondance, j'en conviens, immédiatement après l'abalage des taillis. Ce dégagement n'est point aussi commode qu'on le pense, car il faut y revenir fréquemment et quelquefois tous les ans. Enfin, si les taillis composés présentent l'inconvénient de ne pouvoir former que des futaies irrégulières dans lesquelles, au milieu de jeunes massifs sur souche, homogènes par places, sont disséminés des arbres d'âges et de grosseurs très-différentes, je ne m'explique pas que l'on veuille gratuitement aggraver cet inconvénient, en mettant dans le: jeunes bois les inégalités qui sont dans lez vieux, 
et en ajoutant ainsi d'autres étages à ceux qui existent déjà. Il me semble que de tels procédés s'écartent des principes de la culture, soit en ce qui concerne le taillis, soit en ce qui concerne la futaie, et que, fâcheux pour la production du taillis, ils le sont également pour la formation de la futaie.

Continuation des coupes de taillis. - S'il est vrai, comme tous les auteurs s'accordent à lo dire, que le mode d'exploitation en taillis soit compromettant pour la conservation de la qualité du sol et pour celle des essences les plus précieuses, surtout pour le chêne, toute combinaison tendant à ajourner la suppression des coupes faites d'après ce mode, doit être considérée comme fort regrettable; mais si l'on renonce à cet ajournement, il est clair qu'on rend d'abord la transformation très-longue, puisqu'on n'arrivera à la compléter qu'après plusieurs révolutions transitoires de futaie; et qu'on se condamne ensuite à une diminution immédiate et très-sensible dans la production annuelle. La continuation des coupes de taillis, en maintenant jeunes certaines affectations; tandis qu'on régénère ou qu'on prépare les autres, avance évidemment l'époque de la constitution définitive des classes d'âge, et pare en même temps à une diminution trop brusque des produits.

On peut donc admettre cet expédient, pourvu que d'ailleurs le sol soit bon et le taillis vivace; mais, même alors, il convient d'en user avec modération, et d'y renoncer dès que le peuplement, qu'il aura servi à rajeunir, paraîtra susceptible de rester 
sur pied jusqu'au moment où viendra son tour de régénération en futaie. En outre, bien que cette continuation des coupes de taillis ait surtout pour but d'éviter une trop forte baisse dans la produclion, ce n'est point une raison pour que le taillis soit exploité avant l'àge où il fournit tout à la fois et les

- meilleurs produits et le plus de ressources pour un balivage satisfaisant; de sorte que si les plus vieux peuplements avaient été classés dans les affectations qu'il ne serait point nécessaire de rajeunir, il vaudrait mieux interrompre les coupes de taillis au début de la transformation, que d'abattre des bois trop jeunes. Enfin, ce que j'ai dit plus liaut, à propos des coupes de taillis dans lesquelles on a proposé de forcer le balivage au delà des limites indiquées par les exigences de la reproduction des souches, est encore applicable ici. Les coupes ainsi faites ne sont plus des coupes de taillis : ce sont des coupes d'amélioration ou des coupes préparatoires; tandis que les méthodes de transformation que nous étudions en ce moment, sont basées sur la continuation des coupes de taillis traditionnelles et dans lesquelles, par conséquent, le taillis ne doit pas être sacrifié complétement à la futaie.

Extraction des vieilles réserves. - Assurément, c'est une règle très-raisonnable en théorie que celle qui autorise à enlever les vieux arbres dans les affectations, conservées temporairement en taillis, toutes les fois qu'ils paraitraient hors d'état de rester sur pied, sans dépérir, jusqu'à l'époque où 
ces affectations arriveront en tour de régénération en futaie; mais cette règle est pleine de dangers : D'abord, il ne faut point se dissimuler que tant qu'un peuplement continue à être traité en taillis, sa conversion reste douleuse, et que ce n'est point parce qu'elle figure sur le papier qu'on doit la regarder comme assurée. Les flots et les destins sont changeants; les plans d'exploitation le sont aussi, et il est prudent de prévoir le cas où la conversion, bien qu'ordonnée par un décret, ne serait point exéculée; or, dans ce cas, si l'on avait dépouillé cette affectation de toutes les vieilles réserves; au lieu d'un taillis composé susceptible de fournir des produits à l'industrie, on n'aurait plus qu'un taillis simple. Ensuite, on doit se rappelel que c'est surtout en utilisant les semences qui tombent en abondance des vieux arbres, qu'on peut maintenir les taillis dans un bon état de consistance et de vitalité. Je pense donc qu'il convient de ménager beaucoup ces vieux arbres, tout en reconnaissant qu'on peut en extraire d'autant plus que l'affectation, dont ils font partie, est moins près d'être régénérée en futaie. 


\section{$\$ 4$.}

Règlement général des exploitations d'un taillis composé régulier et complct à conver.ir, lorsqu'on adopte une révolution préparatoire et que la conversion est graduelle.

Si les observations contenues dans le paragraphe précédent sont justes, il en découle qu'on doit poser comme principes fondamentaux de la transformation d'un taillis sous futaie complet et régulier, en futaie pleine :

$1^{\circ}$ La préparation des peuplements pendant assez longtemps, pour que le taillis puisse concourir au réenșemencement naturel, et soit hors d'ćtat de rejeter abondamment par la souche;

$2^{\circ}$ La limitation de l'ancien mode d'exploitation en taillis, aux peuplements qui ne seraient pas susceptibles de rester sur pied, sans dépérir, jusqu'à l'époque fixée pour leur régénération en futaie dans la révolution principale, et qu'on ne pourrait avant cette époque remplacer par des brins de semence, sans occasionner de trop fortes pertes d'accroissement;

$3^{\circ}$ L'exploitation du taillis à un âge qui ne soit pas inférieur à celui qui avait été adopté jusqu'alors, et qui permette dans tous les cas de trouver dans cetaillis les éléments d'un balivage satisfaisant;

$4^{\circ}$ Le maintien de la futaie sur taillis dans un 
état normal, c'est-à-dire dans un étal de richesse aussi grand que le comportent les exigences de la reproduction et de la végétation du sous-bois.

Le premier de ces principes implique l'adoption d'une révolution préparatoire dont la durée sera d'autant plus longue, que l'âge des bois dans les affectations à régénérer les premières, sera moins avancé : il n'est pas indispensable, mais il peut-être utile, comme mesure d'ordre, que cette révolution soit un multiple de la période de la révolution principale.

En ce qui concerne le deuxième principe, il semblerait au premier abord que s"il y a une affectation que l'on puisse à coup sûr, sans s'écarter de ce prineipe, continuer d'exploiter en taillis, c'est bien la dernière; cependant, en y réfléchissant, on voit qu'il y a un moyen de la rajeunir qui est préférable: e'est de la régénórer en futaie en même temps que la première affectation', et après l'avoir préparée comme elle pendant la révolution préparatoire. A l'époque où la dernière aflectation arrivera de nouveau en tour de régénération, e'est-àdire à la fin de la première révolution principale, elle n'aura pas encore atteint, il est vrai, l'âge correspondant à la durée de cette révolution; mais il est présumable que, malgré la perte qui s'ensuivra, les produits seront encore plus considérables que ceux qu'on obtiendrait des coupes de taillis. Ainsi je suis d'avis que ces coupes de taillis doivent être arrêtées dans la première et dans la dernière affectation d'abord; 
ensuite, dans la deuxième, et j'irai mễme jusqu'à proposer d'y renoncer aussi dans la troisième, attendu qu'il est sage de ne pas marchander les sacrifices en vue d'un très-grand bien.

Pour se conformer at troisième principe, sans se 'meltre dans la nécessité d'interrompre les coupes de taillis dès le début de la transformation, il faut que la troisième affectation en contienne d'exploitables, et je n'y vois pas d'inconvénient, lorsque cela peut se faire sans "contrarier les règles d'assiette.

Quant au quatrième principe, il est toujours applicable, quelles que soient les combinaisons adoptées, et je me borne à dire que la conservation des vieilles réserves est d'autant plus utile dans une affectation, que celle-ci est plıs près de son tour de régénération.

L'application de ces quatre principes étant ainsi précisée, la formation du règlement général d'exploitation, soit pendant la révolution préparatoire, soit pendant la première révolution principale, n'exige plus aucun éclaircissement et je prie encore le lecteur de se reporter pour le cadre à prendre aux pages 318 et 323 .

La méthode que je recommande ${ }^{1}$ est donc une transaction entre l'ancien système qui arrêtait immédiatement les coupes de taillis sur toute l'étendue de

1. Ce système est exposé avec beaucoup de clarté dans un article des Annales forestières (tome III), article qui est le point de départ de toutes les conceptions qui onl été basées depuis sur la continualion des coupes de taillis. 
la forêt et le nouveau système également radical, en sens contraire, qui ne les arrête que successivement, lorsque les peuplements arrivent en tour de régénération dans la révólution principale; mais elle se rapproche beaucoup plus du premier que du second, puisqu'elle lend, comme le premier, à utiliser autant que possible les peuplements existants, pour la régénération naturelle, tandis que le second système fai peu de cas des ressources qu'on en pourrait tirer sous ce rapport ${ }^{1}$.

Il est incontestable qu'au point de vue cultural, la substitution des repeuplements artificiels aux repeuplements naturels est regrettable. Les exigences du rapport soutenu et l'avantage d'une création plus rapide des classes d'àge qui constituent une forêt normale, ne la justifieraient pas suffisamment à mes yeux; et d'ailleurs, puisque la méthode mixte de transformation que nous avons examinée en dernier, tient compte dans la mesure du possible, et de ces exigences, et de cet avantage, il ne saurait y avoir d'hésitation à l'adopter. Comme celles qui ont été décrites auparavant, cette dernière méthode repose sur la supposition que le taillis composé à converlir, contient non-seulement de nombreuses réserves, mais encore un sous-bo:s vivace, riche en essences de bonne qualité, complet, susceptible de coopérer tôt

1. M. Puton, professeur à l'École forestière de Nancy, a fort bien expliqué et justifié cette méthode, qu'il appelle la méthode mixte dans son livre sur l'aménagement des forêts. (Rothschild, éditeur) 
ou tard, dans une forte proportion, à la régénération de la forèt par les semences. C'est cetle dernière circonstance qui motive surtout les coupes préparatoires et la cessation la plus prompte possible des coupes de taillis. Lorsqu'elle fait défaut, les coupes préparatoires n'ont plus autant de raison d'être, et ce n'est plus dans le mème but que l'on supprine les coupes de taillis. Or, tous nos taillis sous futaie ne se trouvent pas dans des conditions salisfaisantes au double point de vue de la consistance de la réserve et de celle du sous-bois. L'application des combinaisons dont j'ai décrit le mécanisme rencontre donc souvent des exceplions. Je vais en examiner quelques-unes :

\section{$\$ 5$.}

Rèollement général des exp!oitations lorsque les taillis à convertir sont irréguliers et incomplets.

J'appelle irrégulier's les taillis composés qui s'éloignent de l'état normal, soit parce que les réserves en chêne et en hêtres de toute catégorie, n'y sont point également réparties, soit parce que le sous-bois y est principalement-formé d'essences secondaires. Ces taillis peuvent ètre complets ou incomplets : complets, quand ils ne présentent ni vides, ni clairières, ni peuplements ruinés; incomplets, quand ils en présentent.

Supposons que l'on ait à convertir un taillis com- 
posé complet, mais dans lequel il y a les anomalies suivantes :

Les réserves en essences d'élite, assez nombreuses dans quelques parcelles, sont rares dans les autres. Le taillis dont le charme et les bois blancs forment les essences presque exclusives, surtout dans les coupes les plus âgées, sont garnies dans les jeunes, et en assez grande quantité, de brins de hêtre et de chène qu'on pourrait encore sauver, en les dégageant des rejets qui les dominent. Le sol est d'ailleurs partout de bonne qualité.

En présence de cette situation, c'est surtout d'après l'àge et le nombre des rẹ́serves que contiennent les parcelles que l'on se sera guidé, toute satisfaction ayant été d'ailleurs donnée aux règles d'assiette, pour composer les affectations, et, par conséquent, on aura mis dans la première affectation les parties les plus riches en vieux arbres. Les jeunes coupes où il y a beaucoup de brins de hêtre et de chêne auront été placées autant que possible dans la dernière, et les autres coupes formeront les affectations intermédiaires.

Les affectations étant ainsi constituées, on adoptera une révolution préparatoire juste assez longue pour qu'à son expiration l'on puisse, avec les taillis de charme et de bois blancs de la première affectation, faire de bonnes coupes d'abri; et tandis qu'on se bornera jusque-là à exécuter dans cette affectation des coupes préparatoires, on s'efforcera de dégager, au moyen de nettoiements fréquents, les brins de 
chêne et de hêtre de la dernière, et on continuera de couper en taillis les autres affectalions en commençant par la deuxième. Il n'y aurait en effet aucun avantage pour la régénération naturelle à soumeltre immédiatement les affectations intermédiaires à des coupes préparatoires, puisque l'on n'altend rien pour le repeuplement, des semences que pourraient donner les taillis qui les composent; on aura le temps de préparer la deuxième affectation pendant qu'on régénérera la première.

Si les réserves étaient partout nombreuses, mais âgées, les autres conditions restant les mêmes, on ne mettrait encore en préparation que la première affectation; mais il faudrait répéter les nettoicments dans les autres et surtout dans les dernières très-fréquemment, et y conserver tous les brins d'essences d'élite, dùt-on entraver la reproduction des rejets, compromettre par conséquent la conservation du taillis, et se condamner à raccourcir la durće de la première révolution principale; l'essentiel serait d'utiliser les réserves avant leur complet dépérissement : ces réser'ves ne seraient abaltues que lorsquelles menaceraient de tomber de vétusté, ou lorsqu'elles surmonteraient de jeunes semis.

Si, au lieu d'un taillis exclusivement composé d'essences secondaires, on avait un taillis dans lequel ces essences seraient entremêlées de bois précieux dominants par place; alors il conviendrait, pendant qu'on préparerait la première affectation et qu'on dégagerait les jeunes brins dans la dernière, de préparer 
aussi la deuxième, et peut-être même la troisième, ce qui n'empêcherait pas quand on y rencontrerait des taches de bois blancs à l'état pur, de receper à blanc étoc ces bois blancs, toutes les fois qu'il ne pourraient rester sur pied jusqu'à l'époque oì l'affeclation dont ils feraient parlie arriverait en tour de régénération. Ce recepage, qui ne serait qu'un pis aller, n'autoriserait pas à dire qu'il faut continuer le taillis dans ces affectations-là.

Supposons maintenant que l'on ait à convertir un taillis sous futaie irrégulier et incomplet en ce sens, qu'indépendamment de la mauvaise qualité des essences sur beaucoup de points, il présente des lacunes, des vides, des peuplements sans avenir. Ici, de nombreuses mais vieilles réserves recouvrent des cépées de bois blancs clair-plantées; là, le taillis est assez serré et riche en bois durs, mais les souches sont usées, et les réserves font défaut; enfin, sur la plus grande étendue il n'y a que des vides, des clairières et des broussailles sur un sol appauvri.

$\mathrm{O}$ is voit tout de suite que la transformation d'une telle forêt n'exige pas de révolution préparatoire : il faut se servir sans délai des vieux arbres pour la régénération naturelle, se préoccuper d'utiliser les taillis de bois durs dans le même but, et denner le plus tôt possible au sol des parties ruinées, un couvert qui lui rende la fertilité qu'il a perdue. On assignera en conséquence le $n^{0} 1$ à l'affectation la plus riche en réserves, et on y prescrira immédiatement des coupes de régéneration avec enlèvement des bois 
blancs par extraction de souches. Les taillis de bois durs placés dans la deuxième et, s'il y a lieu, la troisième affectation, seront améliorés pendant la première période, par les moyens qu'on jugera les plus convenables; les vides, les clairières, les broussailles, rejetés dans les dernières affectations, seront les uns repeuplés, les autres complétées ou régénérées avec des essences peu exigeantes et choisies en vue plutôt d'améliorer le terrain que de se procurer des produits. Les coupes de taillis arrêtées partout, seront remplacées par des recepages qui marcheront d'autant plus vite, que les moyens d'action dont on disposera pour les repeuplements artificiels, seront plus énergiques; car les rapailles elles-mêmes ne devront être coupées, que lorsqu'on sera en mesure de les remplacer par un couvert plus utile.

Je pourrais faire beaucoup d'autres hypothèses qui motiveraient aussi des exceptions aux règles que j'ai posées, mais je ne le crois pas nécessaire. J'en ai dit assez pour montrer que dans la transformation d'un taillis irrégulier et incomplet en futaie pleine, les dispositions à prendre dépendent surtout du nombre, de l'âge, de l'état et de la répartition des réserves; que le sous-bois a généralement peu d'influence à ce sujet, et que son exploitation doit être subordonnée complétement aux exigences de l'amélioration du sol et à celle de la propagation des essences d'élite. On voit donc que le succès de l'opération repose beaucoup moins sur l'habileté des agents aménagistes, que sur celle des agents d'exécution. 
En résumé, dans les conversions des taillis composés, comme dans les aménagements des futaies irrégulières en futaies pleines, le but que l'on se propose étant de créer les classe d'âge indispensables, pour que l'on puisse retirer d'une forêt le rapport annuel le plus avantageux, il importe tout d'abord, dans un cas comme dans l'autre, d'assigner à ces classes d'âge la place qu'elles occuperont plus tard; puis, les affectations étant déterminées, on doit chercher à régler leur exploitation de manière à accélérer autant que faire se pourra la régénération de la première affectation, et à accumuler dans les autres affectations, pour l'époque à laquelle elles arriveront en tour d'exploitation principale, le plus d'éléments possible de régénération naturelle.

- Qu'on s'occupe en outre à retirer d'ici là le plus grand produit de ces affectations, rien de plus légitime, pourvu que le résultat essentiel à poursuivre ne soit pas compromis, et c'est dans cette condition que réside la difficulté de la transformation.

Les procédés de culture à appliquer aux affectations, en attendant que leur tour de régénération en futaie soit arrivé, sont uniformes dans les forêts régulières : il n'y a que des nettoiements ou des éclaircies à faire. Dans les taillis composés en conversion, surtout lorsque ces taillis sont irréguliers, ces procédés sont variables' et multiples : suivant les circonstances, on peut avoir à faire une coupe de taillis, un recepage, une substitution d'essence, un nettoiement, une éclaircie, une coupe préparatoire, une 
simple extraction de vieux arbres. Mais cetle différence entre le traitement d'une forêt régulière et celui d'un taillis sous futaie en conversion, quelles que soient la consistance et la composition de ce dernier, ne change rien aux dispositions essentielles du plan d'exploitation et à la forme des tableaux qui le résument. Ces tableaux, dont on a vu les modèles pages $31 \mathrm{~S}$ et 323 , peuvent servir à tous les cas. Toutes les fois qu'une affectation devra être exploitée systématiquement en taillis pendant une ou plusieurs périodes, on la fera figurer, sur. le tableau général, dans la colonne des coupes de taillis. Toutes les fois au contraire qu'elle ne devra être exploitée en taillis qu'exceptionnellement et incomplétement; que ce mode ne sera qu'un pis aller, un expédient auquel il serait possible et désirable qu'on ne fùt point obligé de recourir, on ne fera pas de distinction entre elle et les parcelles qui devront' être améliorées, et on l'inscrira dans la colonne des coupes d'amélioration, afin d'éviter de lui donner une destination qui pourrait être démentie par les circonstances. On se bornera à indiquer dans la colonne d'observations le traitement dont il y a probabilité qu'elle devra être l'objet. Rien' de plus simple, n'est-ce pas? mais cependant pour qu'il n'y eût : aucune confusion dans le tableau des exploitations et que le roulement des coupes de diverses nalures s'y reconnût, 's'y distinguât clairement, : il faudrait que la durée de la rotation pour, chaque catégorie de coupes, fût une partie aliquote de la période. Or il est difficile que cette condition puisse 
être réalisée pour toutes les coupes, et qu'une rotation ne soit pas à cheval sur deux périodes. L'important est que cette irrégularité n'existe pas pour les coupes les plus essentielles et surtout pour celles de taillis. Nous avons déjà reconnu q:e lorsque, cas trèsrare d'ailleurs, il était possible et il convenait de former une série de coupes de taillis par affectation, on ne pouvait guère se dispenser de donner à la période une durée au moins égale à la révolution du taillis. Lor'squ'on ne cherche point à former une série de coupes de taillis par affeclation, l'utilité d'avoir une correspondance complète entre la durée de la période et la périodicité des coupes de taillis est moindre; mais il convient alors, sous peine de n'éviter une confusion que pour tomber dans une autre, d'exploiter les coupes de taillis à un âge qui soit divisible par le nombre des affectations dans lesquelles ces coupes seront assises. Dans tous les cas, on se rappellera que la durée de la période normale ne doit être subordonnée qu'aux exigences de la régénération en futaie qui en sont la raison d'ètre, et qu'il y aurait de grands inconvénients à ce qu'on s'écartât de cette règle sans modération; de sorte que si le taillis devait être coupé à 36 ans, tandis que la période, eu égard aux exigences de la régénération, ne devrait embrasser que 30 ans, il serait tout à fait irrationnel, pour satisfaire à de pures et temporaires convenances de forme, de porter la période à 36 ans.

Ces principes trouvent leur application quand on dresse le tableau des exploitations à faire pendant la 
révolulion principale. En ce qui concerne le tableau des exploitations pendant la révolution préparatoire, les mêmes difficultés ne se rencontrent pas, puisqu'il n'y a à faire que des coupes par contenance et que la périodicité de ces coupes est la seule circonstance qu'il y ait à considérer, quand il y a lieu de partager la révolution en un cerlain nombre de subdivisions.

ARTICLE IV.

RËGLEMEXT SPËCIAL DES EXPLOITATIONS PEXDANT LAPREMIERE PÉRIODF, QUELLE QEE SOIT

LA MÉTHODE ADOPTEீE POCE LA TRAXSFORMATIOX.

Si le règlement général des explo:tations par période est plus ou moins compliqué et exige des explications particulières, suiyant l'état des peuplements et la rapidité que l'on veut imprimer à la conversion, il n'en est pas de même du règlement spécial qu'il est nécessaire de dresser pour les coupes à faire dans le cours de la première période, soit de la révolution préparatoire, soit de la révolution princi-. pale quand il n'y a pas de révolution préparatoire.

Le règlement pour les coupes de régénération doit être basé sur le volume, toutes les fois que le matériel existant dans l'aflectation à régénérer, est susceptible d'être cubé avec une exactitude suffisante; parce qu'alors il n'y a aucune différence à établir 
entre la forêt à transformer et une futaie irrégulière quelconque à aménager. Il est au contraire basé sur la contenance, lorsque ledit matériel se compose de rejets ou de brins de très-faibles dimensions dont on ne pourrait faire le cubage avec une suffisante exactitude, sans y consacrer beaucoup de temps et des soins minutieux. Dans ce dernier cas, d'ailleurs, la régénération devant avoir lieu en grande partie artificiellement, il est possible d'imprimer à cette régénération, sans la compromettre, une marche régulière de proche en proche; mais dans aucune circonstance, on ne doit combiner la possibilité par volume avec celle par contenance, car elles sont véritablement inconciliables. Si donc on adopte la possibilité par volume, alors il n'y a pas de distinction à faire entre les coupes de régénération : elles prennent chacune, chaque année, une part plus ou meins forte de la possibilité, suivant l'état des peuplements. Si au contraire on adopte la possibilité par contenance; comme elle n'est évidemment applicable qu'aux coupes d'ensemencement, les bois qui restent après ces coupes doivent être considérés comme étant en dehors de la possibilité, et c'est aux agents locaux qu'il appartient d'en proposer l'extraction, sans s'astreindre à aucune règle prévue d'avance, là où le besoin s'en fait sentir. On comprend bien, en effet, que l'on ne pourrait prescrire de revenir à des époques fixes, par forme de coupes secondaires ou de coupes définitives, sur les coupes d'ensemencement précédemment faites, sans s'exposer à entraver 
la régénération ou le développement des jeunes plants; car on ne saurait prévoir à quelle époque et sur quelle étendue le repeuplement s'effectuera dans une coupe donnée. Tout ce qu'on peut faire utilement, c'est d'ordonner aux agenls d'exécution de proposer le repeuplement artificiel de tout ou parlie d'une parcelle si, un certain nombre d'années après la coupe d'ensemencement, elle n'était pas repeuplée.

Je connais des aménagements dans lesquels la possibilité, pour l'ensemble des coupes de régénération, ayant été fixée par volume, on a cru pouvoir néanmoins régler les coupes d'ensemencement par contenance. Ce système reposait sur l'hỵpothèse que le volume de la coupe d'ensemencement, ainsi délimitée, ne s'élèverait jamais au-dessus du chiffre de la possibilité. Or cetle hypothèse gratuite ne s'est pas toujours vérifiée ct les agents d'exécution se sont trouvés dès lors dans l'alternative : ou de dépasser le chillre de la possibilité, ou de donner à la coupe d'ensemencement une étendue moins grande que celle qui avait été fixée. En outre, il s'est présenté souvent ' des cas où il était plus urgent de faire une coupe secondaire ou définitive qu'une nouvelle coupe d'ensemencement, et cependant il y avait obligation de procéder à celle-ci pour obéir aux prescriptions de l'aménagement. Il faut donc, on le voit, adopter l'une ou l'autre des deux possibilités; mais ne pas chercher à les faire marcher ensemble, car on n'y réussirait pas. 
Quant aux coupes soit de taillis, soit d'éclaircie de nettoiement ou de recépage, soit préparatoires, ì faire dans les autres affectations que celle qu'on régénère, elles sont toujours basées sur la contenance; cela ne fait pas question.

Nous avons reconnu, quand nous nous sommes occupés du règlement spécial des exploitations à faire dans la première période de la rérolution applicable aux forêts à régulariser, qu'il pouvait être utile de dresser deux tableaux : l'un pour les coupes par volume, l'autre pour les coupes par contenance. S'il s'agit des conversions, ces deux tableaux sont encore plus utiles, parce que les procédés de culture qu'impliquent les conversions, sont plus variés. Ces tableaux - ne présentent du reste que le développement des indications du tableau général qui se rapportent à la première rutation de la révolution préparatoire ou à la première période de la révolution principale quand il n'y a pas de révolution préparatoire, et on en retrouvera les modèles aux pages 322 et 323 de ce volume. 


\section{QUATRIÈME ÉTUDF.}

DE LA DIVISION D'UNE FORETT EN MASSES, E.N SECTIONS, EN SÉRIES, DES AMÉLIORATIONS, DE LA RÉDACTION DU PROJET D'AMÉNAGEMENT, DE L'APILICATION ET DU CONTROLE.

\section{CHAPITRE PREMIER.}

DES MASSES, DES SECTIOIS, DES SERIES.

ARTICLE PREMIER.

OBSERYATIONS PRÉLIYINAIRES.

La science de l'aménagement serait une science difficile à comprendre, si l'on voulait approfondir dans tous leurs détails, au fur et à mesure qu'elles se présentent, les différentes questions dont elle réclame l'examen.

Je me suis appliqué, dans ces études, à dégager les principes fondamentaux, des objets secondaires qui auraient pu en compliquer la démonstralion, et en rendre l'intelligence laborieuse. J'ai réduit ces 
principes à leur plus simple expression, dans la pensée que c'était le meilleur moyen pour faire saisir au lecteur les rapports qui les lient. Mon but était surtout de mettre en relief l'enchaînement logique des opérations que j'avais à exposer; de montrer comment elles se commandent successivement l'une l'autre; et je crois que si j'avais atteint ce but, $j$ 'aurais répondu à tout ce que l'on peut exiger d'un travail qui est plutôt un programme qu'un traité sur la matière.

Ces observations préliminaires expliquent pourquoi j'ai fait imprimer l'ćlude sur le partage de la forêt en masses, en seclions, et en séries, après les autres.

C'est que ce partage, malgré son importance dans la pratique, est, au point de vue de la théorie, un de ces objets secondaires, qu'il n'est pas indispensable de connaître pour comprendre le mécanisme de l'aménagement.

J'entends par masse une partie de forêt qui, à priori, sans qu'il soit besoin d'une reconnaissance préalable, à raison de sa grande étendue, de sa situation topographique, de ses débouchés, peut être considérée comme indépendante du surplus. Quand une vaste forêt, comme celle d'Orléans par exemple, présente plusieurs parties dans ces conditions-là, il convient tout d'abord de la diviser en autant de masses distinctes que l'on envisage comme des forêts à part; mais cette division dont les bases sont évidentes par elles-mêmes, ne réclame aucun développement et je n'en parlerai pas davantage. 
On entend par section une partie de forêt ou de masse qui se distingue du surplus par le mode d'exploitation (taillis, futaie régulière, futaie jardinée etc.), auquel elle doit être soumise. Pour savoir combien il y a lieu de former de sections dans une forêt, il faut en avoir fait une reconnaissance générale. Cependant, ici encore, les principes d'après lesquels on se règle dans le partage d'une forêt ou d'une masse en sections, sont trop simples pour qu'il soit nécessaire de nous y arrêter.

Enfin, on entend par série, une partie de forèt, de masse ou de section, destinée à être soumise à un plan spécial d'exploitation et à fournir par conséquent une suite de coupes annuelles.

La division d'une forêt en séries est une opération souvent désirable, quelquefoís nécessaire, mais qui, dans certains cas, pourrait avoir des inconvénients.

ARTICLE II.

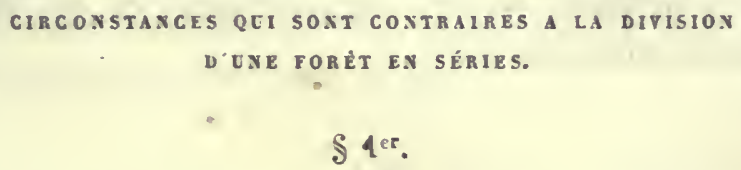

Impossibilité de former des séries compactes, indérendantes l'une de l'autre.

La division d'une forêt en séries présenterait des inconvénients, si les diverses classes d'âge de cette 
forêt étaient distribuées de façon qu'on ne pût, sans déroger aux règles, aux principes qui doivent présider à la formation des affectations, constituer des séries compactes, faciles à distinguer les unes des autres.

Supposons, par exemple, une forêt composée de la manière suivante:

La classe des bois exploitables comprend cinq parcelles $a, b, c, d, e$, dont les trois premières $a, b, c$, contiguës et situées à l'extrémité nord de la forêt, sont séparées des deux dernières $d$, $e$, également contiguës, et situées à l'extrémité sud, par des parcelles appartenant à d'autres classes d'âge.

Si l'on voulait établir deux séries dans cette forêt, il faudrait, dans le cas où les deux parcelles $d$ et $e$ ne seraient pas assez grandes pour completer la première affectation de l'une de ces séries, y ajouter une partie des parcelles $a, b, c$. Chaque série ne formerait pas une masse compacte. On serait exposé à confondre les exploitations de l'une avec celles de l'autre; la régularité des opérations et l'efficacité de la surveillance seraient compromises; et ce, sans aucune compensation.

\section{$\$ 2$}

Étendue trop petite de la forêt.

La division d'une forêt en séries présenterait des inconvénients, si l'étendue de cette forêt n'était pas 
assez grande, pour que celle de chaque série comportât une coupe annuelle qui pût se concilier avec l'intérêt de la reproduction, celui de la surveillance et l'économie des exploitations.

Ainsi, pour ce qui concerne la reproduction, il est clair qu'une coupe d'une contenance donnée, soit dans les taillis, soit dans les futaies, aurait moins à souffrir du couvert des arbres voisins, que deux ou plusieurs coupes séparées, contenant ensemble le même nombre d'hectares, puisque ce couvert s'étendrait sur une surface relalivement moins grande dans la première que dans les autres.

Quant à la surveillance, la multiplicité des exploitations en augmente naturellement les difficultés.

Enfin, au point de vue économique, si la mise en vente de coupes trop considérables a l'inconvénient de restreindre le nombre des adjudicataires qui peuvent y prétendre, il est, d'un autre côté, à remarquer que l'exploitation d'une coupe donne lieu à des frais généraux, indépendants de la quantité de bois à abattre, et qu'il importe beaucoup, tant dans l'intérêt du trésor que dans celui des adjudicataires, de ne pas multiplier ces frais sans nécessité.

\section{\$3.}

Exigences de l'assiette et de la vidange des coupes.

La division d'une forêt en séries présente des inconvénients, lorsqu'elle est inconciliable avec l'ap- 
plication des règles sur l'assiette et la vidange des coupes.

Si on ne pouvait, par exemple, d'après la position respective des classes d'âge, composer deux séries qu'en les superposant sur une rampe escarpée, qui ne permettrait pas d'établir des moyens spéciaux de vidange pour la série supérieure, on manquerait évidemment de l'une des conditions nécessaires pour une utile distribution en séries.

\section{A R T I G L E III.}

CIRCONSTANCES QUI RENDENT AVANTAGEUSE OU NÉCESSAIRE

LA DIVISION D'UNE FORÊT EN SÉRIES.

\section{$\S 1^{\mathrm{er}}$.}

Diversité des modes d'exploitation.

Le mode d'exploitation adopté pour certaines parcelles de la forêt à aménager, peut ne pas être applicable à d'autres; or, de même qu'il n'est pas absolument défendu de comprendre dans une seule série d'exploitation, des parcelles exploitables à des âges différents ; de même il est permis, à la rigueur, d'y comprendre des parcelles dont les modes de traitement ne seraient pas semblables, lorsque ces modes sont d'ailleurs réguliers; mais il est évident qu'on ne doit s'y résoudre qu'en cas de nécessité bien démontrée, car la diversité des modes d'exploi- 
tation est difficilement conciliable avec les règles sur l'assiette des coupes et l'établissement du rapport soutenu.

Lorsque des différences dans les modes de traitement des parcelles qui composent une forêt sont inévitables, il est donc désirable qu'on puisse obvier aux inconvénients de tous genres qu'elles seraient susceptibles d'entraîner, au moyen de la constitution d'autant de séries qu'il y a de modes particuliers.

L.a division d'une forêt en séries devient absolument nécessaire quand cette forêt renferme des bois exploitables, les uns en futaie régulière, les autres en futaie jardinée. On ne conçoit, en effet, aucun moyen de concilier les exigences de l'aménagement avec celles qui sont inhérentes à la dernière de ces méthodes.

\section{\$2.}

Dirersitê des åges d'exploitabilité.

Des considérations analogues à celles que je viens d'exposer, à propos du mode d'exploitation, sont applicables à une forêt composée de parcelles dont l'exploitabilité ne sẹrait pas la même.

\section{\$3.}

Morcellement des classes d'age.

Lorsqu'une forêt est très-vaste d'une part, et que 
de l'autre, les parcelles comprises dans une même classe d'âge n'y sont pas toutes attenantes les unes aux autres, on pourrait se trouver dans l'obligation de scinder une ou plusieurs affectations, afin d'éviter de couper des bois à un âge trop éloigné de celui de . leur exploitabilité, si l'on n'avait pas recours à la division de la forêt en séries.

Dans la forêt $\mathrm{F}$, la classe des bois de 1 à 20 ans comprend des massifs situés, les uns à l'extrémité nord-est de la forêt, les autres à l'extrémité sud-ouest. Si l'on ne voulait établir qu'une série d'exploitation dans cette forêt, la dernière affectation se composerait de deux parties fort éloignées l'une de l'autre. Or, quoiqu'un morcellement de ce genre soit quelquefois admissible, il ne l'est cependant jamais sans inconvénients, et il est bon de l'éviter autant que possible. Au cas particulier, le partage de la forêt en deux séries distinctes l'èverait l'embarras.

Les séries donnent donc les moyens de masser les affectations et rendent, par conséquent, plus facile l'application des règles sur l'assiette des coupes.

$\$ 4$.

Exigences du rapport soutenu.

Une autre difficulté, et des plus graves, que les séries permettent de surmonter, dans la formation du plan d'exploitation, c'est celle qui résulte, pour 
l'établissement du rapport soutenu, des différences qu'on rencontre dans les conditions de la végétation.

Nous savons que pour assurer le rapport solltenu lorsque ces différences tendent à le compromettre, on a imaginé de rendre les contenances des affectations inversement proportionnelles à leur puissance productive; mais nous savons aussi que cette opération est fort embarrassante et fort incertaine, à cause de l'insuffisance des données nécessaires pour la fixation des coefficients de production.

Il est donc très-désirable que l'on puisse se dispenser de recourir à ces coefficients et à ces contenances proportionnelles. Or les séries sont de nature à leur enlever tout objet et par' conséquent toute utilité. Que l'on admette la possibilité de former autant de séries qu'il y aurail de différences dans les conditions de végétation relatives au sol, à l'exposition, aux essences, etc., etc., et le produit de chaque affectation devenant directement proportionnel à la contenance, le rapport soutenu sera assuré par l'égalité des contenances.

C'est surtout à ce point de vue que la division d'une forêt en sẻries est recommandable.

En effet, où sont les chances d'erreur dans la fixation des coefficients de production?

Elles ne sont pas dans la constatation de ce fait qu'il y a des différences dans les conditions de la végétation; elles sont dans l'appréciation, dans la détermination des effets que ces différences sont susceptibles de produire; or, pour le partage de la forêt en 
séries, cette appréciation, cette détermination exacte est sans utilité ; on forme par exemple une série avec les peuplements exposés au sụd, une autre série avec les peuplements exposés au nord, parce qu'il est incontestable que l'exposition exerce une grande influence sur la végétation et en conséquence sur la production, et cela suffit. Il n'est pas nécessaire d'ailleurs de préciser cette influence, d'en calculer le résultat matériel.

\section{$\$ 5$.}

Application, contrôle et rectification de l'aménagement.

Les erreurs d'assiette sont plus à craindre et plus graves dans une grande affectation que dans une petite. Les outre-passes de possibilité sont plus faciles à constater pour la seconde que pour la première. On pourrait marcher plusieurs années dans une affectation de 1000 hectares par exemple, sans soupçonner qu'on y prend plus de bois que n'en comporte la possibilité réelle, tandis que si l'affectation n'a qu'une soixantaine d'hectares et que les coupes y soient exagérées, un œil tant soit peu exercé ne tardera pas à le reconnaître.

La rectification de l'aménagement est enfin plus prompte dans une forêt, lorsqu'elle contient plusieurs séries que lorsqu'elle n'en contient qu'une; car il se peut, dans le premier cas, que cette rectification n'affecte qu'une série, et n'entraîne par conséquent 
le remaniement de l'aménagement que pour une portion de la forêt; tandis que, dans le second, c'est pour la forêt tout entière que l'aménagement est à remanier. Il en est des séries comme des arches d'un pont: un vice de construction, lorsqu'il y a plusieurs arches, peut n'occasionner la reconstruction que d'une partie du pont, tandis que lorsqu'il n'y a qu'une arche, on est souvent forcé de refaire le pont en entier.

On voit par ces exemples que la division d'une forêt en séries est propre à assurer l'application, le contrôle de l'aménagement, et à en simplifier la rectification.

Nous n'avons envisagé jusqu'ici que les circonstances qui concernent ou les conditions de la végétation, ou l'ordre intérieur de la forêt; mais il peut s'en présenter d'autres parmi lesquelles il y en a quelques-unes qui méritent d'être signalées.

$$
\S 6 .
$$

Diminution des frais de transport des bois.

Le transport des produits pourrait être singulièrement contrarié par la constitution de la forêt en une seule série. Ainsi supposons que l'on ait à aménager une forêt comme ce!le de Fontainebleau, dont la contenance est de 16 à 17,000 hectares, ce qui implique que, pour la traverser, il faut parcourir, en 
moyenne, 12,000 mètres, il ne sera certainement pas indifférent- d'avoir une seule série d'exploitation, ou d'en avoir plusieurs; car, dans un cas, les produits principaux ne seront offerts à la consommation que sur un seul point, tandis que dans l'autre, ils le seront sur plusieurs.

J'admets que ces produits se partagent par portions égales entre les populations environnantes, la distance moyenne à parcourir pour qu'ils arrivent au consommateur devra se compter, si l'on n'a qu'une série, à partir du point central de la forêt, et s'il y en a plusieurs, à partir du point central de chaque série, c'est-à-dire d'un point plus rapproché du consommateur, de toute la distance existant entre le centre de la forêt et le centre de chaque série.

La division d'une forêt en plusieurs séries permet donc de réaliser une économie sur les frais de transport des produits.

\section{$\S 7$.}

Égalisation du prix de revient des bois aux lieux de consommation.

La division d'une forêt en séries a en outre pour effet, avantage non moins appréciable que le précédent, de rendre, en multipliant les centres de production et en les rapprochant des lieux de consommation, le prix du bois moins variable. 


\section{$\S 8$.}

Besoins de la consommation en bois de direrses especes.

La formation des séries permet encore d'éviter des intermittences dans la production des bois de diverses espèces.

Lorsque l'industrie réclame des bois d'une nature particulière, il vaut infiniment mieux les lui donner chaque année, par petites portions, que périodiquement, à de longs intervalles, par grandes quantités. Qu'elle ait besoin par exemple de bois propres à la fabrication des sabots, si l'on n'avait pas soin de former une série d'exploitation avec les essences propres à cet usage, il pourrait s'écouler plusieurs années sans qu'on eût occasion d'en exploiter, et le volume considérable qu'on en couperait, de temps en temps, ne réparerait pas le préjudice causé par les années de privation, tant aux ouvriers qu'aux consommateurs.

\section{s9.}

Droits d'usage.

La division d'une forêt en séries devient enfin une nécessité, lorsque des droits d'usage portent sur une portion déterminée de cette forêt ou réclament des produits spéciaux, et cette nécessité est assez 
évidente par elle-même pour que je puisse me dispenser de la justifier.

\section{ARTIGLE IV.}

\section{TABLEAU DES SÉRIES .}

La série intervient en définitive comme agent simplificateur dans les travaux d'aménagement, et le partage d'une forêt en séries est une opération fondamentale dont dépend en grande partie le succès de ces travaux; aussi ne saurait-on y procéder avec trop de maturité.

Lorsque les séries sont arrêtées, on en fixe les limites sur le terrain et sur le plan, et on en dresse le tableau conformément au modèle ci-joint. 
DES SÉRIES.

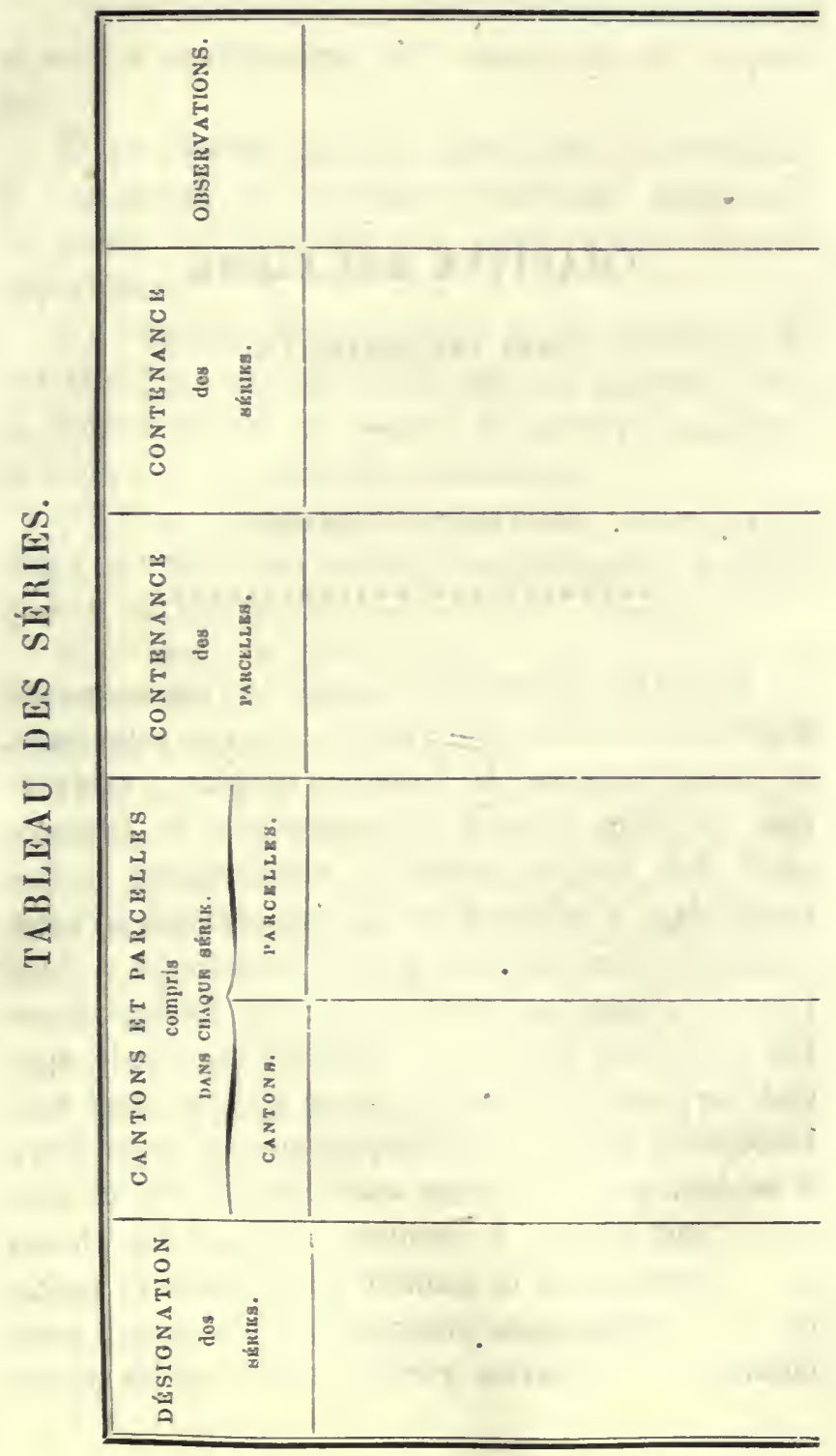




\title{
CHAPITRE DEUXIÈME.
}

\author{
DES AMÉLIORATIONS.
}

ARTICLE PREMIER.

OBSERVATIONS PRÉLIMINAIRES.

Toutes les réformes que comporte l'aménagement. d'une forêt ont pour but l'amélioration de cette forêt, et, en conséquence, la formation du plan d'exploitation lui-même devrait rigoureusement et logiquement être rangée parmi les améliorations et être traitée dans la même étude. L'aménagement ne comprendrait alors que deux parties principales : l'une. pour la statistique, l'autre pour les améliorations. J'ai cru devoir cependant consacrer une étude spéciale au plan d'exploitation, parce que cet objet était susceptible de longs développements et qu'en outre il se distingue des autres améliorations, en ce sens qu'il tend surtout à modifier, non pas les choses elles-mêmes, mais la manière de les utiliser; tandis que les améliorations proprement dites portent principalement, et souvent exclusivement, sur les choses 
et sur les modifications qu'il serait utile d'y apporter.

En parcourant successivement tous les articles de la statistique, on reconnaitra facilement quels sont les points sur lesquels des améliorations seraient opportunes.

S'il résultait par exemple du cahier descriptif de la statistique, que les limites sont en mauvais état, on proposerait de les réparer, et on ferait connaitre la dépense que ce travail nécessiterait.

S'il était constaté que lo gibier fait beaucoup de tort à la forêt ou aux cultures environnantes, on indiquerait les mesures à prendre pour y remédier.

Si le débit des bois exigeait l'etablissement de nouvelles scieries, la réparation des anciennes, on dresserait le devis de ces travaux.

Je ne saurais prévoir ici toutes les imperfections, toutes les lacunes qui pourraient exister dans une forêt, et qui devraient faire l'objet de propositions spéciales tendant à les réparer. Je me bornerai à donner quelques courtes explications sur les travaux dont l'utilité est la plus grande et l'opportunité la plus fréquente. Ces travaux sont relatifs au sol, au peuplement, aux moyens de vidange, à la conservation et. à l'entretien de la forêt. 
ARTICLE II.

AMÉLIORATIONS RELATIVES AU SOL.

Dans l'agriculture proprement dite, on améliore le sol par divers moyens et principalement par les amendements et les engrais artificiels. En sylviculture, les amendements et les engrais doivent être le résultat de la culture elle-même; c'est l'humus qui les constitue. Les expériences, très-peu nombreuses d'ailleurs, qui ont été exécutées pour démontrer les avantages de l'introduction dans les forêts de substances minérales particulières, ne paraissent concluantes ni au point de vue cultural ni au point de vue économique. On a essayé aussi de constater le bon effet des irrigations sur les bois, et on a obtenu de magnifiques résultats comme rapidité d'accroissement; mais on ne connait pas l'influence de ces irrigations sur la qualité des arbres, et on n'a pas non plus établi la balance entre le profit et les frais de ce moyen artificiel d'amélioration qui, dans tous les cas, ne saurait être praticable que très-exceptionnellement ${ }^{1}$.

Je pense donc qu'en ce qui concerne le sol, l'amélioration la plus recommandable est en définitive

4. Il faudrait les multiplier. M. Chevandier a publié à ce sujel, sur l'effet des irrigations et d'autres points impor'ants d'économie forestière, des mémoires intéressants, que mes lecteurs connaissent sans doute. 
l'assainissement. Touit le monde sait que dans les terrains trop humides, les arbres sont d'une qualité \rès-inférieure; que les graines pourrissent et qu'en conséquence le repeuplement naturel ne se fait pas.

Dans les propositions qu'ils auront à présenter à ce sujet, les agents aménagistes indiqueront les canlons et les parcelles oŭ des fossés d'assainissement. seraient nécessaires, les dimensions et la direction qu'il conviendrait de donner à ces fossés, et enfin les dépenses approximatives qu'enlraînerait leur exéculion.

\section{ARTILLE III.}

A ÉLIORITHOUS BELATINES AT PETPLEYEXT.

Les agents clıargés de l'aménagement sortent de leurs altributions lorsqu'ils s'avisent de donner des instructions, sur la manière de procéder-aux semis el aux plantations. Leưr tàche se borne à indiquer les parcelles et les époques ou les repeuplements artificiels doivent êtıe effectués, et les frais auxquels ils donneront lieu.

Il semblerait naturel, au premier abord, de prescrire le repeuplement inmédiat de tout vide existant dans la forèt. 'Toutefois, une semblable prescription pourrait entraîner de graves inconvénients, d'abord à cause des dépenses qu’elle occasionnerait, et ensuite à cause des conditions défavorables dans les- 
quelles serait fait le repeuplement des vides compris dans les affectations intermédiaires.

C'est donc seulement dans les affectations des périodes extrêmes, qu'il est toujours opportun d'effectuer immédiatement le relsoisement des vides. Pour la première affectation, cetle opportunité est évidente. Pour la dernière, elle n'est pas moins sensible, puisque çette affectation ne contient que de jeunes peuplements avec lesquels cenx qu'on aura créés ne tarderont pas ì s'harmoniser.

Quant aux affectations des périodes intermédiaires, le reboisement des vides qu'elles renferment est loin d'être aussi urgent, et souvent même il peut être impraticable immédiatement à cause du couvert des arbres voisins. Cependant, s'il y exislait de grands espaces dénudés, il conviendrait de chercher à les utiliser en y implantant des essences susceptịbles d'être exploitées, à l'époque où l'affectation dont elles feraient partie arriverait en tour d'exploitation.

Les aménagistes pèseront ces considérations et feront connaître en conséquence, par rang d'urgence, la siluation, l'étendue, et les frais probables des repeuplements artificiels à faire dans le cours de la première période.

IIais s'il est nécessaire de créer des peuplements artificiels dans une forêt, il est également nécessaire d'y créer des pépinières, à moins que les années de semence n'y soient fréquentes et les semis faciles, ce qui est exceptionnel pour les bois durs. 
Les aménagistes auront donc à désigner les endroils où ces pépinières devront ètre établies, la contenance qu'on leur donnera et les frais qu'elles occasionneront.

\section{ARTICLE IV.}

AMÉLIORATIOXS FELATIVES ACX VOIES DE VIDANGE.

La tendance de l'homme sur cette terre est d'agrandir incessamment son empire sur la matière. Un économiste a dit avec raison que l'industrie, c'était le développement de l'homme, l'émission de sa pensée. En effet, un produit industriel quelconque n'est pas autre chose que l'expression-matérielle, la matérialisation, qu'on nous pardonne ce néologisme, de cette pensée.

Mais pour approprier ainsi les choses à ses -besoins, il faut ou aller à elles, ou les faire venir à soi. Le premier cas se présente dansl'enfance des sociétés. L'homme va planter sa tente sur les points qui lui offrent le plus de ressources. Ces ressources épuisées, il va se fixer sur un autre point. Tant qu'il est condamné à celte vie errante, il progresse peu. Les Arabes nomades en fournissent la preuve; mais dès qu'au moyen des voies de transport, il est parvenu à déplacer les choses, son développement marche rapidement, et le beau idéal serait réalisé si, par des procédés quelconques, il pouvait grouper autour de son habitation tous les matériaux propres à satisfaire 
ses besoins, ses goûts, ses désirs. Malheureusement, il est bien éloigné de ce beau idéal, surtoul pour ce qui concerne les bois. Non-seulement nous avons encore en France des forêts que l'on ne peut pas exploiter, mais dans celles que l'on exploite, l'absence ou l'imperfection des roies de vidange oblige de carboniser ou de façonner sur place une grande partie des produits, afin de réduire autant que possible leur volume et leur poids ${ }^{1}$. Or, la carbonisation est une manière encore barbare d'utiliser le combustible ligneux, et le façonnage en forêt fait courir au fabricant le risque de ne pas donner au bois la forme qui conviendrait le mieux au consommateur.

Sur les chemins vicinaux ou d'exploitation, les frais de traction par voiture peurent être évaiués, en moyenne, à $7 \mathrm{fr} .50 \mathrm{c}$. par mètre cube de bois. pour une distance de 20 kilomètres. Il en résulte que si le mètre cube est estimé dans la coupe $40 \mathrm{fr}$. pour le bois de service et $10 \mathrm{fr}$. pour le bois de cliauffage (à raison de 2 stères de 5 fr. chacun par mètre cube), le montant des frais dont il s'agit est à la valeur du mètre cube de bois de service dans la proportion de 18 pour 100 , et à celle du mètre cube de bois de cháuffage dans la proportion de 75 pour 100 , de

1. La plus grande partic des bois de construction que nous recevons de l'étranger, et nous en recevons chaque année pour près de 200 millions de francs, se consomment dans le Doubs, le Jura, le Haut et le Bas-Rhin, e'est-à-dire'dans les départements les plus boisés. (Note de la $2^{\text {e }}$ édition.) 
sorte que quand le lieu de consommation est à 30 kilomètres de la forêt, il n'y a plus aucun profit à y transporter le bois de chauffage.

On estime que les frais de transport entrent en moyenne pour moitié dans le prix du bois rendu au centre de consommation, et comme on a calculé, d'autre part, que ce prix, s'élevait par année, à la somme de 500 millions, il en résulte que le transport des bois, dans notre pays, occasionne une dépense annuelle de 250 millions. On voit par là combien la société est intéressée à l'amélióration des moyens d'exportation des produits forestiers. Quand on a élevé une statue à Jean Rouvet, l'inventeur du flottage sur la Cure (Yonne, 1549), on a reconnu et récompensé un des plus grands services qu'un citoyen pût rendre à son pays.

En définitive, les voieś de transport font évidemment défaut aux bois, et pourtant ils en ont un plus grand besoin que les autres produits; d'abord, parce qu'ils sont plus éloignés des populations; ensuite, parce que les forêts ne sont pas susceptibles de se grouper cormme les cultures arables autour des habitations. Et s'il est viai qu'à raison du morcellement des héritages, une grande partie des productions de la terre est consommée par les cultivateurs euxmêmes, il n'en est pas de même pour les bois, dont la moindre part au contraire reste au propriétaire ou au régisseur.

De toutes les voies qui peuvent servir au transport des bois, celle qu'il conviendrait surtout d'amé- 
lioler, c'est le cours d'eau. Un cheval traîne péniblement 1,000 kilogrammes sur une bonne route. D'après M. Michel Chevalier, un homme en tire aisément 150,000 sur un canal. Cet exemple suffit pour prouver l'immense utilité qu'aurait l'appropriation de nos cours d'eau au transport des bois et pour justifier les regrets que doit inspirer notre négligence à cet égard.

La France ne possède que $\mathbf{1 1 , 0 0 0 ~ k i l o m e ̀ t r e s ~ d e ~}$ cours d'eau navigables, et sur les canaux, le transport des bois est entravé par l'étroitesse des biefs, d'une part, et l'énormité des tarifs, de l'autre. Quant aux cours d'eau flottables, qui pour la vidange sont d'une si grande ressource, c'est à peine si on en compte 3,000 kilomètres. Cette situation est assurément bien misérable, et, avouons-le, inexcusable, dans un pays qui, sous le rapport hydrographique, a été si admirablement doté.

Le perfectionnement des moyens de vidange est donc une des améliorations auxquelles les agents chargés de faire l'aménagement d'une forêt, doivent accorder le plus d'attention.

Après avoir exposé l'insuffisance de ces moyens, ils indiqueront toutes les mesures qu'il y aurait à prendre pour y remédier.

Ces mesurez consisteront pour les routes et chemins : $\mathbf{1}^{\circ}$ dans la rectification ou la réparation des voies existantes; $2^{\circ}$ dans la création de voies nouvelles, s'il y a lieu.

Dans l'un comme dans l'autre cas, il faudra ré- 
diger un avant-projet des travaux à faire; fixer la direction et les principaux points de passage des voies nouvelles; indiquer la largeur et la pente qu'il conviendra de leur donner; justifier leur ulilité et pour cela montrer qu'clles seront susceplibles de satisfaire à toutes les exigences de la vidange; établir leur degré d'urgence, d'après la marche des exploitations; dresser en.in un devis approximatif de la dépense que les travaux nécessiteront.

En ajoulant l'intérêt du capital employé à l'exéculion de ces travaux, aux frais annuels que leur entrelien réclamera, et en retranchant cette somme de celle représentant l'économie que les travaux permettront de réaliser sur le coût du transport, on aura la mesure exacle des avantages que l'Étit en retirera.

Je dis l'État, car s'il s'agissait d'un autre propriétaire, le bénéfice que les clıemins seraient susceptibles de lui rapporter, ne se calculerait pas de la même façon. Ce qu'un particulier poursuit en effet exclusivement quand il ouvre un chemin pour la v:dange de ses coupes, c'est l'augmentation du prix de ses bois sur place. Il spécule donc sur cette considération qu'à raison de la diminution des frais d'extraction, les adjudicataires lui donneront un prix plus élevé de sa chose; mais la diminution des fra:s de transport, qui est le résultat nócessaire de la création d'un chemin, ne tourne pas toujours seulement au bénéfice du propriétaire; il y en a une part plus ou moins grande dont profite le consommateur, 
quand elle a pour effet de rendre le bois moins cher; or, cette part, qui ne constitue pas un avantage pour le particulier, en constitue au contraire un pour l'État; car son intérêt ne se séparé pas de celui du consommateur.

"Si l'on a le choix entre plusieurs voies et qu'on veuille comparer leurs avantages respectifs, on sc rappellera que le prix de l'usage d'une voie dépend de trois éléments : $1^{\circ}$ les frais de construction de la voie; $2^{\circ}$ les frais d'entretien; $3^{\circ}$ les frais de traction ou le prix de la force motrice.

L'effort pour tirer 1000 kilos est de 18 kilos 50 sur une bonne route, tandis que sur un chemin de fer il est de 5 kilos 34 seulement; mais la construction d'une bonne route coûte à peine 20,000 fr. par kilomètre, tandis qu'un chemin de fer coûte 4 à 500,000 fr. pour la même distance.

\section{ARTICLE V.}

AMÉLIORATIONS RELATIVES A LA SERVEILLANCE ET A L'ENTRETIEN.

Surceillance. - Le service des forêts relatif à la surveillance laisse en général beaucoup à désirer, 1 moins peut-être à cause de l'insuffisance du personnel, que par suite de la mauvaise organisation des triages. Dans certaines localités ils sont trop étendus; dans d'autres ils ne le sont pásassez. Dans 
les forêts domaniales, la contenance de chaque lriage est en moyenne de 500 hectares. C'est une règle qui parait adoptée par l'administration et qu'on applique sans tenir assez de compte, peut-être, des difficultés plus ou moins grandes de la surveillance. II est cependant évident que les bois situés sur la rive d'une forèt sont plus exposés aux délits et plus difficiles à garder, que ceux situés au centre et dont on ne peut par conséquent sortir: qu'en traversant les autres.

L'élude de l'organisation des triages rentre en conséquence dans les attributions des aménagisles. Ils proposeront aussi, s'il y a lieu, une augmentation du' persomnel; ils désigneront l'emplacement le plus convenable pour les maisons forestières qu'il pourrait être nécessaire de construire, et ils feront connaître le chillire approximatif de la dépense.

Entretien. - Il ne suffit pas de construire des routes, il faut les entretenir, sous peine de perdre tous les fruits d'une dépense considérable. Il résulte des expériences faites par M. Morin, directeur du Conservatoire des arts et métiers, que si on représente par 1 la résistance présentée à une charrette par une bonne route empierrée, cette résistance devient égale à 4,35 pour une route empierrée, mais très-dégradée. M. Séguret a prouvé d'un autre côlé dans les Annales forestières, que sur un bon chemin, le bois de chaulfage peut parcourir en moyenne 32 lieues; sans que sa valeur soit absorbée par les 
frais de transport, tandis que sur un mauvais cliemin, il ne peut pas en parcourir plus de 5 .

Le meilleur moyen, le plus efficace et le moins coûteux, de tenir les chemins en bon élal, c'est de réparer immédialement les moindres dégradations qui s'y produisent. Pour cela, il faut des cantonniers. On en indiquera le nombre et on fera connaître aussi les frais qu'ils occasionneront.

Les routes et chemins ne sont pas les seules choses qui demandent à ètre entretenues avec soin. Les fossés, les pépinières et les plantations réclament de leur côlé des travaux, pour ainsi dire incessants, et il serait certainement fort utile d'avoir pour y procéder, des employés spéciaux. Dans tous les cas, ces travaux sont susceptibles d'entraîner chaque année des frais dont les aménagistes apprécieront le montanl.

ARTICLE VI.

ÉTIT COLLECTIF DES AMÉLIORITIONS.

Après avoir fait de chaque amélioration l'objet d'une étude particulière, il convient de former, de toutes les améliorations, un élat colleclif qui permettent de les embrasser dans leur ensemble et de voir d'un coup d'œil le chiffre total des dépenses qu'elles occasionneront, soit dans la première période, soit dans les autres; outre cet élat collectif, on-en dres- 
sera un autre tout spécial pour les repeuplements artificiels à faire dans cháque parcelle, car de tous les travaux ce sont sans contredit les plus imporlants. 


\section{CHAPITRE TROISIENE. \\ RÉdACTION DU PROJET D'AMÉNAGENENT, APPLICATION ET CONTROLLE.}

ARTIGLE PREMIER.

RÉDACTION DU PROJET D'AMÉNAGENENT.

Je crois qu'à l'aide des notions générales que j'ii exposées, les agents qui auront été chargés de l'aménagement d'une forêt, ne seront pas sérieusement embarrassés par la manière de s'y prendre pour accomplir leur mission. Toutefois, j'ai pensé qu'il pouvait être utile de terminer cette étude par l'indication des divers documents que comporte un projet - d'aménagement.

Ces documents sont de trois sortes :

Le procès-verbal d'aménagement;

Les plans et devis;

Les pièces justificatives.

Procès-cerbal d'aménagement. - Il se divise en sept parties :

Dans la première, on s'occupe de la statistique pour l'ensemble de la forêt ou de la masse; 
Dans la deuxième, de la critique du mode actuel d'exploitation, du choix du mode d'exploitation à adopter et de la division de la forêt en sections et en séries;

Dans la troisième, du parcellaire et de la description spéciale, pour clıaque série;

Dans la quatrième, de l'àge d'exploilabilité, du chơix de la révolution, de la formation des affectations normales, du règlement général et du règlement spécial des exploitations, et des améliorations spéciales, pour chaque série;

Dans la cinquième, des améliorations, pour l'ensemble de la forêl;

Dans la sixièmé, de l'assietle de l'aménagement sur le terrain (ouverture des lignes séparatives des sections, séries, affectations, parcelles, etr.);

Dans la septième, de l'état futur de la forêt et de l'examen comparé des produits tant principaux qu'accessoires, en matière et en argent, que l'on retire actuellement de la forèt et qu'on en retirera après son améuagement. On n'omettra pas de tenir compte dans cet examen, de la plus-value probable qui résultera pour les produits, de l'amélioration des voies de vidange ${ }^{1}$.

1. Je donne l'ordre des travaux d'aprẻs les errenents suivis jusqu’à présent, mais ces errements laissent à désirer parce qu'ils ne séparent pas dans l'aménagement ce qui appartient à son applicalion, de ce qui concerne sa fréparation. Quan!l le ı̇̀glement général des exploitations a été dressé, le projel d'aménagemen est lerminé en ce qui concerne la marche el la quotité des coupns el le décret daménagement pourrail ètre pro:oqu '. Une fois ce 
Il est bien certain, d'ail'eurs, que le résultat de cette comparaison ne saurait donner qu'une idée incomplète de la supériorité du régime qu'il s'agira d'adopter, puisque, comme on le sait, tous les produits du sol forestier ne sont pas susceptibles d'ètre évalués en argent. Il est probable aussi que le revenu, faible au début de l'applicalion de l'aménagement, s'accroîtra par la suite. Ce sont là des considérations sur lesquelles les rédacteurs du projet d'aménagement auront à appeler l'attention.

\section{Plans et devis. - II faut :}

Un plan général de la forêt indiquant les princi-

décret rendu, il s'agirait d'appliquer l'aménagement. Ce serait alors seulement que l'on devrait arrêter le règlement spécial d'exploitution pour la première période, règlement qui, devant reposer sur le volume pour les coupes principales, exigerait le calcul du matériel que ces coupes auraient à enlever chaque année. Aujourd'hui ce matériel est fiıé par le décrel d'aménagemcnt. C'est à tort, selon moi; il serait plus rationnel que le décret se bornàt à indiquer le rapport constant à maintenir entre le volume total exploilable dans la durée de la période et celui de la coupe annuclle. En effet, ce rapport est la seule clıse à reconnaitre et à observer pour que la possibilité ne soit pas outrepassée, et c'est à l'administration qu'il incombe de prendre les mesures nécessaires pour que l'on ne s'en écarle pas. Mais quand au lieu d'exprimer par un cliffre ubstrait, la proportion qui duit exister entre le volume de la corpe annuelle et le volume total exploitible dans le cours de la période, on exprime le volume de la coupe annuelle par un chiffre concret, par un certain nombre de mètres cubes, on s'expose à ce que la possibilité soit outrepassée, parce qu'en supposant qu'au momeut où il est déterminé, ce nombre de mètres cubes soit dans le rapport voulu avec le matériel total exploitable, utio foule d'aciidents imprérus peusent ensuite le rendre trop fort. (Note de la $2^{e}$ édilion.) 
paux mouvements du terrain, les cours d'eau, roules et chemins, les limites des cantons, elc. ${ }^{1}$;

Un-plan général et, s'il y a lieu, des plans de détail des parcelles;

Un plan fig̣uratif des limites des séries;

Un plan indiquant, pour chaque série, les limites des affectations;

Un plan indiquant le tracé des routes et chemins projetés, l'emplacement des maisons forestières, scicries, etc., à construire;

Les devis sommaires à l'appui de ces divers Iravaux.

Il est bien entendu que, lorsqu'il ne doit pas en résulter de confusion, deux ou plusieurs des plans que je viens de désigner peuvent être réunis en un seul.

Piices justificatives. - Elles se composent :

Des calculs relatifs au lever périmétral de la forêt; d'un tableau des coordonnées des points de la triangulation et d'un tableau des hauleurs de chaque station de nivellement par rapport à un point unique.

Des expériences exigées par la recherche des renseignements sur la croissance des bois, leur débit,

1. S'il n'est pas nécessâire d'aroir un plan topographique parfaitement exact pour préparer le règlement d'exploitation d'une forèt, il est cependaut désirable à d'autres points de vue, et notamment pour les travaux de la carte de France et pour ceux du cadastre, que ce plan ne laisse rien à désirer. Cne grande administration ne saurait en pareille matière viser à l'économie. (Note de la ze édition.) 
les facteurs nécessaires pour apprécier leur rendement, tant en volume brut qu'en marchandises;

Des expériences faites pour déterminer l'àge d'exploilabilité;

Des dénombrements et cubages effectués pour établir la possibilité et des tarifs adoplés à cette occasion.

\section{ARTICLE II.}

APPLICATION ET CONTROLLE DE L'AM ŚNAGEMENT.

C'est un faif malheureusement avéré quse la plupart des aménagements exécutés à grand frais, depuis une trentaine d'années, par des commissions spéciales, n'ont pas procuré tous les avantages qu'il était permis d'en allendre. Mal compris par certains agents qui n'ont pas voulu se donner la peine de les étudier, systématiquement repoussés par d'autres, ces aménagements n'ont pas loujour's été bien appliqués.

\section{Ainsi :}

On ne s'est pas conformé assez exactement, pour' l'assiette des coupes, aux prescriptions des plans d'exploitation. Comme j'ai eu déjà occasion de le faire remarquer, les agents locaux se laissant influencer par l'état du peuplement considéré en lui-même, et non dans ses rapports avec les circonstances extérieures, ont quelquefois porté les coupes principales dans des parcelles qui auraient dù seule nent être 
éclaircies et en ont éclairci d'autres qui auraient dû être régénérées.

On ne s'est pas renfermé partout dans les limites de la possibilité; soit parce qu'on a omis de tenircompte, dans les exploitations annuelles, des arbres abattus par le vent, la neige, le givre, et vendus sous forme de menus marchés; soit parce qu'on a employé, pour l'estimation des coupes; d'autres tarifs et d'autres unités de mesure que ceux adoptés par les commissions; soit enfin parce que l'on n'a pas pris le soin d'établir chaque année une balance des exploitations, qui permît de fixer la quotité des coupes de l'année suivante, conformément aux résultats de cette balance.

On a négligé, soit par incurie, soit par manque de fonds, d'exécuter les travaux d'amélioration, reboisements, assainissements, routes, etc., etc., prévus par l'aménagement; après avoir fait une coupe d'ensemencement, on ne s'est pas toujours assez préoccupé de ce qqu'elle devenait.

On n'a pas enfin provoqué les révisions quand cependant la nécessité en était démontrée.

Pour prévenir le retour d'aussi graves irrégularités, l'administration a souvent prescrit à ses agents d'indiquer sur les états d'assiette, les parcelles dans lesquelles ils se proposent de porter les coupes principales et de justifier la quotité de ces coupes, d'après le chiffre de la possibilité réglementaire et celui des coupes de toute nature de l'année précédente.

Elle a d'un autre côté invité ces agents à se servir, 
pour l'estimation des coupes, des tarifs et des unités de mesure, adoptés par les commissions; sauf à faire une autre eśtimation spéciale pour la vente;

Mais tout cela ne suffit pas; il importe maintenant qu'elle les oblige à tenir pour chaque forêt àménagée un sommier de contrôle; il est même à désirer qu'il en soit tenu un également à la Direction générale.

Le modèle de ce sommier a été récemment arrêté par l'administration. Diviśé en trois parties distinctes: la première pour les exploitations, la deuxième pour les travaux, la troisième pour les recettes et les dépenses, il permettra de juger d'un coup d'œil, nonseulement des soins apportés dans l'application des aménagements, mais encore des résultats pécuniaires de cette application, et sous ce dernier rapport, il ne sera pas moins utile que sous le premier, car il fournira les moyens de rectifier les appréciations erronées auxquelles la gestion des forêts soumises au régime forestier a souvent donné lieu, au point de vue financier. Rien de plus commun, par exemple, que d'entendre dire que les forêts coûtent trop relament à ce qu'on en retire; et en effet, si l'on considère en bloc, sans s'occuper de leur nature, les dépenses qu'elles occasionnent, il est certain que ces dépenses dépassent de beaucoup la proportion qu'il est raisonnable de maintenir entre le revenu et les frais d'administration d'un immeuble; mais il convient de faire remarquer que, parmi les travaux dont l'aménagement d'une forêt met la nécessité en évi- 
dence, il y en a, et ce sont les plus considérables, qui sont des travaux de premier établissement, c'està-dire des travaux qui, ayant pour objet la réparation, la reconstitution, la mise en état du domaine, ne sauraient entrer en ligne de compte dans la comparaison du revenu avec les dépenses annuelles d'entretien.

Il s'est rencontré des sociétés industrielles qui, pour enfler leurs dividendes, portaient au comple de premier établissement des sommes qui appartenaient à l'entretien et aux frais d'exploitation. C'était un tort très-grave. Pour les forêts, on est dans l'usage, au contraire, de porter au compte de l'entretien ce qui appartient au capital de premier établissement, et de cette manière, on diminue le revenu net. C'est un tort dans le sens opposé et qui n'a pas peu contribué à répandre de très-fausses notions sur l'économie avec laquelle les forêts sont gérées. Il sera facile, désormais, de rétablir la vérité sur ce point, au moyen des états que les agents auront remplis. Néanmoins, ces états ne répondront pas à tous les desiderata qui se rapportent à la justi-. fication du service forestier: Ils sont destinés seulement à la constatation des faits qui peuvent se traduire par des chiffres. C'est beaucoup, mais ce n'est point assez, et il est à désirer que l'administration s'occupe maintenant de faire recueillir toutes les observations qui, en dehors des renseignements de pure comptabilité, seraient d'une réelle utilité pour la culture forestière et pour les intérêts qui s'y rat- 
tachent. On consignerait ces observations dans des cahiers spéciaux qui formeraient autant d'annexes du sommier de contrôle. J'en indiquerai onze qui me paraîtraient particulièrement utiles et qui seraient relatifs :

Le premier, à la floraison et à la fructification des arbres. On indiquerait les essences pour lesquelles elles ont été le plus abondantes, et les conditions où se trouvaient ces essences sous le rapport du sol, du climat et de la consistance du peuplement.

Le deuxième, aux expériences sur l'accroissement, dont j'ai parlé à la page $\mathbf{1 5 0}$ de ces études. Je crois qu'il y a eu quelques travaux entrepris à ce sujet. Que sont-ils devenus? Je l'ignore. En tout cas, ces travaux exceptionnels dus à l'initiative d'un petit nombre d'agents, eussent été tout à fait insuffisants. Il conviendrait d'en exécuter d'après des règles uniformes dans les différentes régions de la France, au moins pour les essences principales. Ce serait le seul moyen de se procurer des bases certaines pour la détermination de l'âge d'exploitabilité et de la durée des révolutions applicables suivant les sols, les climats et la nature du peuplement.

Le troisième, aux exploitations de tout genre: coupes de régénération, coupes d'amélioration, élagages, extractions de souches. On ferait connaître les méthodes suivies, les procédés employés, les précautions prises et les résultats obtenus, notamment pour les coupes d'ensemencement et les élagages. 
Le quatrième, aux assainissements et aux repeuplements artificiels.

Que deviennent les nombreux fossés d'assainissement que. l'administration fait ouvrir chaque année dans les parties humides de son domaine. Quel effet ont-ils produit? Ce sont des questions auxquelles la plupart des agents, eux-mêmes, seraient embarrassés de répondre. Il en sera autrement quand, chaque année, ils seront tenus de constater dans un cahier spécial les résultats de ces travaux d'amélioration.

Quant aux repeuplements artificiels, je pourrais citer des forêts dans lesquelles ils absorbent depuis tongtemps et tous les ans de fortes sommes, quoiqu'ils n'aient jamais été suivis d'aucun succès. A quoi cela tient-il? Les agents n'en savent rien et ils n'en continuent pas moins les mêmes errements.

Que la municipalité de Paris ne se lasse pas de faire replanter à grands frais sous les épais ombrages des Tuileries, de jeunes arbres qui ne réussissent jamais et meurent infailliblement au deuxième printemps. Le mal n'est pas grand, car si les arbres meurent, il y a des gens qui vivent de leur mort et ne pourraient peut-être pas vivre sans cela. La ville de Pàris est d'ailleurs assez riche pour se payer le luxe de ces plantations perpéluellement renouvelées. Mais les repeuplements artificiels dans les forêts soumises au régime forestier ont une bien autre importance que des plantations d'agrément: La sécu- 
rité de toute une contrée peut en dépendre, lorsqu'ils ont pour but, par exemple, de consolider les terrains en montagne. Ailleurs, il est nécessaire qu'ils réussissent pour que l'application des aménagements ne soit pas compromise. Partout, ils intéressent l'avenir de nos richesses forestières déjà si appauvries. C'est dans leur jeunesse, d'ailleurs, que l'on doit soigner les arbres comme les hommes pour leur préparer une longue et utile existence, et l'administration le comprend bien puisqu'elle consacre chaque année aux repeuplements artificiels et à leur entretien, une somme considérable.

Il y a donc, en définitive, beaucoup de raisons pour que cette partie du service soit soumise désormais à un contrôle sévère et efficace. Le cahier que je propose de faire tenir aux agents le rendrait possible.

On y constaterait pour chaque repeuplement d'une certaine étendue :

L'exposition et la situation du terrain, son état à l'intérieur, à la surface, le genre de préparation qui lui aura été donnée, l'époque de celte préparation;

L'époque de la plantation ou du semis;

L'essence, le nombre, la provenance, l'âge et la qualité des plans mis en terre, les précautions prises pour assurer leur réussite;

L'espèce, la quantité et la provenance des 'graines ensemencées, les procédés employés pour l'ensemencement;

- Le coût de l'opération dans son ensemble et dans ses détails; 
Les soins et les dépenses d'entretien, binage et remplissage ;

L'étal -du repeuplement chaque année, jusqu'au. moment où il pourra être regardé comme complet.

Le cinquième, à l'estimation et à la vente des coupes :

Ce cahier, ferait connaitre les_circonstances commerciales et industrielles qui étaient de nature à réagir sur le prix du bois;

Les procédés employés pour assurer l'exactitude de l'estimation;

Les résultats comparés de la vente sur pied et de la vente des produits façonnés;

Les résultats de la décomposition du mètre cube en marchandises diverses, de sorte que l'administration pût se rendre compte-des progrès accomplis dans l'utilisation de la matière ligneuse.

Le sixième, aux routès, chemins et autres moyens de vidange.

On y consignerait tous les renseignements nécessaires pour éclairer l'administration sur leur état et la facilité qui en résulte pour la vidange;

On y examinerait les avantages et les inconvénients des différents modes (adjudication, régie ou économie) employés pour l'exécution des travaux;

On y signalerait toutes les améliorations dont le transport des produits à travers bois, le débardage en un mot, aurait été ou pourrait être l'objet.

Le huilième, au débit, au façonnage et à la carbonisation des bois en forêt : 
L'administration ignore presque complétement quelle est la plus-value que ces opérations ajoutent au bois dans la coupe même, et si on lui demandait combien un mètre cube de bois d'industrie dans telle forêt représente de journées de travail, elle ne saurait répondre. Ces journées de travail constituent cependant pour l'État un produit aussi prẹ́cieux que la somme d'argent qu'il a retirée de la vente du mètré cube en question.

Dans ce cahier, la carbonisation, l'écorçage et les scieries mériteraient de faire l'objet d'une attention particulière.

La carbonisation en forêt se fait aujourd'hui comme elle se faisait il y a mille ans. Elle est restée stationnaire depuis un temps immémorial, et cela vient peut-être de ce que les agents forestiers ont trop négligé de rechercher les améliorations dont elle pourrait être susceptible.

L'écorçage non plus n'est pas encore sorti de l'ornière de la routine. Dans ces dernières années, on a fait grand bruit d'une méthode qui consiste ì écorcer le bois vert ou sec, au moyen de la vapeur, et qui a par consécquent le grand avantage d'éviter l'écorçage sur pied dont les inconvénients sont bien cónnus. Un homme distingué, ancien inspecteur des forêts, M. de Wavrechin, a dépensé beaucoup de temps, d'argent et d'intelligence, pour propager cette nouvelle invention, sans parvenir à yintéresser l'administration des forêts. Cependant si l'écorçage à la vapeur' n'enlevait rien à la qualité de l'écorce, comme 
semblent le prouver de nombreuses expériencés, l'administration des forêts manquerait à tous ses devoirs en maintenant dans ses cahiers des charges relatifs aux coupes, la faculté pour les adjudicataires de n'abattre que lorsqu'ils sont en pleine séve, les taillis à écorcer.

Les scieries sont encore, la plupart, entre les mains de l'administration qui, chaque année, en fait même établir de nouvelles. Elle a des scieries à elle, afin de soustraire ses adjudicataires aux prétentions des particuliers propriétaires eux-mêmes de scieries, prétentions qui deviendraient exorbitantes si l'État ne leur fesait pas concurrence. C'est une raison qui a sa valeur sans doute dans la situation-actuelle des choses. Cependant il est fâcheux, en principe, que l'État se charge de travaux que les particuliers peuvent faire; d'abord, parce qu'en général il les fait moins bien et moins économiquement; ensuite, parce que son intervention dans l'industrie, n'étant pas subordonnée à la poursuite du plus grand profit net, crée aux particuliers une rivalité qui est de nature à les effrayer et à paralyser leurs efforts.

Aujourd'hui la construction d'une scierie exige encore des capitaux relativement considérables, sans compter les difficultés auxquelles donne lieu l'usage deș eaux courantes, et si l'administration des forêts n'avait pas de scieries à elle, il se formerait infailliblement pour le sciage du bois, des monopoles qui mettraient les adjudicataires des coupes à la merci de quelques spéculateurs; mais un jour viendra pro- 
bablement où la scierie locomobile à vapeur perfectionnée pourra circuler facilement à travers les forêts et s'installer sur le sommet des plus hautes montagnes; on louera alors une scierie comme on loue actuellement une charrue ou une machine à battre le blé. Quand il en sera ainsi, l'administration des forêts n'aura plus à se mêler de la question, et je crois qu'elle devrait faire quelques efforts, chercher des moyens, donner des encouragements, pour se débarrasser le plus tôt possible des soins que cette question lui impose.

Le neuvième, aux incendies, aux animaux et aux insectes nuisibles :

On y exposerait toutes les mesures prises pour prévenir ou arrêter les ravages qu'ęntraînent ces diverses causes de destruction, ravages dont l'administration ne connaît même pas toujours l'existence.

Le dixième, aux observations météorologiques (gelées, verglas, ouragans, coups de vent, orages, pluie, neige, température, pression barométrique.)

L'administration des forêts indépendamment du profit qu'elle retirerait pour elle-même de ces observations, pourrait rendre de grands services à la science de la météorologie; elle comprend un nombreux personnel qui réside dans les régions les plus accidentées et qui est par conséquent placé dans les meilleures conditions pour se livrer à des expériences intéressantes; mais il faudrait que ce personnel fût muni des instruments et des instructions nécessaires 
pour faire des observations exactes et comparables ${ }^{1}$.

Le onzième enfin, à l'influence des exploitations forestières sur le bien-être matériel et la moralité des populations environnantes.

Ce cahier ne serait pas le moins important; on y donnerait le nơmbre des bras employés par chaque nature de travail, le chiffre des salaires, l'exposé des avantages que l'agriculture retire des produits divers que la forêt lui' fournit.

1. L'école de Nancy fait depuis quelques années, sous l'babile direction du savant professeur M. Matthieu, des observations météorologiques dout l'utilité est déjà fort appréciée. 



\section{CINQUIEME ÉTUDE.}

ÉTAT NORMAL DES FORÊTS

ENVISAGÉES DANS LEUR ENSEMBLE.

TRAVAUX A FAIRE POUR LE RÉTABLIR EN FRANCE.

LOIS ET RÈGLEIENTS FORESTIERS.

ATTRIBUTIONS DE L'ADMINISTRATION FORESTIERE.

\section{CHAPITRE PREMIER.}

\section{ÉTAT NORMAL DES FORÊTS. TRAVAUX A FAIRE POUR LE RETABLIR.}

Il a été souvent question dans ce livre de l'état normal des forêts. Qu'est-ce que l'état normal des forêts? - C'est l'état de ces forêts quand elles sont, avec les autres éléments qui constituent notre planète, dans les conditions de dépendance réciproque, dans les conditions d'équilibre sur lesquelles repose l'harmonie générale de l'univers. Mais le définir ainsi, c'est dire combien nous sommes impuissants à nous en faire une notion précise : nous comprenons que de certains rapports doivent exister entre les prin- 
cipaux règnes de la nature et les composants de ces règnes; nous avons le pressentiment que rien de ce qui a été créé ne peut être impunémont détruit, que chaque objet a sa raison d'être, et que les forêts ne sont pas moins indispensables à la durée et à l'amélioration de notre globe que l'air, l'eau, le feu, etc.;' nous ne saurions d'ailleurs circonscrire exactement leur mission; notre intelligence ne va pas jusque-là. Ces forêts, ces fleuves, ces mers, ces lacs, ces innombrables quantités d'animaux, de plantes et de minéraux, qui ont été mis à notre disposition, remplissent dans la vie universelle des fonctions providentielles; nous n'en doutons pas; nous le sentons fermement, et pourtant nous sommes incapables d'expliquer les lois qui régissent ces fonctions.

Mais si nous ne pouvons remonter aux causes premières et pénétrer les desseins de la création, nous pouvons apprécier les diverses influences du milieu qui nous entoure, sur notre bien-être, et cela suffit pour que nous évitions de troubler une organisation dont Dieu seul a le secret.

L'état normal, tel qu'il nous est permis de le concevoir, est donc un état secondaire et relatif: c'est l'état qui se concilie le mieux' avec les besoins de notre vie. Toutefois, pour que cet état secondaire et relatif ne soit pas lui-même au-dessus de notre intelligence; pour. que nous soyons à même de juger sainement des altérations qu'il aurait éprouvées, il faut que nos facultés soient parvenues à un certain degré de culture. 
Au début des sociétés, obéissant à l'instinct. plutôt qu'à la raison, l'homme attaque la nature étourdiment, sans se soucier d'en respecter l'harmonie générale; seulement, comme il ne peut guère se servir que de ses mains, la nature est plus forte que lui et les blessures qu'elle en reçoit sont promptement guéries. Les conditions de la lutte sont tout autres dès que l'homme se civilise : il acquiert alors, par la science, des instruments très-énergiques pour chånger l'ordre naturel des choses, et il court le risque de le bouleverser lorsqu'il néglige de demander au développement intellectuel et moral, qui est luimème la cause de ce danger, le moyen d'y échapper. Or l'histoire prouve, hélas! qu'il s'est plus inquiété jusqu'à présent de faire acte de son pouvoir que d'en prévenir les abus, et qu'il n'a pas compris la nécessité de modérer et de régler ses entreprises sur la nature, s'il ne voulait les voir se retourner contre lui; aveuglement fatal, déplorable, dont l'origine est dans ce défaut que j'ai eu l'occasion de signaler si souvent, l'oubli de la solidarité.

Quelles sont les facultés qui grandissent chez l'homme avec la civilisation? C'est d'abord l'observation; par elle, il se rend compte de l'effet immédiat de ses actes. C'est ensuite la mémoire; par elle, il forme ce capital précieux, l'expérience, sans lequel tous ses efforts ne donneraient que des résultats éphémères. C'est enfin la prévoyance; par elle, il juge de ce qu'il doit faire ou ne pas faire dans l'intérêt de l'avenir, et c'est cette der- 
nière faculté qui lui permet d'user des autres avec la circonspection désirable. Malheureusement la prévoyance est précisément la faculté qui a toujours le plus manqué aux hommes. Et pourquoi leur a-t-elle manqué? - Par'ce qu'ils ne l'ont appliquée qu'à leurs intérêts individuels, au lieu de l'appliquer à leurs intérêts collectifs; parce qu'ils ne l'ont pas étendue, complétée par le sentiment de la solidarité. Il est incontestable cependant que ce sentiment est nécessaire pour que la prévoyance ait toute son efficacité, surtout quand il s'agit des forêts; car les suites fâcheuses d'un déboisement, qui seraient peu sensibles si on ne les appréciait que par rapport à soi et pour un temps limité, pourraientl'être beaucoup si on les appréciait par rapport à la généralité des hommes et pour un temps plus long. Les éléments n'ont pas été créés pour l'homme isolé, et il est rare qu'on en jouisse sans excès, quand on les attaque dans un but égoïste. Aussi, peut-on assurer qu'il n'y aura de garanties pour la conservation de notre globe dans un état normal, que le jour oủ la paix universelle y régnera, le jour où les mille sociélés qui l'labitent ne formeront plus qu'une seule famille; attendu que ce jour-là seulement, il sera possible d'en régler l'exploitation d'une manière adéquate aux intérêts, aux besoins réels de l'humanité envisagée dans ses générations successives. Aujourd'hui, cette exploitation rationnelle est impraticable. En supposant en effet que l'on parvînt à remplacer la prévoyance individuelle qui préside trop souvent aux actes des hommes 
dans une nation, par la prévoyance collective dans cette nation même, on n'aurait qu'une prévoyance insuffisante, à cause du manque d'entente que la rivalité des peuples a amené entre eux. S'il plaisait demain aux Suisses de déboiser les montagnes où le Rhône prend sa source, ce qui pourrait ne pas leur nuire à eux, toute notre prévoyance ne saurail aboutir qu'à régulariser le régime des affluents que ce fleuve reçoit de notre pays; elle serait donc insuffisante. $\mathrm{Si}$, pendant que nous prohibons la destruction des oiseaux, parce que ces oiseaux sont utiles à l'agriculture, on continue de s'acharner à les tuer quand ils passent sur un territoire voisin, notre prévoyance non plus ne sert pas à grand'chose. Ainsi, je le répète: tant que les hommes se feront la guerre, ils seront exposés à abuser des biens que la Providence a mis à leur portée, puisqu'ils s'interdiront de prévoir les effets de leur jouissance pour l'humanité tout entière.

Cette vérité-là n'est pas une vérité de sentiment, c'est une vérité de raisonnement. Les temps sont encore loin, malheureusement, où les hommes auront la sagesse de la pratiquer, et ce serait beaucoup déjà que dans un seul et même pays les habitants fussent amenés à comprendre qu'il y a des objets, comme les forêts par exemple, dont l'exploitation ne saurait être l'affaire de quelques individus seulement, mais celle de tous.

Où en sommes-nous, en France, à l'égard des forêts? Quelles sont les atteintes évidentes que leur 
état normal y a souffertes, et comment pourrait-on les réparer?

Il y a en France plusieurs millions d'hectares de landes et de friches dont l'agriculture proprement dite ne saurait 'tirer aucun parti, et qui, autrefois boisés, ne produisent aujourd'hui que des fièvres.

Il y a sur les bords de l'Océan d'énormes quantités de sables que les courants marins y accumulent sans cesse et qui, n'y rencontrant plus la bariière que les massifs boisés leur opposaient jadis, s'a vancent peu à peu dans l'intérieur des terres, laissant le désert derrière eux.

Il y a de vastes plaines où le cultivateur vit dans la crainte perpétuelle d'être privé d'un moment à l'autre des fruits de son travail, par les intempéries climatériques dont l'abri des forêts ne modère plus la rigueur.

Il y a des montagnes qui, ravagées par les torrents depuis qu'elles ont été déboisées, s'en vont par lambeaux dans les vallées qu'elles obstruent de leurs débris.

Il y a des fleuves dont les inondations, de bienfaisantes qu'elles étaient, lorsqu'il y arait encore des arbres pour diminuer la vitesse d'écoulement des eaux, sont devenues dévastatrices à cause de leur violence et des déjections qu'elles vomissent sur les propriétés.

Notre pays est tributaire de l'étranger pour des produits ligneux dont la privation compromettrait sa sécurité, son influence au moins. Notre marine ne 
trouve plus dans les forêts de la France la quantité de bois dont elle a besoin. On ne voit que des rapailles là où il y avait anciennement les plus belles forêts de chêne du monde, que la Providence semblait avoir tout exprès plantées auprès de nos grands ports maritimes, pour l'approvisionnement de leurs chantiers.

Enfin, sur les huit à neuf millions d'hectares de bois qui nous restent, il y en a trois qui, bien que soumis au régime forestier, sont loin de donner les produits que l'on devrait, que l'on pourrait en retirer.

Tous ces faits constituent certainement des atteintes à l'état normal de notre pays, puisque nous en souffrons et que nos descendants en souffriront encore plus que nous-mêmes, si nous n'y remédions.

Dans mon livre, je ne me suis occupé que de l'amélioration des forêts existantes, et encore l'ai-je fait d'une façon étroite, en ne considérant les forêls que dans leurs rapports avec les circonstances en quelque sorte environnantes, et quand j'ai parlé de leur état normal, j'ai voulu parler de leur ẻtat relativement à ces circonstances.

On voit donc que l'aménagement ainsi compris est un petit détail à côté des travaux qui consisteraient à restaurer le domaine forestier de la France entière; mais, par ce petit détail, on peut se rendre compte de la marche à suivre pour arrèter les bases desdits travaux.

La première chose à faire serait la statistique. 
Elle comprendrait non-seulement les forêts et les pâturages existants, mais encore tous les espaces dénudés dont l'intérêt général réclamerait le boisement ou le gazonnement.

Elle pourrait être établie par bassins géographiques et autant que possible par catégories de propriétaires.

Les renseignements y seraient classés dans l'ordre que j'ai indiqué page 22. Je n'en referai pas la nomenclature et je me bornerai à signaler ceux qu'il conviendrait de mettre en relief ou d'ajouter.

Ce seraient :

L'étal des forêts situées en montagne, et celui des forêt situées en plaine;

La distribution des essences forestières suivant les formations géologiques, suivant la latitude et suivant l'altitude;

La situation, l'étendue et l'état de conservation des terrains affectés au pâturage;

Les mêmes renseignements pour les terrains improductifs, avec l'indicalion de l'origine et des causes de leur stérilité ;

Les influences météorologiques dominantes dans chaque bassin ; leurs effets sur la santé des hommes et sur la production agricole;

Les principales voies: routes de terre, chemins de fer, canaux et rivières servant au transport du bois et le coût de ce transport par mètre cube et par kilomètre, pour chaque nature de voie;

Les cir'constances naturelles ou économiques fa- 
vorables ou contraires à la conservation des forêts ;

Les produits généraux en bois de marine, de service, d'industrie, en bois de feu, et les prix maxima et minima de ces produits sur place;

Le nombre, l'espèce et le rendement des animaux entretenus dans les pâturages. On distingucrait, dans cet article, les animaux appartenant aux populations locales, de ceux qui viennent du dehor: Dans le rendement, on indiquerait la part afférente à la laine, au lait, à la viande, le bénéfice du propriétaire du pâturage, celui du propriétaire des animaux;

Les principaux lieux de consommation des produits ligneux, et les prix de revient de ces produits;

La consómmation des services publics, de la marine militaire et de la marchande, celle des usines et des principales industries;

La nature et la valeur des produits ligneux importés et des mêmes produits exportés; l'origine des premiers, la destination des seconds;

La nature et la valeur des produits forestiers délivrés aux populations rurales;

Le nombre et le salaire moyen des ouvriers employés à l'exploitation et au débit des bois dans l'intérieur des forèts; ceux des ouvriers dans les industries en dehors des forêts, employant le bois comme matière première. laire.

Après la statistique, on procéderait au parcel-

Mais le parcellaire ne s'arrêterait pas aux petites 
différences de sol, de climat ou de végétation, qui ont une grande importance pour une forêt prise en particulier, mais qui n'en ont aucune pour toutes les forêts embrassées dans leur ensemble. Les parcelles comprendraient souvent des forêts entières. Elles seraient limitées et déterminées par les lignes de partage des eaux et des climats, par les formations géologiques, par les caractères de la végétation spontanée, par les conditions climatologiques qui caractériseraient de grands espaces. La description de ces parcelles aurait pour but, non pas de les classer dans une affectation, mais seulement de déterminer le genre de culture à leur appliquer, la nature des travaux à faire pour leur mise en valeur.

Après le parcellaire, on dresserait le plan d'exploitation. Il ne s'agirait point, on le devine, de régler la quotilé de la coupe annuelle; il s'agirait d'un objet bien plus considérable : on aurait d'abord à déterminer le périmètre des terrains dont le boisement ou le gazonnement serait nécessaire pour la conservation des sources ou comme barrière à opposer aux torrents, aux avalanches, à la divagation des cours d'eau, aux ouragans, à l'envahissement des sables, à l'invasion du territoire par des armées ennemies, et on en formerait l'état par catégorie de propriétaires.

On ferait ensuite connaitre à grands traits celles des forêts comprises dans ce périmètre, qui pourraient sans de trop grands inconvénients être traitées en taillis, celles auxquelles on devrait appliquer la 
méthode du réensemencement naturel et des éclaircies, et celles qui seraient jardinées.

On distinguerait, parmi les terrains dénudés, ceux qui devraient être boisés, de ceux qui devraient être simplement gazonnés. Sans entrer dans les détails de la culture, on indiquerait les essences à propager, soit par le semis, soit par la plantation.

Lorsque ce périmètre, dans lequel la conservation des forêts et des pâturages serait regardée comme étant de rigoureuse nécessité, aurait été tracé, on rechercherait, en dehors, les forêts qui, quoique ne présentant pas d'utilité manifeste comme sauvegarde du territoire et des intérêts généraux du pays, en présenteraient assez cependant pour qu'il fût utile d'en prévenir la destruction. Ce seraient en premier lieu toutes les forêts domaniales; car il n'eu reste certainement plus une seule dont l'aliénation ne fût pas déplorable. Ce seraient aussi, sauf un petit nombre d'exceptions, les forêts communales ; car toutes celles dont la transformation en terres agricoles était avantageuse ont été aliénées depuis longtemps. Ce seraient, ensuite parmi les bois des particuliers, ceux dont le défrichement aurait pour conséquence de rendre le sol tout à fait infertile ou de compromettre l'existence de sources qui, quoique n'étendant pas au loin leur action, sont cependant précieuses pour les populations locales.

A ces diverses forêts on ajouterait, pour compléter le second périmètre, les terrains vides, les pàturages, les paquis áppartenant à l'État ou aux communes, 
dont on ne pourrait tirer parti que par le boisement ou le gazonnement, et que l'initiative individuelle serait inhabile à mettre en valeur.

Puis viendrait l'état des travaux à faire :

$1^{\circ}$ Dans le périmètre dont le boisement ou le gazonnement serait de première nécessité;

$2^{\circ}$ Dans celui où ces travaux n'auraient qu'une utilité secondaire.

On indiquerait pour le premier périmètre, et toujours par catégorie de propriétaires, l'étendue des terrains à reboiser, celle des terrains à gazonner, le prix approximatif des terrains, et le coût des travaux, en séparant les travaux d'art, tels que les barrages, des travaux de gazonnement ou de reboisement.

On donnerait les mêmes renseignements pour le second périmètre, mais en ce qui concernerait seulement les propriétés domaniales et communales.

On ferait connaître pour ces dermières forêts les dépenses approximatives qu'entraîneraient leur délimitation, leur aménagement et leur vidange.

On exposerait d'une manière générale les réformes qu'il serait convenable d'apporter au régime économique et légal des routes nationales, départementales, vicinales, des canaux, des rivières navigables et flottables, pour faciliter le transport des produits forestiers.

On examinerait les entraves mises au commerce des bois par les droits de navigation, de douane et d'octroi, ainsi que les conditions faites à la propriété forestière par l'impôt foncier, et on signalerait les 
mesures à prendre pour supprimer les unes et améliorer les autres.

Voilà un aperçu des travaux qu'il y aurait à exécuter, afin de remettre le territoire de la France, au point de vue des forêts et des pâturages, dans un état normal. On ne saurait en concevoir qui pussent contribuer plus que ceux-là au bien et à la prospérité de notre pays. On les a, du reste, déjà commencés, et sur un point ils sont même très-avancés : dans quelques années, les dunes seront reboisées, mais, après cette opération, que d'autres resteront encore à faire! L'administration des forêts, qui est la mieux outillée pour mener à bonne fin cette grande entreprise, l'est-elle suffisamment? Je ne le crois pas. Je veux dire par là qu'elle ne trouverait ni dans les lois, ni dans le personnel dont elle dispose, ni dans les fonds qu'on lui alloue, toutes les ressources dont elle aurait besoin. Je ne saurais, sans sortir tout à fait du cadre de ces études, examiner les réformes dont l'organisation de l'administration forestière serait susceptible. Je ne dirai rien non plus du budget, chose variable qui n'a rien à faire avec la science; mais je jetterai un coup d'œil sur les lois, afin de montrer leur insuffisance et les points principaux sur lesquels il conviendrait de les modifier. 


\section{CHAPITRE DEUXIÈME.}

LOIS ET RÈGLEMENTS FORESTIERS. ATTRIBUTIONS DE L'ADMINISTRATION FORESTIÈRE.

Les lois et les règlements dont l'exécution est confiée à l'administration forestière, si l'on en excepte les lois récentes sur le reboisement et le gazonnement des montagnes qui ne concernent d'ailleurs qu'une faible partie du sol forestier, présentent une lacune que je dois relever tout d'abord. Cette lacune se rapporte au pâturage. Le code forestier et l'ordonnance réglementaire du $1^{\text {er }}$ août 1827 ne traitent du pâturage que lorsqu'il s'exerce dans l'intérieur des forêts où il est regardé comme secondaire, et rentrant dans la catégorie des produits accessoires. Mais puisqu'il est reconnu aujourd'hui que le pâturage constitue dans certains cas le principal et même le seul mode de jouissance des terrains qui se refusent à la culture agricole; que c'est à l'abus du pâturage surtout que l'on doit attribuer les ravages des torrents et la ruine de plusieurs de nos départements, il conviendrait d'ajouter aux lois forestières des dispositions spéciales pour régler le régime des pâturages, et le régime.qu'on appelle forestier devrait s'appeler aussi pastoral. Depuis la promulgation de la loi sur 
le gazonnement, ces dispositions spéciales et l'extension qui s'ensuivrait pour les attributions de l'administration forestière, sont devenues indispensables. II y a du reste longtemps que le besoin s'en fait sentir : N'a-t-on pas dû soumettre au régime forestier des terrains déboisés et livrés au parcours, parce qu'il n'était pas possible de les soustraire autrement à une dégradation complète? On a violé ainsi l'article 90 du code qui n'exige la soumission au régime forestier que des forèts susceptibles d'une exploitation régulière, et il en est résulté toutes sortes de difficultés entre les communes et l'administration. Il ne faut pas que ces choses se renouvellent, et la création légale d'un régime pastoral les empêchera de se renouveler. Je voudrais plus encore : je voudrais que le régime forestier lui-même n'impliquât pas la culture permanente et exclusive des bois; qu'il pût se concilier avec la culture agricole temporaire. S'il en était ainsi, les communes seraient encouragées à placer, d'ellesmêmes, sous la main de l'administration forestière, tous leurs biens communs dont la jouissance donne lieu à des désordres qu'elles sont impuissantes à réprimer'; mais j'aurai à revenir plus tard sur cette question, et je vais examiner de plus près, quoique sommairement, les attributions de l'administration forestière dans leurs rapports avec les biens de l'État, avec ceux des particuliers et avec ceux des communes. 
ARTICLE PREMIER.

ATTRIBUTIONS DE L'ADMINISTRATION FORESTIÈRE

dans LeURS RAPPorts avec les biexs de L'État.

Pour les biens de l'Étal, j'ai peu d'observations à faire : si on attribuait à l'administration forestière la gestion des pâturages et la mise en valeur des terres, peu nombreuses d'ailleurs, qui sont comprises dans ces biens; si on lui permettait en même temps d'appliquer la loi d'expropriation pour cause d'utilité publique aux acquisitions que réclamerait l'amélioration de la propriété, il n'y aurait rien à ajouter à l'autorité dont elle est déjà investie. N'est-il pas bizarre que la loi d'expropriation pour cause d'utilité publique soit applicable à l'achat d'un terrain nécessaire à la construction d'un chemin vicinal, et ne le soit point à celui d"un terrain nécessaire à la construction d'une route forestière? Est-ce que cette route n'a pas un caractère d'intérêt général plus prononcé, que le chemin vicinal? Les anomalies de ce genre pouvaient se comprendre lorsqu'on considérait l'État propriétaire d'immeubles comme un simple particulier, ayant des intérêts distincts de l'intérêt public. Mais cette notion de l'État s'est modifiée : l'État, c'est toút le monde, et rien de ce qui lui appartient ne peut être assimilé à unepropriété privée. Quand il fait une route pour faciliter la vidange d'une de ses forêts, il poursuit un but d'intérêt général, et 
la plus-value que cette route donne au prix des coupes, entre dans la caisse du trésor et profite à tout le monde. Il est à remarquer, au surplus, qu'à part ce point relatif à l'expropriation pour cause d'utilité publique, les priviléges accordés par la loi à l'administration forestière pour les forêts domaniales sont plutôt excessifs qu'insuffisants. Ils sont excessifs par exemple en ce qui concerne d'abord la délimitation. attendu qu'en déclarant que la délimitation est définitive, si au bout d'un an, à partir du dépôt du procèsverbal, aucune opposition n'a été faite de la part du propriétaire, l'article 12 du code forestier a privé celui-ci du bénéfice de la loi civile sur les péremptions et les prescriptions. Ils le sont également ensuite en ce qui concerne l'extinction des droits d'usage en bois : on peut dire que la loi forestière, à ce sujet est presque spoliatrice, puisqu'elle a pour conséquence, en définitive, et qucl que soit l'esprit de libéralité par lequel l'administration a essayé d'en corriger la rigueur, elle a pour conséquence de diminuer dans une forte proportion la jouissance de l'usager. L'équité voudrait certainement qu'on lui abandonnât en cantonnement une partie de forêt susceptible de fournir un produit égal au chiffre de la délivrance usagère. Elle le voudrait d'autant plus qu'il est de principe que cette délivrance ne doit jamais dépasser les besoins réels de la partie prenante; et l'équité est méconnue, par conséquent, lorsque le revenu que l'usager peut retirer de la propriété qu'on lui a concédée en échange de ses droits, descend au- 
dessous de ces besoins. Puisque, comme je le disais plus haut, l'Ĺtat c'est tout le monde, il ne saurait séparer, en matière d'usages, son intérêt de celui dès usagers. Nuire à l'un, c'est nuire à l'autre. L'extinction des droits d'usage est, assure-t-on, nécessaire, parce que ces droits s'opposent à l'amélioration des forêts. Je ne vois pas trop en quoi, car toutes les fois que des usagers ont voulu empêcher l'administration de faire dans les forêts grevées, les travaux d'aménagement ou autres qu'elle jugeait utiles, ils ont été déboutés de leurs prétentions. L'extinction des droits d'usage ne serait légitime que dans le cas où il n'y aurait pas d'autre moyen d'arrêter les usages à feux croissants, et d'empêcher qu'ils ne dévorent tous les produits des forêls; mais, dans ce cas, on pourrait au moins laisser à l'usager le choix entre l'extinction des droits ou leur limitation. Que l'on allègue que le service des droits d'usage est gênant pour l'administration; soit, c'est incontestable, mais l'administralion n'a point été instituée pour né faire que les choses qui ne troublent pas sa quiétude, et la gêne ne devrait pas l'effrayer. Le respect des usages, sauf à en concilier l'exercice avec les droits du propriétaire et avec les exigences d'une bonne exploitation, aurait cet avantage de maintenir les liens qui, rattachent à la conservation du sol forestier de nombreuses populations; tandis que, par l'effet du cantomnement, on désintéresse ces populations pour toute la partie de forêts dont elles ne jouissent plus, et, ce qui est plus grave encore, on compromet 
l'avenir de la partie qu'on leur abandonnc, en la soumettant à tous les dangers qui consistent dans les besoins incessants, l'inintelligence et l'impréroyance des municipalités. Convenons qu'il y a, dans la manière dont l'État remplit ses devoirs vis-ì-vis des communes, d'étranges contradictions : d'un côté, il a recours à toutes sortes de précautions pourles empêcher de dissiper leur capital, et, s'il se plaint de quelque chose, c'est de n'avoir pas assez d'autorité pour cela. D'un autre côté, quand il tient la source de leur revenu dans ses mains; quand rien ne le contrarie, ne l'entrave pour en régler la jouissance, et il en est ainsi relativement aux droits d'usage, il se dépouille de son autorité souveraine pour livrer le capital représentatif de ces droits à tous les hasards, à toutes les éventualités auxquels sont exposées les propriétés communales ordinaires dont la tutelle administrative cst la seule protection.

ARTICLE II.

ATTRIBUTIONS DE L'ADMINISTRATION FORESTIẺRE

DANS LEURS RAPPORTS AVEC LES BIENS DES PARTIGULIERS.

Sauf les pâturages d'une grande étendue, qui sont régis par l'administration des domaines, l'administration forestière est donc investie d'une autorité légale à peu près suffisante, pour améliorer le domaine forestier et pastoral qui appartient à l'État. Quelle 
est son autorité sur les propriétés de même nature que possèdent les particuliers? Elle est pour ainsi dire nulle. Sous l'empire de la fameuse ordonnance de 1669, les bois des particuliers étaient soumis à des règles d'exploitation qui avaient pour but d'en assurer la conservation et d'y favoriser la production d'une certaine quantité de pièces de fortes dimensions. Aujourd'hui, à part le défrichement que le gouvernement s'est réservé le droit d'interdire dans quelques circonstances exceptionnelles, les particuliers peuvent faire de leurs bois et de leurs pâturages ce que bon leur semble, et il serait probablement impossible de leur reprendre la liberté qu'on leur a rendue sous ce rapport. Cependant, l'expérience a prouvé que la simple prohibition du défrichement ne suffit pas à conjurer la destruction des bois, lorsque ces bois ont plus de valeur, plus de prix par le pâturage qu'ils fournissent aux bestiaux, que par les produits ligneux, et c'est ce qui explique cette vaste étendue de rapailles, de friches et de terrains vagues que les particuliers possèdent dans les montagnes du midi de la France ${ }^{1}$. L'expérience a prouvé aussi que les pâturages, à leur tour, s'anéantissent si on en fait abus, surtout lorsqu'ils sont situés en pente rapide;

1. Les personnes qui douteraient de l'inefficacité des lois à ce sujet n'ont qu'à lire le très-remarquable livre de M. de Ribbe sur la Provence au point de vue des bois et des torrents. Le parlement de Provence a multiplié les défenses et les peines. Les défriclıements et les écobuages n'en ont pas moins marché avec une rapidité désespérante. 
mais, pour réprimer cet abus, l'administration est tout à fait impuissante.

On commettrait donc la plus. grande des imprudences, si on laissait entre les mains des particuliers les bois et les pâturages qu'ils possèdent, dans l'intérieur du périmètre où il aurait été reconnu que l'intérêt général exige la conservation de ces bois et de ces pâturages. Il n'y a qu'une manière d'assurer cette conservation : c'est que l'État s'en charge, et, à cet effet, exproprie les particuliers pour. cause d'utilité publique. Mais puisque ces derniers sont incapables de, garder leurs terrains en nature de bois et de pâturage, lorsque leur intérêt du moment est de les livrer sans modération aux bestiaux, encore plus le sont-ils de mettre des bois ou des prés là où n'y en a pas, et j'en conclus que le gouvernement devrait encore acquérir tous les terrains des particuliers dont la dégradation serait préjudiciable à l'intérêt général du pays, et dont la mise en valeur serait au-dessus des forces de l'initiative individuelle.

Les lois sur le reboisement et le gazonnement des mońtagnes ont prévu que l'exécution des travaux par l'État, était en effet le meilleur moyen d'arriver à la consolidation et à la mise en valeur des terrains appartenant aux particuliers; malheureusement, elles ont admis que ces terrains, après avoir été consolidés et mis en valeur, pourraient être réclamés par leurs anciens propriétaires, moyennant l'abandon d'un quart ou la moitié de leur étendue, et c'est là une chose 
fort regrettable, ear elle expose l'État à perdre un quart ou la moitié de la dépense qu'il aura faite.

Ces lois ont aussi autorisé des subventions et des primes pour les reboisements à faire en dehors du périmètre d'utilité publique. C'est là une faute aussi grande selon moi que la précédente, comme j'essayerai de le prouver plus tard, quand j'aurai à traiter la même question pour les bois des communes.

Quant aux bois des particuliers qu'il n'y aurait pas lieu de soumettre au régime forestier et pastoral dans toute sa rigueur, ils seraient remis à la libre disposition des propriétaires, à moins que leur existence ne se liât à des intérêts locaux d'une certaine importance, auquel cas ils seraient maintenus, 'après enquête, sous l'empire de la prohibition du défrichement, et on en formerait un cadastre spécial ${ }^{1}$. Mais pour dédommager les propriétaires de cette servitude, l'État devrait leur accorder une indemnité et il en trouverait aisément les moyens, soit en supprimant les droits d'octroi qui grèvent si lourdement les

1. Toutes les lois sur le défrichement seront illusoires tant que ce cadastre ne sera pas établi. Je crois l'avoir prouvé dans la note sur le défrichement que j'ai présentée à la Société forestière en 1859. Mais, m'objectera-t-on peut-être, à quoi servira ce cadastre spécial s'il est vrai, comme vous l'avez dit, qu'il n'y a pas de loi, pas de contrainte qui puisse conjurer la ruine d'un bois, quand ce bois a plus de valeur par le pâturage que par les produits ligneux? - J'ai dit cela et j'y persiste, mais il y a heureusement encore beaucoup de bois qui ont plus de valeur par les produits ligneux que par le pâturage, et pour ceux-là la défense de défricher sera très-efficace, à moins que l'on n'ait affaire à des propriétaires dépourvus de raison. 
produits ligneux dans certainès villes, soit en diminuant l'impôt foncier qui est généralement plus élevé pour les bois que pour les autres immeubles, soit en concourant plus énergiquement à la poursuite et à la répression des délits commis dans ces bois, soit enfin en favorisant par des subventions la création de chemins forestiers. Le gouvernement a du reste fait un premier pas dans le sens que j'indique ici par la loi du 27 juillet 1870, sur la région des. Maures et de l'Estérel, en Provence, loi dont le pays est redevable à l'initiative du directeur général actuel des forêts, M. Faré. Par cette loi, les gardes forestiers de l'État ont été associés à la poursuite des délits et contraventions commis dans les bois des particuliers; l'État 's'est chargé de la moitié des dépenses qu'entraînera la construction d'un réseau de routes destiné à améliorer la situation de ces bois; et, chose digne de remarque encore plus que les précédentes, ces routes sont appelées à jouir de tous les priviléges dont jouissent les chemins vicinaux d'intétérêt commun. Ainsi voilà enfin le principe de la protection spéciale que l'État doit aux propriétés boisées des particuliers, consacré et sanctionné par une loi. Il ne s'agit plus que d'en faire d'autres dans le même but. 
ARTICLE III.

ATTRIBUTIONS DE L'ADMINISTRATION FORESTIÈRE

DANS LEURS RAPPORTS AVEC LES BIENS DES COMMUNES.

Si on devait juger du mérite des lois et des règlements forestiers par l'état dans lequel sont les bois communaux, on en aurait une bien faible idée, car il n'y a pas une branche du service qui, pour ces bois, ne soit en souffrance.

Délimilation et Bornage. — Sur plus de 1,900,000 hectares de forêts que possèdent les communes, y en a-t-il seulement la moitié qui aient été délimitées et abornées régulièrement? - Je ne le crois pas.

Surveillance. - Sauf quelques forêts de l'est et du centre, la surveillance de ces forêts n'est assurée nulle part, et pour s'en convaincre, il n'y a qu'à voir à combien s'élève en moyenne le traitement d'un garde forestier communal. Dans les 22 conservations où il est le plus fort, il ne dépasse pas 400 francs. Dans le Midi et en Corse, il descend à des chiffres dérisoires : $150 \mathrm{fr}$., $100 \mathrm{fr}$. et all-dessous, et encore n'est-il pas toujours payé. On sait que ce sont les conseils municipaux qui fixent ce traitement, sauf l'approbation du préfet. Quand ils ne veulent pas des gardes nommés par les préfets sur la proposition des conservateurs, ils ont un moyen bien simple de se débarrasser de ces gardes, c'est de supprimer leur traitement. 
Quarts en réserve. - Malgré les efforts de l'administration forestière, les quarts en réserve des forêts communales sur lesquels on comptait pour élever des arbres de fortes dimensions, des futaies, ont été généralement convertis en taillis que l'on exploite au même âge que les coupes ordinaires ${ }^{1}$. Les épargnes accumulées par les siècles, ont été ainsi dissipées au grand détriment des générations futures. Puis sont venues les délivrances par anticipation; il y a des départements, celui de la HauteSaône entre autres où, par l'effet de cette anticipation, les taillis ne présentent plus les éléments d'un balivage convenable.

Aménagemenı. - Toutes les tentatives faites pour soumettre les forêts communales à des aménảgements réparateurs n'ont abouti, la plupart du temps, qu'à de fàchenx résultats, parce que les municipalités ont refusé les ressources nécessaires pour les rendre efficaces. Expliquons cela : rien ne semble plus utile assurément que de remplacer par exemple la méthode jardinatoire, quand d'ailleurs le climat, l'altitude, la configuration du sol, le permettent, par la méthode du réensemencement naturel et des éclaircies périodiques. La conséquence immédiate d'une transformation de ce genre est une augmentation sensible de revenu, puisque après avoir partagé la forêt en un certain nombre d'affectations correspon-

4. Voir pour plus de détails la note sur les quarts en réserve que j’ai publiẻe dans les Annales forestières en 1859. 
dantes à un même nombre de périodes de la révolution, au lieu de se borner comme on le faisait auparavant à jardiner sur toute la surface, on enlève pendant la première période tout le matériel de la première affectation, tandis qu'on continue le jardinage dans les autres. Les communes acceptent dès lors avec empressement les projets de conversion dans ce sens; mais leur demande-t-on quelques fonds pour préparer, favoriser et compléter le repeuplement naturel dans l'affectation en cours de régénération, elles s'y refusent. Le sol se durcit, le repeuplement devient impossible, les arbres espacés par la coupe d'ensemencement sèchent en cime, ou sont renversés par le vent. L'aménagement au lieu d'améliorer la forêt en compromet l'existence; au lieu d'être bienfaisant il est désastreux.

S'agit-il d'un taillis composé à transformer en futaie pleine? il y a également tout d'abord augmentation de revenu, lorsque la transformation n'est pas précédée d'une révolution préparatoire et lorsqu'elle est graduelle, et les communes l'accueillent encore avec grand plaisir; mais elles ne veulent pas entendre parler des repeuplements artificiels que ces sortes de conversions rendent pourtant indispensables sous peine de manquer leur but; et, au lieu d'être transformée en futaie pleine, la forèt est transformée en taillis simple. .

Coupes, produits principaux et accessoires. - II y a dans les forêts des exploitatioñs qui, souvent onéreuses pour le moment, sont très-avantageuses pour 
l'avenir; ce sont les nettoiements par exemple: on les néglige complétement dans les forêts communalés. Quant aux coupes principales, la distribution des produits en nature entre les habitants, ce qu'on appelle l'affouage, leur ôte une grande partie de leur valeur et l'administration forestière n'y peut absolument rien.

C'est un fait que partout les communes abusent des menus produits : le pâturage, le panage, l'enlèvement des feuilles, et que l'appauvrissement complet de certaines forêts en a été la suite.

Joyens de vidange. - C'est un fait aussi que les moyens de vidange sont encore dans les forêts communales à l'état primitif, et d'ailleurs, il faut le reconnaître, il y a peu de communes qui puissent y consacrer les ressources pécuniaires que leur amélioration réclamerait. Tandis que l'on compte plus de 12,000 kilomètres de routes pour les forêts domaniales qui ne contiennent pas un million d'hectares, on n'en compte que 7,000 à peine pour plus de $1,500,000$ hectares de forêts communales situées dans les départements les plus riches, et encorę peut-on assurer que ces 7,000 kilomètres de routes sont à peine carrossables! or il en faudrait au moins 25 à 30 milles kilomètres. Dans les Pyrénées, dans les Alpes, en Corse, il y a un grand nombre de forêts communales qui ne rapportent rien et qui rapporteraient beaucoup si on y dépensait quelques milliers de francs pour des chemins.

Voilà donc à quel point les forêts des communes 
sont abandonnées, sauf un petit nombre d'exceptions. Que dirai-je maintenant de celles de leurs propriétés qui sont exclusivement affectées au pâturage, et que l'administration forestière n'a point à surveiller ? C'est en y accumulant outre mesure les bestiaux qu'on y a favorisé, autant au moins que par le déboisement, les ravages des torrents. Une partie de la Provence a été amenée à un tel degré de paurreté que les chèvres même ne trouvent plus à y vivre ${ }^{1}$. Rien de plus triste à voir que ces collines calcinées par un soleil ardent, où ne poussent plus que de rares cépées rabougries qu'assiégent constamment une multitude de chèvres affamées. Ces terrains ne sont pas cependant tout à fait improductifs : de temps en temps, on en retourne la couche superficielle, on la brûle et on sème ensuite des céréales; mais cette ressource, elle aussi, tend à disparaître, parce qu'elle aurait besoin comme les autres d'être ménagée et qu'elle ne l'est pas. On devrait, quand une ou deux récoltes ont été obtenies, laisser le sol en repos pendant douze à quinze ans avant de lui en demander une troisième. On devrait surtout n'écobuer que les parties plates ou en pente peu rapide. Or les habitants, si rien ne les en empêche, poursuivent la culture jusqu'à ce qu'elle ne leur rapporte plus de quoi payer leur peine, et alors, ce n'est pas seulement quinze ans qu'il faut

1. Lire à ce sujet l'instructif et saisissant ouvrage déjà cité do M. de Ribbe: La Provence au point de vue des bois, des torrenits et des inondations, 1857. (Guillaumin et Gis libraires.) 
à ce sol pour produire encore quelque chose; c'est un temps indéterminé, la durée souvent de plusieurs générations. En outre, on écobue les parties en pente rapide comme les autres, et, alors, l'épuisement du sol peut devenir irrémédiable. Les municipalités le savent bien; aussi leur est-il arrivé quelquefois, dans l'impuissance où elles sont de mettre un frein à l'avidité et à l'imprévoyance de leurs administrés, de demander la soumission au régime forestier des terrains en question, et voilà comment l'administration forestière a été chargée contrairement aux dispositions de la loi, de gérer des biens qui ne sont nullement susceptibles d'exploitation forestière régulière. La mesure n'en eût pas moins été bonne cependant si, en même temps qu'on confiait à cette administration des terrains auxquels le régime forestier n'était pas immédiatement applicable, on lui avait laissé toute latitude pour en appliquer un autre. Malheureusement cela n'a pas eu lieu, et les communes propriétaires ont protesté, alléguant qu'en soumettant leurs biens au régime forestier, elles n'avaient pas entendu se priver à tout jamais d'en retirer aucun produit.

On voit en définitive que les communes sont en général tout à fait inhabiles à administrer avec sagesse, les biens sur lesquels on leur a laissé toute autorité; et si elles n'ont pas non plus beaucoup à'se féliciter de la situation dans laquelle sont ceux, dont la gestion a été confiée à l'administration forestière, ce n'est pas la faute de cette administration, c'est la faute de la loi et des règlements qui ne lui ont pas 
donné la faculté, soit de satisfaire aux désirs légitimes des municipalités, soit de lutter contre leurs exigences et leur mauvais vouloir.

Quand les agents forestiers s'opposent à ce que les habitants d'une commune cultivent, même temporairement, des terrains soumis au régime forestier, bien que ces terrains ne soient pas boisés, ils sont certainement dans leur droit et dans leur devoir; ce n'est pas eux qui ont tort, c'est la loi. Il faudrait que la loi fùt modifiée et que l'administration forestière eût toute liberté pour régler la culture des propriétés qui auraient été placées sous sa direction, de la manière la plus avantageuse pour la commune propriétaire, eu égard à ses besoins et à ses ressources ${ }^{1}$. Il faudrait aussi ranger dans les attributions de cette-administration, comme je l'ai fait observer déjà à propos des biens domaniaux, l'exploitation des pâturages communaux ${ }^{2}$. Mais cela ne suffrait pas si l'action de l'administration sur ces

1. Les cultures temporaires sont en général détestables. On peut les ranger au nombre des plus grands fléaux de la Provence. Cependant on rencontre, dans les terrains soumis au régime forestier, des rides assez étendus qui ne sont point en pente et que les communes ne peuvent reboiser artificiellement, parce qu'elles n'ont pas les ressources pécuniaires qu'exigerait cette opération. En attendant que l'on soit en mesure de rendre ces vides à la culture forestière, pourquoi ne permettrait-on pas aux habitants de leur demander de temps en lemps une ou deux récoltes de céréales? Quel mal y verrait-on? Ici comme ailleurs, il ne saurait y avoir de règle sans exception, ou plutòt il faut des exceptions pour confirmer la règle.

2. Il en était ainsi sous l'empire de l'ordonnance de 1669. 
biens, n'était pas mieux assurée que celle qu'elle a exercée jusqu'à présent sur les bois susceptibles d'exploitation régulière; et nous avons à chercher maintenant comment on pourrait rendre sa tutelle plus efficace.

Pourquoi cette tutelle n'a-t-elle pas produit de meilleurs résultats? Est-ce à cause de l'imperfẹclion de la loi ? - Oui et non. - Oui, parce que la loi exige que toute les mesures que comporte la gestion des forêts communales, soient soumises aux délibérations des municipalités; parce qu'elle donne trop d'influence aux préfets sur la suite dont ces délibérations pourraient être susceptibles; parce qu'elle leur confère le pouvoir de nommer les gardes et de fixer leurs traitements; parce qu'elle ne permetpas à l'administration forestière de vaincre la résistance des communes, lorsque cette résistance s'appuie sur la pauvreté vraie ou prétendue des caisses municipales. Non, parce qu'en somme, en définitive, l'autorité supérieure a le droit de décider en dernier ressort sur presque toutes les questions forestières communales, et de porter d'office aux budgets communaux les dépenses nécessaires. L'autorité supérieure n'use pas de ce droit; elle n'écoute pas assez les avis de l'administration forestière. Telle est la raison dominante de la dégradation dans laquelle est tombée la propriété forestière communale.

- Jusqu'à présent, aucun gouvernement n'a été assez fort pour mettre un frein à l'avidité des communes -relativement aux coupes exlraordinaires et aux 
feuilles mortes; pour renfermer le parcours des bestiaux dans les limites raisonnables; pour imposer d'office les dépenses indispensables à l'exécution des aménagements et des travaux les plus urgents. Aucun gouvernement n'a été assez fort, même pour assurer aux gardes un traitement suffisant. Il aurait fallu braver le mécontentement des électeurs; aucun gouvernement ne l'a osé. Que de fois n'a-t-on pas vu des conservateurs désavoués, presque disgraciés, parce que, indignés du mal qui se développait sous leurs yeux, ils avaient fait quelques tentatives pour y porter remède. Blâmés par l'inspection des finances, à cause de leur indulgence, ils l'étaient également par le ministère, à cause de leur sévérité.

Que de fois aussi n'a-t-on pas vu les mêmes hommes qui, comme membres des conseils départementaux, avaient, par leurs vœux, engagé l'administration à plus de rigueur dans l'application des règlements forestiers, être les premiers, comme maires ou députés, à se plaindre des mesures qu'ils avaient provoquées.

Ces contradictions n'ont rien de surprenant. Elles sont presque inévitables devant deux intérêts : l'intérêt de ce qui meurl et l'intérêt de ce qui ne meurt pas, l'intérêt du présent et celui de l'avenir, dont la conciliation est, quoi qu'on dise, très-difficile. En théorie, c'est à l'intérêt de l'avenir que l'on donne ordinairement raison. Dans la pratique, c'est celui du moment qui l'emporte. Les préfets, les conseils généraux, lorsqu'ils sont appelés à envisager la si- 
tuation dans son ensemble et dans ses conséquences désastreuses pour les générations futures, se récrient dans des rapports, dans des délibérations très-énergiques, contre les prétentions exagérées des populations forestières. Le lendemain, quand ils se retrouvent aux prises avec les détails, avẹc les exigences locales, les nécessités parfois bien pressantes du moment, et avec le désir de conserver leur influence, leur popularité, ils oublient vite leurs belles protestations de la veille, et ils accusent eux-mêmes l'administration forestière de tracasserie et même de tyrannie, tandis qu'elle ne veut cependant qu'empêcher la jouissance des communes de devenir trop abusive.

L'administration forestière, on ne le sait que trop, est peu populaire dans une partie de la France, et rien ne prouve mieux combien le régime qu'exige la conservation des forêts est odieux aux communes. En effet, si une administration est placée vis-à-vis de ceux qui lui sont soumis, dans des conditions d'impartialité, c'est bien celle-là. La gestion qu'elle exerce dans les forêts communales est entièrement désintéressée, non-seulement en ce qui concerne l'État, puisqu'il ne prélève pas ce qu'il faudrait pour le dédommager des frais qu'elle lui impose, mais aussi en ce qui concerne les agents, puisqu'elle ne leur procure aucune indemnité, aucun bénéfice. II est donc nécessaire que ces agents soient fortement pénétrés de leurs devoirs et désireux de faire le bien, pour qu'ils puissent résister aux obsessions de tout 
genre qui les assiégent, par suite de l'égoïsme de ceux dontils administrent les propriétés. Il leur serait facile de se faire aimer autant qu'on les déteste : il leur suffirait pour cela d'ouvrir une libre carrière à tous les appétits des conseils municipaux; mais leur conscience en souffrirait. Tel est le sentiment qui les retient. Il n'y en a pas d'autre.

Il n'en est pas moins vrai que les forêts communales disparaissent insensiblement, poussées à leur perte par des forces qu'on croirait fatales. Elles s'en vont notamment par trois débouchés qui s'élargissent chaque jour de plus en plus : le pâturage, l'enlèvement des feuilles et les coupes extraordinaires. Aux yeux des conseils municipaux, trop souvent soutenus par les préfets, il y a toujours place pour les moutons dans les forêts, il y a toujours trop d'humus, et tant qu'il y reste un arbre, il y a matière à une coupe extraordinaire ${ }^{1}$.

A tune telle situation, je ne vois qu'un remède

1. Ces coupes, le bon sens l'indique, ne devraient être prises que sur l'épargne, sur la réserve, sur l'excédant du matériel superficiel nécessaire pour assurer un produit égal au plus grand accroissement moyen; mais il n'en est point ainsi : les communes ne font pas de différence entre le plus grand accroissement moyen et un accroissement moyen quelconque, et roici comment elles raisonnent: la forêt peut rapporter $\boldsymbol{X}$ mètres cubes, pour que la coupe annuelle ne dépasse pas le chiffre de l'accroissement moyen quel qu'il soit. Sur ces X mètres cubes, prélevons les $3 / 4$ pour les coupes ordinaires; le reste est disponible pour les coupes extraordinaires. A ce comple-là, quel que soit le matériel sur pied, une coupe extraordinaire est toujours possible, malgré l'appauvrissement de la forêt. 
infaillible : l'acquisition par l'État de toutes les forêts des communes et des établissements publics.

Une pareille proposition excitera l'étonnement; cependant on va voir que l'acquisition proposée, faite d'une certaine manière, constituerait-une opération facile et peu onéreuse.

Si l'Etat venait aujourd'hui proposer aux communes de leur acheter leurs forêts à condition de leur conserver le droit au pâturage et de leur assurer, en outre $: 1^{\circ}$ une rénte sur le grand livre égale au produit en argent, largement évalué, qu'elles retirent des coupes de ces forêts; $2^{\circ}$ une délivrance en nature égale au volume des.coupes destinées à. l'affouage; une telle offre n'aurait-elle pas de grandes chances d'être acceptée? Pour moi, je n'en doute point. Comment les communes n'accueilteraientelles pas avec empressement une transaction qui, tout en leur permettant de jouir du même revenu, les affranchirait des charges considérables, impôts, frais de garde et de gestion, qui grèvent leur propriété et qui souvent la rendent onéreuse ${ }^{1}$. Pour les biens de mainmorte, pour les biens inaliénables, le prix qui s'attache au titre de propriétaire est une pure fiction, et le revenu, le produit de ces biens, est la seule chose qui mérite d'être prise en considération. Puisque sous ce rapport les communes ver-

1. M. Hun, ancien conservateur des forêts à Grenoble, a établi dans une brochure très-instructive sur les inondations (1850) que le revenu moven des bois communaux dans les Alpes est à peine de 1 franc par bectare. 
raient, par suite de la cession de leur's forêts à l'État, leur situation s'améliorer, on ne s'expliquerait pas vraiment qu'elles hésitassent à consentir cette cession. Mais l'État, de son côté, n'aurait pas fait une mauvaise affaire en acquérant, au prix que j'ai indiqué, les forêts communales; car, en admettant que cette acquisition lui imposât pour le moment quelques sacrifices, il est certain qu'elle le dédommagerait amplement plus tard de ces sacrifices par la plus-value, qu'au moyen d'une intelligente administration, il ne pourrait manquer de donner aux forêts qu'il aurait ajoutées à son domaine.

Voilà donc par quelle opération simple et rationnelle on remédierait d'un seul coup à tous les vices que présente la gestion des forêts communales, vices qui se traduisent par des pertes incalculables, nonseulement pour les communes propriétaires, mais pour la société tout entière; et cette opération ne coûterait pas beaucoup à l'État, puisque la vente des coupes dans les forêts qu'il aurait acquises, lui procurerait les ressources nécessaires pour payer les rentes dont il aurait augmenté sa dette. Le surcroît de dépense qui lui incomberait consisterait dans les frais de gestion, qui seraient désormais entièrement à son compte : quelques millions au plus! mais qu'est-ce que cela auprès de la richesse immobilière qu'il créerait en appliquant aux deux millions d'hectares, dont se compose le domaine forestier communal, les améliorations et les méthodes 
d'exploitation qu'il applique à ses propres forêts ${ }^{1}$ ! Malheureusement, un parti aussi radical rencontrerait, j'en ai peur, malgré tous ses avantages, un obstacle invincible dans les préjugés de nos hommes d'État et dans leur manière de comprendie l'économie des dépenses. Aussi, me bornerai-je à en demander l'adoption pour les forêts situées dans le périmètre dont la conservation aurait été déclarée d'utilité publique 2 . Ici, l'expropriation par l'État est indispensable; car on aura beau chercher d'autres expédients, inscrire dans la loi des prérogatives plus grandes pour l'administration forestière; ces prérogatives ne constitueront jamais des garanties tout à fait suffisantes pour la conservation des forêts, tant que les communes auront une action sur la gestion de cette partie de leurs biens; et cette action, comment pourrait-on la leur enlever absolument, puisqu'elle est inhérente au droit de propriété?

Cependant, n'y aurait-il rien à faire de mieux que ce qui existe, dans l'intérêt des forêts que l'État

1. Les produits en argent des forèts communales ne dépassent guére 20 millions par an. Il y aurait donc 20 millions de plus à inscrire au grand-livre. Il ne s'agit pas, on le voit, d'une grosse affaire.

2. Cn écrivain distingué, bien connu par ses travaux en économio forestière et en économie politique, M. J. Clavè, a publié dans la livraison du $1^{\text {er }}$ février 1869 de la Revue des Deux Mondes, un article sur le reboisement des montagnes, qui mérite d'être lu comme tout ce qu'il fait, et qui conclut aussi à l'exproprialion pour cause d'utilité publique de toutes les forêts dont la conservation serait d'intérêt général. 
n'aurait point acquises, et faudrait-il les abandonner à toutes les influences qui les ont déjà si sensiblement appauvries?

Je vais indiquer à ce sujet quelques mesures, sans me dissimuler qu'elles ne seraient que palliatives :

La première, la principale de ces mesures, serait de diminuer sinon de supprimer l'autorité des préfets dans la gestion des forêts communales, et de leur interdire toute ingérence dans les discussions d'économie forestière ${ }^{1}$. On devrait ensuite ne soumettre aux délibérations des communes propriétaires que les questions qui pourraient être résolues dans un sens ou dans un autre, sans que la conservation de la propriété en fût compromise; et inscrire à leurs budgets, comme obligatoires, toutes les dépenses nécessaires pour l'entretien des travaux faits et pour l'exécution des travaux à faire, en vertu de décisions régulièrement prises. N'est-il pas déplorable qu'un conseil municipal qui a voté une dépense puisse, l'année suivante, revenir sur sa délibération et annuler ce qu'il a fait; qu'après avoir donné son approbation à un projet d'aménagement, il puisse la retirer ou mettre l'administration des forêts dans l'impossibilité d'appliquer le projet, en lui refusant les fonds

1. La loi départementale du 10 aoùt 1871 n'a rien changé aux attributions des préfets en matière forestière; mais elle a appelé les conseils généraux à délibérer sur les questions d'aménagement, et il est à craindre qu'elle n'ait ainsi ajouté une grave difficulté à celles que rencontre déjà la solution de ces questions. 
indispensables? C'est pourtant ce qui arrive tous les jours.

On devrait, en oulre, faire nommer les gardes communaux par l'administration forestière, qui fixerait également leur salaire; car, sans cela, ces gardes continueront d'être les très-humbles et obéissants servileurs de IIM. les maires, et de faire toutes sortes de métiers, excepté celui pour lequel ils ont été institués.

Mais il est évident que pour que la réforme que j'indique ne fût pas plus apparente que réelle, il fau-. drait ne pas se borner à décider que l'administration forestière aura désormais toute latitude pour gérer les forêts communales de la manière qu'elle jugera le plus convenable; il faudrait, en mème temps, lui fournir les ressources pécuniaires dont elle aurait besoin à cet effet.

Comment les lui fournira-t-on? C'est le problème capital à résoudre.

Pour moi, je voudrais que l'État fit toutes les avances de fonds que réclameraient la gestion, la conservation et l'aunélioration des forêts communales, sauf à se rembourser sur le produit de la vente des coupes, à défaut d'autres moyens '.

1. Arouons que lorsque "les communes se plaiguent de la tutelle de l'État, ce n'est pas toujours sans raison; car c'est unsingulier luteur, convenons-en, que celui qui laisse son pupille dans la misère, quand il lui suffirait pour l'en tirer de lui tendre une main secourable, sans d'ailleurs courir aucun risque. Parmi beaucoup d'exemples que je pourrais citer de l'insouciance de 


\section{Parmi ces avances, il y en aurait d'obligatoires, il y en aurait de facultatives.}

l'État, en ce qui concerne les forèts communales, j'en choisis un qui est caractéristique.

Il s'agit d'une commune, la commune de Serragio, qui possède en Corse une forêt de 1,600 hectares environ.

Il a été constaté par l'aménagement qui vient d'être fait de cette forêt :

$1^{\circ} Q u^{\prime} e l l e$ contient un matériel considérable en bois exploitables;

$2^{\circ}$ Que faute de route, le mètre cube n'y a presque pas do valeur;

$3^{\circ}$ Qu'il faudrait dépenser 40,000 francs pour construire une route de 6 à 7 kilomètres;

$4^{\circ}$ Que celte route construite, le prix du mètre cube sur pied s'élèvera au moins à 10 francs, et qu'indépendamment d'une somme immédiatement réalisable de $1 \ddot{50} 0,000$ francs, la forêt pourra rapporter éternellement un revenu annuel de 7,000 francs.

Malheureusement, la commune de Serragio n'a pas 40,000 francs et elle ne sait où les prendre. Que propose-t-on alors? - On propose de vendre, dans les conditions présentes, une quantité d'arbres assez grande pour procurer à la commune cette somme de 40,000 francs. Au prix actuel, ce serait un volume énorme qu'il faudrait jeter bas, de sorte que si la proposition était adoptée, la forêt n'aurait de longtemps plus rien à fournir.

On dira peut-être que puisqu'il suffirait d'une dépense de 40,000 francs pour élever le prix du mètre cube de bois à $10 \mathrm{fr}$., il y aura des spéculateurs qui seront disposés à faire celte dépense et à tenir compte dans leurs offres de la plus-value qu'elle donnera au bois. Cette objection ne serait pas indifférente en France où il $\mathrm{y}$ a de grands capitaux, et où le commerce des bois est trèsconnu. Mais en Corse elle est sans valeur, parce que tout y manque, le capital et l'expérience du commerce; et si on procédait à la vente que désire la commune de Serragio, les résultats en seraient faciles à prévoir : on ne payerait pas le bois plus cher qu'on ne l'a payé jusqu'à présent; la forêt serait ruinée, et le produit de l'adjudication, au lieu de servir à la construction d'une 
Il y en aurait de remboursables intégralement, tandis que les autres pourraient ne l'être qu'en partie.

Seraient obligatoires les avances relatives: $1^{\circ} \mathrm{A}$ la gestion ordinaire, gestion qui comprendrait non-seulement les opérations prévues par l'article $107 \mathrm{du}$ Code forestier, mais encore la survèillance, la constatation des délits, et l'étude des aménagements ${ }^{1} ; 2^{\circ}$ à

rcute, derenue d'ailleurs pour longtemps inutile, serait employé à la construction d'une église.

Pourquoi l'État ne fait-il donc pas la route à ses frais, sauf à se rembourser sur le procuit de la première coupe? Il ne lui faudrait pas un an roar rentrer dans son argent, intérèt compris, s'il le voulait. Quand on le voit si ̨̧énéreux pour l'agriculture et pour l'industrie, n's-t-on pas le droit de s'étonner et de se plaindre du peu de souci qu'il semble prendre des in'érêts de la sỵlviculture. Avec le quart tout au plus des sacrifices qu'il s'est imposés pour encourager le crainage, il pourrait (ripler la production des forèts communales.

1. Je ne m'explique pas, je l'aroue, pourquoi les éludes d'arrénagement ne sont pas comprises parmi les opérations de conservation et de régie. L'aménaçement est la base indispensable de la gestion. Il n'y a pas d'opération importante qui soit possible sans lui. Faut-il instruire une demande de coupe estraordinaire, ou procéder au simple martelage d'une coupe ordinaire, il est nécessaire de se livrer à des reconnaissances, à ces calculs, à des travaux qui constituent ce qu'on appelle l'aménagement. N'est-il pas au moins bizarre que lorsque les coupes d'un taillis ne sont pas assises sur le terrain, on ne fasse rien payer à la commune propriétaire pour en esécuter chaque année l'arpentage, et que lorsque cette commune consent à ce que toutes les coupes soient arpentées en une fois définitivement, ce' qui revient à asseoir l'aménagement sur le terrain, on lui réclame les frais de l'opération. Ce que doivent supporter les communes, ce sont évidemment les frais extraordinaires de l'aménagement, tels que ceux qu'entral- 
l'entretien, aux assainissements, aux délimitations, à l'achèvement des travaux commencés, et en général, à l'exécution de tous les travaux nécessités par des décisions de l'autorité supérieure.

Seraient facultatives, les avances qui concerneraient les travaux extraordinaires de premier établissement, tels que les routes, les repeuplements d'une grande étendue.

Seraient remboursables intégralement les avances pour travaux de toute nature, neufs ou d'entretien, à moins que l'État n'y fût directement intéressé.

Pourraient n'être remboursées qu'en partie, les avances faites pour la gestion ordinaire, définie comme elle l'est ci-dessus, et voici la règle que je proposerais de suivre pour fixer la proportion dans laquelle le remboursement serait effectué :

Cette proportion serait plus ou moins forle, suivant que les produits principaux d'une forêt seraient plus ou moins élevés, par rapport aux frais de gestion : Ainsi, l'État prendrait par exemple à sa charge l'excédant desdits frais sur le quinzième du prix des produits principaux vendus ou délivrés, ou, ce qui reviendrait au même, il devrait faire face aux frais de gestion, sans que les communes fussent tenues à un rembourrsement qui dépasserait le quinzième de la valeur des produits principaux de leurs forêts.

Je ne pose, je ne défends ici qu'un principe, et

nent les expéditions des projets et des plans, les travaux de route, d'assainissement, de repeuplements artificiels, mais l'étude do l'aménagement devrait être à la charge de l'État. 
ce quinziè:ne des produits principaux en argent, qui me paraîtrait suffisant pour indemniser l'État de ses avances, pourrait ne pas l'être ou être excessif. C'est une question à examiner et que l'on résoudrait équitablement selon moi, si on limitait la répétition de l'État au rapport existant entre les frais de gestion et les produits principaux des forêts domaniales ou communales qui sont dans les meilleures conditions au point de vue de la production. Il est certain que ce rapport étant appliqué à toutes les forêts communales, l'État aurait à faire, pour l'ensemble de ces forêts, des frais qui ne lui seraient pas totalement remboursés ; mais n'oublions pas qu'en imposant aux communes de garder leur fortune sous la forme de bois exploitables à un cerlain âge, la société leur occasionne un préjudice notable. Il est possible de se faire une idée de ce préjudice par l'augmentation de revenu que se procureraient les communes, s'il leur était permis d'aliéner leurs forêts pour en placer le prix en rentes sur l'État. Cette opération aurait souvent pour résultat de doubler au moins leur revenu; de sorte que si elles pouvaient être assimilées à de simples particuliers, l'État, pour les dédommager des restrictions qu'il apporte à . la jouissance de leurs propriétés, leur devrait une indemnité annuelle égale au revenu actuel de ces propriétés. Je n'entends pas qu'on aille jusque-là, car dans l'intérêt même des communes, il convient que leur fortune soit placée de la manière la plus solide, dût-elle rapporter moins. Toujours est-il que 
ce n'est pas seulement pour leur bien, que c'est aussi pour le bien public, qu'on les oblige de conserver leurs forêts et de les soumettre à une certaine révolution. Toujours est-il que moins le revenu que donnent ces forêts est élevé, plus évidente et plus grande est la part de l'intérêt général dans leur conservation. Il est donc rigoureusement juste que la société tout entière contribue d'autant plus aux dépenses qu'entraîne la gestion des forêts communales, que celles-ci sont pécuniairement moins productives, et qu'il y aurait plus d'avantages pour les communes propriétaires à les aliéner. Et qu'on ne se récrie pas devant une telle proposition, car elle ne tend qu'à confirmer le principe qui a été adopté par le Code forestier en matière de gestion des forêts communales. Aujourd'hui déjà, pour cette gestion, les communes ne sont tenues qu'au versement, jusqu'à concurrence de $1 \mathrm{fr}$. par hectare, du vingtième des produits des coupes principales vendues ou délivrées, de sorte que cette gestion mal définie d'ailleurs et trop restreinte, est gratuite pour les communes dont les forêts ne donnent pas de produits principaux. Il s'agirait d'étendre et d'améliorer ce principe, en comprenant dans les actes de gestion la surveillance, les aménagements même, quand ils n'entraîneraient pas de dépenses extraordinaires; et en supprimant la limite de $1 \mathrm{fr}$. par hectare, qui favorise les communes dont les forêts sont très-productives ${ }^{1}$.

1. Les forêts communales rapportent aujourd'hui en produits 
ARTICLE IV.

CONCLUSIONS APPOYÉRS SUR LES LOIS

REI.ATIVES AC REBOISEMENT ET A GAZONNEYENT

DES YOXTAGXES.

Les diverses propositions que je viens de développer, les principes que je voudrais voir adopter, sont du reste en germe dans les lois sur le reboisement et le gazonnement des montagnes, lois que nous allons maintenant passer en revue:

Les lois sur le reboisement et le gazonnement visent deux catégories de travaur: les travaux obligatoires et les travaux facultatifs.

Les travaux sont obligatoires lorsqu'ils ont été déclarés d'utilité publique par un décret rendu en conseil d'État, après enquête de commodo et incommodo.

Les travaux sont facultatifs quand leur utilité n'est que secondaire et qu'il suffit, pour la constater, d'une décision du directeur général des forêts.

Les reboisements et les gazonnements obligatoires, ainsi que toutes les consolidations de terre qu'ils nécessitent au préalable, sont exécutés par l'État

principaux plus de $\mathbf{4 0}$ millions de francs, elles contiennent 2 millions d'heclares, et cependant le chiffre de leurs contributions pour les frais de régie n'est que de $1,300,000$ francs. Il faut donc qu'il $y$ ait des forèts où la limite de 1 franc par bectare empèche la contribution de s'élever jusqu'au 1/20 des produits. 
lorsque les propriétaires ne peuvent ou ne veulent s'en charger. L'administration forestière s'empare des terrains, en appliquant à ceux des particuliers la loi d'expropriation pour cause d'utilité publique, et en soumettant au régime forestier ceux des communes. Quand les travaux sont terminés, les particuliers ont le droit d'obtenir leur réintégration dans leurs propriétés, à la cliarge de restituer l'indemnité d'expropriation et le prix des travaux; mais ils peuvent s'exonérer du remboursement du prix des travaux en abandonnant la moitié ou le quart de leurs propriétés: la moitié quand il s'agit de terrains reboisés, le quart quand il s'agit de terrains gazonnés. Quant aux communes, elles peuvent également se soustraire à toute répétition de la part de l'État, en lui abandonnant la moitié ou le quart de leurs biens; mais elles conservent dans tous les cas le droit de pâturage dans les parties boisées devenues domaniales, et les pâturages dont elles retiennent la propriété restent soumis au régime forestier, chose dont je prie qu'on se souvienne.

Les lois en question prévoient en outre que des indemnités pour interruption de jouissance, pourront être allouées aux communes dont les propriétés auraient été mises en défens par suite des travaux à exécuter.

Les reboisements ou gazonnements facultatifs ne donnent lieu de la part de l'État qu'à des exemptions d'impôts, ì des primes el à des subventions en nature ou en argent, non remboursables sauf malversations. 
Telle est l'économie de ces lois. Je leur reproche :

$1^{\circ}$ De faire intervenir l'État par des moyens contiaires aux principes de l'économie politique, dans des travaux qui n'intéressent pas la société tout entière; qui devraient par conséquent être abandonnés complétement à l'initiative individuelle; et qui du reste seront éphémères si cette initiative, à elle seule, n'est pas capable de les entreprendre et de les mener à bonne fin.

$2^{\circ}$ De compromettre le résultat des sacrifices qu'elles imposent au trésor public, pour les travaux d'utilité générale, en autorisant la remise des terrains gazzonnés ou reboisés entre les mains de leurs anciens. propriétaires, et en les replaçant ainsi dans les conditions qui en ont amené la dégradation.

$3^{\circ} \mathrm{De}$ ne pas contenir des dispositions suffisantes: pour éviter que pendant qu'on réparera les dommages d'un côté, il ne s'en produise de nouveaux d'un autre côté, par l'effet même de cette réparation.

J'avoue d'abord que je ne m'explique pás, en ce qui concerne le premier grief, pourquoi, lorsque. l'État s'associe aux travaux par des subventions, il renonce à toute répétition; tandis que lorsqu'il avance tous les frais, il ne le fait qu'à la condition de rentrer dans une partie au moins de ses déboursés. Puisque le système des subventions est appliqué, dit-on, aux travaux qui ne sont pas entièrement d'utilité publique, il semblerait que l'État devrait supporter moins 
de sacrifices pour ces travaux que pour les autres.

En outre, quand il s'agit de travaux d'urgente nécessité, ces 'travaux sont précédés d'unc enquête, tandis que pour les reboisements ou gazonnements facultalifs, ils ne le sont point; de sorte que les garanties exigées pour assurer le bon emploi des deniers de l'État, lorsque le bien public est engagé dans la question, on les juge superflues quand ces deniers sont consacrés à des dépenses d'une utilité locale, douteuse, et dans tous les cas, secondaire. Enfin, il m'est impossible de comprendre pourquoi l'administration, qui a des ressources insuffisantes pour les travaux nécessaires, en consacre une partie à des travaux dont on pourrait se passer. A cela on objecte que l'utilité des travaux facultatifs doit être en vérité consịdérée comme secondaire, si l'on envisage-les terrains à consolider dâns leur ensemble; mais qu'elle est tout aussi grande que celle des travaux dits obligatoires, pour la part du travail facultatif que représente la subvention; car la loi dit expressément que la subvention sera proportionnée à la part d'intérêt général que présente le travail à entreprendre. L'intérêt général entre-t-il pour un quart par exemple dans tel reboisement, la subvention sera d'un quart de la dépense. Tout cela peut être ingénieux, est-ce bien sérieux?

Au surplus, je repousse les primes et les subventions au nom de l'économie politique:

En thèse générale, lorsqu'une industrie n'est point engendrée par le libre jeu des intérêts indivi- 
duels, par la spontanéité et l'initiative privées, il est imprudent, hasardeux pour le moins, de l'encourager au moyen d'un appât extraordinaire, anormal, factice, et qui peut dès lors être trompeur. C'est lui donner un mobile, un fondement fort incertain, et par conséquent exposer à des mécomptes les gens qui y consacreraient leurs soins et leurs capitaux. On pourrait assurément, en y allectant les encouragements nécessaires, introduire en France la culture de l'Ananas. Serait-ce une conquête utile, désirable? non évidemment, puisqu'elle ne saurait se maintenir qu'à la condition de jouir éternellement de la subvention qui lui aurait été primitivement accordée. On ne doit recourir aux primes, aux subventions, qu'arec beaucoup de réserve et dans des cas exceptionnels. On comprend ces moyens de provoquer, de développer l'industrie au début d'une civilisation, lorsque l'intérêt privé n'a pas encore l'œil ouvert sur les ressources qu'il pourrait avantageusement exploiter; lorsqu'il manque des épargnes, des capitaux indispensables pour fonder une entreprise; lorsqu'il n'y a pas d'institutions de crédit. Au temps où nous vivons, ils ne sont plus admissibles.

S'il n'y avait jamais eu de bois dans les contrées où il s'agrit d'en établir; si l'on était dans l'ignorance ou dans le doute sur les avantages des biens-fonds de cette nature pour ceux qui les possèdent; si l'on pouvait enfin espérer qu'une fois les frais de premier établissement faits, une fois la propriété constituée, elle durerait par sa propre force à cause du profit 
qu'en retirerait le détenteur, j'admettrais les subventions et les primes; mais il $\mathrm{y}$ a eu et il y a même encore des bois dans les montagnes; on sait parfaitement que ceux-là ont disparu et que ceux-ci sont en train d'en faire autant, parce que leur conservation ne s'accorde pas avec l'intérêt actuel des propriétaires, et que dans l'échelle des cultures lucratives, la culture forestière occupe souvent le dernier rang; alors, à quoi bon imposer à l'État de grands sacrifices pour reconstruire un édifice qui serait destiné à s'écrouler infailliblement de nouveau, dès que la main du gouvernement ne serait plus là pour le soutenir?

Je ne dis rien d'exagéré : ce qui se passe aujourd'hui, l'impuissance des efforts que fait l'administration pour sauver le peu de forêts qui existent encore dans les Alpes, dans la Lozère, dans l'Ardèche, dans les Cévennes, montrent jusqu'à l'évidence que ce genre de propriété ne se concilie pas toujours avec les exigences de l'intérêt privé et qu'il n'y a ni loi, ni contrainte qui puisse en empêcher la ruine, lorsque cetle ruine n'est pas susceptible d'appauvrir le propriétaire actuel. Malgré la prohibition du défrichement, malgré la tutelle que le gouvernement exerce sur les communes, les bois des particuliers et ceux des communes elles-mêmes disparaissent rapidement; c'est un fait constaté.

Les bois des communes disparaîtront un peu moins vite peut-être, si on adopte les mesures que j'ai proposées pour. renforcer la tutelle que l'État exerce sur 
eux. Cependant on aurait tort, je l'ai dit, de compter entièrement sur ces mesures.

Mais puisque toute la force d'une grande administration ne parvient pas à sauvegarder les bois des particuliers et ceux des communes existants encore, est-il sage, est-il raisonnable de songer à en créer de nouveaux, pour les placer dans les mèmes mains et les soumettre en conséquence aux mèmes causes de destruction? Oui, sans doute, les particuliers et les communes consentiront à reboiser leurs terrains vagues si on leur donne, sous forme de primes, d'exemptions d'impôts, de subventions, des subsides qui les indemnisent à la fois et des frais du reboisement et de la perte qu'on leur occasionnera par la suppression des pâturages; mais quand les bois auront été formés, comment fera-t-on pour en assurer la conservation? L'État priendra-t-il à sa charge les frais de gestion et continuera-t-il les mêmes subsides? ce serait le seul moyen. Mieux vaudrait cent fois recourir immédiatement à l'expropriation pour cause d'utilité publique.

Ainsi, on est forcé de reconnaître que les fonds employés en subventions et en primes, non-seulement pour les particuliers, mais encore pour les communes, sont des fonds fort mal employés et que l'on peut considérer d'avance comme perdus. On comprend d'ailleur's que les travaux de consolidation des terres, de reboisement et de gazonnement, exécutés d'après ce système, n'étant assujetlis à aucun plan d'ensemble, à aucune direction, n'ayant entre 
eux aucune solidarité, ze sauraient produire que des résultats insignifiants.

Le premier reproche que j'ai fait aux lois du reboisement et du. gazonnement est donc fondé. Le deuxième ne l'est pas moins :

L'administration, elle-même, a montré qu'elle n'avait pas grande confiance dans les résultats des travaux facultatifs exécutés à l'aide de ses encouragements, puisqu'elle s'est réservé d'exécuter à ses frais et de diriger, dans tous les cas, les travaux obligatoires. Mais s'il y a lieu de craindre que les forêts créées en grande partie avec les fonds des communes et des particuliers ne soient pas entretenues en bon état, à plus forte raison doit-on s'inquiéter de l'avenir de celles à là création desquelles les communes et les particuliers n'auront pas voulu participer; et c'est un vice fondamental de la loi d'avoir admis que les terrains pourraient être remis, après le complet achèvement des travaux, entre les mains des anciens propriétaires. Rien ne justifie une semblable générosité de la part de l'État. Il est évident que les terrains que l'on consolide n'auront de valeur que par les travaux qu'on y fait; que leur prix réel actuel est dans tous les cas peu de chose à côté de la dépense qu'occasionnent lesdits travaux; pourquoi donc l'État ne les acquerrait-il pas définitivement dès à présent, et s'engage-t-il à en rétrocéder la moitié ou les trois quarts? Eh quoi! le gouvernement s'empare de ces terrains; il y dépense des sommes énormes en barrages, en semis, en plantations, sans compter 
les indemnités pour interruption de jouissance; et quand il est parvenu à les restaurer au prix de si grands sacrifices, il en abandonne de nouveau une partie à des mains qui la laisseront encore se dégrader! Vraiment, ce n'est pas raisonnable. C'est d'autant moins raisonnable que la valeur qu'auront acquise ces terrains, est surtout une valeur immatérielle, une valeur d'utilité publique, qui ne saurait s'apprécier en argent, et dont l'individu ne tient aucun compte; de sorte qu’en la cédant à des particuliers, l'État s'appauvrit sans profit pour eux. On pourrait objecter que, pour les particuliers, cette rétrocession n'est point à craindre, attendu qu'en présence de la tendance des jurys d'expropriation à exagérer la valeur des propriétés auxquelles l'État applique la loi d'expropriation pour cause d'utilité publique, il y a grande probabilité que les particuliers auront reçu pour le prix de leurs terrains, une somme plus élevée que celle qui représentera la valeur de ces terrains, après l'exécution des travaux. Cette probabilité pourrait bien cependant ne pas se réaliser, quand on considère que les terrains à restaurer sont souvent complétement improductifs et sous le coup d'une destruction complète, imminente. Quelle que. soit la libéralité des jurys d'expropriation, il n'y aurait rien d'étonnant à ce qu'ils attribuassent à ces terrains un prix moins élevé que celuj qu'ils acquerront, quand ils auront été consolidés et mis en valeur. Au surplus : ou le danger que je signale est réel et alors mon objection reste; ou il est chimérique et alors il 
n'y avait aucun motif pour que l'on rendît l'expropriation conditionnelle.

Je laisse de côté, comme on voit, l'hypothèse du remboursement des frais de restauration, par les propriétaires des terrains. Cette hypothèse est irréalisable en effet, puisque les frais de restauration seront certainement hors de toute proportion avec le revenu net que pourront rapporter les terrains, après qu'ils auront été remis en valeur. D'ailleurs, qui ne voit combien serait difficile une ventilation équitable entre les propriétaires, quand il s'agirait de faire à chacun sa part dans les dépenses de certains de ces travaux, tels que les barrages qui, utiles également à toutes les propriétés, n'auraient été cependant établis que sur quelques-unes.

Justifions maintenant le troisième reproche:

J'ai dit que c'était une lacune bien regrettable dans la loi qu'elle ne contînt pas les dispositions suffisantes pour éviter que, pendant qu'on réparerait les dégẩts d'un côté, il ne s'en produisît de nouveaux d'un autre côté, par l'effet de cette réparation.

Les bois restent donc soumis tout simplement au régime forestier, tel que l'ont organisé le code forestier et l'ordonnance royale du $1^{\text {er }}$ août 1827 , régime insuffisant; et les pâturages, non englobés dans les périmètres de conservation obligatoire, restent livrés à l'insouciance et à l'avidité de ceux qui les possèdent. Or qu'arrive-t-il ? C'est que, tandis qu'on s'efforce de reboiser les terrains dégradés, les troupeaux auxquels on enlève ainsi une partie de leur 
domaine, s'accumulent sur l'autre partie déja surchargée et en précipitentla ruine. On ne saurait être plus imprévoyant.

Voilà ce que je vois de mauvais dans les lois sur le reboisement et sur le gázonnement des montagnes ${ }^{1}$, et voici maintenant ce que $j$ 'y vois d'excellent :

D'abord elles proclament le droit d'intervention de l'État dans la gestion, non pas seulement des bois, mais encore des pâturages et des terrains incultes appartenant aux communes;

Ensuite elles reconnaissent que c'est un devoir pour le gouvernement de venir au secours des communes, quand celles-ci n'ont pas les ressources qu'exigerait l'amélioration de leurs biens.

Enfin, elles àdmettent l'expropriation pour cause d'utilité publique des propriétés de même nature que les particuliers seraient inhabiles à régénérer ou à conserver; et on peut dire qu'elles admettent aussi cette expropriation pour les terrains communaux; car la faculté qu'elles donnent à l'État de retenir la moitié ou le quart de ces terrains, après l'exécution des travaux, aboutit à une expropriation partielle et déguisée.

1. Je n'ai pas parlé dans ma critique de l'article $\mathbf{1 4}$ de la loi sur le reboisement, relatif à l'aliénation d'une certaine étendue de forêts, dans le but de faire face aux frais du reboisement. Cette conception singulière, qui attachait la même importance aux forêts en projet et d'une création fort incertaine, qu'aux forèts séculaires en plein rapport, a été abandonné lors de la discussion de la loi du gazonnement. Voilà pourquoi je n'en ai rien dit. 
Ces dispositions sont très-remarquables; et comme l'appréciation des conditions nécessaires pour que la conservation d'un terrain soit déclarée d'utilité publique, est abandonnée, sans restriction formelle, à l'autorité supérieure, celle-ci pourrait, avec un peu de hardiesse, rattacher au domaine de-l'État la moitié ou le quart des propriétés coinmunales et toutes les propriétés privées situées en montagne.

Cependant, il faut bien l'avoucr, ces lois ont été faites pour arrêter les racages des eaux et conjuier les effets des grands désordres de la nature ${ }^{1}$. Elles ne devaient pas s'appliquer, dans l'esprit du législateur, aux terrains qui, bien que situés en pente et impropres à l'agriculture, ne courent pas le risque d'être emportés dans les plaines et d'y causer des dommages. Ces terrains-là auraient pourtant besoin aussi d'être mis entre les mains de l'État; au moins, d'être surveillćs par lui, quand ils appartiennent aux communes, et en cela encore mes idées vont plus loin que les lois sur le reboisement et le gazonnement.

J'avais néanmoins raison de dire que ces lois renfermaient des dispositions qui étaient d'accord avec mon opinion sur l'autorité dont le gouvernement devrait être investi relativement aux forêts et pâturages créés ou à créer dans notre pays, opinion que je résume ainsi :

$1^{\circ}$ Instituer pour les forêts, les bois et les pâtu-

1. Rapport à l'Émpereur, page 8. 
rages un régine spécial qui serait intitulé : régime forestier et pastoral et dont l'application serait confiée à l'administration des forêts qui deviendrait l'administration des forêts et des pâturages;

$2^{\circ}$ Mettre entièrement entre les mains de l'État, pour être gérés conformément à ce régime, les terrains dont la conservation serait d'intérêt général et ne pourrait être assurée que par le boisement ou le gazonnement.

L'administration des forêts serait investie, pour. constituer et gérer ce domaine, de toutes les prérogatives qu'accorde à l'État la loi sur l'expropriation pour cause d'utilité publique; mais son autorité serait modifiée, en ce qui concerne la délimitation, d'une manière conforme au droit commun; et les dispositions du code forestier relatives au cantonnement des droits d'usage seraient abrogées, pour être remplacées par d'autres dispositions qui ne tendraient qu'à régulariser la jouissance de ces droits et à l'empêcher' d'empiéter sur celle du propriétaịre;

$3^{\circ}$ Maintenir les bois des particuliers qui n'auraieñt pas été acquis par l'État en exécution de l'apticle précédent, sous l'empire de la prohibition, toutes les fois que leur conservation, sans être d'intérêt général, se rattacherait à des intérêts locaux d'une réelle importance, et faire de ces bois l'objet d'un cadastre spécial; mais pour dédommager les propriétaires de la restriction apportée à leur jouissance, leur accorder une indemnité sous la forme que j'ai indiquée page $/ 451$ (suppression des droits 
d'octroi, diminution de l'impôt foncier, association de l'administration publique à la poursuite et à la répression des délits, subventions pour, la construction de chemins de vidange);

$4^{\circ}$ Soumettre les forêts et les pâturages et tous les terrains à boiser'ou à gazonnér des communes et des établissements publics qui ne seraient pas compris dans le domaine prévu par l'article 2, ou qui n'y auraient pas encore été rattachés, à la tutelle administrative modifiée en ce sens :

Qu'elle donnerait à l'administration sur les bois non susceptibles d'exploitation régulière, et sur les pâturages, tous leś droits qu'elle a déjà sur les bois susceptibles d'exploitation régulière; qu'elle lui laisserait en outre la plus grande latitude pour règler la jouissance de ces biens de la manière la plus favorable aux intérêts des propriétaires, et qu'elle lui permettrait ên conséquence d'y áutoriser même des cultures temporaires.

Qu'elle lui conférerait toute l'autorité voulue pour assurer : $1^{\circ}$ la gestion ordinaire dans laquelle seraient comprises, indépendamment des opérations prévies par l'article 107 du code forestier, la surveillance, la constatation des délits et l'étude des aménagements; $2^{\circ}$ l'entretien, les assainissements, les délimitations, l'exécution de tous les travatix autorisés par des décisions régulières. L'administration forestière nommerait donc leś gardés; elle fixerait leur salaire; les fonds nécessaires pour assurer ce sálaire seraient, ainsi que les autres dépenses relatives á la régie, à 
l'entretien et aux travaux indispensables, inscrits au budget des dépenses obligatoires des municipalités;

Que l'État ferait l'avance desdites dépenses, sauf à se rembourser sur le prix des coupes à défaut d'autres moyens;

Que l'État, sauf à se rembourser de la même manière, pourrait en outre prêter de l'argent aux communes, quand elles en auraient besoin pour les grands travaux neufs d'amélioration tels que les routes, les reboisements d'une certaine étendue;

Que la répétition de l'État se bornerait toutefois pour les frais de la régie, définie comme elle l'est plus haut, au chiffre à établir, exprimant le rapport entre les frais de régie et les produits principaux des forêts les plus productives.

Énergique intervention de l'État: telle est en définitive la nécéssité qui s'impose, quand on étudie. les moyens d'améliorer les parties de notre territoire qui ne peuvent être cultivées qu'en prés ou en bois.

Celte nécessité sera contredite par ceux de mes concitoyens, et ils sont nombreux, qui, malgré la divergence de leurs opinions sur presque toutes les questions politiques, se trouvent d'accord lorsqu'il s'agit de porter atteinte aux prérogatives de l'État.

Mais que ces messieurs me permettent de le leur dire. : parmi tous les dangers qui -menacent encore notre malheureuse patrie déjà si éprouvée, je n'en vois pas de plus grand que. celui auquel l'exposent leurs théories gouvernementales. Si la France entrait 
dans la voie où ils veulent l'engager avec les meilleures intentions du monde, je n'en dóute pas, son heure aurait sonné : il ne resterait bientôt d'elle que quelques individualités plus ou moins remarquables; comme corps de nation, elle descendrait au dernier rang, elle n'aurait plus de mission à remplir. J'espère donc qu'elle finira par voir le néant des chimériques espérances que les décentralisateurs font briller à ses yeux, et qu'après avoir été la première à proclamer dans le monde, non plus seulement au nom de la charité chrétienne, mais au nom de la raison et de la science, le principe de la solidarité, elle ne voudra pas se démentir. Il y va de sa gloire, de sa sécurité, et de l'avenir même de la civilisation.

Les hommes sont unis par certains intérêts qui leur sont communs, et qui ne sauraient être sauvegardés que par une autorité assez puissante pour .vaincre toutes les résistances. Cette autorité, l'État seul peut l'avoir, et, puisque la conservation des bois et dés pâturages est au nombre de ces intérêts; puisque d'ailleurs elle entraîne - des exigences auxquelles l'initiative individuelle ne saurait satisfaire, il n'y a que l'État qui soit capable de l'assurer.

Je sais bien que dans ces derniers temps,'il s'est élevé des protestations au sujet de l'utilité que l'on avait jusqu'alors, d'une voix unanime, attribuée aux forêts. Ces protestations viennent d'un malentendu : ainsi, nous avons vu des savants s'efforcer d'établir que les forêts n'ont pas d'influence appréciable sur les grands phénomènes cosmologiques, et nous en 
avons vu d'autres conclure de là que l'État n'avait pas plus à s'en préoccuper qu'il ne sé préoccupe des autres cultures. 'Or, est-il nécessaire de le faire remarquer, en posant le problème de cette manière, on l'a fort mal posé.

Que les forêts n'aient aucune influence.sur la masse d'eau que le soleil pompe chaque année dans les mers, pour la reverser sur les continents; qu'elles n'en aient pas davantage sur la distribution générale de la chaleur à la surface du globe et sur les courants qui traversent l'atmosphère, c'est possible; mais qu'est-ce que cela prouve? Est-il vrai que les forêts peuvent modifier les circonstances particulières par lesquelles ces grands phénomènes se manifestent aux hommes? Voilà la question, et personne ne conteste qu'elle ne doive être résolue par l'ạffirmative.

Il importe peu, au point de vue des conditions de la vie humaine, que. la température moyenne annuelle d'une région ne change pas, si les variations de cette température sur un point donné, deviennent telles, que ce point soit rendu inhabitable. De même, il est indifférent que la quantité d'eau qui tombe chaque année sur une partie du globe reste la même, si la distribution et l'écoulement de cette eau éprouvent des perturbations. Ce. ne sont pas les moyennes, ce sont les chiffres sur lesquels reposent ces moyennes, qui touchent les hommes; ce ne sont pas les phénomènes généraux, ce sont les phénomènes locaux; ce n'est pas l'en- 
sernble des choses, ce sont les détails, car l'humanité elle-même n'est qu'un détail dans'la vie universelle, C'est un détail aussi que quatre - de nos départements se dépeuplent, et que leurs habitants aillent en Amérique continuer dans les montagnes Rocheuses, ces défrichements insensés qui ont stérilisé leur sol natal ; mais pour la France et même pour l'humanité tout entière, ce détail est un événement lamentable.

FIN. 


\section{TABLE DES MATIÈRES.}

Pages.

Préface . . . . . . . . . . . . . . . . I

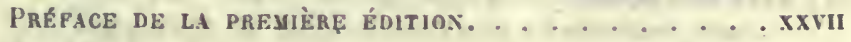

Introduction ..................... 1

\section{PREMIERE F́TUDE.}

DE LA STATISTIQCE.

CHAPITRE PREMIER. - De la statistique en général, et des études qu'elle comporte au point de vue de l'aménagement. . . . . . . . . . . . . 17

CHAP. II. - Renseignements généraux. . . . . . 20̈

Article preiter. Plan de la forèt. . . . . . . 20

Art. II. Etat de la forêt considérée dans les éléments qui la constituent ou qu'elle renferme. . . . . . 26

ART. II. Conservation et entretien. . . . . . . 38

Аrт. Iv. Dépenses. . . . . ......... . . 39

ART. v. Exploitation et produits ........ 40

Art. vi. Débouchés . . . . . . . . . . 42 42

CHAP. III. - Renseignements spéciaux ....... . 4 .

Article premier. Points à examiner. . . . . . 44

Art. I1. Du parcellaire. . . . . . . . . 40

Art. 11. Description spéciale. . . . . . . . 53

Art. IV. Résumé . . . . . . . . . . . . อ̃ 
Art. v. Observations sur les principes ci-dessus développés .............. s̈t

AnT. vi. Du nombre et de la forme des pièces relatives au parcellaire et à la description spéciale. . . . . $\quad 59$ Plan et description d'une parcelle, etc. . . . . . 61

\section{DEUXIËUE FUTUE.}

\section{DE L'EXPL OITABILITÉ:}

But et division de celle élude. . . . . . . . .

CHAPITRE PREMIER. - De l'exploitabilité, ảbstraction faite des exigences de la végétation et de la culture . .

Article premer. De l'exploitabilité relative aux produits matériels les plus considérables, ou de l'exploi-

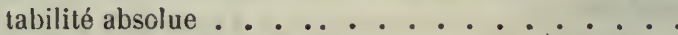

$\$$ fer. Lois générales sur lesquelles repose la recherche de l'exploitabilité absolue.......

§2. Des moyens par lesquels on détérmine l'àge qui correspond à l'exploitabilité absolue . . ... . 73

§3. Utilité pratique de l'exploitabilité absolue. . . 79

Arт. II. De l'exploitabilité relativé aux produits les plus utiles. . . . . . . . . . .

ArT. III. De l'exploitabilité relative au plus grand produit en argent. . . . . . . . .

Anr. Iv. De l'exploitabilité relative au revenu le plus élevé par rapport au capital . . . . . . . . $\$$ fer. Considérations générales sur la valeur et le profit des capitaux et spécialement des fonds de

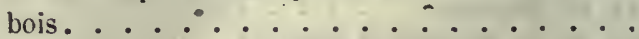

$\$$ 2. De l'exploitabilité relative à la rente la plus élevée, dans son application à un arbre isolé. . . .

§3. De l'exploitabilité relative à la rente la plus élevée, dans śon application à un massif non aménagé..............

$\$$ 4. De l'exploitabilité relative à la rente la plus élevée, dans son application à un massif aménagé. . 
Ð . Lois auxquelles est soumise l'exploitabilité reldtive à la rente la plus élevée . . . . . .

Pages.

CHAP. II. - De l'exploitabilité dans ses rapports avec les exigences de la végétation et de la culture. . . 126 Article premier. Choix du mode d'exploitation ...

$\$ \mathcal{A e r}^{\mathrm{e}}$. Du taillis simple et de la futaie au point de vue cultural ............

\$2. Du taillis simple et de la fulaie au point de vue économique. . . . . . . . 134

\$ 3. Conclusion des deux paragraphes précédents. . 133̈

- 4. Des réserves dans les taillis . . . . . 136

Ant. 11. De l'exploitabilité dans les taillis simples . . 139

Art. III. De l'exploitabilité dans les futaies. . . . 142

ART. Iv. De l'exploitabilité dans les taillis composés . . 148

ART. v. Des mesures à prendre pour assurer la détermination de l'exploitabilité. . . . . . . 149

CHAP. III. - De l'exploitabilité dans ses rapports avec l'iutérèt du propriétaire. . . . . . . 152

Article premier. De l'exploitabilité dans ses rapports avec l'intérêt de l'État. . . . . . . 153

ARr. II. De l'exploitabilité dans ses rapports avec l'intéret des particuliers. . . . . . . . 157

ART. III. De l'exploitabilité dans ses rapports avec l'intérèt des communes. . . . . . . . 162

CHAP. IV. - De l'exploitabilité dans ses rapports avec l'intérèt public. . . . . . . . . . 16z

\section{TRÖISIÈME ÉTUDE. -}

DU PLAN D'EXPLOITATION.

CHAPITRE PREMIER. - Principes fondamentaux . . .

CHAP. II. - Du plan d'exploitation dans les taillis sim-

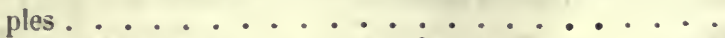
Article premier. Du tableau des expluitations et des difficultés que rencontre sa formation . . . . 480 
$\Lambda$ RT. II. Classement des parcelles suivant l'àge d'exploitabilité ...............

ART, III. Class.ıment des parcelles conformément aux règles d'assiette des coupes. .........

ART. Iv. Classement des parcelles conformément aux exigences du rapport annuel soutenu ......

$\$ 1 \mathrm{er}$. Conditions nécessaires pour que le rapport annuel soutenu puisse être réalisé. . . . . . .

§. Du rapport soutenu dans l'hypothèse d'une révolution prinćipale définitive et d'une marche des coupes normale ...........

1. Des moyens de remédier à l'inégalité des conditions de la végétation . . . . . . .

2. Des limites dans lesquelles il parait convenable de renfermer l'emploi des coefficients de

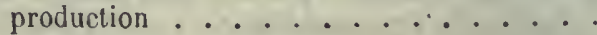

§ 3. - Du rapport soutenu dans l'hypothèse d'une révolution principale définitive et d'une marche des coupes provisoire . . . . . . .

$\$$ 4. Du rapport soutenu dans l'hypothèse d'une révolution principale transitoire et d'une marche des coupes provisoire . . . . . . . .

Art. v. Résumé et conclusion. . . . . . . .

CHAP. III. - Du plan d'exploitation dans les futaies traitées par la métlode du réensemencemént naturel et des éclaircies périodiques. . . . . . . . .

Article premier. Des raisons culturales qui s'opposent à ce que l'on adopte, pour le tableau des exploitations des futaies, le même cadre que pour celui des

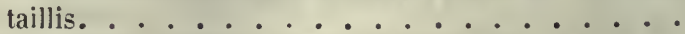

ART. II. De la division de la révolution en périodes et du partage de la forêt en affectations correspondantes.

CHAP. IV. - Du plan d'exploitation dans les futaies, la révolution pouvant être définitive et la marcle des coupes normale. . . . . . . . . . 243 Article premier. Formation des affectations. . . . 243

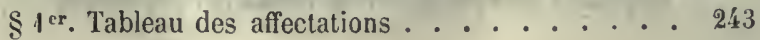


§. Formatión des affectations suivant l'âge d'exploitabilité............. 246

\$3. Formation des affectations conformément aux règles d'assiette. .............

\$. Formation des affectations conformément aux exigences du rapport soutenu. . ......

1. Des motifs qui doivent faire adopter en général, pour les affectations, des contenances égales .

2. Des motifs qui peuvent faire adopter, pour les affectations, des contenances réduites . . . 269

ART. II. Règlement des exploitations par période . . . 270

Art. III. Règlement spécial des exploitations annucles.

CHAP. V. - Du plan d'exploitation dans les futaies, la révolution pouvant êtro définitive et la marche des coupes devant ètre provisoire . . . . . . . . 303

- Article prearer. Formation des affectations. . . .

$\S$ Ier. Utilité de la formation immédiate des affectations normales ...........

$\$ 2$. Des facilités que donne, pour la.formation des affectations, l'adoption d'une marche provisoire pour les coupes. . . . . . . . 30s

Art. II. Règlement des exploitations par période . . 309

$\S$ 1 er. Coupes principales ou de régénération . . . 309

$\S$ 2. Des modifications que le rapport soutenu pourrait faire apporter au tableau des coupes principales. ............. 343

\$. Coupes d'amélioration ....... 316

ART. ıIr. Règlement spécial des exploitations annuelles.

CHAP. VI. - Du plan d'exploitation dans les futaies, la révolution devant être principale et transitoire ou simplement préparatoire, et ła marche des coupes provisoire .

Article premier. Utilité de la formation préalable du tableau des affectations normales........ 303 303

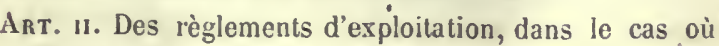
l'àge trop avancé des massifs les plus jeunes ne per- 
met pas de les laisser sur pied jusqua à la fin de la révolution norınale.......... 328

ART. III. Des règlements d'exploitation, dans le cas où l'àge trop peu avancé des massifs les płus vieux ne permet pas dē les régénérer immédiatement . . . .

CHגP. VII. - Conversions des taillis composés en futaies pleines. . . . . . . . . . . 334

Article premier. Observations préliminaires. . . . 334

ART. 11. Utilité de la formation préalable des affections normales ................ 340

ART. Il. Règlement général des exploitations. . . . 3 1'2

$\S 1^{\mathrm{er}}$. Règlement général des exploitations d'un taillis composé, régulier et complet à convertir, lorsque la révolution est préparatoire et la con.. version immédiate. . . . . . . . .

$\S$ 2. Règlement général des exploitations d'un taillis composé complet et régulier à convertir, lorsqu'il n'y a pas de révolution préparatoire et que la conversion est immédiate .........

§ 3. Règlement général des exploitations d'ùn taillis complet et régulier à convertir, lorsqu'il n'y a pas de révolution préparatoire et que la conversion est graduelle. . . . . . . . 330

I. Exposé-des méthodes applicables . . . . 330

II. Observations sur les méthodes de conversion exposées dans l'article précédent . . . . .

§ 4. Règlement général des exploitations d'un taillis composé régulier et complet à convertir, lorsqu'on adopte-une révolution préparatoire et que la conversion est graduelle. .......

§ b. Règlement général des exploitations lorsque les taillis à convertir sont irréguliers et-incomplets. 371

ART. IV. Règlement spécial des exploitations pendant la première période, quelle que soit la méthode adoptée pour la transformation. . . . . . . . 


\section{QUATRIÈME ÉTUDE.}

DE LA DIVISION D'CXE FORÉT EN MASSES, EN SECTIONS, EX Séries, des avéliorations, de la rédaction de projet D'A YYEXAGEYLNT, DE L'APPLICATION ET DC CONTROLE.

CHAPITRE PREMIER, - Des masses, des sections, des séries . . . . . . . . . . 383

Article previer. Observations préliminaires. . . . 383

ART. 11. Circonstances qui sont contraires à la division d'une forèt en séries. ........... $\$ f^{e r}$. Impossibilité de former des' séries compactes ou indépendantes l'une de l'autre . . . . . 38د

S 2. Étendue trop petite de la forêt. . . . . . 386

$\S 3$. Exigence de l'assiette et de la vidange des coupes.............. 387

ART. III. Circonstances qui reudent avantageuse ou nécessaire la division d'une forèt en séries. . . . . 388

$\S f^{\text {er }}$. Diversité des modes d'exploitation . . . 388

\$2. Diversité des ḋges d'exploitabilitẻ . . . . 389

$\S$ 3. Morcellement des classes d'áge . . . . . . 389

$\$$ 4. Exigences du rapport soutenu. . . . . . 390

$\S$ s. Application, contròle et rectification de l'aménagement. . . . . . . . . 392

$\$$ 6. Diminution des frais de transport des boiz . . 393

$\$ 7$. Egalisation du prix de revient des bois aux lieux de consommation. ........

$\$ 8$. Besoins de la consommation en bois de diverses espèces . . . . . . . . 393

§ 9. Droits d'usage. . . . . . . . . 395

Ant. 1v. Tabléau des séries. . . . . . 396

CHAP. II. - Des améliorations . . . . . . . 398 Article Premer. Observations préliminaires. . . . . 398 Arт. 11. Améliorations relatives au sol. . . . . 400 ART 111. Améliorations relatives au peuplement. . . \$01 Ant. Iv. Améliorations relatives aux voies de vidange. . $\$ 03$ Ant. v. Améliorations relatives à la surveillance et à l'entretien. ............ \$08 
Ant. vı. État collectif des améliorations . . . . 410

CHAP. III. - Rédaction du projet d'aménagement, application et contrôle . . . . . . . . . .

Article premier. De la rédaction du projet d'aménage-

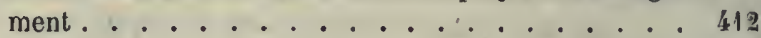
ArT. 11. Application et contròle de l'aménagement. . . 416 GINQUIÈME ÉTUDE.

ÉTAT NORMAL DES FORẼTS ENVISAGÉE DANS LEUR ENSEMBLE. TRAVAUX A raIRE POUR LE RÉTABLIR EX FRANCE. LOIS ET RÉLEMENTS FORESTIERS. ATTRIBUTIONS DE L'ADMINISTRATION FORESTIÉRE.

CHAPJTRE PREMIER. - Ĺtat normal des forêts envisagées dans leur ensemble. Travaux à faire pour le retablir . .

CHAP. II. - Lois et règlements forestiers. Attributions de l'administration forestière. . . . . . . . Article premier. Altributions de l'administration forestière dans leurs rapports avec les biens de l'État. Arr. I1. Attributions de l'administration forestière dans leurs rapports avec les biens des particuliers. . . ART. III. Attributions de l'administration forestière dans leurs rapports avec les biens des communes. ... Art. 1v. Conclusions appuyées sur les lois relatives au reboisement et au gazonnement des niontagnes. . .

FIN DE LA TARLE.

\section{ERRATA}

Page .1, note, ligne 2, au lieu de : le cours de culture, lisez : le cours de l'École.

- $\boldsymbol{7}$, lignes 1 et 2 , au lieu de : et ne seraien pas, lisez : et que ces frais ne seraient pas.

PARIS, - J, CLAYE, IMPRIMEUR, T, RUE SAINT-BENOIT. - $|H 14|$ 





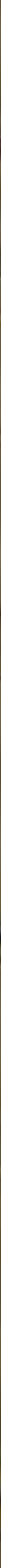




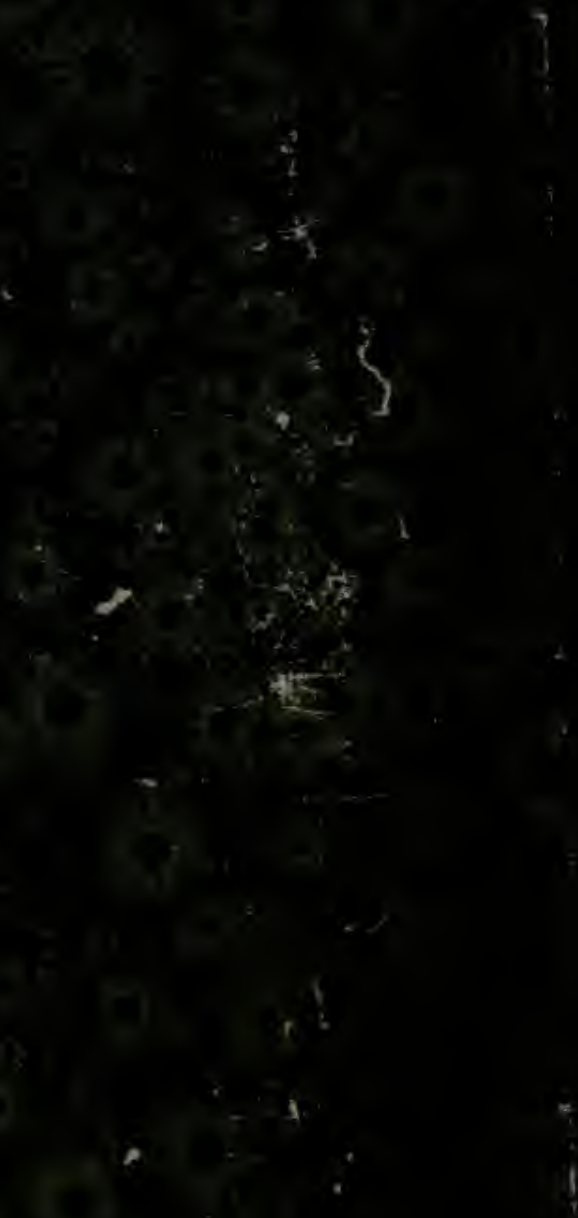